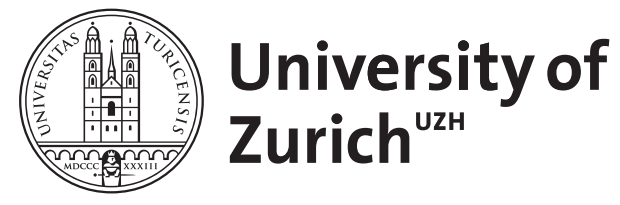

Zurich Open Repository and Archive

University of Zurich

University Library

Strickhofstrasse 39

CH-8057 Zurich

www.zora.uzh.ch

Year: 2020

\title{
Unvergleichbarkeit. Dürfen wir Entscheidungen dem Würfel überlassen?
}

Messerli, Michael

Posted at the Zurich Open Repository and Archive, University of Zurich

ZORA URL: https://doi.org/10.5167/uzh-187478

Monograph

Published Version

Originally published at:

Messerli, Michael (2020). Unvergleichbarkeit. Dürfen wir Entscheidungen dem Würfel überlassen? Basel: Colmena. 


\section{Michael Messerli}

Unvergleich-

barkeit.

Dürfen wir

Entscheidungen

dem Würfel

überlassen?

Colmena 


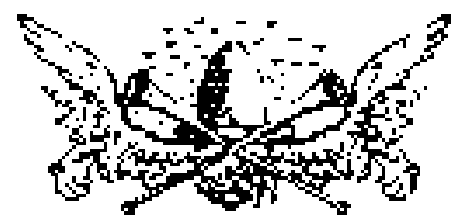




\section{Colmena Philosophica IV}

Herausgegeben von

Wolfgang Rother

Wissenschaftlicher Beirat

Christine Abbt (Luzern)

Emil Angehrn (Basel)

Martin Bondeli (Bern)

Michael Erler (Würzburg)

Gerald Hartung (Wuppertal)

Marion Heinz (Siegen)

Ursula Renz (Klagenfurt)

Peter Schaber (Zürich)

Andreas Urs Sommer (Freiburg i. Br.)

Giovanni Ventimiglia (Luzern)

Simone Zurbuchen (Lausanne) 

Michael Messerli

Unvergleich-

barkeit.

Dürfen wir

Entscheidungen

dem Würfel

überlassen?

Colmena 
Publiziert mit Unterstützung des Schweizerischen Nationalfonds zur Föderung der wissenschaftlichen Forschung

\section{(c) $(i)$ \\ BY NC ND}

(C) 2020 Colmena Verlag, Basel

Print: ISBN 978-3-906896-11-3

E-Book (PDF): DOI 10.46301/colmena.96113 
Inhalt

Vorwort

Kapitel 1: Inkommensurabilität und Unvergleichbarkeit

1. Die Begriffe der Inkommensurabilität und Unvergleichbarkeit 22

2. Weshalb keine Begriffsanalyse?

3. Begriffsexplikation, Stipulation und reale Elemente von Unvergleichbarkeit

4. Zusammenfassung

Kapitel 2: Eine Präferenzversion von Unvergleichbarkeit

1. Welcher Begriff der Präferenz ist im Zusammenhang mit Unvergleichbarkeit relevant?

2. Präferenzen, Gründe und praktisches Nachdenken

3. Mögliche Einwände und Abgrenzung $\quad 62$

4. Zusammenfassung

Kapitel 3: Verschiedene Typen: Evaluatives Unwissen als wichtigstes Argument für Unvergleichbarkeit

1. Drei benachbarte Phänomene: Abgrenzung 73

2. Verschiedene Typen harter Fälle $\quad 78$

3. Zusammenfassung 93

Kapitel 4: Argumente gegen Unvergleichbarkeit: Zurückweisung 95

1. Einwände 96

2. Weitere Argumente für Unvergleichbarkeit 102

3. Zusammenfassung 105

Kapitel 5: Unvergleichbarkeit, (In)Transitivitätund Gleichgültigkeit 107

1. Das Argument der kleinen Verbesserung 109

2. Die Rolle der (In)Transitivität im Argument der kleinen Verbesserung 112

3. Was kann hinsichtlich Unvergleichbarkeit gefolgert werden? 117

$\begin{array}{ll}\text { 4. Zusammenfassung } & 125\end{array}$

Kapitel 6: Unvergleichbarkeit und Rationalität $\quad 127$

1. Diskussion von Changs Position: Vergleichbarkeit ist notwendig für Rationalität $\quad 129$

2. Unvergleichbarkeit und Rationalität: verschiedene Ansätze 132 
3. Unvergleichbarkeit und Rationalität: Welche Sichtweise ist relevant? 138

4. Vergleichbarkeit, Begründung und das Problem harter Fälle 145

5. Zusammenfassung 152

Kapitel 7: Die Trichotomie-These und vage Entscheidungskriterien $\quad 155$

1. Die Trichotomie-These 157

2. Changs Argumentation gegen die Trichotomie-These 160

3. Das Ketten-Argument 160

4. Bisherige Ansätze 163

5. $\quad$ Ein Gegenbeispiel 168

$\begin{array}{ll}\text { 6. Mögliche Kritik } & 170\end{array}$

7. Vage Entscheidungskriterien 171

8. Zusammenfassung 176

Kapitel 8: Parität $\quad 177$

1. Changs Ausführungen zu Parität 179

2. Gerts Interpretation von Parität 180

3. Können harte Fälle mithilfe von Parität aufgelöst werden? 186

4. Zusammenfassung 188

Kapitel 9: Klumpentheorie $\quad 191$

1. Die Theorie 193

2. Diskussion 200

3. Können harte Fälle mit der Klumpentheorie aufgelöst werden? $\quad 207$

$\begin{array}{ll}\text { 4. Zusammenfassung } & 214\end{array}$

Kapitel 10: Weshalb nicht beliebig wählen? $\quad 215$

1. Beliebig wählen 218

2. Gründe für das beliebige Wählen 222

3. Bedingungen für das beliebige Wählen 226

4. Gründe gegen das beliebige Wählen 233

5. Zusammenfassung 243

\begin{tabular}{ll} 
Rückblick & 245 \\
\hline
\end{tabular}

\begin{tabular}{ll}
\hline Literaturverzeichnis & 247
\end{tabular}

Personenregister $\quad 257$

$\begin{array}{ll}\text { Sachregister } & 259\end{array}$

Zu Autor und Buch $\quad 260$ 


\section{Vorwort}

Beim vorliegenden Buch handelt es sich um die überarbeitete Version meiner Dissertation, die 2017 an der Ludwig-MaximiliansUniversität München verteidigt wurde. Monika Betzler hat mich im Studium, beim Antrag für die Förderung durch den Schweizerischen Nationalfonds und bei der Dissertation sehr unterstützt. Ihre Verbesserungsvorschläge und kritischen Anmerkungen haben die Doktorarbeit und das daraus entstandene Buch stark beeinflusst. Dafür bin ich ihr zu grossem Dank verpflichtet. Ebenso danke ich Stephan Sellmaier, dass er sich bereit erklärt hat, die Arbeit mitzubetreuen, als sich diese bereits in einem fortgeschrittenen Stadium befand. Seine Kommentare und Anregungen halfen mir, insbesondere Kapitel 5 und einen Artikel zu verbessern.

Ein spezieller Dank gilt Verena Messerli und Jonas Pfister, die das gesamte Manuskript mit grosser Sorgfalt gegengelesen haben. Ihre inhaltlichen und sprachlichen Korrekturen waren sehr wertvoll.

Die Zusammenarbeit mit Kevin Reuter ist immer wieder inspirierend und eine Freude, wofür ich ausserordentlich dankbar bin.

Allen, die mich in der Doktorarbeit unterstützten, danke ich bestens, inbesondere Dominik Aeschbacher, Kathrin von Allmen, Claus Beisbart (dem ich wichtige Verbesserungsvorschläge zu Kapitel 10 zu verdanken habe), Jonas Blatter, Fabio Briante, Georg Brun, Christian Budnik, Ruth Chang, Annabel Colas, Sebastian Elliker, Fabrizio Fasciati, Magdalena Hoffmann, Martin Hurni, Silvan Imhof, Jörg Löschke, Simon Messerli (wertvoll seine Hinweise vor allem zu Kapitel 3), Peter Messerli, Tim Räz, Tanja Rechnitzer, Hans Rott, Peter Schaber, Nina Scherrer, Lara Tischler und Jan Walker.

Grosser Dank gilt auch meiner Lektorin Marianne Wackernagel vom Colmena Verlag. Dank ihrer zahlreichen Verbesserungen und Korrekturen konnte die Qualität des Buches nochmals gesteigert werden.

Und nicht zuletzt möchte ich dem Schweizerischen Nationalfonds (SNF) danken, der sowohl die Dissertation als auch die OpenAccess-Buchpublikation finanzierte. 

Einleitung 
Es ist ein wichtiges Gut, selbst entscheiden zu können, was wir tun und wie wir leben wollen. Investitionen in den Fortschritt der Technik lassen sich oft als das Bemühen rekonstruieren, die menschliche Entscheidungsmacht auf Lebensbereiche auszudehnen, die bis anhin der 'Lotterie der Natur' überlassen waren. Und der Kampf um individuelle Autonomie und politische Mitbestimmung bringt den Wunsch zum Ausdruck, in die eigene Hand zu nehmen, was zuvor durch andere Menschen beschlossen und verfügt wurde. Auch wenn es zu den Bedingungen menschlicher Existenz gehört, dass vieles durch die Umstände und manches durch andere entschieden wird: Die Menschen würden gerne ihre Entscheidungsmacht ausweiten. Dabei geht es uns jedoch nicht nur darum, in wesentlichen Belangen unseres Lebens selbst entscheiden zu können. Unser Interesse ist, die richtigen Entscheidungen zu fällen, das heisst, zwischen den uns offenstehenden Optionen eine rationale Wahl zu treffen. Das jedoch ist nicht immer ganz einfach. Wer die Wahl hat, hat bekanntlich die Qual. Dafür lassen sich unterschiedliche Erklärungen anführen.

Dieses Buch nimmt eine philosophische Erklärung genauer in den Blick. Es ist die Behauptung, dass rationale Entscheidungen manchmal nicht nur schwierig, sondern prinzipiell unmöglich sind. Dies ist genau dann der Fall, wenn die Optionen, zwischen denen wir wählen können, unvergleichbar sind - das heisst, wenn zwischen den Handlungsoptionen keine der Vergleichsrelationen ist besser als, ist schlechter als oder ist gleich gut wie besteht (vgl. z. B. SinnottArmstrong 1988, Schaber 1994, Anderson 1997, Rabinowicz 2009, Gustafsson und Espinoza 2010, Carlson 2011 und Constaninescu 2012). Diese Konzeption von (Un)Vergleichbarkeit wird in der Unvergleichbarkeits- beziehungsweise Inkommensurabilitätsdebatte als Trichotomie-These bezeichnet (Chang 1997). ${ }^{1}$ Sie liegt unserer traditionellen Auffassung von Vergleichen zugrunde und findet sich auch in den Rationalitätstheorien der Sozialwissenschaften. Diese sind axiomatisch so aufgebaut, dass Handlungsoptionen in einer spezifischen Ordnung stehen, genauer, dass sie sich in eine Rangordnung bringen

1 Damit ist keine reine Begriffsfestlegung, sondern eine substanzielle These gemeint (vgl. Kapitel 7). 
lassen (siehe z. B. Resnik 1990 und Mas-Colell et al. 1995). Die diesen Theorien zugrunde liegende Trichotomie-These lautet wie folgt: Zwei Optionen, $x$ und $y$, sind genau dann vergleichbar, wenn $x$ entweder besser oder schlechter als $y$ oder $x$ und $y$ gleich gut sind.

Vor diesem Hintergrund der Trichotomie-These wird deutlich, warum in Situationen, die man in der Inkommensurabilitätsdebatte harte Fälle nennt (Chang 2002a), kein Vergleich möglich ist. Harte Fälle wie beispielsweise die Berufswahl verlangen uns in der Regel Entscheidungen ab, die weitreichende Konsequenzen mit sich bringen. Zugleich scheinen die zur Wahl stehenden wertvollen Eigenschaften in keine durch die Trichotomie-These erfassbare Relation gebracht werden zu können. Wer etwa vor der Wahl steht, Anwältin oder Klarinettistin zu werden, hat gegebenenfalls grosse Schwierigkeiten, einen Beruf als besser oder schlechter als oder als gleich gut wie den anderen auszuweisen. Deshalb scheint eine rationale Entscheidung unmöglich (dafür argumentiert etwa Boot 2007).

Weil eine rationale Entscheidung, so die These, eine Abwägung der Optionen erfordert (siehe z. B. Chang 2015a) und damit deren Vergleichbarkeit im Sinne der Trichotomie-These voraussetzt, sind wir in harten Fällen zur Irrationalität verurteilt - und eben dies ist das 'Harte' an solchen Situationen. Sie stellen genuine Konflikte dar, die nicht rational lösbar sind. Einerseits kann es hierbei um ganz unterschiedliche Sachverhalte gehen: um die Berufswahl, die Wahl zwischen Wohnorten, um die Frage, ob man Kinder haben möchte oder sich lieber ganz einem öffentlichen Amt widmet, aber auch um alltägliche Entscheidungen zwischen verschiedenen komplexen Zielen, wie etwa zwischen zwei unterschiedlichen Hobbys. Andererseits kann das Vorliegen eines solchen Falles aber nicht am blossen Inhalt der Beispiele festgemacht werden. Inwiefern ein harter Fall vorliegt, hat stets auch mit der Akteurin zu tun. Dementsprechend lässt sich beispielsweise nicht sagen, dass die Berufswahl generell einen harten Fall darstellt und die Wahl, in welchem Land man leben möchte, nicht. Für einige ist bereits als Kind klar, dass sie später Pilot werden wollen, und andere haben im Sinn, nach Amerika auszuwandern. Aber für Dritte wiederum stellen diese Entscheidungen unter Umständen harte Fälle dar - in dem Sinne, dass es (i) keine beste Option gibt und (ii) dieses Fehlen einer besten Alternative nicht als Indifferenz gedeutet werden 
kann. Die Frage, wie das Konzept der Unvergleichbarkeit dabei genau vom Konzept der Indifferenz abgegrenzt werden kann, ist komplizierter, als man meinen könnte. Vereinfachend können wir an dieser Stelle aber sagen, dass es uns bei unvergleichbaren im Gegensatz zu gleichwertigen Optionen wie der Wahl zwischen identischen Heuhaufen nicht egal ist, was wir wählen (vgl. Buridans Esel).

Wenn wir in harten Fällen respektive bei einer solchen Entscheidung nämlich nicht sagen können, welche Option besser ist, können wir im Grunde genommen nicht angeben, für welche Option wir die besten Gründe besitzen. Diesem Verständnis von Rationalität liegt die Intuition zugrunde, dass eine begründete und damit rationale Entscheidung eine solche ist, welche die in den zur Wahl stehenden Handlungsoptionen relevanten Werte oder wertvollen Eigenschaften erschöpfend - und dies bedeutet im Sinne der Trichotomie-These in einer der drei Relationen - zueinander in Beziehung setzt. Ein Akteur scheint dann keine rationale Entscheidung treffen zu können, wenn Handlungsoptionen aufgrund ihrer Unvergleichbarkeit nicht als besser, schlechter oder gleich gut bewertet werden können.

Etliche Philosophinnen und Philosophen sind daher der Meinung, dass harte Fälle genuine Konflikte darstellen, weil die zur Wahl stehenden wertvollen Optionen unvergleichbar sind (vgl. etwa Raz 1986 und Boot 2009). In der Folge haben Vertreter und Vertreterinnen der Unvergleichbarkeitsdebatte allerdings verschiedene Möglichkeiten eruiert, die harte Fälle als nur scheinbar unvergleichbar ausweisen (siehe z. B. Broome 1997, Chang 2002a und Hsieh 2005).

Nicht zuletzt im Hinblick auf bestehende Rationalitätstheorien wäre Vergleichbarkeit eine notwendige Voraussetzung für die Rationalität der Entscheidung. Inwiefern wir aber tatsächlich sehr unterschiedliche, unvergleichbar scheinende Handlungsoptionen vergleichen und uns in solchen Entscheidungssituationen rational verhalten können oder ob die These, dass wir dabei eine rationale Wahl treffen können, aufgrund von unvergleichbaren Handlungsoptionen und harten Fällen nicht vielmehr einen Mythos darstellt, ist Gegenstand der Inkommensurabilitätsdebatte.

In diesem Buch interessieren im Wesentlichen zwei Punkte aus dieser Kontroverse. Erstens die Annahme, dass harte Fälle Situationen darstellen, in denen die Optionen unvergleichbar sind, 
und zweitens die Behauptung, dass es keine rationale Entscheidung zwischen unvergleichbaren Optionen gibt. Dabei werden beide Thesen untersucht. Es geht im Einzelnen darum, (i) Unvergleichbarkeit und harte Fälle zu verstehen (Was ist Unvergleichbarkeit?), (ii) den Zusammenhang mit der rationalen beziehungsweise irrationalen Entscheidungsfindung aufzuzeigen (Worin besteht das Problem für die Rationalität?) und (iii) Möglichkeiten zu diskutieren, was im Falle von unvergleichbaren Handlungsoptionen zu tun ist (Wie kann damit umgegangen werden?).

\section{Worin besteht das Problem?}

Zusammenfassend kann das Problem harter Fälle in Form eines Arguments folgendermassen dargestellt werden (gemäss meinem Wissen wurde das Problem bislang nicht in dieser Weise auf den Punkt gebracht):

1. Die Vergleichbarkeit der Handlungsoptionen stellt eine notwendige Bedingung einer rationalen Wahl dar.

2. Zwei Optionen, $x$ und $y$, sind genau dann vergleichbar, wenn $x$ entweder besser oder schlechter als $y$ oder $x$ und $y$ gleich gut sind.

3. Es gibt harte Fälle, in welchen $x$ weder besser oder schlechter als $y$ ist noch $x$ und $y$ gleich gut sind.

$\therefore \quad$ Es gibt harte Fälle, in welchen keine rationale Entscheidung möglich ist.

Prima facie sind die Prämissen des obigen Arguments plausibel: Wie können wir zwischen Alternativen wie Anwältin respektive Klarinettistin werden rational entscheiden, wenn wir die Optionen nicht vergleichen können? Wie können wir zwei Optionen vergleichen, wenn diese weder besser oder schlechter als noch gleich gut wie die jeweilig andere Option sind? Die Konklusion, welche daraus folgt - in harten Fällen ist keine rationale Wahl möglich -, stellt insofern eine problematische Konsequenz dar, als wir grundsätzlich ein Interesse daran haben, rationale Entscheidungen zu treffen und 
unsere Entscheidungen vor uns selbst (und somit auch vor anderen) rechtfertigen zu können.

\section{Was ist das Ziel?}

Vor dem Hintergrund der bisherigen philosophischen Forschung sollen folgende fünf Fragen präziser gestellt und beantwortet werden.

1. Wie lässt sich Unvergleichbarkeit genau verstehen?

2. Wie können harte Fälle angemessen interpretiert werden?

3. Inwiefern ist eine rationale und damit begründete Entscheidung in harten Fällen möglich?

4. In welchem Verhältnis stehen Rationalität und Vergleichbarkeit?

5. Was ist im Falle von unvergleichbaren Handlungsoptionen zu tun?

Das Ziel besteht darin, eine neue Interpretation von Unvergleichbarkeit und harten Fällen vorzuschlagen und mögliche Wege aufzuzeigen, wie damit umgegangen werden kann. Es wird eine neue Präferenzversion entwickelt und verteidigt. Zwei Optionen sind demnach genau dann unvergleichbar, wenn eine Akteurin keine Präferenz konstruieren kann - im Sinne einer Evaluation, welche Option alles in allem besser ist. Hierbei ist entscheidend, wie man zu einer Präferenz kommt respektive wie man nicht dazu kommt. Ich argumentiere, dass evaluatives Unwissen das zentrale Argument für Unvergleichbarkeit darstellt. Diese Unwissenheit über Werte oder Gründe hat nicht zwingend mit Ethik zu tun. Sie meint allgemeiner, dass man nicht weiss, wie Fakten bewertet werden sollen. Beispielsweise weiss ein Akteur nicht, wie der Fakt, dass es leicht regnet, zu bewerten ist. Die Unwissenheit ist also insofern evaluativ, als sie sich weder auf Fakten (Regnet es?) noch darauf bezieht, was passieren wird (Wird es regnen?), sondern darauf, welche Gründe von Fakten generiert werden. Stellt leichter Regen einen Grund dar, einen Regenschirm einzupacken, oder stellt es keinen Grund dafür dar? 
Wie ist das Buch aufgebaut?

Das Buch gliedert sich in fünf Teile (10 Kapitel):

1. Der erste Teil beinhaltet begriffliche Klärungen (Kapitel 1).

2. Im zweiten Teil wird das Phänomen der Unvergleichbarkeit erfasst. Es wird eine eigene Theorie von Unvergleichbarkeit und harten Fällen entwickelt; diese werden von benachbarten Phänomenen abgegrenzt. Hierbei werden Argumente für Unvergleichbarkeit genannt und Argumente dagegen zurückgewiesen (Kapitel 2, 3, 4, 5).

3. Im dritten Teil wird erläutert, worin genau die Herausforderung an die Rationalität besteht (Kapitel 6).

4. Im vierten Teil werden bestehende Versuche studiert, harte Fälle als lediglich scheinbar unvergleichbar beziehungsweise rational auszuweisen (Kapitel 7, 8, 9).

5. Im fünften Teil und letzten Kapitel werden mögliche Strategien diskutiert, wie mit Unvergleichbarkeit umgegangen werden kann (Kapitel 10).

\section{Welches ist die These?}

Ich vertrete drei Thesen. Die erste beantwortet die Frage, was Unvergleichbarkeit ist. Die zweite bezieht sich darauf, ob ein Akteur zwischen unvergleichbaren Optionen rational entscheiden kann. Die dritte betrifft die Frage, wie wir mit Unvergleichbarkeit umgehen können.

Was ist Unvergleichbarkeit? Die vorgeschlagene Theorie besagt, dass sich das Phänomen der Unvergleichbarkeit in drei wesentliche Typen unterteilen lässt (diese sind notabene nicht disjunkt; vgl. Kapitel 3). Der einfachste harte Fall beinhaltet einen Wertkonflikt, was so interpretiert werden kann, dass man nicht weiss, wie hoch man unterschiedliche Werte schätzt (evaluatives Unwissen). ${ }^{2}$ Der zweite harte

2 Man mag einwenden, dass diese Hauptthese der Arbeit unvereinbar ist mit einer 'realistischen' Auffassung, die von einem realen Wert von Optionen ausgeht (und nicht dem Wert, der eine Person einer Option zubilligt). Ich diskutiere diese Kritik in Kapitel 2 und Kapitel 3. 
Fall involviert evaluatives Unwissen und stellt zusätzlich eine wichtige Wahl dar. Dies kann so gedeutet werden, dass damit viele neue Optionen ermöglicht respektive verunmöglicht werden. Der dritte und schwierigste harte Fall beinhaltet evaluatives Unwissen, ist wichtig und zusätzlich mit faktischer Unsicherheit verbunden. Damit ist gemeint, dass man nicht weiss, was nach der getroffenen Entscheidung passieren wird, und dies unter Umständen für die Evaluation relevant ist. Die erste These lautet somit wie folgt: Harte Fälle sind respektive Unvergleichbarkeit ist real und kann nicht als Randphänomen oder sogar als inexistent abgetan werden. Alle Fälle von Unvergleichbarkeit können auf evaluatives Unwissen zurückgeführt werden, viele sind zusätzlich wichtig und/oder mit faktischer Unsicherheit verbunden. Bei allen drei Typen kann eine Akteurin keine Präferenz bilden.

Ist im Falle von Unvergleichbarkeit eine rationale Wahl möglich? Wie im Abschnitt «Worin besteht das Problem?» festgehalten worden ist, sind es drei plausibel scheinende Prämissen, welche zur problematischen Konsequenz führen, dass wir in harten Fällen nicht rational entscheiden können: Erstens die Annahme, dass die Vergleichbarkeit der Handlungsoptionen eine notwendige Bedingung einer rationalen Wahl darstellt. Zweitens die Auffassung, dass zwei Optionen, $x$ und $y$, nur dann vergleichbar sind, wenn $x$ entweder besser oder schlechter als $y$ ist oder $x$ und $y$ gleich gut sind. Drittens die Ansicht, dass in harten Fällen die Optionen weder besser oder schlechter als die jeweilig andere Option noch gleich gut sind. In dieser Arbeit komme ich zum Schluss, dass diese Prämissen nicht nur plausibel erscheinen, sondern es auch sind. Bisherige Versuche zur Lösung des Problems beschreiben harte Fälle entweder nicht angemessen oder können, sofern es ihnen um neue Möglichkeiten der Vergleichbarkeit scheinbar unvergleichbarer Fälle geht, nicht hinreichend zeigen, wie diese rational zu lösen sind. Sowohl Positionen, welche die zweite Annahme in Zweifel ziehen - Handlungsoptionen können auch dann verglichen werden, wenn sie weder als besser oder schlechter als noch als gleich gut wie bewertet werden können (Chang 2002a) - als auch Positionen, welche die dritte Annahme ablehnen - das heisst im Grunde genommen die Existenz harter Fälle verneinen (Regan 1997) - können nicht überzeugen. Dazu kommt, dass Vergleichbarkeit zwar nicht notwendigerweise, aber in den für uns relevanten Fällen und 
unter bestimmten Bedingungen als Voraussetzung einer rationalen Entscheidung angesehen werden kann. (Ich zeige, dass Vergleichbarkeit aus verschiedenen Gründen nicht notwendig für Rationalität ist. Nicht alle Konzepte der Rationalität setzen Vergleichbarkeit voraus. Auch kann das Vergleichen von Dingen sehr problematisch sein. Und nicht zuletzt ist es eine offene Frage, ob Optionen manchmal, nämlich wenn spezifische Dinge wie Freundschaft, Liebe, oder Loyalität involviert sind, nicht verglichen werden sollen.) Die zweite These lautet dementsprechend folgendermassen: Unvergleichbarkeit lässt sich punktuell nicht rational auflösen. Das Problem für die Rationalität besteht dabei darin, dass die 'Lücke' zwischen den möglichen und gewählten Alternativen nicht mit einer Präferenz respektive durch die Angabe von Gründen oder Werten geschlossen werden kann. Weil diese Art der Evaluation versagt, lassen sich die Optionen in keine begründete Ordnung bringen.

Wie kann damit umgegangen werden? Die Arbeit grenzt sich von der bisherigen philosophischen Forschung in dem Sinne ab, als der Fokus pragmatischer und alltagsnaher ausfällt (Was tun wir jetzt?). Diese Frage ist bislang kaum diskutiert worden (eine Ausnahme stellt Chang $2013 \mathrm{~b}$ dar; vgl. Kapitel 10). Dabei gelange ich zur eher überraschenden Erkenntnis, dass es bei einem bestimmten Typus von Unvergleichbarkeit und unter bestimmten Bedingungen - zum Beispiel faktische Unsicherheit ist vernachlässigbar, Kohärenz nicht ausschlaggebend (Keine Option passt besser ins Leben) - schlicht keine Rolle spielt, ob aufgrund einer nicht-begründeten Neigung (Mir gefällt es irgendwie besser), einer existenziellen Stellungnahme (Ich sehe mich als Musikerin) oder einer beliebigen Wahl entschieden wird. Das Argument, welches ich für meine These anfüge, basiert auf der Idee, dass Wohlergehen eine Begleiterscheinung einer beliebigen Tätigkeit, eine Form der Zufriedenheit darstellt, welche sich einstellt oder ausbleibt, wenn man Ziele verfolgt und Dinge tut. Was hat dies mit beliebigem Wählen zu tun? Die Idee ist, dass sich die Unvergleichbarkeit nur durch ein Hineindenken und letztlich ein Ausprobieren beziehungsweise durch Erfahrung 'auflösen' lässt. Wenn man einen Monat, nachdem man mit dem Hockeyspielen aufgehört hat, realisiert, dass sich das Wohlergehen verschlechtert, man irgendwie unzufrieden ist, stellt dies einen Indikator dafür dar, dass es die falsche Entscheidung war, stattdessen 
im Chor mitzumachen. Inwiefern eine Entscheidung gut ist, kann gemäss diesem Ansatz nicht punktuell beurteilt werden. Es kann sich nur mit der Zeit zeigen. Das heisst, dass die 'Auflösung' einer evaluativen Unsicherheit in der Regel einen Lernprozess voraussetzt, um zu erfahren, was zählt. Deshalb ist ein Akteur nicht dazu aufgefordert, in bestimmter Weise zu wählen oder sich zu etwas zu bekennen, sondern in bestimmter Weise zu handeln. Die dritte These besagt demnach, dass wir unter gewissen Voraussetzungen beliebig wählen dürfen. Intuitiv gehen wir eher vom Gegenteil aus. Wir denken, dass insbesondere wichtige Entscheidungen nicht zufällig aufgelöst werden sollten.

Halten wir fest: Dieses Buch stellt zwei Grundsätze in Frage: jenen, dass wir in harten Fällen rational wählen können - diese Position wird etwa von Andreou 2005 vertreten - und jenen, dass wir immer selbst diese Wahl treffen müssen (contra Chang 2002b). Die Entscheidung zwischen unvergleichbaren Optionen ist oft nicht rational und darf manchmal dem Würfel überlassen werden. Wir kommen schlicht nicht umhin, uns in solchen Situationen auf Handlungsweisen einzulassen, deren Wert und Ausgang für uns ungewiss sind. 
Kapitel 1

Inkommen-

surabilität und

Unvergleich-

barkeit 
Das Ziel dieses Kapitels besteht darin, dass nachvollziehbar wird, was genau an Unvergleichbarkeit real ist, welche Konsequenzen dies für den weiteren Verlauf des Buches hat und welches Phänomen und welche Argumente in den folgenden Kapiteln untersucht werden. Die erste wichtigste Erkenntnis lautet, dass den Begriffen der Unvergleichbarkeit und Inkommensurabilität nicht ein einziges Phänomen zugrunde liegt. Das Kapitel gliedert sich wie folgt:

Im ersten Teil wird dargelegt, inwiefern die Begriffe der Inkommensurabilität und Unvergleichbarkeit sehr unterschiedliche Verwendungsweisen aufweisen.

Im zweiten Teil zeige ich, dass mithilfe einer klassischen Begriffsanalyse weder notwendige noch hinreichende Bedingungen von Inkommensurabilität und Unvergleichbarkeit ausgemacht werden können. Der Grund dafür ist, dass es keine typischen Situationen respektive paradigmatischen Fälle der Inkommensurabilität gibt. Zudem erläutere ich, weshalb auch die Suche nach Familienähnlichkeiten nicht zielführend ist.

Im dritten Teil stelle ich dar, in welchem Sinne ich eine Begriffsexplikation vornehme und weshalb dies kompatibel damit ist, dass eine Definition von Unvergleichbarkeit reale Komponenten besitzt.

\subsection{Die Begriffe der Inkommensurabilität und Unvergleichbarkeit}

Was ist wertvoller, ein besseres Verständnis wirtschaftlicher oder juristischer Zusammenhänge? Sollen bei der Studienwahl das momentane Interesse oder die späteren Berufsaussichten stärker gewichtet werden? Und welches stellt alles in allem das bessere Studium dar?

Die Begriffe der Inkommensurabilität und Unvergleichbarkeit, die für uns relevant sind, haben mit Werten, Gründen und Handlungsoptionen zu tun. In diesem Zusammenhang kann man vertreten, dass die Wahl zwischen den unterschiedlichen Werten und Gründen (wirtschaftliche versus juristische Erkenntnis) bis zu einem gewissen Grade willkürlich sei. In der Konsequenz heisst dies dann, dass bis zu einem gewissen Grade zufällig ist, was man tun soll (Wirtschaft oder Rechtswissenschaft studieren). 
Bevor zwischen verschiedenen Begriffsverwendungen innerhalb der praktischen Philosophie differenziert wird, soll kurz betrachtet werden, in welchen anderen Disziplinen die Begriffe der Inkommensurabilität und Unvergleichbarkeit Anwendung finden und weshalb dies für uns nicht von Bedeutung ist.

Unvergleichbarkeit und Inkommensurabilität spielen nämlich nicht nur in der praktischen Philosophie (inklusive Anwendung auf Ethik ${ }^{1}$ ), sondern auch in anderen Gebieten eine Rolle. Dementsprechend ist dasjenige, worauf sich die beiden Begriffe beziehen, mannigfaltig.

Neben der Jurisprudenz ${ }^{2}$ und Physik ${ }^{3}$ kommen sie etwa in der Mathematik vor. In dieser Disziplin taucht der Begriff der Inkommensurabilität erstmals auf. Inkommensurable Zahlen wie die Eulersche Zahl oder die Zahl Pi stellen keine rationalen Zahlen dar, sind also Zahlen, die sich nicht als Bruch notieren lassen. Die Inkommensurabilität in der Mathematik geht auf die alten Griechen zurück. Waschkies dokumentiert in seinem Buch Anfänge der Arithmetik im alten Orient und bei den Griechen die Entdeckung inkommensurabler Grössen in der griechischen Mathematik. Die Inkommensurabilität bezieht sich dabei auf geometrische Figuren wie ein Fünfeck (Waschkies 1989: 310, 311, 315) oder ein Quadrat (Waschkies 1989: 308, 309, 310). Bestimmte Seiten und Diagonalen in diesen Figuren können inkommensurabel sein. Das heisst, dass ihr Verhältnis nicht als Bruch beziehungsweise nicht als Verhältnis natürlicher Zahlen dargestellt werden kann. Das Verhältnis von Diagonale und Seite ist eine irrationale Zahl. Man kann dies mit Methoden der aktuellen Mathematik anhand eines Reductio-Beweises zeigen. Zu diesem Zweck geht man (i) von der Prämisse aus, dass das Verhältnis von Diagonale und Seite eine rationale Zahl sei, leitet daraus (ii) einen Widerspruch ab und führt damit (iii) die angenommene Prämisse ad absurdum (das Gegenteil der Prämisse ist wahr beziehungsweise das Verhältnis stellt eine irrationale Zahl dar).

1 Vgl. z. B. Sinnott-Armstrong 1985 (Anwendung auf moralische Dilemmata), Boot 2007 (Anwendung auf Gerechtigkeit) und Peterson und Fröding 2012 (Anwendung aufTugendethik).

$2 \quad$ Siehe etwa Sunstein 1997 und Adler 1998.

3 In der Physik können es mathematische Kalküle sein, welche inkommensurabel sind. Damit sind Systeme gemeint, die aus Axiomen und Schlussregeln bestehen und sich nicht ineinander übersetzen lassen. vgl. z. B. Waschkies 1989. 
Inkommensurabilität spielt aber auch in der Wissenschaftstheorie eine wichtige Rolle. ${ }^{4}$ Inkommensurabilität und Unvergleichbarkeit werden im Zusammenhang mit wissenschaftlichen Theorien oder Paradigmen diskutiert. Inkommensurable wissenschaftliche Theorien setzen unterschiedliche konzeptuelle Bedingungen voraus. Damit ist gemeint, dass sich inkommensurable Theorien nicht anhand der jeweilig anderen Theorie verstehen oder bewerten lassen. Als Beispiel wird manchmal die Newtonsche und die Einsteinsche Theorie von Raum und Zeit erwähnt. Während Newton von einem 'absoluten Raum' und einer 'absoluten Zeit' ausgeht, müssen Raum und Zeit in der Relativitätstheorie als Einheit (als 'Raumzeit') verstanden werden. Diese besteht nicht unabhängig von der Materie - im Gegensatz zu Newtons Konzept von Raum und Zeit - und setzt sich aus drei räumlichen und einer vierten zeitlichen Dimension zusammen.

In diesem Zusammenhang kann man argumentieren, dass die Wahl zwischen den unterschiedlichen Paradigmen oder Theorien bis zu einem gewissen Grade willkürlich sei. In der Konsequenz heisst dies dann, dass unsere Beschreibung und Erklärung der Welt (Was ist der Fall?) einigermassen zufällig ist.

$\mathrm{Ob}$ es einen allgemeinen Begriff von Unvergleichbarkeit und Inkommensurabilität gibt, der allen oben genannten Bereichen zugrunde liegt, ist fraglich. Und selbst wenn es einen solchen Begriff gäbe, wäre er aufgrund einer zu grossen Extension nichtssagend. Wenn nämlich zu viele Dinge unter einen Begriff fallen, wird dieser 'leer'. Hinsichtlich der zentralen Frage dieser Arbeit - Welche Konsequenz hat Unvergleichbarkeit für die rationale Entscheidungsfindung? - sind die Begriffe der Inkommensurabilität aus den Bereichen ausserhalb der praktischen Philosophie allerdings nur sehr beschränkt oder überhaupt nicht relevant. Beispiele, in denen eine Wissenschaftlerin aufgrund inkommensurabler Theorien oder Paradigmen keine rationale Entscheidung treffen kann, sind unter Umständen denkbar. So ist es möglich, dass zwei Ökonomen bei der Beantwortung einer Frage a priori entweder einem neoklassischen oder einem keynesianischen Paradigma folgen und somit keine rationale Entscheidung treffen können, in dem Sinne, dass es nicht wohlbegründet oder nicht

4 Die prominenten Positionen findet man etwa in Kuhn 2012 und Feyerabend 1997. Einen Überblick über die Debatte liefert beispielsweise D’Agostino 2014. 
gerechtfertigt ist, das eine oder andere Paradigma zu wählen. Aber in diesem Zusammenhang, so meine ich, wären etliche Annahmen nötig, wie zum Beispiel, dass sich die relevante Frage nicht anhand von Daten entscheiden lässt. Man müsste argumentieren, dass der neoklassisch geprägte Ökonom und der keynesianisch geprägte nicht einfach hinschauen könnten, was geschieht. Sie müssten beispielsweise (i) unterschiedliche Messmethoden akzeptieren, (ii) unterschiedliche (theorieabhängige) Beobachtungen vornehmen oder (iii) eine verschiedene Interpretation derselben Daten wählen. Die Tatsache hingegen, dass sich die Eulersche Zahl nicht als Bruch notieren lässt, spielt für die Beantwortung dieser Frage überhaupt keine Rolle.

Auch innerhalb des spezifischen Bereichs der praktischen Philosophie werden die Begriffe sehr unterschiedlich verstanden und nicht einheitlich definiert. In diesem Zusammenhang lassen sich mindestens zehn Definitionen von Inkommensurabilität und Unvergleichbarkeit unterscheiden: (i) zwei Optionen sind nicht besser/schlechter/ gleich gut, (ii) ein Akteur präferiert keine Alternative, (iii) ein Akteur darf/soll keine Alternative präferieren, (iv) zwei Optionen sind kardinal, aber nicht ordinal vergleichbar, (v) zwischen zwei Gegenständen besteht keine positive Wertrelation, (vi) zwei Werte/Gegenstände lassen sich nicht hinsichtlich eines allgemeinen Deckwerts vergleichen, (vii) zwei Werte/Gegenstände lassen sich nicht hinsichtlich einer numerischen Skala (quantitativ) vergleichen, (viii) zwischen zwei Standards ist keine Substitution möglich, (ix) zwei Werte/Gegenstände sind konstitutiv unvergleichbar (konstitutive Inkommensurabilität), (x) alternative Definition: zwei Werte sind inkompatibel; keine moralische Norm ist 'stärker' u. a. Ich werde die verschiedenen Ansätze im Folgenden in der genannten Reihenfolge erläutern.

Zwei Handlungsoptionen/Werte/Gegenstände, $x$ und $y$, sind nach Ansicht einiger Philosophen genau dann inkommensurabel, wenn $x$ und $y$ unvergleichbar sind. ${ }^{5}$ Meistens wird dies so verstanden, dass $x$ und $y$ nicht besser/schlechter/gleich gut sind ${ }^{6}$ Genauer 1986: 322, Griffin 1997: 35 (in Griffin 1986: 83 wird eine Gleichsetzung abgelehnt), Lukes 1997: 184 und Kavka 1991: 147.

6 Vgl. Andreou 2005: 249, Raz 1986: 322, Raz 1997: 110, Anderson 1997: 90, Rabinowicz 2009: 71, Constantinescu 2012: 58. 
gesagt sind die beiden Aussagen genau dann gleichbedeutend, wenn die sogenannte Trichotomie-These richtig ist. Vereinfacht gesagt meint diese These, dass drei Vergleichsrelationen existieren: ist besser als, ist schlechter als und ist gleich gut wie. ${ }^{7}$ Das bedeutet, dass $x$ und $y$ genau dann unvergleichbar respektive inkommensurabel sind, wenn $x$ weder besser noch schlechter als noch gleich gut wie $y$ ist. Dies stellt die gängigste Definition dar. Konkret bedeutet dies beispielsweise, dass ein Studium der Jurisprudenz genau dann unvergleichbar mit einem Studium der Ökonomie ist, wenn die beiden Optionen weder besser noch schlechter als noch gleich gut wie die jeweilig andere Option sind.

In diesem Zusammenhang wird meistens keine weitere Erläuterung hinzugefügt, was mit besser als oder gleich gut wie gemeint ist. Je nachdem, was dies bedeutet, können weitere Präzisierungen vorgenommen werden. Unter den bestehenden Erläuterungen können folgende zwei Möglichkeiten unterschieden werden: besser als verstanden als Präferenz versus besser als verstanden als Präferenz, die man haben soll/darf. Das heisst: Zwei Optionen sind genau dann unvergleichbar, wenn ein Akteur weder eine Präferenz besitzt noch indifferent hinsichtlich der zur Wahl stehenden Alternativen ist (vgl. etwa Morton 1991: 32). Ein Studium der Ökonomie ist demnach genau dann unvergleichbar mit einem Studium der Jurisprudenz, wenn man weder eine Präferenz für Ökonomie noch für Jurisprudenz besitzt und auch nicht indifferent gegenüber den beiden Studiengängen ist. Und: Zwei Optionen sind genau dann unvergleichbar, wenn ein Akteur weder eine Präferenz besitzen noch indifferent sein soll. Ein Studium der Ökonomie ist demnach genau dann unvergleichbar mit einem Studium der Jurisprudenz, wenn man weder eine Präferenz für Ökonomie noch für Jurisprudenz haben und auch nicht indifferent sein soll. ${ }^{8}$ Und: Zwei Optionen sind genau dann unvergleichbar, wenn ein Akteur irgendeine Präferenz haben oder indifferent sein darf (vgl. Rabinowicz 2008: 27). Dies kann auch so gedeutet werden, dass

$7 \quad$ Vgl. Kapitel 7 für eine exakte Definition und Diskussion der Trichotomie-These.

8 Diese Definition von Unvergleichbarkeit wird von Broome 1991: 93, Gustafsson und Espinoza 2010: 754 und Carlson 2011: 171 vertreten. Rabinowicz formuliert es wie folgt: ' $x$ and $y$ are incomparable if and only if it is required not to prefer one to the other or to be indifferent.' (Rabinowicz 2009: 84). Dies kann auch so interpretiert werden, dass die Optionen in keine ordinale Rangordnung gebracht werden sollen. Kornhauser nennt dies 'Radical Incommensurability' (Kornhauser 1998: 1604). 
es erlaubt ist, unterschiedliche ordinale Reihenfolgen der beiden Studiengänge vorzunehmen. Kornhauser nennt dies 'Order Incommensurability' (Kornhauser 1998: 1604).

Gemäss einer weiteren Definition sind $x$ und $y$ genau dann inkommensurabel, wenn sie nicht kardinal verglichen werden können (Stocker 1997: 203). Inkommensurabilität meint dementsprechend, dass es Vergleiche gibt, die ordinal, aber nicht kardinal möglich sind. Chang geht von einem solchen Verständnis von Inkommensurabilität aus. ${ }^{9}$

Um genauer zu verstehen, was damit gemeint ist, müssen wir einen kurzen Blick in die Entscheidungstheorie werfen. Die Ordinalkardinal-Unterscheidung spielt in der Entscheidungstheorie eine wichtige Rolle. Diese Differenzierung kommt dann ins Spiel, wenn es darum geht, wie man das Konzept des Nutzens interpretieren soll. Nehmen wir an, dass die Option 'Ökonomie' vier Nutzen-Punkte und die Option 'Jurisprudenz' zwei Nutzen-Punkte generiert. In einem ordinalen Nutzensystem kann man in diesem Fall sagen, dass es besser ist, Ökonomie zu studieren, aber nicht, um wie viel es besser ist. In einem ordinalen Nutzensystem sagt die Zahl 2 nur, dass Ökonomie besser ist. Wenn der Nutzen ordinal skaliert ist, ist lediglich die Reihenfolge von Bedeutung. Den Zahlen kommt keine weitere Relevanz zu. Man kann sagen, dass ordinaler Nutzen nichts anderes als eine Präferenzordnung darstellt. In einem kardinalen Nutzensystem kommt ein zusätzlicher Schritt hinzu. Die Zahl 2 hat eine andere Bedeutung. Die Interpretation ist nicht so einfach, dass man dies so deuten kann, dass es in einem kardinalen Nutzensystem genau doppelt so gut ist, Ökonomie zu studieren. Die Ordinal-kardinalUnterscheidung ist ohne Mathematik nicht nachvollziehbar: Jede ordinale Nutzenfunktion kann positiv monoton transformiert werden,

9 Im Sammelband Incommensurability, Incomparability and Practical Reason hält sie fest: '(...) let us henceforth reserve the term 'incommensurable' for items that cannot be precisely measured by some common scale of units of value.' (Chang 1997: 2). Im Sammelband von 1997 ist noch nicht eindeutig rekonstruierbar, was Chang damit meint. In späteren Texten, insbesondere in Incommensurability (and Incomparability) wird aber deutlich, dass Chang unter Inkommensurabilität wie Stocker kardinale Unvergleichbarkeit versteht: '(...) one concerning what is properly called 'incommensurability' or the lack of a cardinal unit by which values can be measured (...)’ (Chang 2013a: 2601). Inkommensurabilität wird in Anlehnung an Chang denn auch als kardinale Unvergleichbarkeit verstanden (siehe z. B. Aldred 2013: 133). 
jede kardinale Nutzenfunktion hingegen nur linear. Ich kann dies umgangssprachlich nicht einfacher erläutern, als dass die Frage 'Wie viel besser ist ein Studium der Ökonomie als ein Studium der Jurisprudenz?' bei einem kardinalen Vergleich präziser beantwortet wird. Wenn der Nutzen kardinal skaliert ist, enthält die Differenz, die Zahl 2, mehr Informationen. Welche dies sind, wird intuitiv erst nachvollziehbar, wenn die Entscheidungssituation um eine dritte Option, ein Studium der Musik etwa, erweitert wird. Nehmen wir an, dass ein Studium der Musik 12 Nutzen-Punkte generiert. Es liegen also drei Optionen vor, die ein unterschiedliches Nutzenniveau erzeugen: Jurisprudenz (2), Ökonomie (4) und Musik (12). In einem ordinalen Nutzensystem ist Musik dementsprechend besser als Ökonomie und Ökonomie besser als Jurisprudenz. Mehr wird damit nicht ausgesagt, und deshalb dürfen die Zahlen beliebig monoton transformiert werden - zum Beispiel darf jede Nutzenfunktion mit $a$ multipliziert werden und $b$ darf hinzu addiert werden. Dadurch wird die Reihenfolge nicht verändert. In einem kardinalen System hat der Abstand eine zusätzliche Bedeutung. Der Abstand zwischen Musik und Ökonomie ist grösser als der Abstand zwischen Ökonomie und Jurisprudenz. Musik ist 'mehr besser als' Ökonomie. Deshalb dürfen die Zahlen nur linear transformiert werden - zum Beispiel darf zu jeder Nutzenfunktion 2 addiert werden, so dass Jurisprudenz dann 4, Ökonomie 6 und Musik 14 Nutzen-Punkte generiert. Damit bleibt nicht nur die Reihenfolge, sondern auch der Abstand erhalten. Man spricht in diesem Zusammenhang davon, dass kardinaler Nutzen 'ratio preserving' ist, im Gegensatz zu ordinalem Nutzen, der nur 'order preserving' ist. Die zusätzliche Information beim kardinalen Vergleich besteht also darin, dass ein Akteur Musik gegenüber - relativ gesehen - eine wesentlich höhere Wertschätzung zeigt. Auch in einem kardinalen Nutzensystem kann diese höhere Wertschätzung aber nicht so präzise verstanden werden, dass sie beispielsweise doppelt oder dreifach so hoch ist. Die Differenz zwischen ordinaler und kardinaler Vergleichbarkeit umfasst viele Implikationen. Die wichtigste besteht wohl darin, dass bei einem kardinalen Vergleich die Differenzen im Nutzen vergleichbar sind. Demzufolge impliziert Inkommensurabilität (es ist kein kardinaler Vergleich möglich), dass die Differenzen im Nutzen nicht verglichen werden können. 
Eine andere Definition von Unvergleichbarkeit besagt, dass zwischen den Gegenständen keine positive Wertrelation besteht. Chang, welche die Trichotomie-These ablehnt ${ }^{10}$, vertritt diese Meinung: 'Two items are incomparable if no positive value relation holds between them.' (Chang 1997: 4). Ein Studium der Ökonomie ist demnach genau dann unvergleichbar mit einem Studium der Jurisprudenz, wenn zwischen beiden Studiengängen keine positive Wertrelation besteht. In dieser Definition wird sowohl offengelassenen, was eine Wertrelation ist, als auch, wie viele Vergleichsrelationen es gibt. Ergo fällt die Definition auch ungenau aus.

Inkommensurabilität wird oft auch so gedeutet, dass zwei Werte oder Gegenstände nicht präzise verglichen werden können, in dem Sinne, dass es keine Möglichkeit der Beurteilung hinsichtlich eines gemeinsamen Standards gibt (Boot 2007: 24). Oder mit Grimm formuliert: 'Value incommensurability is the claim that there is no common measure in terms of which values can be weighted.' (Grimm 2007: 27). Inkommensurabilität meint dementsprechend nicht, dass zwei Dinge überhaupt nicht verglichen werden können, sondern, dass kein sogenannter Deckwert existiert (vgl. z. B. Chang 2002a: 666). Je nachdem, was unter einem Deckwert verstanden wird, können hierbei zwei Definitionen unterschieden werden. Einerseits kann ein Deckwert ein numerisches Kriterium darstellen. Inkommensurabilität hat dann nicht mit Werten, sondern mit quantitativ messbaren Grössen wie Gewicht, Körperlänge oder Anzahl produzierter Schrauben zu tun. Ein Beispiel: Hans ist hinsichtlich Körpergrösse genau dann unvergleichbar mit Simon, wenn er weder grösser noch kleiner als noch gleich gross wie Simon ist (weil das nicht der Fall ist, sind die beiden vergleichbar). Oder: Maria hat unter ähnlichen Arbeitsbedingungen und im gleichen zeitlichen Rahmen wie Hans und gemäss bestimmten Qualitätsansprüchen 80 Schrauben hergestellt. Hans schaffte dagegen nur 50 Stück. Maria hat in dieser Sequenz also anscheinend mehr geleistet. Das Produkt ihrer Handlung war gemäss einer einfachen numerischen Leistungsskala besser. Wenn bei einem Vergleich jedoch kein numerisches Kriterium herbeigezogen werden kann, Hans anstatt Schrauben anzufertigen in dieser Zeit eine Website

10 Vgl. Chang 2015a: 18. Damit meine ich, dass Chang sowohl die Trichotomie-These als auch die Erklärung von Inkommensurabilität mithilfe der Trichotomie-These ablehnt. 
programmiert hat und Maria währenddessen einen Tisch anfertigte, sind ihre Arbeitsleistungen inkommensurabel. Andererseits kann ein Deckwert aber auch breiter verstanden werden. Die unterschiedlichen Werte, die im Falle des Studiums der Ökonomie - zum Beispiel ein besseres Verständnis wirtschaftlicher Zusammenhänge - und im Falle des Studiums der Jurisprudenz - zum Beispiel ein besseres Verständnis des Rechtssystems - realisiert werden, lassen sich in diesem Fall nicht hinsichtlich eines gemeinsamen Werts wie 'interessantes Studium' vergleichen. Der Standard 'interessantes Studium' stellt jedoch kein numerisches Kriterium wie 'Anzahl produzierter Schrauben' dar.

Gemäss einer alternativen Sichtweise von Inkommensurabilität, welche D’Agostino vertritt, ist zwischen zwei Standards keine Substitution beziehungsweise kein trade-off möglich. D’Agostino unterscheidet u. a. zwei Typen von Inkommensurabilität: sogenannte L- und S-Inkommensurabilität. Im Falle der S-Inkommensurabilität ist keine Abwägung möglich, weil keine sogenannte determinate rate of substitution existiert (D’Agostino 2003: 43). D'Agostino verwendet diesen Begriff gleich, wie es in der Mikroökonomie üblich ist. Damit ist gemeint, dass beispielsweise nicht angegeben werden kann, wie die Standards Arbeit und Freizeit substituiert werden sollen. Es kann etwa nicht gesagt werden, dass zwei Stunden Freizeit gegen eine Stunde Arbeit getauscht werden sollen. In einem mikroökonomischen Modell ist dies gleichbedeutend damit, dass sich für die beiden Güter keine Indifferenzkurve zeichnen lässt. Im Falle der L-Inkommensurabilität ist keine Abwägung möglich, weil eine infinite Substitutionsrate existiert. Das heisst nichts anderes, als dass ein Gut oder Standard lexikographisch vorgezogen wird. L-Inkommensurabilität meint eine lexikographische Priorisierung (das eine ist immer besser als das andere). Zum Beispiel ist Freizeit immer besser als Arbeit. Dies wiederum kann so verstanden werden, dass, unabhängig davon, wie viel durch die Arbeit mehr verdient wird, das Defizit an Freizeit niemals kompensiert werden kann. Der Unterschied zwischen den beiden Typen von Inkommensurabilität besteht nach D'Agostino darin, dass im Falle der S-Inkommensurabilität die Optionen unvergleichbar und im Falle der L-Inkommensurabilität vergleichbar sind. Ich deute dies so, dass die Möglichkeit der Substitution entscheidend für Vergleichbarkeit ist. Konkret heisst das Folgendes: Im Falle der S-Inkommensura- 
bilität werden (i) Arbeit und Freizeit in unterschiedlichen Berufen unterschiedlich realisiert (z. B. 80-Prozent- versus 100-Prozent-Stelle), (ii) können Arbeit und Freizeit nicht substituiert werden. Deshalb sind (iii) die beiden Berufe unvergleichbar. Dabei wird vorausgesetzt, dass keine L-Inkommensurabilität gegeben ist, also Arbeit der Freizeit nicht lexikographisch vorgezogen wird (oder umgekehrt). Falls nämlich Arbeit Freizeit immer vorgezogen würde (oder umgekehrt), wären die beiden Standards ceteris paribus vergleichbar. Anders formuliert: Eine lexikographische Ordnung der Standards macht ihre Substituierbarkeit (i) nicht nur unmöglich, sondern (ii) auch überflüssig. Das heisst, dass Substituierbarkeit keine notwendige Bedingung für Vergleichbarkeit mehr darstellen würde (D’Agostino 2003: 9). Einige Philosophen interpretieren sogenannte konstitutive Inkommensurabilität denn auch als lexikographische Ordnung, was uns zu einer weiteren Auffassung von Inkommensurabilität führt.

Konstitutive Inkommensurabilität meint, dass es konstitutiv für gewisse Werte und Güter ist, dass wir sie nicht vergleichen können/sollen. Dabei lassen sich zwei Deutungen unterscheiden. Erstens kann konstitutive Inkommensurabilität wie gesagt als Priorisierung interpretiert werden. Zwei Werte sind dementsprechend genau dann inkommensurabel, wenn die Instanziierung des einen Werts immer besser als die Instanziierung des anderen Werts ist (vgl. Dworkin 1977). Das Recht auf Nahrung (zum Beispiel genügend Reis zu haben) überwiegt beispielsweise stets den Wert des ökonomischen Handelns (zum Beispiel Reis zu kaufen und zu verkaufen). Man kann dies auch so verstehen, dass gewisse Werte einen höheren Status haben als andere. Anderson spricht in diesem Zusammenhang von 'hierarchischer Inkommensurabilität' (Anderson 1997: 104). Zweitens kann konstitutive Inkommensurabilität aber auch so interpretiert werden, dass durch das Vergleichen gewisse Dinge unterminiert werden. Raz argumentiert, dass jemand, der Freundschaft und Geld als vergleichbar ansieht, überhaupt nicht in der Lage ist, mit einer anderen Person eine Freundschaft einzugehen (Raz 1986). Er kann so verstanden werden, dass es (i) charakteristisch für gewisse Dinge wie Freundschaft ist, dass wir glauben, dass sie nicht mit anderen Dingen vergleichbar sind, und dass (ii) deshalb Geld und Freundschaft konstitutiv unvergleichbar sind. In diese Richtung argumentiert auch Lukes. Gemäss 
Lukes meint konstitutive Inkommensurabilität, dass man bei der Verfolgung gewisser Ziele sogenannte Bindungen (commitments) tätigen muss, welche die Erreichung anderer Ziele verunmöglicht. Freundschaft verlangt als Bekenntnis beispielsweise den Glauben an die Nicht-Vergleichbarkeit von Freundschaft und Geld (Lukes 1997). Der Streit zwischen den beiden Deutungen besteht dann in der Auseinandersetzung mit der Frage, ob konstitutive Inkommensurabilität auf Priorisierung zurückgeführt werden kann. Schaber 1994 und Chang 2001 verstehen erstere beispielsweise nicht wie Raz oder Lukes als Nicht-Vergleichbarkeit, sondern wie Anderson als lexikographische Priorisierung. Ähnlich sieht es auch Betzler. Gemäss Betzler sind zwischenmenschliche Beziehungen als intrinsische Werte, Geld jedoch nur als instrumenteller Wert zu charakterisieren, wobei die Ablehnung, Menschen zu verkaufen, die Vergleichbarkeit der beiden Dinge voraussetzt (Betzler 1999: 308).

Nun gibt es allerdings noch weitere Definitionen. Inkommensurabilität kann beispielsweise auch als Inkompatibilität abstrakter Werte verstanden werden (Berlin 1969: 49). Damit ist gemeint, dass allgemeine Werte wie Wissen und Glück manchmal unverträglich sind. Dies wiederum kann so verstanden werden, dass die Erreichung des einen Werts zuweilen mit dem Verlust des anderen Werts einhergeht. Wenn man weiss, dass es 2,5 Planeten bräuchte, würden alle Menschen so leben wie wir in der Schweiz, stimmt dies einen vielleicht nachdenklich, und also führt mehr Wissen in diesem Fall zu weniger Glück. Allenfalls wird Unvergleichbarkeit auch so verstanden, dass zwei moralische Normen weder 'stärker' noch gleich 'stark' sind: '(...), moral requirements are incomparable just in case neither is stronger than, weaker than or equal in strength to the other.' (SinnottArmstrong 1985: 324).

Zusammenfassend kann festgehalten werden, dass sich die bestehenden Sichtweisen von Unvergleichbarkeit und Inkommensurabilität wie folgt voneinander unterscheiden:

Erstens: Unvergleichbarkeit und Inkommensurabilität werden manchmal synonym und manchmal abweichend definiert.

Zweitens: Die Relata $x$ und $y$ sind unterschiedlich. Das heisst, dass der Gegenstand der Unvergleichbarkeit oder Inkommensurabilität variiert. Manchmal sind damit konkrete und realisierbare 
Optionen, manchmal Gegenstände ${ }^{11}$, manchmal sogar Personen und manchmal abstrakte Werte gemeint.

Drittens: Zusammenhängend mit dem vorherigen Punkt werden Unvergleichbarkeit und Inkommensurabilität manchmal in einem Entscheidungskontext diskutiert und manchmal nicht. Viele Beispiele von Unvergleichbarkeit und Inkommensurabilität sind so gewählt, dass eine Akteurin zwischen Optionen, die nicht gleichzeitig realisiert werden können, entscheiden muss. ${ }^{12}$ Dies ist jedoch nicht immer der Fall. Ein Beispiel stellen Mozart und Michelangelo dar, die hinsichtlich Kreativität verglichen oder nicht verglichen werden können (vgl. Chang 2002a). Und auch die Frage, ob zwei Kathedralen inkommensurabel sind, hat nicht unbedingt etwas mit einer Entscheidung zu tun (vgl. Broome 1997). ${ }^{13}$

Viertens: Das Konzept besser als, welches in den meisten Definitionen vorkommt, wird oft nicht weiter erläutert, manchmal als Präferenz, manchmal als Präferenz, die man haben soll oder darf, und manchmal als Relation zwischen Werten verstanden.

Fünftens: Es besteht keine Einigkeit darüber, wie viele Vergleichsrelationen es gibt. Die meisten Philosophinnen und Philosophen gehen davon aus, dass es drei Vergleichsrelationen gibt. Gemäss dieser Ansicht sagt uns die Trichotomie-These, wann zwei Dinge als vergleichbar betrachtet werden können. Unvergleichbarkeit kann dementsprechend als Negation der Trichotomie-These definiert werden. Diese Auslegung wird aber von all denjenigen Philosophinnen und Philosophen abgelehnt, welche die Meinung vertreten, dass es mehr als drei Vergleichsrelationen gibt respektive geben kann.

Sechstens: Es gibt alternative Definitionen von Inkommensurabilität, wie zum Beispiel, dass abstrakte Werte inkompatibel sind.

Man kann auch sagen, dass sich die bestehenden Definitionen von Unvergleichbarkeit und Inkommensurabilität (i) in der Anzahl der darin vorkommenden Vergleichsrelationen, (ii) in der Bedeutung der Vergleichsrelationen, (iii) in den Relata der Vergleichbarkeit und

11 Diese werden oft 'items' oder 'bearer of values' genannt und können in den meisten Fällen als potenzielle Wahlobjekte interpretiert werden (vgl. etwa Chang 2009).

12 Siehe etwa Raz 1986, Gert 2004 oder Chang 2002a.

13 Man könnte die Beispiele natürlich so modifizieren, dass es um Entscheidungen geht: Welche inkommensurable Kathedrale zeige ich der Schwiegermutter? 
(iv) im Kontext, in welchem Unvergleichbarkeit diskutiert wird, unterscheiden.

Bedeutet dies, dass die bestehenden Sichtweisen überhaupt keine Gemeinsamkeit aufweisen?

Eine Übereinstimmung könnte darin bestehen, dass es stets um genau eine Person geht, die Entscheidungen treffen muss und Werte oder Optionen vergleichen respektive nicht vergleichen kann. Es handelte also beispielsweise nicht davon, was Wert hat und was nicht, wenn eine Person $x$ wertvoll und eine andere Person non- $x$ beziehungsweise $y$, welches non-x impliziert, wertvoll findet (der bürgerliche Politiker meint eher Freiheit und der linke Politiker eher distributive Gerechtigkeit). In diesem Zusammenhang existieren aber Gegenbeispiele. D’Agostino studiert Inkommensurabilität in seinem Buch Incommensurability and Commensuration sowohl auf einer intrapersonalen (individuelle Entscheidungen) als auch auf einer interpersonalen Ebene (kollektive Entscheidungen). Sinnott-Armstrong und Richardson diskutieren Unvergleichbarkeit im Zusammenhang mit moralischen Dilemmata. ${ }^{14}$ Auch Adlers Definition von Inkommensurabilität - 'Options are incommensurable, simpliciter, for an agent, if in light of the total of normative considerations the agent has reason not to use any scaling procedure in choosing between those options.' - ist ausschliesslich für einen ethischen Kontext gedacht (Adler 1998: 1380). Dies geht dann insofern über eine einzelne Person hinaus, als der Anspruch allgemeiner ist. Und nicht zuletzt kann auch der Ansatz der lexikographischen Priorisierung bei einigen Philosophen so verstanden werden, dass sie für mehr als eine Person gilt. ${ }^{15}$ Mit der These, dass gewisse Werte wie genügend Nahrung zu haben einen höheren Status als andere aufweisen, scheint nämlich ein universeller Anspruch einherzugehen (es gilt für alle). Eine solche Interpretation lexikographischer Priorisierung erinnert an deontologische Ansätze in der Ethik, die gewissen Rechten und Pflichten uneingeschränkte Geltung zuschreiben. ${ }^{16}$

14 Vgl. Sinnott-Armstrong 1985, Richardson 1994: 115, 116, 117.

15 Ich denke z. B. an Dworkin 1977 und Anderson 1997.

16 Lexikographische Vergleichbarkeit wird dann so verstanden, dass man nicht zu vergleichen braucht (im Sinne von Abwägen), weil das eine stets dominiert, z. B das Recht auf Nahrung uneingeschränkt gilt. Innerhalb bestimmter deontologischer Ansätze würde bei 
Eine andere Gemeinsamkeit könnte darin bestehen, dass der Begriff der Vergleichbarkeit stets als dreistelliges Prädikat verwendet wird. Ein Vergleich setzt stets eine Basis für das Vergleichen voraus. Das würde heissen, davon auszugehen, dass zwei Optionen/Gegenstände/Werte immer bezüglich etwas verglichen werden. Chang nennt dieses Etwas, welches man auch Entscheidungskriterium nennen könnte, Deckwert (covering consideration) (Chang 2002a: 666). Wenn eine Akteurin das Studium der Ökonomie und dasjenige der Rechtswissenschaft vergleicht, stellt 'gutes Studium' den Deckwert dar, hinsichtlich dessen die beiden Studiengänge verglichen werden. Chang würde sagen, dass ihre Definition von Unvergleichbarkeit im Sammelband Incommensurability, Incomparability and Practical Reason - 'Two items are incomparable if nopositive value relation holds between them.' (Chang 1997: 4) - elliptisch ist für folgende Definition: 'Two items are incomparable if it is false that any positive bias, binary value relations holds between them with respect to a covering consideration.' (Chang 2014: 4). Die notwendige Bedingung würde in diesem Fall darin bestehen, dass es nicht möglich ist, zwei Optionen simpliciter zu vergleichen. Auch in diesem Fall existieren allerdings Gegenbeispiele. Schaber etwa geht wie die Mehrheit der Philosophinnen und Philosophen davon aus, dass die Trichotomie-These richtig ist und Optionswerte mit den Ausdrücken besser, schlechter und gleich gut aufeinander bezogen werden. Um dies zu tun, so Schaber, brauchen wir allerdings keinen zentralen Vergleichswert. ${ }^{17}$ Schaber hat Recht. Ein Deckwert kann die unterschiedlichen Definitionen deshalb nicht einen, weil zum Beispiel eine Priorisierung, aber auch eine Substitution zweier Standards (Arbeit versus Freizeit) keinen Deckwert voraussetzen.

der Formulierung jedoch eine stärkere Modalität verwendet werden, nämlich, dass man nicht abwägen soll. Nur extreme deontologische Ansätze gehen allerdings davon aus, dass Pflichten keine Abwägungen erlauben. Moderate deontologische Positionen lassen zu, dass andere Dinge Pflichten übertrumpfen können (vgl. Chang 2013a: 2594).

17 Inwiefern Schabers Begründung überzeugend ist - ein Deckwert wäre nur dann erforderlich, wenn kardinale Wertgrössen angestrebt würden (Schaber 1994: 154) -, soll an dieser Stelle nicht diskutiert werden. Schaber vertritt wie viele andere Philosophen eine präferenzbasierte Sichtweise von Unvergleichbarkeit. Im nächsten Kapitel wird ein Lösungsvorschlag präsentiert auf die Frage, ob eine solche Version von Unvergleichbarkeit ein drei oder zweistelliges Konzept darstellt. 
Eine weitere potenzielle Gemeinsamkeit könnte darin bestehen, dass Unvergleichbarkeit und Inkommensurabilität als Relation oder vielmehr als Abwesenheit einer Relation zwischen Optionen, Werten, Personen oder Gegenständen verstanden werden müssen. Ein solcher Begriff wäre allerdings so allgemein, es würden so viele Dinge darunter fallen, dass er nicht mehr aussagekräftig wäre.

In der Konsequenz heisst dies, dass die bestehenden Definitionen tatsächlich keine einschlägige Gemeinsamkeit besitzen.

Angesichts dieser verschiedenen Auffassungen von Unvergleichbarkeit und Inkommensurabilität stellt sich die Frage, wie damit umgegangen werden soll. Eine Möglichkeit könnte darin bestehen, aufgrund einer klassischen Begriffsanalyse zu einer richtigen Definition zu gelangen. Weshalb dies keine geeignete Strategie darstellt, wird im nächsten Teil erläutert.

\subsection{Weshalb keine Begriffsanalyse?}

Erstens argumentiere ich, dass eine klassische Begriffsanalyse nicht möglich ist. Zweitens erläutere ich, weshalb die Suche nach Familienähnlichkeiten nicht zielführend ist.

Fragen der Art 'Was ist Unvergleichbarkeit?' beziehungsweise 'Was ist X?' besitzen eine lange philosophische Tradition. Das Instrumentarium der Begriffsanalyse wurde und wird immer noch als Methodeverwendet, um Fragen dieses Typus beantworten zu können. Was muss man sich unter einer klassischen Begriffsanalyse vorstellen, und weshalb bringt uns dies nicht weiter? Eine klassische Begriffsanalyse geht von einer klassischen Definitionslehre aus. Diese Möglichkeit der Begriffsanalyse verwendet etwa Sokrates in Platons Dialogen. ${ }^{18}$ Platon denkt dabei zusätzlich an eine sogenannte reale Definition. Gemäss einer realen Definition soll die Sache an sich definiert werden - in Abgrenzung zu unserem Verständnis der Sache. Platon würde vermutlich davon ausgehen, dass ein bestimmtes Phänomen der Unvergleichbarkeit existiert, unabhängig davon, welches Verständnis dieses Begriffs wir haben. Das heisst, dass mit einer Definition von Unvergleichbar- 
keit nach Platon das Wesen von Unvergleichbarkeit freigelegt würde. Ich denke, dass die Unterscheidung zwischen der Sache selbst und unserem Verständnis davon allerdings unbrauchbar ist (es geht immer auch um unser Verständnis). Ich würde es eher so verstehen, dass wir alle, egal ob Bäckerin, Journalistin oder Philosophin, ein implizites Wissen über das Phänomen der Unvergleichbarkeit und der Inkommensurabilität besitzen. Gemäss diesem Gedanken müssen Begriffsdefinitionen realen oder hypothetischen Situationen ausgesetzt werden, damit ihre Plausibilität überprüft werden kann. Das heisst nichts anderes, als dass die verschiedenen Definitionen, wie diejenige von Chang, mit Gegenbeispielen konfrontiert werden sollten, in denen die Definition versagt. Im Falle von Chang würde dies eine Situation darstellen, in denen zwei Optionen weder kardinal verglichen werden können noch inkommensurabel sind. Das Ziel besteht hierbei letztlich darin, eine Definition zu finden, die gegen Gegenbeispiele immun ist. Ein Gegenbeispiel kann dabei auf zwei Arten formuliert werden: Entweder ist eine in der Definition erwähnte Bedingung nicht notwendig, oder sie ist nicht hinreichend. Im ersten Fall gibt es ein Ding, das unter den Begriff fällt, das aber die vorgeschlagene Bedingung nicht erfüllt. Im zweiten Fall gibt es ein Ding, das die vorgeschlagene Bedingung erfüllt, das aber nicht unter den Begriff fällt. ${ }^{19}$ Sind keine Gegenbeispiele dieser Art mehr möglich, ist das Ziel erreicht. ${ }^{20}$ Das heisst nichts anderes, als dass die allgemeine Bedingung dafür aufgestellt worden ist, in welchem Fall der Begriff zutrifft. ${ }^{21}$ Tisch genau dann, wenn $X$ ein Möbelstück ist, an dem gegessen wird.' In diesem Fall kann wie folgt argumentiert werden, dass die in der Definition erwähnte Bedingung weder hinreichend noch notwendig ist: Ein Nachttisch ist ein Tisch, jedoch wird an einem Nachttisch nicht gegessen. Das heisst, dass es ein Ding gibt, welches unter den Begriff fällt, das aber die vorgeschlagene Bedingung nicht erfüllt. Ergo ist die Bedingung nicht notwendig. An einer Bar hingegen kann gegessen werden, eine Bar ist jedoch kein Tisch. Das heisst, dass es ein Ding gibt, welches die vorgeschlagene Bedingung erfüllt, das aber nicht unter den Begriff fällt. Ergo ist die Bedingung nicht hinreichend (Pfister 2013: 71, 72).

20 Etliche Schwierigkeiten werden an dieser Stelle ausgeklammert. Es müssen zusätzliche Bedingungen erfüllt sein, damit das Ziel tatsächlich erreicht ist. Es stellt keine hinreichende Bedingung einer solchen Definition dar, dass keine Gegenbeispiele formuliert werden können. Darauf kann nicht eingegangen werden.

21 Mit einer Bedingung ist im einfachsten Fall eine einzige und im Normalfall eine Summe von einzeln notwendigen und zusammengenommen hinreichenden Bedingungen gemeint. 
Diese Art von Begriffsanalyse kann deshalb nicht angewendet werden, weil insbesondere Inkommensurabilität, aber auch Unvergleichbarkeit sogenannte technische Begriffe sind. Die Überlegungen im ersten Teil des Kapitels legen den Schluss nahe, dass keine paradigmatischen Fälle existieren, in denen kompetente Sprecherinnen übereinstimmen, dass Inkommensurabilität vorliegt oder nicht. Dies kann so verstanden werden, dass es keine typischen Situationen der Inkommensurabilität gibt. Im Falle von normalsprachlichen Ausdrücken verhält sich dies anders. Manchmal ist etwas eindeutig ein Tisch oder eindeutig eine Form von Nicht-Wissen. Oder manchmal ist eine Person eindeutig nicht autonom, beispielsweise dann nicht, wenn sie immer und unhinterfragt dasjenige tut, was die Eltern meinen. Je nach Grad der Vagheit (es ist unklar, ob ein Gegenstand unter den Begriff fällt) existieren dann mehr oder weniger Grenzfälle. Der Terminus des Verkehrsschilds beispielsweise besitzt sehr klare Anwendungsbedingungen. Selbst bei vagen Prädikaten, und das betrifft beinahe alle umgangssprachlichen Begriffe, existieren jedoch viele eindeutige Situationen, in denen ein Begriff angewendet werden kann oder nicht.

In diesem Zusammenhang können zwei Einwände vorgebracht werden. Der eine besagt, dass Unvergleichbarkeit einen normalsprachlichen Terminus darstellt. Ergo ist es falsch, dass keine paradigmatische Fälle existieren. Beispielsweise stellt es einen typischen Fall von Unvergleichbarkeit dar, dass Lionel Messi und Manuel Neuer unvergleichbar sind. Man kann nicht sagen, welcher besser Fussball spielt, weil Neuer ein Torwart und Messi ein Feldspieler ist. Oder es stellt beispielsweise einen typischen Fall dar, dass Zahlen und Äpfel nicht miteinander verglichen werden können. Ich denke jedoch, dass die im ersten Teil erläuterten Sichtweisen von Unvergleichbarkeit technische Ansätze sind. Mir scheint, dass dies eine nicht weiter zu begründende Beobachtung ist. Dass dabei 'lose’ Beziehungen zwischen bestimmten alltäglichen Redeweisen oder Meinungen und den erläuterten philosophischen Konzepten bestehen, soll nicht ausgeschlossen werden. Man kann argumentieren, dass die Leute denken, Zahlen und Früchte seien unvergleichbar, weil unterschiedliche $\mathrm{Ka}$ tegorien involviert sind und es zur Definition von Unvergleichbarkeit passt, dass keine gemeinsame Basis für den Vergleich (Deckwert) existiert. Der andere Einwand lautet, dass es auch im Falle technischer 
Termini eindeutige Situationen gibt, in denen ein Begriff Anwendung findet. Man könnte sich beispielsweise auf den Standpunkt stellen, dass 'analytischer Philosoph' ein technischer Begriff ist und trotzdem eindeutige Situationen existieren: Russell ist einer und Heidegger nicht. Ich denke, dass man dies zugestehen kann, es sich im Falle von Unvergleichbarkeit und Inkommensurabilität aber nicht so verhält. Anders als beim analytischen Philosophen können keine Eigenschaften wie 'sprachliche Klarheit' oder 'Argumente nennen' angefügt werden, weshalb in dieser Situation Inkommensurabilität vorliegen soll und in jener nicht. Es gibt keine sinnvollen Argumente, weshalb die Inkompatibilität abstrakter Werte im Gegensatz zu kardinaler Unvergleichbarkeit als Inkommensurabilität bezeichnet werden sollte.

Eine klassische Begriffsanalyse stellt also deshalb keine sinnvolle Strategie dar, um zu einer richtigen Definition von Unvergleichbarkeit zu gelangen: Wir besitzen kein implizites Verständnis von Unvergleichbarkeit und Inkommensurabilität, das abgerufen werden könnte. Dafür werden die Begriffe der Inkommensurabilität und Unvergleichbarkeit ausserhalb akademischer Debatten schlicht zu wenig verwendet, um nicht zu sagen, gar nicht. Und falls wir mit den Begriffen dennoch bestimmte alltägliche Vorstellungen verbinden, ist fraglich, was dies für die Fülle an technischen Definitionen bringt. Dass die Begriffe der Unvergleichbarkeit und der Inkommensurabilität keine oder beinahe keine umgangssprachliche Verwendung aufweisen, scheint aber ein Vorteil zu sein. Einwände der Art 'In Kontext X wird der Begriff der Unvergleichbarkeit anders verwendet' zielen somit nämlich ins Leere. Entweder sind sie nicht möglich oder, falls doch, nicht relevant. Die technischen Ansätze referieren dann auf eine andere Idee, und dementsprechend würde aneinander vorbeigeredet werden. Inwiefern deshalb einerseits eine Begriffsexplikation angebracht ist, andererseits Unvergleichbarkeit trotzdem in einem bestimmten Sinn reale Aspekte aufweist (nicht in Platons Sinne), wird im letzten Teil des Kapitels gezeigt.

Eine andere Version der Begriffsanalyse besteht darin, Familienähnlichkeiten ausfindig zu machen. Was ist damit gemeint und weshalb ist auch diese Methode nicht zielführend?

Die Idee der Familienähnlichkeit geht auf Wittgenstein zurück. Wittgenstein lehnt die klassische Begriffsanalyse ab. Er argumentiert in den Philosophischen Untersuchungen anhand des Begriffs des Spiels, 
dass für umgangssprachliche Termini keine notwendigen Bedingungen angegeben werden können. Damit meint Wittgenstein, dass keine Eigenschaft existiert, die alle Spiele gemeinsam aufweisen. Ob es sich um Spiele oder Tische handelt, spielt keine Rolle. Vielmehr, so Wittgenstein, erkennt man bei umgangssprachlichen Termini Überlappungen, Ähnlichkeiten und kleine und grosse Unterschiede - er benutzt in diesem Zusammenhang die Metapher der Familienähnlichkeit (Wittgenstein 1984: 66). Ich würde dies in Anlehnung an Daly wie folgt verstehen: Wenn zwischen verschiedenen Dingen, $a$, $b, c \ldots$, die Beziehung der Familienähnlichkeit besteht, dann haben diese Dinge im folgenden Sinne nichts gemeinsam: Zwei Dinge, $a$ und $b$, gleichen sich auf eine Weise, in der sich zwei andere Dinge, $b$ und $c$, nicht gleichen müssen (Daly 2010: 219). In dieser Art Familienähnlichkeiten zu benennen, ist im Gegensatz zu einer klassischen Begriffsanalyse nicht zwingend zum Scheitern verurteilt. Man könnte argumentieren, dass es zwar kein Charakteristikum gibt, welches sämtliche Sichtweisen eint, die verschiedenen Ansätze aber gleichwohl Ähnlichkeiten aufweisen. Ein Beispiel: Die Situation, dass man keine Alternative präferiert und nicht indifferent ist, besitzt Ähnlichkeit damit, dass zwei Werte nicht besser/schlechter/gleich gut sind (es gibt drei Vergleichsrelationen). Die Situation, dass zwei Werte nicht besser/schlechter/gleich gut sind, besitzt Ähnlichkeit damit, dass zwischen zwei Gegenständen keine positive Wertrelation besteht (eine Wert- lässt sich nicht auf eine Präferenzrelation reduzieren). Das heisst, dass sich die erste und zweite Situation darin gleichen, dass von drei Vergleichsbeziehungen ausgegangen wird, während sich die zweite und dritte Situation darin nicht gleichen. Vielmehr sind sie insofern ähnlich, als eine Wertrelation etwas anderes als eine Präferenzrelation ist. Man könnte ein solches Projekt verfolgen und im besten Falle Ähnlichkeiten aufdecken. Die Beantwortung der Frage, was man damit gewonnen hätte, überlasse ich aber lieber jemandem, der ein solches Vorhaben realisieren möchte. Ich will nicht ausschliessen, dass die Suche nach Familienähnlichkeiten zu neuen Erkenntnissen führen kann. Analog kann man sich vorstellen, dass man mehr über das Wesen von Spielen erfährt, wenn Familienähnlichkeiten innerhalb der verschiedenen Spiele aufgedeckt werden. Hinsichtlich der Beantwortung folgender Fragen ist die Suche nach Familienähnlichkeiten 
allerdings nicht zielführend: Was muss präzise unter Unvergleichbarkeit verstanden werden? Worin besteht das Problem für die Rationalität? Wie soll mit Unvergleichbarkeit umgegangen werden?

Zusammenfassend kann festgehalten werden, dass weder eine klassische Begriffsanalyse noch die Suche nach Familienähnlichkeiten geeignete Methoden darstellen, um die vorliegende Ausgangslage es existieren sehr unterschiedliche Verwendungsweisen der beiden Begriffe - vereinfachen zu können. Mithilfe dieser Methoden gelingt es nicht, einzelne Definitionen von Unvergleichbarkeit und Inkommensurabilität als plausibel oder unplausibel auszuweisen. Kann man Unvergleichbarkeit und Inkommensurabilität dementsprechend so definieren, wie man will? Weshalb dies nicht der Fall ist, wird im letzten Teil des Kapitels erläutert.

\subsection{Begriffsexplikation, Stipulation und reale Elemente von Unvergleichbarkeit}

Erstens argumentiere ich, dass eine Begriffsexplikation einer stipulativen Definition vorgezogen werden sollte. Zweitens zeige ich, dass dies kompatibel damit ist, dass eine Definition von Unvergleichbarkeit reale Komponenten besitzt. Ich erläutere, was damit gemeint ist, inwiefern einige Begriffsbestimmungen dennoch stipulativ oder eher willkürlich sind und welche Konsequenzen sich daraus für den weiteren Verlauf des Buches ergeben.

Selbst wenn man die Definition von Unvergleichbarkeit stipulativ einführen würde, wäre man dabei nicht völlig frei. Eine stipulative Definition meint, dass festgelegt wird, wie ein Begriff in einem bestimmten Kontext verwendet wird. Mit einer festlegenden Definition legt man, wie der Name sagt, eine neue Bedeutung fest, unabhängig davon, welche Bedeutung der Begriff bereits hat. Dies kann so verstanden werden, dass ein Begriff dasjenige bedeutet, was man wählt (Robinson 1950: 59). Man definiert selber, was er bedeuten soll. Ich denke, dass dies allerdings nur theoretisch stimmt. Pragmatisch gesehen, zumindest im Falle philosophischer Fachtermini, sollte man sich auch bei einer stipulativen Definition an den bestehenden Begriffsbestimmungen orientieren. Zumindest dann, wenn es sich, wie 
im Falle von Unvergleichbarkeit und Inkommensurabilität, um etablierte philosophische Begriffe handelt. Einerseits würde eine abweichende Definition nämlich Verwirrung stiften, und andererseits stehen etablierte Begriffe in begrifflicher Relation zu anderen Begriffen, die dementsprechend ebenfalls neu definiert werden müssten (Glock 2008: 13). Eine begriffliche Relation wird dabei oft so verstanden, dass die Intensionen von Begriffen - das sind Merkmale, aufgrund derer etwas unter einen Begriff fällt - ein bestimmtes Verhältnis ihrer $E x$ tensionen festlegen - damit ist die Menge der Gegenstände gemeint, die unter den Begriff fallen (vgl. etwa Brun und Hirsch Hadorn 2009). Das heisst, dass durch eine abweichende Definition diese bestehenden Verhältnisse durcheinandergebracht würden. Ein Beispiel: Obwohl einige Philosophinnen und Philosophen die Begriffe der Inkommensurabilität und Unvergleichbarkeit synonym verwenden, gibt es viele, die differenzieren, etwa im folgenden Sinne: Zwei Optionen sind genau dann unvergleichbar, wenn sie nicht als besser, schlechter oder gleich gut bewertet werden können, während zwei Werte genau dann inkommensurabel sind, wenn sie sich nicht hinsichtlich eines Deckwerts vergleichen lassen. In diesem Fall stellt Inkommensurabilität keine hinreichende Bedingung für Unvergleichbarkeit dar. Es ist nämlich möglich, dass sich Werte wie ein besseres Verständnis wirtschaftlicher oder juristischer Zusammenhänge nicht hinsichtlich eines gemeinsamen Werts wie 'interessantes Studium' vergleichen lassen und eine Akteurin das eine Studium trotzdem als das bessere bewerten kann. Man kann sich vorstellen, dass der Akteurin primär gesellschaftlicher Status und nicht Erkenntnis wichtig und dieser in den beiden Studiengängen unterschiedlich hoch ist. Nehmen wir nun an, dass wir 'Unvergleichbarkeit' neu so definieren, dass sich zwei Dinge nicht numerisch vergleichen lassen. In diesem Fall würde 'Inkommensurabilität' neu eine hinreichende Bedingung für 'Unvergleichbarkeit' darstellen (wenn überhaupt kein Deckwert vorhanden ist, liegt auch kein numerischer vor).

Auf jeden Fall kann eine stipulative Definition nicht richtig oder falsch sein. Oder anders formuliert: Über die Richtigkeit einer stipulativen Definition kann nicht sinnvoll gestritten werden. Wenn ich zum Beispiel sage, dass zwei Optionen genau dann unvergleichbar sind, wenn $X$ der Fall ist, lege ich mich zwar darauf fest, dass ich den Begriff 
der Unvergleichbarkeit in dieser Arbeit stets auf diese Weise verwenden werde. In diesem Sinne beinhaltet auch eine stipulative Definition eine Verpflichtung. Aber sie kann weder korrekt noch unkorrekt sein. Über die Angemessenheit einer solchen Definition kann man aber, wie obige Überlegungen zeigen, sehr wohl verschiedener Meinung sein.

Ich denke jedoch, dass eine Begriffsexplikation, welche die im letzten Abschnitt genannten Eigenschaften einer Stipulation ebenfalls aufweist, einer rein stipulativen Einführung vorgezogen werden sollte. Der Grund dafür ist, dass in der philosophischen Diskussion ja bereits von Unvergleichbarkeit gesprochen wird (es muss kein neuer Begriff eingeführt werden), und ich im nächsten Kapitel versuche, diesen Begriff anhand einfacher und exakter Begriffe zu charakterisieren und in einen grösseren theoretischen Kontext einzubetten. Was meint hierbei eine Begriffsexplikation? Die sogenannte Begriffsexplikation geht u. a. auf Carnap zurück (Carnap 1959; vgl. hierzu auch Greimann 2007). Im Unterschied zu einer klassischen Definition handelt es sich bei einer Explikation in einem bestimmten Sinne um keine Identitätsbeziehung (obwohl im Resultat der Explikation eine Definition steht). Das sogenannte Explikat ist nämlich teilweise verschieden vom sogenannten Explikandum (obwohl Ähnlichkeit ein Adäquatheitskriterium einer Explikation ist). Das Explikat und das Explikandum sind nicht extensionsgleich (Brun und Hirsch Hadorn 2009: 183). Eine Explikation kann im Gegensatz zu einer realen Definition und gleich wie eine Stipulation nicht wahr oder falsch sein. Sie ist entweder angemessen oder unangemessen (Carnap 1959: 12). Ein Beispiel: Ein Mann ist (i) ein Lebewesen und besitzt (ii) einen $\mathrm{x}$ - und einen $\mathrm{y}$ Chromosomensatz. Ein Mann ist (i) ein Lebewesen und (ii) älter als 18 Jahre. In beiden Fällen wird das Explikandum (Mann) anhand eines verschiedenen Explikats erläutert. In diesem Zusammenhang wird meistens angenommen, dass bestimmte Adäquatheitsregeln erfüllt sein müssen wie beispielsweise, dass das Explikandum und das Explikat ähnliche Anwendungsbedingungen besitzen und das Explikat exakter und einfacher als das Explikandum ist.

Eine Begriffsexplikation ist dabei kompatibel damit, dass eine Definition von Unvergleichbarkeit reale Elemente beinhaltet. Bevor aber den Fragen nachgegangen wird, was mit real genau gemeint ist und welche Konsequenzen sich daraus für den weiteren Verlauf 
ergeben, soll kurz erläutert werden, welche Bestimmungen gleichwohl stipulativ oder eher willkürlich sind.

Erstens ist die Festlegung in einem gewissen Masse willkürlich, worin das Erkenntnisinteresse liegt; soll beispielsweise genauer auf Inkompatibilität, lexikographische Priorisierung oder kardinale Nicht-Vergleichbarkeit (alles verschiedene Verwendungsweisen von Inkommensurabilität) oder Unvergleichbarkeit eingegangen werden? Mich interessiert Unvergleichbarkeit und beispielsweise nicht lexikographische Priorisierung oder die kardinale Nicht-Vergleichbarkeit von Optionen. Der Grund dafür ist, dass Unvergleichbarkeit das Problem für die Rationalität generiert. Weil für uns Rationalität im Kontext von Entscheidungen relevant ist, werden die Relata dabei als Handlungsoptionen und nicht etwa als abstrakte Werte interpretiert. Ich werde in Kapitel 6 zeigen, inwiefern Unvergleichbarkeit ein Problem für die rationale Entscheidungsfindung darstellt. An dieser Stelle kann vereinfachend festgehalten werden, dass ein Akteur dann mit einer Schwierigkeit konfrontiert ist, wenn er die Optionen nicht als besser/schlechter/gleich gut bewerten kann. Wenn ein Akteur nicht angeben kann, welches Studium wertvoller ist, stellt dies in dem Sinne ein Problem dar, als er dann nicht weiss, wofür er sich entscheiden soll. Wenn ein Akteur gewisse Werte, die in den unterschiedlichen Studiengängen realisiert werden, nicht hinsichtlich eines allgemeineren Werts vergleichen kann, stellt dies nicht zwingend eine Schwierigkeit dar. Beispielsweise deshalb nicht, weil er eine Priorisierung der inkommensurablen Werte vornehmen kann (Interesse kommt vor gesellschaftlichem Status).

Zweitens ist die Namensgebung willkürlich. Wenn man sagt, dass die Essenz oder das Wesen von Unvergleichbarkeit darin besteht, dass zwei Optionen weder nicht besser oder schlechter als noch gleich gut wie die jeweilig andere Option sind, kann dies wie folgt verstanden werden: Es gibt Situationen, in denen zwei Optionen weder besser oder schlechter als noch gleich gut wie die jeweilig andere Option sind (Deskription), und ich nenne dies Unvergleichbarkeit (Namensgebung). Man könnte es aber auch anders nennen. Die Deskription stellt wohlgemerkt eine reale Komponente dar. Damit kommen wir zur Frage, was real bedeutet.

Vereinfachend gesagt, meine ich mit real zwei Dinge: Gibt es Unvergleichbarkeit? Falls ja, weshalb gibt es Unvergleichbarkeit? 
Abstrakter formuliert bezieht sich der reale Teil der Definition von Unvergleichbarkeit erstens darauf, ob die Deskription wahr ist (in Anlehnung an Robinson 1950): Entweder gibt es Situationen, in denen die Optionen nicht als besser/schlechter/gleich gut bewertet werden können, oder es gibt sie nicht. Die Auseinandersetzung muss hierbei darin bestehen festzustellen, ob die Deskription wahr oder falsch ist. Ich werde argumentieren, dass die Deskription wahr ist. Es ist dieses bestimmte Phänomen, das untersucht wird.

Zweitens hat der reale Teil damit zu tun, unter welchen Bedingungen das Phänomen auftritt. Der philosophische Streit besteht hierbei darin, welches diese Bedingungen sind. Oder etwas anders ausgedrückt, die philosophische Auseinandersetzung kann als Suche nach Bedingungen verstanden werden, unter denen Unvergleichbarkeit vorkommt (Optionen nicht besser/schlechter/gleich gut sind).

Für den weiteren Verlauf des Buches bedeutet dies Folgendes: Erstens muss die Deskription genauer ausbuchstabiert werden. Es fehlt beispielsweise eine Explikation, was ‘besser als' meint. Das Phänomen muss genauer bestimmt werden. In diesem Fall erreicht man durch das Explizieren wichtiger Begriffe wie 'besser als' ein besseres Verständnis des realen Phänomens. Im nächsten Kapitel wird deshalb eine Präferenzversion der Unvergleichbarkeit eingeführt und verteidigt, im übernächsten Kapitel weiter präzisiert und in verschiedene harte Fälle aufgeteilt. Zweitens zielen die folgenden Kapitel dementsprechend darauf ab, die Bedingungen herauszuarbeiten, unter welchen Unvergleichbarkeit vorkommt.

Ich denke, dass dieses Vorgehen beliebig auf einen anderen Begriff von Inkommensurabilität und Unvergleichbarkeit angewendet werde könnte. Ein Beispiel: Es gibt Situationen, in denen zwei Werte in einer lexikographischen Ordnung stehen (Deskription), und ich nenne dies Inkommensurabilität (Namensgebung). Kommt das vor und falls ja, weshalb? In diesem Fall würde ein anderes Phänomen untersucht.

Besteht die philosophische Auseinandersetzung aber nicht 'aus mehr'? Und wie steht es um das Verhältnis von Unvergleichbarkeit und Inkommensurabilität?

Die Antwort auf die erste Frage fällt positiv aus. Einerseits muss die Sichtweise, dass Handlungsoptionen möglicherweise auch dann verglichen werden können, wenn sie weder besser oder schlechter als 
noch gleich gut wie die jeweilig andere Option sind, ernst genommen und nicht per definitionem ausgeschlossen werden. Ich argumentiere in Kapitel 7 und 8, dass es gute Gründe gibt, von drei Vergleichsbeziehungen auszugehen. In dieser Hinsicht besteht zwischen den unterschiedlichen Definitionen von Unvergleichbarkeit eine substanzielle Differenz. Ob man von drei oder mehr Vergleichsrelationen ausgeht, sollte keine Stipulation darstellen. Andererseits sollte die philosophische Auseinandersetzung in unserem Fall nicht auf die Beschreibung eines realen Phänomens reduziert werden - deshalb nicht, weil damit die ganze normative Frage vergessen wird, wie mit Unvergleichbarkeit umgegangen werden soll. Davon handelt dann insbesondere das letzte Kapitel des Buches.

Aber wie lässt sich Unvergleichbarkeit klar von Inkommensurabilität abgrenzen? Die Begriffe werden nicht synonym verwendet. Dies stellt eine stipulative Festlegung dar, was nicht heisst, dass es dafür keine Gründe gibt. Ein Argument besteht darin, dass die Mehrzahl der Philosophinnen und Philosophen (oft in Anlehnung an Chang) zwischen den Begriffen differenziert. Wenn wir dieser Tradition folgen, werden begriffliche Relationen nicht durcheinandergebracht.

Inkommensurabilität wird dabei so verstanden, dass es keine Möglichkeit der Beurteilung hinsichtlich eines gemeinsamen Deckwerts wie 'gutes Studium' gibt. In der Literatur haben sich Chang und insbesondere Hsieh damit auseinandergesetzt, was unter einem Deckwert genauer zu verstehen ist. Nach Hsieh sind Deckwerte 'klumpig'. Damit meint er, dass wir die zu vergleichenden Dinge in Klassen einordnen (Hsieh 2005). Changs Vorschlag besteht darin, dass ein Deckwert einen Platzhalter für einen abstrakteren und oft namenlosen Wert darstellt (Chang 2004). Diese Vorschläge werden in Kapitel 9 eingeführt und diskutiert. Ich werde argumentieren, dass Inkommensurabilität in einem bestimmten Sinn ein Argument für Unvergleichbarkeit darstellen kann. Optionen sind manchmal unvergleichbar, weil bei der Entscheidungsfindung kein Deckwert herbeigezogen werden kann. Aber damit dies der Fall ist - dies wird in Kapitel 9 gezeigt -, müssen zusätzliche Bedingungen erfüllt sein. Andererseits - dafür wird insbesondere in Kapitel 3 argumentiert ist evaluatives Unwissen und nicht Inkommensurabilität das stärkste Argument für Unvergleichbarkeit. Zudem entwickle ich in Kapitel 7 
eine eigene Theorie von Deckwerten beziehungsweise vagen Entscheidungskriterien. Diese Theorie impliziert, dass sehr präzise $\mathrm{Ab}-$ wägungen für uns Menschen schlicht zu kompliziert sind.

An dieser Stelle können wir jedoch vereinfachend festhalten, dass in diesem Buch das Phänomen der Unvergleichbarkeit im Zentrum steht und Inkommensurabilität in dem Sinne eine Rolle spielt, dass sie als eine der möglichen Bedingungen diskutiert wird, unter denen Unvergleichbarkeit vorkommt.

\subsection{Zusammenfassung}

Im ersten Teil des Kapitels wurde argumentiert, dass die bestehenden Sichtweisen von Unvergleichbarkeit und Inkommensurabilität keine einschlägigen Gemeinsamkeiten besitzen und sich in wesentlichen Merkmalen voneinander unterscheiden. Dazu zählen 'Anzahl der Vergleichsrelationen' (zum Beispiel drei versus mehr als drei Vergleichsrelationen), 'Bedeutung der Vergleichsrelationen' (zum Beispiel Präferenz versus Präferenz, die man haben soll), 'Relata der Vergleichbarkeit' (zum Beispiel konkrete Optionen versus abstrakte Werte) und 'Kontext' (zum Beispiel individuelle Entscheidung versus universeller Anspruch).

Im zweiten Teil des Kapitels wurde dargelegt, dass eine klassische Begriffsanalyse keine geeignete Methode darstellt, um die im ersten Teil systematisch dargestellten Sichtweisen auf einen gemeinsamen Nenner zu bringen.

Im dritten Teil des Kapitels wurde empfohlen, nicht den falschen Schluss zu ziehen, dass man Unvergleichbarkeit so definieren kann, wie man will. Ich zeigte, inwiefern eine Begriffsexplikation relevant ist und in welchem Sinne Unvergleichbarkeit reale Komponenten besitzt. Die Fragen, ob es Unvergleichbarkeit gibt oder nicht und unter welchen Bedingungen sie auftritt, stellen reale Komponenten dar, wobei sich die zweite Frage natürlich nur stellt, wenn die erste positiv beantwortet wird.

Nach diesen begrifflichen Betrachtungen geht es im nächsten Kapitel nun um den Versuch, eine präferenzbasierte Theorie von Unvergleichbarkeit zu entwickeln. 



\section{Kapitel 2}

Eine Präferenz-

version von

Unvergleich-

barkeit 
Wie im letzten Kapitel erläutert worden ist, gehen viele Philosophinnen und Philosophen von einer präferenzbasierten Definition von Unvergleichbarkeit aus:

Unvergleichbarkeit Zwei Optionen sind genau dann unvergleichbar, wenn eine Akteurin (i) keine Präferenz zugunsten der einen Option besitzt und (ii) auch nicht indifferent beziehungsweise gleichgültig zwischen den zur Wahl stehenden Alternativen ist.

Der Gegenstand dieses Kapitels besteht darin, eine Deutung eines solchen Ansatzes zu entwickeln. Wenn man eine präferenzbasierte Theorie von Unvergleichbarkeit vertritt, muss geklärt werden, (i) welcher Begriff einer Präferenz im Zusammenhang mit Unvergleichbarkeit von Bedeutung und (ii) welche Theorie des praktischen Nachdenkens dabei relevant ist. Das Kapitel besteht aus drei Teilen:

Im ersten Teil geht es um den Begriff der Präferenz. Dieser wird definiert. Innerhalb der Unvergleichbarkeits- und Inkommensurabilitätsdebatte ist dies bislang nur unzureichend geschehen. Der Begriff der Präferenz wird oft verwendet, seine Bedeutung aber nur selten explizit erklärt. Auch Ökonominnen und andere Sozialwissenschaftlerinnen, die diesen Terminus in formalen Entscheidungsmodellen verwenden, sagen jeweils nur sehr wenig zum Begriff selbst (vgl. Hausman 2012). Mit dem Ausdruck der Präferenz werden allerdings implizit unterschiedliche Ideen verbunden. Mein Ziel besteht darin zu zeigen, welche Deutung im Falle von Unvergleichbarkeit angebracht ist. Die These lautet, dass eine Präferenz als Evaluation verstanden werden sollte, die klärt, welche Option alles in allem besser ist.

Im zweiten Teil wird gezeigt, dass es um die sogenannte Konstruktion einer Präferenz geht und sich der Gegenstand des praktischen Überlegens auf den Inhalt der Präferenz richtet. Ich argumentiere dafür, dass hierbei Gründe eine Rolle spielen, genauer, dass die vorgeschlagene Theorie von Unvergleichbarkeit nicht mit einem rein instrumentellen Verständnis des praktischen Nachdenkens kompatibel ist.

Im dritten Teil wird die Präferenzversion gegen potenzielle Einwände verteidigt, wie zum Beispiel, dass Unvergleichbarkeit objektiv (unabhängig vom Akteur) und nicht subjektiv (abhängig vom Akteur) verstanden werden sollte. 


\subsection{Welcher Begriff der Präferenz ist im Zusammenhang mit Unvergleichbarkeit relevant?}

Eine Präferenzversion von Unvergleichbarkeit knüpft an der häufigsten Definition des Begriffs an, welche besagt, dass zwei Optionen, $x$ und $y$, genau dann unvergleichbar sind, wenn $x$ weder besser noch schlechter als noch gleich gut wie $y$ ist. Das charakteristische Merkmal einer präferenzbasierten Sichtweise von Unvergleichbarkeit besteht darin, dass 'besser als' beziehungsweise 'nicht besser als' meint, dass ein Akteur eine Option gegenüber einer anderen präferiert beziehungsweise nicht präferiert.

Wie jedoch soll in diesem Zusammenhang der Begriff der Präferenz verstanden werden? Negativ, also abgrenzend betrachtet, darf eine Präferenz nicht wie folgt interpretiert werden:

Erstens: Eine Präferenz meint nicht zwingend die Maximierung der eigenen Interessen. Präferenz wird nicht mit Eigeninteresse gleichgesetzt. Eine Präferenz muss in dieser Hinsicht als weiter Begriff verstanden werden. Präferenzen haben nicht unbedingt mit Egoismus oder Bedürfnisbefriedigung zu tun. Man kann die Zugreise nach Berlin der Reise mit dem Flugzeug vorziehen, auch wenn man lieber fliegen möchte (wenn man nur auf sich schauen würde). Das heisst, dass eine Präferenz zum Beispiel auch moralische, ästhetische, politische oder ökonomische Erwägungen beinhalten kann.

Zweitens: Eine Präferenz ist keine Frage des blossen Geschmacks. Über Geschmack lässt sich bekanntlich nicht sinnvoll streiten. Präferenzen hingegen können hinterfragt und von anderen kritisiert werden. Der Grund dafür ist, dass auf eine Präferenz 'willentlich' - mittels Abwägung von Gründen - Einfluss genommen werden kann. Darauf wird im zweiten Teil des Kapitels genauer eingegangen. Man könnte einwenden, dass Unvergleichbarkeit dementsprechend anhand des Begriffs der Absicht definiert werden sollte. Der Grund ist, dass auch eine Absicht auf deliberative Weise gebildet werden kann. Ich denke jedoch, dass die Charakterisierung über Präferenzen mehr Sinn ergibt. Einerseits ist dies deshalb der Fall, weil es bei Unvergleichbarkeit darum geht, in welchem Verhältnis Handlungsoptionen zueinander stehen. Eine Präferenz, die im Gegensatz zu einer Absicht eine Relation darstellt, wird diesem Umstand gerecht (ich komme gleich darauf 
zurück). Andererseits ist es denkbar, dass eine Akteurin $x$ beabsichtigt, obwohl $x$ und $y$ unvergleichbar sind. In Anlehnung an Anscombe formuliert, denkt die Akteurin dann 'Ich werde Ökonomie studieren' (vgl. Anscombe 1978: 11). Gleichzeitig ist sie jedoch nicht der Ansicht, dass es das bessere Studium als dasjenige der Rechtswissenschaft ist. Die Akteurin beabsichtigt $x$, obwohl sie keine Präferenz von $x$ gegenüber $y$ besitzt. In der Tendenz fällt es uns sicherlich schwer, eine Absicht für eine unvergleichbare Option zu bilden, im Sinne einer zukunftsgerichteten Einstellung (man beabsichtigt, zu einem späteren Zeitpunkt $x$ zu tun). Es ist schwierig, weil man sich nicht eindeutig zu ihr bekennen kann. Eine Absicht ändert die Zukunft (Scanlon 2008: 13), und man muss in gewisser Hinsicht 'hinter der Änderung stehen'. In diesem Sinne eine Absicht zu formulieren, die bei aller Stabilität ${ }^{1}$ widerrufen werden kann (Bratman 1987: 16), scheint im Falle von Unvergleichbarkeit dennoch möglich. Und ebenso kann man absichtlich handeln (man tut $x$ absichtlich). Absichtliche Handlungen sind gemäss Anscombe Handlungen, in denen die Frage 'Warum?' eine Rolle spielt (Anscombe 1978: 23). Ein Akteur kann als Antwort auf diese Frage Gründe nennen ('Das Studium der Ökonomie ist interessant') und in Anscombes Sinne absichtlich handeln, auch wenn die Optionen unvergleichbar sind.

Drittens: Eine Präferenz muss sich nicht zwingend in einer Zahlungsbereitschaft widerspiegeln. In der Ökonomie wird Zahlungsbereitschaft (willingness-to-pay) manchmal als Mass verwendet, um Präferenzen zu messen (vgl. etwa Lichtenstein und Slovic 1971 und Lindman 1971 für unterschiedliche Messmethoden). Gemäss diesem Ansatz präferiert der Akteur $x$ gegenüber $y$, wenn er für $x$ mehr bezahlen würde als für $y$. Man kann aber eine Präferenz von $x$ gegenüber $y$ besitzen, auch wenn es nicht möglich ist, dies in Geldeinheiten auszudrücken. Viele wichtige Dinge entziehen sich einer rein ökonomischen Angebot-Nachfrage-Logik, zum Beispiel kann man nicht Geld investieren, um (richtige) Freunde zu gewinnen. Eine Gleichsetzung mit Zahlungsbereitschaft würde eine zu starke ökonomische Reduktion darstellen.

Viertens: Eine Präferenz wird nicht über eine Wahl definiert. Weshalb nicht? Es stellt die simpelste Möglichkeit dar, Unvergleich- 
barkeit auszuschliessen. Die gewählte Option ist dann per definitionem die beste. Ergo gibt es keine unvergleichbaren Optionen, die weder besser noch schlechter sind. Diese Strategie kann jedoch nicht überzeugen. Ich werde im übernächsten Kapitel darlegen, weshalb eine bestimmte Interpretation der sogenannten Revealed-preferenceTheorie gegen Unvergleichbarkeit spricht, und zeigen, weshalb dieses Argument unplausibel ist.

Positiv betrachtet, lässt sich eine Präferenz wie folgt charakterisieren: Eine Präferenz kann als Relation zwischen Gegenständen (lieber Kaffee als Tee), zwischen Sachverhalten (der Zustand, in dem man Kaffee trinkt, ist besser als der Zustand, in dem man Tee trinkt) oder zwischen Handlungsoptionen (lieber eine Tasse Kaffee als eine Tasse Tee trinken) verstanden werden (vgl. hierzu etwa De Jonge 2012: 135).

In unserem Zusammenhang meint eine Präferenz eine Relation zwischen Handlungsoptionen. Ein Akteur kann eine Präferenz haben, dass Schweden und nicht Finnland Eishockeyweltmeister wird. Weil er aber darauf keinen Einfluss nehmen kann, ist die Präferenz nicht relevant. Dazu kommt, dass sich die interessanten Beispiele von Unvergleichbarkeit nicht sinnvollerweise als Wahl zwischen konkreten Gegenständen oder als Wahl zwischen gewöhnlichen Konsumgütern charakterisieren lassen. In dieser Arbeit geht es nicht darum, ob man lieber Kaffee oder Tee mag. Vielmehr geht es um Entscheidungen, die für das eigene Leben eine grosse Relevanz haben können (vgl. das nächste Kapitel).

Eine Relation wird dabei nicht bloss als formale Beziehung verstanden, wie dies von einigen Philosophen, Mathematikern und Ökonomen intendiert worden ist. ${ }^{2}$ Eine Relation wird psychologisch und eine Präferenz als praktisches Urteil respektive als Evaluation aufgefasst. Wenn eine Akteurin eine Präferenz besitzt, in Bern und nicht in Basel zu wohnen, kann dies als gleichbedeutend damit verstanden werden, dass die Akteurin das Urteil fällt, Bern sei besser als dungstheorie massgeblich entwickelt haben, auf diese Weise verstanden. Savage schreibt zum Beispiel: '(...) The two assumptions just made about the relation "is not preferred to" is sometimes expressed in ordinary mathematical usage by saying that the relation is a simple ordering among acts.' (Savage 1954: 18). Falls ich es richtig sehe, übernimmt er dabei diese Deutung: '(...) Borrowing from arithmetic the suggestive abbreviation (...)' (Savage 1954: 18). 
Basel. Urteile können hinsichtlich eines bestimmten Kriteriums gebildet werden. Ein Beispiel: Die Akteurin urteilt, dass Bern hinsichtlich 'Nähe zum Arbeitsplatz' besser als Basel ist. Urteile können aber auch hinsichtlich sämtlicher relevanter Kriterien gebildet werden: Alles in allem ist es besser, in Bern als in Basel zu wohnen. Eine Präferenz meint Letzteres, wobei 'alles in allem' so verstanden werden muss, dass die Akteurin beim Vergleich der beiden Wohnorte alles oder beinahe alles berücksichtigt, was sie als relevant ansieht. Hausman schreibt: 'To say that Jill prefers $x$ to $y$ is to say that when Jill has thought about everything she takes to bar on how much she values $x$ and $y$, Jill ranks $x$ above y.' (Hausman 2012: 34). Genau auf diese Weise muss im Zusammenhang mit Unvergleichbarkeit eine Präferenz verstanden werden. Man könnte einwenden, dass Hausman respektive wir dann nicht von einer Präferenz, sondern von einem Werturteil sprechen sollten. Aber das scheint ein rein terminologischer Punkt zu sein. Ich verwende die Begriffe Evaluation, Präferenz und Werturteil im Kontext einer Entscheidung synonym. Wenn ein Akteur zwischen zwei Optionen entscheiden muss und eine Präferenz von $x$ gegenüber $y$ hat, ist dies gleichbedeutend damit, dass er das Werturteil fällt, dass $x$ besser als $y$ ist. Ich denke, dass wir uns damit nicht auf ein bestimmtes Konzept eines Werturteils festlegen. Griffin beispielsweise unterscheidet in seinem Buch Value Judgement diesbezüglich zwischen einem Wahrnehmungs- und einem Geschmacksmodell (vgl. Griffin 1996: 20). Das Wahrnehmungsmodell sagt, dass wir etwas wünschen, weil es wertvoll ist ('desired because valuable'). Das Geschmacksmodell sagt, dass wir etwas wertvoll finden, weil wir es wünschen ('valuable because desired'). Wenn die Termini Präferenz und Werturteil als gleichbedeutend verstanden werden, legt man sich nicht darauf fest, ob man etwas präferiert, weil es wertvoll ist, oder etwas wertvoll ist, weil man es präferiert. Ich will aber nicht behaupten, dass die Termini generell die gleiche Bedeutung besitzen. Ein Werturteil muss beispielsweise genau gleich wie eine Absicht nicht unbedingt eine Relation abbilden ('Freundschaft ist wichtig').

Mit dieser Deutung einer Präferenz kann der im letzten Kapitel erwähnte Disput, ob Unvergleichbarkeit zwei- oder dreistellig interpretiert werden muss, gelöst werden, oder zumindest stellt sie einen Lösungsvorschlag dar. Schaber, der Unvergleichbarkeit über 
Präferenzen charakterisiert, versteht sie als zweistelliges Konzept, Chang als dreistellig. Chang will sich nicht darauf festlegen, ob ' $x$ ist besser als $y$ ' so zu deuten ist, dass eine Akteurin $x$ gegenüber $y$ präferiert (Chang 2002b: 5). Sie würde aber sagen, dass Vergleichbarkeit auch dann ein dreistelliges Konzept darstellt, wenn 'besser als' als Präferenz verstanden wird.

Der entscheidende Punkt ist jedoch, dass eine Präferenz nicht mit einer 'einfachen' Präferenz, so wie sie Chang vermutlich versteht, gleichgesetzt wird. Bei einer Präferenz in unserem Sinne sind die entscheidenden und von Chang geforderten Relativierungen enthalten. (Un)Vergleichbarkeit beinhaltet dreistellige, 'einfache' Präferenzen und endet eben nicht in einer zweistelligen, allgemeinen Präferenz. Hierbei ist es durchaus möglich, dass eine Akteurin Bern gegenüber Basel bevorzugt, weil ihr Freund in Bern wohnt, und Basel gegenüber Bern präferiert, weil es in Basel fantastische Museen gibt. Solche Präferenzen sind nicht entscheidend, ob Unvergleichbarkeit vorliegt oder nicht. Ausschlaggebend ist, ob sich eine Präferenz formulieren lässt, in welcher Gründe wie 'der Freund wohnt in Bern' und 'Basel hat fantastische Museen' gegeneinander abgewogen werden können.

Die vorgeschlagene Präferenzversion von Unvergleichbarkeit sagt dementsprechend nicht, dass Unvergleichbarkeit mit unmittelbaren und unreflektierten Einstellungen zu tun hat. Wenn eine Akteurin nicht sagen kann, ob sie lieber Ökonomie oder Rechtswissenschaft studieren will, weil sie sich zur entsprechenden Entscheidungssituation noch überhaupt nichts überlegt hat, hat dies nichts mit Unvergleichbarkeit zu tun. Und wenn eine Akteurin weder eine Pro-Einstellung zugunsten der einen Option besitzt noch gleichgültig hinsichtlich der Optionen ist, aber nach einem vertieften Nachdenken - das heisst unter anderem: nach einer Abwägung von Gründen zum Schluss kommt, dass sie lieber Ökonomie als Rechtswissenschaft studieren möchte, dann liegt ebenfalls keine Unvergleichbarkeit vor.

Unvergleichbarkeit hat dementsprechend weder mit spontanen Reaktionen noch damit zu tun, dass die Optionen noch in keine Reihenfolge gebracht wurden, sondern damit, dass die Optionen nicht in eine Reihenfolge gebracht werden können.

Wie aber hängen Präferenzen und Gründe zusammen, und welche Sichtweise des praktischen Überlegens spielt dabei eine Rolle? 
Bevor im nächsten Teil dieser Frage nachgegangen wird, muss auf einen ernstzunehmenden Einwand gegen die vorgeschlagene Deutung einer Präferenz eingegangen werden.

Eine Präferenz, so wie der Begriff in diesem Teil erläutert worden ist, stellt das Resultat eines anspruchsvollen kognitiven Prozesses dar. Der Akteur muss bei der Evaluation alles berücksichtigen, was er für die Entscheidungssituation als relevant ansieht. Anders als die Begriffe der Unvergleichbarkeit und der Inkommensurabilität stellt der Terminus der Präferenz aber eher einen umgangssprachlichen Begriff dar, auch wenn der Kellner in England wohl fragt 'Which would you like?' und nicht 'Which would you prefer?' (Hausman 2012: 1). Dies stellt deshalb ein Problem dar, weil der Alltagsgebrauch nicht sagt, dass eine Präferenz das Resultat einer intensiven Abwägung ist - in dem Sinne, dass relevante Gründe gegeneinander abgewogen werden. Es lässt sich nicht bestreiten, dass von einem umgangssprachlichen Verständnis abgewichen wird. Um das Phänomen der Unvergleichbarkeit erfassen zu können, ist es allerdings angebracht. Die Abweichung von möglichen umgangssprachlichen Verwendungsweisen ist theoretisch gerechtfertigt.

\subsection{Präferenzen, Gründe und praktisches Nachdenken}

Im Alltag können wir oft auf der Basis bekannter Vorlieben entscheiden. Manchmal sind bestehende Präferenzen aber nicht hinreichend, um ein Entscheidungsproblem lösen zu können. In der Psychologie spricht man von preference construction: '(...) in many situations we do not really know what we prefer, we must construct our preferences as the situation arises.' (Lichtenstein und Slovic 2006: 1).

Der Umstand, dass Präferenzen manchmal konstruiert werden müssen, scheint innerhalb der Psychologie einen Konsens darzustellen. Die aktuelle Debatte dreht sich vielmehr um die Frage, unter welchen Bedingungen dies der Fall ist (vgl. etwa Warren, McGraw und Van Boven 2011).

Die Konstruktion kann in verschiedenen Situationen vorkommen, zum Beispiel, dass man zwischen verschiedenen Menüs wählen muss, ohne die chinesische Speisekarte zu verstehen. Oder auch in 
Fällen, in denen wir nicht wissen, wie wir unterschiedliche Überlegungen gegeneinander abwägen sollen. Das Phänomen der Unvergleichbarkeit ist eher in letzterem Bereich anzusiedeln. Es geht nicht um Fälle völliger Ratlosigkeit, in denen überhaupt keine handlungsleitenden Gründe herangezogen werden können (die chinesische Speisekarte), sondern um Kasus, in denen die Fakten einigermassen bekannt sind, die für die unterschiedlichen Optionen sprechen.

Es ist jedoch wichtig zu erwähnen, dass wir (i) eine Präferenz nicht so verstehen, wie das die Psychologen oft tun ${ }^{3}$, und (ii) der entscheidende Punkt darin besteht, dass die Deliberation im Falle von Unvergleichbarkeit in keinem Urteil endet und überhaupt keine Präferenz konstruiert werden kann.

Sämtliche zur Wahl stehenden Alternativen erscheinen fragwürdig. Man befindet sich in einem Zustand der Unentschiedenheit, nicht in dem Sinne, dass man sich dazu entschlossen hat, es sei egal, ob man Rechtswissenschaft oder Ökonomie studieren will, sondern in dem Sinne, dass man sich dazu entschieden hat, unentschieden zu sein. Die Unentschiedenheit besteht darin, dass man nicht angeben kann, für welche Wahl man die besseren Gründe besitzt.

Die Charakterisierung von Unvergleichbarkeit über Gründe ist deshalb sinnvoll, weil ein Entscheidungskonflikt oftmals dann entsteht, wenn man gute Gründe für oder gegen die zur Auswahl stehenden Optionen besitzt und nicht sagen kann, wie diese gegeneinander abgewogen werden sollen. ${ }^{4}$

Der Leser mag einwenden, dass die Rede von Gründen charakteristisch für die Philosophie ist und wenig mit unserem Alltag zu tun hat. Es ist ziemlich klar, dass Philosophinnen und Philosophen die Tendenz haben, überrationalisiert zu sein. Der Punkt aber, dass der consider something desirable or undesirable.' (Warren, McGraw und Van Boven 2011: 194). Unter einer Präferenz wird wie gesagt eine Evaluation verstanden, welche Option alles in allem besser ist.

4 Auch in der Psychologie lässt sich Evidenz finden, dass es sinnvoll sein kann, sogenannte pre-decisional conflicts - damit sind Entscheidungskonflikte gemeint, in denen wir uns noch nicht für oder gegen eine Option entschieden haben - anhand von Gründen zu charakterisieren (Shafir, Simonson und Tversky 2006). Shafir, Simonson und Tversky gehen davon aus, dass ein Konflikt entsteht, weil man wählen muss. Dabei argumentieren sie, dass wir genau dann nach Gründen suchen, wenn wir keiner Option mehr Wert zuschreiben. 
Gegenstand der Abwägung nicht die Präferenzen selbst sind, scheint plausibel zu sein. Präferenzen und deren Ursachen sind im Normalfall nicht Thema der Deliberation. Der Gegenstand der Deliberation ist vielmehr, was präferiert wird. Die Unvergleichbarkeit - der Umstand, dass keine Präferenz formuliert werden kann - steht am Schluss und nicht am Anfang des Deliberationsprozesses. Man kann sagen, dass eine Präferenz in diesem Zusammenhang die Vergleichbarkeit der Werte voraussetzt. Wenn eine Akteurin die Wohnorte Bern und Basel nicht vergleichen kann, heisst dies, dass sie nicht sagen kann, wo sie wohnen will und ob Bern oder Basel an erster Stelle steht. Der Gegenstand der Deliberation aber sind nicht die eigenen Präferenzen, es sind die Gründe oder Werte, welche für Bern oder Basel sprechen: Bern liegt an der Aare; der Freund wohnt in Bern; in Basel gibt es fantastische Museen und die leckersten Kebabs. Die Frage, wie Werte und Gründe zusammenhängen, spielt meines Erachtens für uns keine Rolle. Vielmehr wird uns im nächsten Kapitel beschäftigen, was es genau heisst, dass Gründe oder Werte nicht gegeneinander abgewogen werden können. Ist damit beispielsweise gemeint, dass man diese verschiedenen Dinge wertschätzt? Oder wird damit ausgedrückt, dass man nicht weiss, wie hoch man die verschiedenen Dinge wertschätzt?

In diesem Kapitel interessiert jedoch nicht eine Präzisierung des Phänomens der Unvergleichbarkeit. Von Bedeutung ist die allgemeine Frage, welcher Ansatz des praktischen Nachdenkens im Zusammenhang mit Präferenzen relevant ist.

Bevor dieser Frage nachgegangen wird, muss kurz auf die Unterscheidung zwischen einer 'einfachen' Präferenz und einem Grund eingegangen werden. Weshalb wird der Deliberationsprozess nicht nur anhand von Präferenzen beschrieben? Könnte man nicht sagen, dass im Falle von Unvergleichbarkeit die Akteurin Bern gegenüber Basel präferiert, weil ihr Freund in Bern wohnt, und Basel gegenüber Bern präferiert, weil es in Basel fantastische Museen gibt? Anders formuliert: Könnte man nicht sagen, dass 'einfache' Präferenzen für unterschiedliche Optionen sprechen und deshalb keine allgemeine Präferenz erfolgen kann? Die Idee, weshalb die Charakterisierung über Gründe mehr Sinn ergibt, ist die folgende: Eine Akteurin kann eine 'einfache' Präferenz (im Sinne eines Wunsches oder einer Pro-Einstellung) besitzen, ein Museum zu besuchen anstatt ins Kino zu gehen. 
Die Tatsache, dass es in Basel fantastische Museen gibt, kann aber auch einen Grund darstellen, in Basel und nicht in Bern zu wohnen. Die Unterscheidung zwischen einem Grund und einer 'einfachen' Präferenz hat also weniger mit dem Inhalt (das Museum, der Freund) als vielmehr damit zu tun, wie darüber nachgedacht wird. Es geht um die Perspektive. Eine 'einfache' Präferenz kann so verstanden werden, dass nicht bestimmt werden kann, ob sie handlungswirksam ist oder nicht. Man besitzt nun einmal den Wunsch, ins Museum zu gehen. Gründe hingegen können so interpretiert werden, dass bestimmt werden kann, ob sie handlungsrelevant sind oder nicht (vgl. Davidson 1963). Man weiss, dass es in Basel gute Museen gibt, und kann bestimmen, ob dies handlungsrelevant ist oder nicht. So kann man sich beispielsweise auch das Gegenteil oder kontrafaktische Szenarien vorstellen: Der Umstand, dass es in Basel fantastische Museen gibt, spricht dagegen, in Basel zu wohnen. Oder: Möchte ich immer noch in Basel wohnen, wenn es dort keine fantastischen Museen gäbe? Dies führt uns zur Frage, welche Theorie des praktischen Überlegens im Zusammenhang mit dem Phänomen der Unvergleichbarkeit relevant ist. Praktisches Überlegen kann dabei als Fähigkeit verstanden werden, dass man durch Nachdenken die Frage, was man tun will oder soll, lösen kann. Das Nachdenken ist praktisch, insofern es sich um Nachdenken über Handlungen handelt, aber auch insofern die Konsequenz des Nachdenkens eine Handlung ist.

Auf jeden Fall legt man sich mit einer Präferenzversion von Unvergleichbarkeit nicht auf ein fragwürdiges Verständnis präferenzbasierter Entscheidungstheorien fest. Man fixiert sich nicht darauf, dass axiomatische Entscheidungstheorien, die mit dem Begriff der Präferenz operieren, auch etwas dazu sagen können, was wirklich wichtig ist und welche Präferenz ein Akteur bilden soll. Der Sinn moderner Entscheidungstheorien besteht u. a. darin, Anforderungen zu formulieren, in welchem Verhältnis Präferenzen stehen oder stehen sollen (Transitivität, Reflexivität etc.). Hinsichtlich dieses Teils der Deliberation - welche Dinge will man wirklich tun?, und das ist an dieser Stelle der entscheidende Punkt - schweigen solche Theorien aber. Deshalb stellen diese Modelle auch keine vollständigen Theorien des praktischen Überlegens dar. Etwas anderes zu behaupten, wäre nach Richardson ungefähr so, wie wenn ein Atlas als vollständige Theorie 
des Reisens angesehen würde, auch wenn dieser nichts darüber sagt, wohin man gehen soll (Richardson 1994: 9).

Kauft man sich mit einer Präferenzversion aber nicht die Prämisse ein, dass praktisches Nachdenken instrumentell ist? Was bedeutet in diesem Kontext instrumentell? Es geht darum, ob die Präferenz selbst Gegenstand rationaler Überlegungen sein kann oder nicht. Der Instrumentalismus besagt, dass Präferenzen nicht Gegenstand der Vernunft sind und sich praktisches Nachdenken auf die Umsetzung von Präferenzen beschränkt. Man kann dies so verstehen, dass der Inhalt der Präferenz als gegeben angesehen wird. Hume brachte dies mit folgendem Zitat auf den Punkt: 'It is not contrary to reason to prefer the destruction of the whole world to the scratching of my finger.'(Hume 1986: 416). Die Fähigkeit, vernünftig zu überlegen, kommt nach Hume erst dann ins Spiel, wenn es darum geht, wie die Präferenz (die Vernichtung der Menschheit) erfüllt werden kann. Wenn wir nochmals obiges Bild des Atlas verwenden, heisst dies, dass das Reiseziel jeweils schon bekannt ist. Die praktische Vernunft sagt einem dann 'nur' noch, wie man auf die Bahamas kommt. Sie stellt ein Instrument dar, wie die Präferenz erfüllt werden kann. Dieser Position zufolge geht es beim praktischen Überlegen darum herauszufinden, wie man bekommt, was man will. Es kann sich aber nicht darum drehen, was man wünschen sollte. In einem gewissen Sinne finden wir diese Annahme auch in der Entscheidungstheorie, zum Beispiel in der mikroökonomischen Präferenztheorie. Damit meine ich, dass die Präferenzen und somit auch die Nutzenfunktion eines Akteurs in der Entscheidungstheorie als gegeben angesehen werden. Die Frage, welche Präferenz angebracht ist, ist, wie weiter oben erwähnt, nicht Gegenstand eines solchen Modells. ${ }^{5}$

Den Instrumentalismus im praktischen Nachdenken finden wir aber nicht nur bei Hume oder in der Entscheidungstheorie. Er liegt auch der sogenannten Zweckrationalität oder neueren Modellen der Rationalität zugrunde. Ich meine, dass der Instrumentalismus im

5 Die Parallele zwischen der modernen Entscheidungstheorie und Hume besteht darin, dass eine einzelne Präferenz nicht irrational sein kann bzw. einzelne Präferenzen alle gleich rational sind. Der Unterschied zu Hume besteht darin, dass in der Entscheidungstheorie mehrere Präferenzen zusammen sehr wohl irrational sein können, zum Beispiel wenn sie nicht transitiv sind. 
ersten Fall besagt, dass Zwecke als gegeben angesehen werden. Im zweiten Fall sind es dann beispielsweise Absichten, die nicht Gegenstand rationaler Überlegungen sind. Aristoteles schrieb zum Beispiel: 'Gegenstand der Überlegung sind aber nicht die Ziele, sondern das, was zu den Zielen führt.' (Aristoteles 2008: 1112b). In englischsprachigen Versionen der Nikomachischen Ethik finden wir folgende prägnante Übersetzung: 'We deliberate not about ends but about what contributes to ends.' (Aristotle 1984: 1112b). Man kann auch sagen, dass sich die Normativität bei der Zweckrationalität nicht auf den Zweck selbst, sondern auf die Handlung bezieht, wie der Zweck erfüllt werden kann. Ein Beispiel: Das erstrebte Ziel von Simon besteht darin, so schnell wie möglich ein Schokoladeneis zu essen. Simon glaubt, dass das beste Mittel zum Zweck darin besteht, eine Gelateria aufzusuchen. Ergo soll Simon diese bestmögliche Handlung ausführen. Inwiefern man dies tatsächlich tun soll, ist in einer zeitgenössischen Diskussion in Kritik geraten. Die streitbaren Philosophen fragen dann: Warum sollen wir beabsichtigen, eine Gelateria aufzusuchen, wenn wir beabsichtigen, ein Eis zu essen, und überzeugt sind, dass der Besuch der Gelateria notwendig ist, um ein Eis essen zu können? Der entscheidende Punkt ist aber, dass auch hier der Inhalt der Absicht (Schokoladeneis zu essen) nicht Gegenstand des Modells ist.

Weshalb jedoch sollte über den Inhalt einer Absicht oder eines Zwecks oder eben einer Präferenz nicht nachgedacht werden können? Der Instrumentalismus kann nicht den ganzen Gegenstandsbereich des praktischen Überlegens erfassen. Die vorgeschlagene Präferenzversion von Unvergleichbarkeit legt den Schluss nahe, dass praktisches Nachdenken nicht nur instrumentell ist. Bei der Konstruktion einer Präferenz geht es nämlich um deren Inhalt. Es geht darum, eine Präferenz zu bilden, sie wird nicht als gegeben angesehen. Ich werde diesen Punkt im Kapitel Unvergleichbarkeit und Rationalität vertiefen. An dieser Stelle soll nur das wichtigste Argument genannt werden, weshalb eine präferenzbasierte Sichtweise von Unvergleichbarkeit nicht auf ein instrumentelles Nachdenken reduziert werden kann: Es lassen sich Gründe dafür und dagegen nennen, ob man auf die Bahamas fliegt. Man kann dies alleine in Gedanken durchspielen oder in Gesprächen mit Mitmenschen diskutieren. Diese Fähigkeit des praktischen Überlegens, welche im Zusammenhang mit Unvergleichbarkeit 
relevant ist, besitzen wir zweifellos. Wie oft wir diese Fähigkeit auch anwenden, ist notabene eine andere Frage.

Zusammenfassend kann festgehalten werden, dass eine präferenzbasierte Sichtweise von Unvergleichbarkeit nicht-instrumentelles Nachdenken darüber beinhaltet, welche Präferenz angebracht ist, wobei die Präferenz in einem gewissen Sinne den Endpunkt der Überlegung darstellt. Im Falle von Unvergleichbarkeit gelangt man hierbei zu keinem Ergebnis beziehungsweise es kann keine Präferenz konstruiert werden.

\subsection{Mögliche Einwände und Abgrenzung}

Auch wenn im Deliberationsprozess Gründe gegeneinander abgewogen werden, geht die vorgeschlagene Präferenzversion davon aus, dass Unvergleichbarkeit letztlich als subjektive Bewertung eines beliebigen Akteurs angesehen werden kann. In dieser Hinsicht wird wie in Schauer 1998 angenommen, dass (i) Unvergleichbarkeit nicht zwingend etwas mit moralischer Ontologie, sondern vielmehr mit Einstellungen, Dispositionen etc. zu tun hat und (ii) nicht unabhängig von einer einzelnen Akteurin vorkommt. Das subjektive Element wird dabei am Terminus der Präferenz festgemacht. Die blosse Bezugnahme auf Präferenzen impliziert notabene nicht unbedingt eine subjektivistische Lesart. Eine Präferenz kann auch als Einstellung verstanden werden, die man haben soll. Es kann spezifischer an eine Präferenz gedacht werden, die ein nicht-willensschwacher Akteur haben, oder an eine Präferenz, die ein vollständig informierter Akteur angeben würde.

Schaber definiert Unvergleichbarkeit respektive Inkommensurabilität - er versteht die Begriffe synonym - auf diese Weise: 'The incommensurability of certain options is based on the fact that preferences are sometimes not rankable, even if the agent is fully informed about the options in question.' (Schaber 1994: 153). Dies stellt meines Erachtens jedoch eine unzureichende Charakterisierung von Unvergleichbarkeit dar.

Einerseits sollte die zusätzliche Bedingung der vollständigen Information nicht verwendet werden, damit die Alltagsphänomenologie besser erfasst werden kann. Die Even-if-Bedingung in Schabers 
Definition ist beinahe nie erfüllt. In der Konsequenz heisst dies, dass es gemäss Schabers Definition kein reales Phänomen der Unvergleichbarkeit gibt. Wir sind in den meisten Fällen nämlich nicht vollständig über die Konsequenzen unserer Handlungen informiert. ${ }^{6}$

Andererseits, treffen die Überlegungen im letzten Abschnitt zu, besteht Unvergleichbarkeit streng genommen nicht darin, dass bestehende Präferenzen in keine Rangordnung gebracht werden können, sondern darin, dass überhaupt keine Präferenz konstruiert werden kann.

Ein potenzieller Einwand kann nun darin bestehen, dass dies ein zu wenig objektives Konzept von Unvergleichbarkeit darstellt. Deshalb, weil die Sätze 'Option $x$ ist besser als Option $y$ ' oder 'Option $x$ ist weder besser noch schlechter als noch gleich gut wie Option $y$ ' unabhängig von einer bestimmten Akteurin wahr oder falsch sein können. Dies würde bedeuten, dass Optionen in einem bestimmten Zusammenhang stehen und das Bestehen dieses Zusammenhangs unabhängig von den Präferenzen eines spezifischen Akteurs gegeben ist. So könnte zum Beispiel der Satz 'Ein Sonnenaufgang ist besser als ein Sonnenuntergang' unabhängig von einem bestimmten Akteur wahr oder falsch sein. Oder der Satz 'Leben ist besser als Tod' könnte beispielsweise unabhängig von einem bestimmten Akteur wahr oder falsch sein. Chang und Raz können zum Teil auf diese Weise gelesen werden. ${ }^{7}$ Chang scheint in ihrer Argumentation teilweise implizit davon auszugehen, dass es eine Tatsache in der Welt gibt, dass zum Beispiel $x$ nicht besser als $y$ ist. Das Konzept der (Un)Vergleichbarkeit müsste dementsprechend objektiv und nicht subjektiv verstanden werden. 'Objektiv' heisst in diesem Zusammenhang 'unabhängig vom Akteur gegeben/nicht gegeben'. 'Subjektiv' heisst 'abhängig vom Akteur der Fall oder nicht der Fall'. Philosophinnen und Philosophen, die die Optionen informiert ist, aber nicht behauptet, dass er auch über alle Konsequenzen Bescheid weiss. Auf jeden Fall spielen sogenannte faktische Unsicherheiten - man weiss nicht genau, was nach der getroffenen Entscheidung passiert - eine Rolle, weshalb Optionen zum Zeitpunkt einer Entscheidung manchmal nicht eindeutig als besser/schlechter/gleich gut bewertet werden können. Das heisst aber nicht, und darauf weist Schabers Klausel zu Recht hin, dass Unvergleichbarkeit durch faktische Unsicherheit erklärbar ist. Darauf wird im nächsten Kapitel eingegangen. 
für eine objektive Position einstehen, sind oftmals der Meinung, dass Dinge einen Wert haben können, auch wenn ihnen niemand einen Wert zuschreibt. Der Satz 'Option $x$ ist besser als Option $y$ ' bedeutet demnach, dass Option $x$ mehr Wert als Option $y$ besitzt, wobei ein Wert als etwas Subjektunabhängiges aufgefasst werden muss. Eine Konzeption von Unvergleichbarkeit müsste demnach so formuliert werden, dass eine Option weder mehr noch weniger noch gleich viel Wert wie eine andere Option besitzt, wobei natürlich expliziert werden müsste, was mit einem objektiven Wert genau gemeint ist.

Diesem potenziellen Einwand kann auf verschiedene Arten begegnet werden:

Eine Möglichkeit besteht darin, Argumente zu nennen, warum es problematisch ist, dass ' $x$ ist besser als $y$ ' unabhängig von einem bestimmten Akteur wahr oder falsch sein kann. Zum Beispiel, weil damit eine Wert-Ontologie impliziert wird, in welcher Werte ausserhalb des Gehirns einer spezifischen Akteurin lokalisiert werden müssen. Oder man könnte argumentieren, dass eine objektive Wertauffassung zwar nicht unbedingt einen Wertrealismus impliziert, dass aber dennoch daraus folgt, dass Dinge einen Wert haben können, auch wenn kein Bewusstsein existiert und es nicht plausibel ist anzunehmen, dass Sonnenaufgänge mehr Wert als Sonnenuntergänge haben, wenn keine Person existiert, die ersteren mehr Wert zuschreibt.

Eine andere Möglichkeit besteht darin, für eine Position einzutreten, in welcher Werte über Wertschätzungen und Wertschätzungen über Präferenzen definiert werden. Gemäss dieser Sichtweise gilt Folgendes: $x$ hat einen Wert genau dann, wenn ein Akteur $\mathrm{A} x$ als wertvoll ansieht. Ein Akteur A sieht $x$ als wertvoll an genau dann, wenn A $x$ gegenüber nicht $x$ präferiert. Ein Beispiel: Vanilleeis zu essen hat einen Wert, weil A 'Vanilleeis Essen' als etwas Wertvolles ansieht, und dies bedeutet, dass A 'Vanilleeis Essen' gegenüber 'Nicht Vanilleeis Essen' präferiert. A zieht eine Welt mit Vanilleeis gegenüber einer Welt ohne Vanilleeis vor. Ein Sachverhalt $x$ ist gemäss dieser Auffassung genau dann wertvoll, wenn der Existenz von $x$ gegenüber der Nichtexistenz von $x$ Vorzug gebührt, selbst für den Fall, dass ausser $x$ kein Sachverhalt existiert. Wenn A $x$ gegenüber $y$ bevorzugt, bedeutet dies, dass A die Existenz von $x$ der Existenz von $y$ vorzieht. Werte drücken nach dieser Auffassung die Interessen eines bestimmten Akteurs aus. Dass 
$x$ Wert hat, ist gemäss dieser Sichtweise gleichbedeutend damit, dass $x$ von einem Akteur als wertvoll beurteilt wird (Seel 1977: 128, 129).

Eine dritte Möglichkeit - und dafür wird an dieser Stelle plädiert - ist, darauf hinzuweisen, dass mit einer Präferenzversion von Unvergleichbarkeit im ungünstigsten Fall ein anderes Phänomen untersucht wird. Bei der Erscheinung der Unvergleichbarkeit geht es darum, wie man zu Präferenzen gelangt beziehungsweise wie eben nicht. Gründe oder Werte spielen dabei wohlgemerkt insofern die zentrale Rolle, als diese manchmal nicht gegeneinander abgewogen werden können. Dies führt bisweilen dazu, dass ein Akteur keine Präferenz konstruieren kann. Aber es ist richtig, dass Unvergleichbarkeit gemäss der in diesem Kapitel entwickelten Sichtweise am Schluss damit steht und fällt, ob eine Präferenz gebildet werden kann oder nicht. Durch die Bezugnahme auf Präferenzen kommt dem Phänomen der Unvergleichbarkeit letztlich ein irreduzibel subjektives Element zu. Man kann mit Tugendhat auch sagen, dass am letzten Punkt des praktischen Nachdenkens ein irreduzibel subjektives Element steht: der Akteur.

Ein weiterer potenzieller Einwand lautet, dass eine Akteurin eine Präferenz von $x$ gegenüber $y$ besitzen kann, aber dennoch Unvergleichbarkeit vorliegt. Ein Beispiel: Ein Akteur studiert alles in allem lieber Ökonomie als Jurisprudenz, aber dennoch sind die beiden Studiengänge unvergleichbar. Wie liesse sich dieser Einspruch plausibilisieren? Man könnte einen Ansatz von Unvergleichbarkeit entwickeln, in dem Unvergleichbarkeit nichts mit einem spezifischen Akteur zu tun hat. Man könnte eine Theorie vertreten, in der das Studium der Rechtswissenschaft und dasjenige der Ökonomie für sämtliche Akteure unvergleichbar sind. Zum Beispiel, weil es bei der Studienwahl per se unmöglich ist, einen übergeordneten Wert zur Entscheidungsfindung beizuziehen. Oder man könnte argumentieren, dass unvergleichbare metaphysische Werte existieren, welche in beiden Studiengängen unterschiedlich realisiert werden, und deshalb die Optionen unvergleichbar sind. Dinge könnten dann unvergleichbar sein, auch wenn es der Akteur anders sieht oder nicht erkennt.

Die Antwort auf diesen Einwand fällt ähnlich wie die vorherige Erwiderung aus: Man operiert in diesem Fall mit einem anderen Begriff. Falls die Überlegungen im ersten Kapitel zutreffen - Unvergleichbarkeit ist ein technischer Begriff -, stellt es keinen paradigmatischen 
Fall von Unvergleichbarkeit dar, dass ein Studium der Ökonomie und ein Studium der Rechtswissenschaft unvergleichbar sind, und gemäss einer Präferenzversion fälschlicherweise Vergleichbarkeit folgt. Obiger metaphysischer Ansatz würde ein anderes Konzept von Unvergleichbarkeit darstellen. Ich will nicht behaupten, dass Unvergleichbarkeit nicht auch als metaphysisches Problem angesehen werden kann, in dem Sinne, dass es unabhängig von einem bestimmten Akteur besteht. Gegen eine solche Herangehensweise sprechen jedoch zwei Argumente: Einerseits sind es aus der Perspektive des Akteurs keine metaphysischen Überlegungen, weshalb Unvergleichbarkeit ein Problem darstellt. ${ }^{8}$ Andererseits stellt es eine unplausible Konsequenz dar, dass das Studium der Ökonomie und dasjenige der Rechtswissenschaft für sämtliche Akteure (un)vergleichbar sein sollen. Für die einen ist es eindeutig, was sie studieren wollen, und für andere nicht (ich komme gleich nochmals darauf zurück).

Es muss allerdings eingeräumt werden, dass der vorgeschlagene Ansatz nicht erfassen kann, dass oft gar nicht gut für uns ist, was wir wollen. Es ist denkbar, dass das Studium der Rechtswissenschaft und dasjenige der Ökonomie für eine Akteurin unvergleichbar sind, es aber besser wäre, wenn sie die Jurisprudenz bevorzugen würde, zum Beispiel weil sie hierfür mehr Talent aufbringt. Ich denke, dass objektive Kriterien des guten Lebens existieren, in dem Sinne beispielsweise, dass psychologische Studien zeigen, dass es wichtig für die eigene Zufriedenheit ist, bei einer Tätigkeit weder über- noch unterfordert zu sein, und es erstaunlich wäre, wenn man selbst eine Ausnahme darstellen würde, also zugleich zufrieden und massiv überfordert sein könnte. Es ist möglich, dass eine Person nach reiflicher Überlegung zum Schluss kommt, dass sie nicht angeben kann, ob sie lieber Ökonomie oder Jurisprudenz studieren will, dies aber nicht dem wahren Interesse der Person entspricht, weil sie dann zum Beispiel überfordert wäre. Diesen Einwand muss ich akzeptieren. Würde man Unvergleichbarkeit anhand des Begriffs des wahren Interesses charakterisieren, ergäben sich andere Schwierigkeiten (Wer bestimmt das wahre Interesse? Worin besteht der Zusammenhang zur realen Person und ihren aktuellen Urteilen, Interessen und Werten?). 
In diesem Zusammenhang zeigen sich zwei wichtige Punkte:

Erstens: Unvergleichbarkeit hat immer mit einem unwissenden Akteur zu tun, der unter Umständen Präferenzen konstruiert, die ihm gar nicht gut tun. Diese Konsequenz nehmen wir mit unserem Ansatz in Kauf. Ich kann nicht ausschliessen, dass die Präferenzen des Akteurs und dessen Wohlergehen auseinanderlaufen. Die Präferenzen müssen dabei nicht stabil sein. Eine Akteurin kann die Optionen, ob sie in Bern oder Basel wohnen will, zu einem Zeitpunkt als unvergleichbar und zu einem späteren Zeitpunkt durch ein Ändern ihrer Wünsche, Ziele, Wertvorstellungen etc. als vergleichbar ansehen. Die entwickelte Präferenzversion impliziert nicht zwingend, dass sich die Unvergleichbarkeit zu einem späteren Zeitpunkt nicht auflösen lässt. Sie sagt, dass die Optionen zum Zeitpunkt der Entscheidung unvergleichbar sind.

Zweitens: Eine Präferenzversion von Unvergleichbarkeit, die im nächsten Kapitel weiter geschärft wird, hat, wie im letzten Kapitel dargelegt wurde, zum Ziel, ein bestimmtes reales Phänomen zu erfassen. Dabei geht es nicht um die Beschreibung eines normativen Ideals. Diese Arbeit sagt nicht: Du sollst bestimmte Dinge, etwa die Studienwahl, als unvergleichbar betrachten. Wir alle beurteilen Optionen sowieso manchmal als unvergleichbar, wenn auch nicht zwingend in den gleichen Entscheidungssituationen. Aus diesem Grund wird keine Version vertreten, welche besagt, dass man im Falle von Unvergleichbarkeit weder eine spezifische Präferenz besitzen noch gleichgültig sein soll, oder dass man dazu verpflichtet ist, weder eine Präferenz zu haben noch indifferent zu sein. Das Phänomen der Unvergleichbarkeit impliziert aber auf jeden Fall eine gewisse Form der Normativität, in dem Sinne, dass am Anfang die Frage steht, was man tun will beziehungsweise was das Beste zu tun ist.

\subsection{Zusammenfassung}

Im ersten Teil wurde eine Präferenzversion von Unvergleichbarkeit eingeführt. Zwei Optionen sind demnach genau dann unvergleichbar, wenn eine Akteurin keine Präferenz zugunsten der einen Option besitzt und auch nicht gleichgültig zwischen den zur Wahl 
stehenden Alternativen ist. Ich habe argumentiert, dass eine Präferenz auf ganz bestimmte Weise verstanden werden sollte, nämlich so, dass für den Akteur eine Option alles in allem besser ist.

Im Falle von Unvergleichbarkeit, dafür wurde im zweiten Teil argumentiert, endet die Deliberation in keiner Präferenz. Man kann nicht sagen, welche Option besser ist, und findet sich in einem $\mathrm{Zu}-$ stand der Unentschiedenheit wieder. Dies kann so interpretiert werden, dass man nicht angeben kann, für welche Wahl man die besseren Gründe besitzt. In diesem Zusammenhang wurde erläutert, weshalb dies eine nicht-instrumentelle Auffassung des praktischen Nachdenkens bedingt. Die Begründung dafür ist, dass es bei der Konstruktion einer Präferenz um deren Inhalt geht. Instrumentelle Modelle hingegen setzen den Inhalt der Einstellung als gegeben voraus.

Im dritten Teil wurden potenzielle Argumente gegen eine Präferenzversion diskutiert, wie zum Beispiel, dass Unvergleichbarkeit unabhängig von Präferenzen besteht.

Worin liegen die Vorteile einer Präferenzversion von Unvergleichbarkeit? Das stärkste Argument für eine solche Betrachtungsweise besteht darin, dass sie für unseren Alltag relevant ist. Bei anderen Ansätzen - zum Beispiel: zwei abstrakte Werte sind inkompatibel; Gegenstände lassen sich nicht hinsichtlich einer numerischen Skala vergleichen - ist die alltägliche Relevanz weniger klar. Eine Präferenzversion trägt dem Anspruch der sogenannten phänomenalen Korrektheit Rechnung. Was ist gemeint? Um dem Phänomen der Unvergleichbarkeit gerecht zu werden, so die Idee, muss die einzelne Person 'ernst genommen werden'. Dies kann so verstanden werden, dass es wichtig ist, wie der einzelne Akteur die Welt wahrnimmt. Eine Präferenzversion erfüllt dieses Kriterium. Sie stellt keine Irrtumstheorie von Unvergleichbarkeit dar. Dies ist deshalb ein Vorteil, weil eine Irrtumstheorie ceteris paribus schlecht ist. Wenn Philosophen Unvergleichbarkeit zum Beispiel auf sprachphilosophische Vagheit reduzieren wollen - in diesem Bereich werden neue und hoch spezialisierte Aufsätze publiziert, deren Relevanz meines Erachtens sowohl für Laien als auch für Philosophen nicht mehr nachvollziehbar ist -, sollte dies ernst genommen, aber auch skeptisch betrachtet werden. ${ }^{9}$ 
Man kann sagen, dass phänomenale Nicht-Korrektheit prima facie gegen eine philosophische Theorie spricht. Der einzelne Akteur nimmt Unvergleichbarkeit nicht als sprachphilosophisches Problem wahr. Das heisst nicht, dass Sprache keinen Einfluss auf Entscheidungen haben kann. Dies zu behaupten, wäre falsch. ${ }^{10}$ Es ist denkbar, dass die Art der Beschreibung einer Situation Einfluss darauf hat, ob ein Akteur zwei Optionen als vergleichbar oder unvergleichbar ansieht. Man müsste dies experimentell untersuchen, was bisher nicht gemacht wurde. Auch entwickle ich in diesem Buch in Kapitel 7 eine Theorie von vagen Entscheidungskriterien, die für das Phänomen der Unvergleichbarkeit relevant ist. Mein Punkt an dieser Stelle ist jedoch, dass Unvergleichbarkeit aus der Perspektive des Akteurs, zum Beispiel bei der Wahl des Studiums, nicht deshalb eine Schwierigkeit darstellt, weil umgangssprachliche Begriffe Grenzfälle zulassen. Der unbestreitbare Fakt, dass unsere Umgangssprache vage ist, scheint höchstens einen marginalen Einfluss darauf zu haben, dass ein Akteur nicht weiss, ob er Ökonomie oder Rechtswissenschaft studieren will. Wenn das Gegenteil der Fall wäre, würde Unvergleichbarkeit verschwinden, wenn wir eine präzisere Sprache besässen. Das scheint unplausibel zu sein. Selbst dann könnte ein Akteur manchmal keine Präferenz bilden.

Das bedeutet wohlgemerkt nicht, dass die bisherige phänomenale Beschreibung von Unvergleichbarkeit genügend präzise ist. Und es heisst nicht, dass hinreichend erläutert worden ist, weshalb ein Akteur keine Präferenz konstruieren kann. Wenn es sich so zu

Deutung zufolge ist das Phänomen der Inkommensurabilität bzw. Unvergleichbarkeit auf die Vagheit mancher unserer alltagssprachlichen, evaluativen Begriffe zurückzuführen. Dass viele evaluative Begriffe vage sind, bedeutet, dass sie in Grenzfällen nicht eindeutig wahr oder falsch sind. Im Falle von Unvergleichbarkeit haben wir es dieser Irrtumstheorie zufolge mit Grenzfällen eines evaluativen Komparativs wie 'ist gleich gut' zu tun (die Vergleichsrelation 'gleich gut' besteht nur scheinbar nicht). Broome nennt diese Art der Vagheit soft-indeterminacy. Mithilfe einer bekannten Vagheitstheorie, dem Supervaluationismus - dieser Name geht unter anderem auf Van Fraassen zurück(Van Fraassen 1966: 487) -, folgert Broome, dass Sätze wie 'Ein Studium der Ökonomie hat gleich viel Wert wie ein Studium der Rechtswissenschaft' unter einer Präzisierung wahr und unter einer anderen Präzisierung des Terminus 'hat gleich viel Wert wie' falsch sein können. In der Terminologie des Supervaluationismus ausgedrückt heisst dies, dass der Satz nicht simpliciter wahr oder nicht super-wahr ist (Keefe 2007: 162).

10 Man weiss aus der Psychologie, dass das Gegenteil der Fall ist (vgl. z. B. sogenanntes Framing). 
verhalten scheint, dass beispielsweise oft nicht Vagheit den Grund dafür darstellt, was ist es dann? Im nächsten Kapitel wird deshalb eine Typologisierung einer präferenzbasierten Sichtweise vorgenommen und das wichtigste Argument für Unvergleichbarkeit eingeführt. Das Phänomen wird genauer erfasst. 


\section{Kapitel 3}

Verschiedene

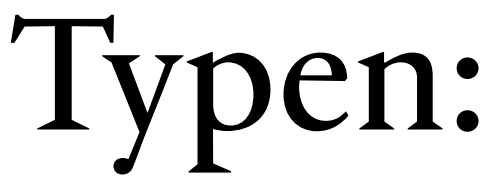

Evaluatives

Unwissen als

wichtigstes

Argument für

Unvergleich-

barkeit 
Im letzten Kapitel wurde eine präferenzbasierte Theorie von Unvergleichbarkeit eingeführt und gegen mögliche Einwände verteidigt. In diesem Kapitel wird diese Sichtweise von Unvergleichbarkeit weiter präzisiert. Dies geschieht dadurch, dass die Präferenzversion in verschiedene Typen - sogenannte harte Fälle - aufgeteilt wird. Harte Fälle, so wie ich sie verstehe, stellen spezifische Entscheidungssituationen dar. Eine Entscheidungssituation ist ein Zustand, in der eine Akteurin die Wahl zwischen mehreren Optionen hat, welche nicht gleichzeitig realisiert werden können - der Einfachheit halber auf zwei Optionen reduziert.

Worin besteht der Mehrwert einer Typologisierung von Unvergleichbarkeit? Zuerst einmal scheint dies die Sache ja komplizierter zu machen, was ceteris paribus schlecht ist. ${ }^{1}$ Der Zweck der Typologisierung ist jedoch, dass damit konkrete Alltagsphänomene genauer beschrieben werden können. Die Aufteilung in die verschiedenen harten Fälle kann als Versuch angesehen werden, einen differenzierteren Blick auf das Phänomen der Unvergleichbarkeit zu werfen. In der Unvergleichbarkeits- und Inkommensurabilitätsdebatte werden die Beispiele für Unvergleichbarkeit nämlich relativ unreflektiert 'in denselben Topf geworfen'. Selbst wenn Unvergleichbarkeit auf einen Entscheidungskontext eingegrenzt wird, sind die Exempel immer noch sehr verschieden. In den Beispielen, die etwa Chang anfügt, geht es sowohl um die Berufswahl als auch um gewöhnliche Konsumentscheidungen wie die Wahl, ob man einen Kaffee oder einen Tee trinken will (Chang 2002a). ${ }^{2}$ Mit einer präferenzbasierten Sichtweise von Unvergleichbarkeit kann dies damit erklärt werden, dass eine Akteurin in beiden Fällen keine Präferenz bilden beziehungsweise nicht angeben kann, welche Option besser ist. Die Beispiele weisen aber grosse qualitative Unterschiede auf. Durch die Einteilung in verschiedene harte Fälle wird diesem Umstand Rechnung getragen. Die Frage,

1 Einfachheit stellt gemäss Daly kein Ziel an sich dar. Er benutzt folgendes Beispiel, um diese Aussage zu belegen: Die Griechen gingen davon aus, dass es vier Elemente gibt (Erde, Luft, Wasser und Feuer). Heute unterscheidet man mehr als hundert Elemente (Daly 2010: 147). Ich würde es allerdings eher so sehen, dass Einfachheit kein Ziel darstellt, welches andere Ziele beliebig übertrumpft.

2 Streng genommen sind es gemäss Chang Beispiele, bei denen zwei Optionen weder besser noch schlechter als noch gleich gut wie die jeweilig andere Option sind. Sie interpretiert dies nicht als Unvergleichbarkeit, sondern als Parität (Kapitel 8). 
in welchen wesentlichen Merkmalen sich die Beispiele von Unvergleichbarkeit unterscheiden, ist schwierig. Die vorgeschlagene These ist die folgende: Alle Fälle beinhalten einen Wertkonflikt respektive evaluatives Unwissen. Einige Fälle sind wichtig. Andere sind es nicht. Die meisten, aber nicht alle Fälle von Unvergleichbarkeit beinhalten zusätzlich faktische Unsicherheit. ${ }^{3}$

Das Kapitel besteht aus zwei Teilen. Zuerst werden harte Fälle von drei anderen Phänomenen unterschieden. Die negative Charakterisierung hat zum Zweck, sie von den benachbarten Phänomenen abgrenzen zu können. Im zweiten Teil werden drei verschiedene Typen von harten Fällen charakterisiert. Es wird beschrieben, inwiefern sich diese Typen voneinander unterscheiden, aber auch, was sie eint. Dies stellt in dem Sinne eine positive Charakterisierung dar, als expliziert wird, was genau mit 'hart' gemeint ist und wie verschiedene Konflikte als 'hart' individuiert werden können.

\subsection{Drei benachbarte Phänomene: Abgrenzung}

- Entscheidungen können in dem Sinne hart sein, als sie psychologisch schwierig zu treffen sind. Damit sind Fälle gemeint, in denen ein Akteur weiss, was das für ihn Richtige ist, es ihm aber ausserordentlich schwer fällt, dieses zu wählen. Ein extremes Beispiel für eine psychologisch harte Entscheidung ist folgendes: Man wird von einer Schlange in den Arm gebissen. Wenn der Arm nicht amputiert wird, stirbt man. Gegeben, dass man nicht sterben will, muss der eigene Arm amputiert werden. In diesem Beispiel benötigt man unglaublich viel Überwindungskraft, um die richtige Entscheidung zu treffen. Was in diesem Fall richtig ist zu tun, ist jedoch unbestritten und klar ersichtlich. Solche Entschlüsse sind also nicht in dem Sinne schwierig, dass es keine beste Option gibt, sondern dahingehend hart, dass es sehr viel Anstrengung benötigt, die beste Option zu wählen.

Es ist denkbar, auch wenn in dieser Arbeit kein solcher Ansatz verfolgt wird, Unvergleichbarkeit in einem anderen Sinne psycho- 
logisch zu erklären. Beispielsweise können wir deshalb keine zusammenhängenden Präferenzen besitzen, etwa keine Präferenz für das Studium der Rechtswissenschaften gegenüber dem der Ökonomie haben, weil in dieser Entscheidungssituation sehr viele Faktoren berücksichtigt werden müssen und dies zu einer Überforderung und zu einer Überlastung der Aufmerksamkeitsspanne führt (Resnik 1990: 24). In unserem Beispiel heisst dies, dass bei der Studienwahl zu viele Dinge gleichzeitig evaluiert werden müssen (Interesse, spätere Berufsmöglichkeiten etc.). Gemäss diesem Ansatz kann Unvergleichbarkeit letztlich auf unsere mangelnde Fähigkeit zur Reflexion zurückgeführt werden.

Die Abgrenzung von psychologisch schwierigen Fällen meint aber nur die Abgrenzung von Fällen, in denen eine beste, aber unglaublich schwierig realisierbare Option existiert.

- $\quad$ Entscheidungen können auch deshalb hart sein, weil sie moralisch schwierig sind. Denken wir an Sartres Dilemma, in dem man sich zwischen den Optionen 'als Widerstandskämpfer für die eigene Nation kämpfen' und 'sich um die eigene Mutter kümmern' entscheiden muss (Sartre 1957). Dieses Beispiel kann in dem Sinne als moralischer Konflikt interpretiert werden, als unterschiedliche Pflichten in Konfrontation miteinander stehen: die Pflicht, die Nation zu unterstützen, versus die Pflicht, sich um die eigene Mutter zu kümmern. Moralisch schwierige Entscheidungen beschränken sich aber nicht auf konfligierende Pflichten. Sie können zum Beispiel auch Entscheidungssituationen darstellen, in denen Nutzenabwägungen und deontologische Gebote zu alternativen Handlungen führen. In dem aus der Moralphilosophie bekannten Trolley-Problem sieht man sich vor die Entscheidung gestellt, einen Menschen auf ein Zuggleis zu werfen, um fünf andere zu retten. Es gibt Philosophen, welche die Meinung vertreten, dass Inkommensurabilität respektive Unvergleichbarkeit vorwiegend in ethisch schwierigen Situationen zu finden sei (vgl. etwa Griffin 1997). Griffin spricht in diesem Zusammenhang von 'hard choices in morality' (Griffin 1997: 48). Dabei meint er Situationen, in denen man sich zwischen zwei Normen befindet, wobei keine dominant ist: '(...) we find ourselves more painfully suspended somewhere in the middle between two norms, with neither dominating.' (Griffin 1997: 51). 
Im vorliegenden Buch wird von dieser Sichtweise abgewichen. In harten Fällen können moralische Überlegungen eine Rolle spielen. Harte Fälle müssen aber nicht zwingend moralisch schwierige Entscheidungen darstellen. ${ }^{4}$

- Entscheidungen können auch in dem Sinne schwierig sein, als sie mit faktischer Unsicherheit verbunden sind. Was damit gemeint ist, kann mithilfe moderner Entscheidungstheorien wie der philosophischen Entscheidungstheorie erläutert werden (vgl. etwa Resnik 1990). Gemäss dieser Theorie beinhaltet eine Entscheidung drei Komponenten: Handlung, Zustand und Resultat. Das Resultat ('outcome') wird dabei durch die Handlung ('act') und den Zustand ('state') bestimmt. Dies kann mithilfe des folgenden Beispiels veranschaulicht werden: Ein Akteur überlegt sich, ob er nach Australien oder nach Südafrika verreisen will. Nehmen wir der Einfachheit halber an, dass der Akteur die Entscheidung ausschliesslich vom Wetter abhängig macht und zusätzlich weiss, ob gutes Wetter eintreffen wird. Das heisst, dass sich der Akteur sicher sein kann, welche Handlungen zu welchen Resultaten führen. Grafisch kann dies wie folgt veranschaulicht werden: ${ }^{5}$

\begin{tabular}{llll} 
& & \multicolumn{2}{c}{ Zustände } \\
& & Wetter gut & Wetter schlecht \\
Handlungen & Südafrika & Resultat (1) & Resultat (0) \\
& Australien & Resultat (1) & Resultat (0)
\end{tabular}

Entscheidungen können nun in dem Sinne schwierig sein, als der Akteur nicht weiss, welche Zustände und ergo welche Resultate eintreffen werden. In der Entscheidungstheorie spricht man von Entscheidungen unter Risiko oder Entscheidungen unter Unsicherheit - je nachdem, wie gut die Wahrscheinlichkeiten eingeschätzt werden können. Wir sind nun einmal nicht der Laplacesche Dämon - zum Glück

4 Ich sehe es gleich wie Levi, wenn er notiert: 'The notion of value conflict with which I am concerned has a far greater applicability than the domain of moral dilemmas.' (Levi 1986: 46).

5 Die Zahl in der Klammer entspricht jeweils der Wahrscheinlichkeit, dass Zustände und Handlungen zu diesem Resultat führen. Streng genommen ist die Wahrscheinlichkeit nie 1 beziehungsweise kann die Zahl 1 als 'beinahe 100 Prozent' interpretiert werden. Für das Resultat wird gewöhnlich ein bestimmtes Nutzenniveau eingesetzt. 
nicht - und wissen oft nicht, was nach einer getroffenen Entscheidung geschieht. Je nachdem, was geschehen wird, würden wir uns so oder anders entscheiden.

Ich denke, dass viele harte Fälle faktische Unsicherheit beinhalten. Die Abgrenzung ist schwieriger als im Falle der anderen beiden Phänomene (moralisch und psychologisch schwierige Entscheidungen). Im letzten Kapitel der Arbeit wird der Frage nachgegangen und sie bejaht, ob mit einem harten Fall, je nachdem, ob er faktische Unsicherheit beinhaltet oder nicht, anders umgegangen werden muss.

Faktische Unsicherheit spielt dementsprechend eine wichtige Rolle. Harte Fälle sind aber nicht bloss Fälle der faktischen Unsicherheit. Dafür sprechen zwei Argumente:

Das erste besteht darin, dass sich harte Fälle durch mehr faktisches Wissen nicht zwingend auflösen lassen. Wenn das Studium der Philosophie mehr Spass und das Studium der Rechtswissenschaften mehr gesellschaftliche Anerkennung bietet, lässt sich der harte Fall der Studienwahl durch zusätzliche faktische Information nicht unbedingt auflösen.

Das zweite Argument dafür ist, dass Fälle, die ausschliesslich wegen faktischen Unsicherheiten schwierig sind, in einem ganz spezifischen Sinne rational ausfallen können. Insbesondere Entscheidungen unter Risiko können mithilfe der Entscheidungstheorie rational (im Sinne einer Nutzenmaximierung) aufgelöst werden. Man kann dies so verstehen, dass sich die Optionen in eine ordinale Reihenfolge bringen lassen, beziehungsweise man kann sagen, welches die beste Option darstellt. Das Ferienbeispiel kann wie folgt modifiziert werden, um diesen Punkt zu veranschaulichen: Nehmen wir an, dass dem Akteur die gleichen Handlungen zur Auswahl stehen: nach Australien verreisen oder nach Südafrika fliegen. Der Einfachheit halber können wir annehmen, dass der Akteur die Entscheidung einerseits wiederum vom Wetter und andererseits davon abhängig macht, wie es seinen Kindern, die er auf die Reise mitnehmen will, an den jeweiligen Orten gefallen würde. Nehmen wir ebenfalls vereinfachend an, dass die Wetterverhältnisse in Australien und Südafrika die gleichen sind und mit einer Wahrscheinlichkeit von 50 Prozent davon ausgegangen werden kann, dass das Wetter gut sein wird. Die Wahrscheinlichkeit, dass Australien den Kindern gefällt, wird dabei 
vom Akteur auf 70 Prozent und im Falle von Südafrika auf 10 Prozent geschätzt. ${ }^{6}$ Damit eine Entscheidung als Entscheidung unter Risiko analysiert werden kann, muss zusätzlich angenommen werden, dass der Akteur den einzelnen Resultaten ein entsprechendes Nutzenniveau zuordnen kann, das des Akteurs subjektive Wertschätzung der einzelnen Resultate widerspiegelt. Zur Veranschaulichung wird willkürlich eine solche Präferenzordnung vorgenommen. ${ }^{7}$ Daraus ergibt sich folgende Entscheidungssituation:

\begin{tabular}{llllll} 
& & \multicolumn{4}{c}{ Zustände } \\
& & $\mathrm{sW}$ & $\mathrm{sW}$ & $\mathrm{ns} W$ & $\mathrm{~ns} W$ \\
Handlungen & $\mathrm{Kgn}$ & $\mathrm{Kg}$ & $\mathrm{Kgn}$ \\
& Australien & $70(0.35)$ & $0(0.15)$ & $15(0.35)$ & $0(0.15)$ \\
& Südafrika & $30(0.45)$ & $5(0.05)$ & $25(0.45)$ & $3(0.05)$
\end{tabular}

Es gilt: $70(0.35)+15(0.35)>30(0.45)+5(0.05)+25(0.45)+3(0.05)$

Das heisst, dass der erwartete Nutzen der Reise nach Australien höher ausfällt. Der Akteur kann aufgrund einer Präferenz entscheiden: Australien stellt die bessere Option als Südafrika dar. Ob er das Richtige tut, weiss der Akteur nicht. Dies hängt davon ab, welche Zustände eintreffen werden. Bei allgemein schlechtem Wetter wäre Südafrika zum Beispiel die bessere Option. 'Wetter gut + Kindern gefällt es nicht', 'Wetter schlecht + Kindern gefällt es', 'Wetter schlecht + Kindern gefällt es nicht'. Daraus ergeben sich unterschiedliche Wahrscheinlichkeiten für die einzelnen Zustände. Die Wahrscheinlichkeit des Zustands, dass das Wetter schön ist und Australien den Kindern gefällt, liegt zum Beispiel bei 35 Prozent (0.5 multipliziert mit 0.7). In diesem Zusammenhang muss berücksichtigt werden, dass der Zustand 'Kindern gefällt es' davon abhängig ist, ob der Akteur nach Südafrika oder Australien verreist. Wir haben es also nicht mit absoluten Wahrscheinlichkeiten der Zustände, sondern mit Zustandswahrscheinlichkeiten zu tun, welche konditional von den Handlungen abhängen.

7 Australien + schönes Wetter + Kindern gefällt es $(70)>$ Südafrika + schönes Wetter + Kindern gefällt es (30) > Südafrika + schlechtes Wetter + Kindern gefällt es (25) > Australien + schlechtes Wetter + Kindern gefällt es (15) > Südafrika + schönes Wetter + Kindern gefällt es nicht (5) > Südafrika + schlechtes Wetter + Kindern gefällt es nicht (3) > Australien + schönes Wetter + Kindern gefällt es nicht $(0)>$ Australien + schlechtes Wetter + Kindern gefällt es nicht (0) (die Zahl in der Klammer steht jeweils für das Nutzenniveau). 
Nach diesen Abgrenzungen zu benachbarten Phänomenen - im obigen Beispiel: faktische Unsicherheit - geht es im nächsten Teil des Kapitels nun um eine positive Charakterisierung von Unvergleichbarkeit.

\subsection{Verschiedene Typen harter Fälle}

Die Idee besteht darin, die positive Charakterisierung mithilfe verschiedener Kriterien vorzunehmen. Genauer stellen die in dieser Arbeit relevanten harten Fälle eine Kombination von drei Kriterien dar: Wertkonflikt, faktische Unsicherheit und Wichtigkeit. Um zu überprüfen, welcher Typ harter Fall vorliegt, können folgende Fragen gestellt werden: (1) Ist bei der Entscheidung ein Wertkonflikt involviert? (2) Ist die Entscheidung mit faktischer Unsicherheit verbunden? (3) Ist die Entscheidung wichtig? Daraus ergeben sich drei verschiedene Typen von Unvergleichbarkeit: ${ }^{8}$ Im Folgenden werden zuerst die Kriterien erläutert und anschliessend - je nachdem, wie Kriterien kombiniert werden - drei Typen von Unvergleichbarkeit beschrieben.

\section{Wertkonflikt}

Unvergleichbarkeit sollte primär über Wertkonflikte charakterisiert werden. Primär meint, dass jeder harte Fall einen Wertkonflikt beinhaltet. Hingegen ist nicht jeder harte Fall wichtig oder mit faktischer Unsicherheit verbunden. Das wichtigste Argument für Unvergleichbarkeit, so die These, sind Wertkonflikte. Man kann auch sagen, dass die Unvergleichbarkeit von Optionen auf die Unvergleichbarkeit von Werten zurückgeführt werden kann.

Was ist Wert-Unvergleichbarkeit? Es ist wesentlich einfacher zu sagen, was Wert-Unvergleichbarkeit nicht ist. Abgrenzend betrachtet, darf Wert-Unvergleichbarkeit nicht mit Wertepluralismus, was Chang 'diversity of values' nennt, gleichgesetzt werden (Chang 1997: 14). Das

8 Rein kombinatorisch gesehen ergeben sich wohlgemerkt mehr Möglichkeiten. Es werden nur diejenigen Typen erwähnt, welche (i) in unserem Alltag eine wichtige Rolle zu spielen scheinen und/oder (ii) dazu führen können, dass eine Akteurin Optionen nicht eindeutig als besser/schlechter/gleich gut bewerten kann. 
heisst auch, dass Wert-Vergleichbarkeit nicht mit Wertmonismus identifiziert werden kann.

Das Konzept des Wertepluralismus wird sehr unterschiedlich begriffen. Oft wird darunter eine metaphysische These verstanden, also eine These darüber, welche Art von Dingen es gibt, die Wert besitzen. Wertepluralismus meint demnach, dass mehrere Werte existieren, die nicht unter einem sogenannten 'supervalue' subsumiert werden können (vgl. Chang 2015b). Wertmonismus hingegen besagt, dass sämtliche Werte auf einen einzigen reduziert werden können: 'According to monism, all values ultimately reduce to a supervalue.' (Chang 1997: 16). Eine Reduktion kann so verstanden werden, dass, wenn ein Wert gegegeben ist, dann automatisch auch der andere Wert gegeben ist (und umgekehrt): 'One value reduces to another if there is nothing more to having the one than having the other. For example, the beauty in a painting reduces to pleasure if there is nothing more to the painting's being beautiful than its providing pleasure to those who view it.' (Chang 2013: 3). Oft ist mit einem Wert in diesem Zusammenhang aber nicht etwas wie Schönheit, sondern eine moralische Qualität gemeint. In diesem Buch wird Wertepluralismus allerdings weder als ontologisches noch als ein ausschliesslich moralisches Konzept verstanden. In Anlehnung an Nagel ist mit Wertepluralismus gemeint, dass es verschiedene Typen von Werten gibt. Einerseits spielen dabei auch nicht-moralische Werte eine Rolle. Anderseits ist es zweitrangig, ob eine ontologische Reduktion möglich oder unmöglich ist. Gemäss Nagel lassen sich die verschiedenen Dinge, die uns wichtig sind, anhand verschiedener Werttypen systematisieren. In seinem Aufsatz 'The Fragmentation of Value' vertritt er die These, dass es fünf grundlegende Typen von Werten gibt: 1 Spezifische Pflichten - zum Beispiel Pflichten gegenüber der eigenen Familie. 2 Allgemeine Rechte - zum Beispiel das Recht auf freie Meinungsäusserung. 3 Nutzen - jegliche Aspekte von Freude und Leid sämtlicher Personen oder fühlender Wesen. 4 Perfektionistische oder intrinsische Ziele - zum Beispiel die Entdeckung eines neuen Planeten. 5 Persönliche Ziele - zum Beispiel eine Weltreise machen (Nagel 1979: 129, 130). Inwiefern die Systematisierung beziehungsweise die detaillierte Aufteilung in die verschiedenen Bereiche plausibel ist, soll nicht diskutiert werden. Entscheidend ist, dass Wertepluralismus meint, dass es verschiedene Typen von Werten gibt, die irreduzibel sind. 
Der Wertepluralismus, auf diese Weise verstanden, ist jedoch keine hinreichende Bedingung für Wert-Unvergleichbarkeit. ${ }^{9}$ Betrachten wird hierzu zwei Beispiele:

Stocker geht davon aus, und dies ist plausibel, dass viele, wenn nicht beinahe alle unsere Entscheidungen plurale Erwägungen beinhalten (Stocker 1990: 180). Er erwähnt in diesem Zusammenhang folgendes Beispiel: Man überlegt sich, ob man die Stadt zu Fuss, mit dem Auto, mit dem Bus oder mit dem Taxi durchqueren will. Dabei können folgende Dinge eine Rolle spielen: Das Auto ist schneller, aber der Bus billiger; mit dem Auto muss nach einem Parkplatz gesucht werden, aber mit dem Bus kann man nicht genau dorthin fahren, wohin man will; etc. In diesem Beispiel sind nach Stocker plurale Werte wie Zeit, Geld, Sicherheit oder Freiheit involviert (Stocker 1990: 178).

Oder nehmen wir an, dass es sowohl einen Werttyp der 'wirtschaftlichen Freiheit' als auch einen Werttyp der 'Gleichheit' gibt. Nehmen wir weiter an, die Option $x$ führe zu mehr Umverteilung und zu mehr Gleichheit und die Option $y$ zu weniger Umverteilung und mehr wirtschaftlicher Freiheit. $x$ stellt also eine Option dar, die den Wert 'Gleichheit' fördert, während $y$ eine Möglichkeit darstellt, die den Wert 'wirtschaftliche Freiheit' unterstützt. $x$, die konkrete Handlungsoption, könnte etwa für eine Erhöhung, und $y$, die andere Handlungsoption, für eine Senkung des Unternehmenssteuersatzes stehen. Falls der Wertepluralismus eine hinreichende Bedingung für Wert-Unvergleichbarkeit wäre, würde folgen, dass die Werte in obigen darstellt, ist schwieriger zu beantworten. Ich denke, dass er auch keine notwendige Bedingung darstellt, weil es selbst in einer Welt, in welcher der Wertmonismus wahr wäre, Unvergleichbarkeit geben könnte. Nehmen wir beispielsweise an, dass wir nur an Steigerung von Lust und Verminderung von Unlust interessiert wären. Es scheint sich so zu verhalten, dass es selbst dann noch Unvergleichbarkeit geben könnte. Die Art und Weise, wie man etwa beim Hockeyspielen Lust empfindet, ist derart verschieden von der Lust, die einem das Biertrinken mit Freunden bereitet, dass z. B. die Entscheidung, ob man dreimal pro Woche ins Hockeytraining gehen oder mit Freunden Bier trinken will, durchaus einen Fall von Unvergleichbarkeit darstellen kann - auch wenn die Steigerung von Lust den einzigen Wert darstellt. Ich setze voraus, dass unterschiedliche Lüste keinen Wertepluralismus implizieren. Dies kann bestritten werden. Es kann eingewendet werden, dass Lust selbst inhärent plural ist; deshalb, weil extrem unterschiedliche Formen des positiven Erlebens existieren. Man könnte argumentieren, dass kein einheitliches Ding namens Lust existiert, welches empirisch messbar ist. 
beiden Beispielen unvergleichbar sind. Ich verstehe dies so, dass die Werte in keine ordinale Reihenfolge gebracht werden können. Dies wiederum würde nahelegen, dass auch die Handlungsoptionen, die diese Werte befördern, nicht ordinal geordnet werden können.

Das folgt aber nicht unbedingt. Schliesslich ist eine ordinale Reihung zwischen pluralen Werten möglich. So könnte die betreffende Person sehr wohl zu dem Ergebnis gelangen, dass der Wert der 'wirtschaftlichen Freiheit' weniger wichtig ist als der Wert der 'Gleichheit', und eine ordinale Reihung dieser pluralen Werte vornehmen. Dieser Befund wird auch in Stockers Beispiel deutlich. Obwohl verschiedene Werte im Spiel sind, kann ein Akteur unter Umständen ohne Probleme eine eindeutige Bewertung der Entscheidungssituation vornehmen, zum Beispiel die Fahrt mit dem Bus präferieren.

Wenn Wert-Unvergleichbarkeit nicht mit Wertepluralismus gleichgesetzt werden kann, wie soll sie dann verstanden werden? Am plausibelsten scheint anzunehmen, dass die verschiedenen Typen von Werten nicht ordinal geordnet werden können. Die Frage ist, was erfüllt sein muss, damit dies der Fall ist. Anders gefragt: Wertepluralismus + was führt dazu, dass sich eine Akteurin mit unvergleichbaren Werten konfrontiert sieht? Eine Möglichkeit besteht darin, dass dies genau dann der Fall ist, wenn die Akteurin schlicht nicht bereit ist, die verschiedenen Werte gegeneinander abzuwägen (vgl. Wolf 2005). Nicht-Bereitschaft ${ }^{10}$ und Unmöglichkeit fallen bei einer subjektivistischen Deutung von Unvergleichbarkeit zusammen: Wenn wir nicht bereit sind, die Werte in einem harten Fall gegeneinander abzuwägen, dann ist es auch nicht möglich, die Werte in eine Rangordnung zu bringen. Und ergo können auch die Optionen, die diese unterschiedlichen Werte befördern, nicht als besser oder schlechter als oder als gleich gut wie die jeweilig andere Option bewertet werden. Wenn eine Akteurin nicht bereit ist, eine spezifische Pflicht - zum Beispiel, sich um die Mutter zu kümmern - einem persönlichen Ziel - zum Beispiel, eine Weltreise zu machen - gegenüberzustellen, um Nagels Systematisierung von Werten zu verwenden, befindet sie sich in einem harten Fall, der auf einen Wertkonflikt zurückgeführt werden kann. 
Eine andere Möglichkeit besteht darin, dass die Rangierung unabhängig von unserer Bereitschaft unmöglich ist. Deshalb, weil die Werte an sich nicht vergleichbar sind. In diesem Fall können spezifische Pflichten und persönliche Ziele grundsätzlich in keine Rangordnung gebracht werden.

Aus der deliberativen Perspektive des Akteurs macht es dabei wohl keinen wesentlichen Unterschied, ob die erste oder die zweite Möglichkeit plausibel ist (ich komme gleich darauf zurück). Weil es aber relativ schwierig zu sagen scheint, weshalb gewisse Werte per se nicht ordinal geordnet werden können, wird von der ersten Deutung ausgegangen.

Weshalb ist die These problematisch, dass Werte per se in keine Rangordnung gebracht werden können? Einen überzeugenden Grund nennt Chang. Sogenannte nominal-notable Vergleiche sind gemäss Chang immer möglich. Damit sind mögliche Vergleiche zwischen unterschiedlichen Extremen gemeint. Viel 'wirtschaftliche Freiheit' ist beispielsweise vergleichbar mit respektive besser als nur ganz wenig 'Gleichheit'. Daraus folgt, dass pluralistische Werte nicht per se unvergleichbar sein können. Ein weiterer Grund ist, dass es sich um Entscheidungen und somit um die Perspektive des Akteurs handelt. Im Kontext von Entscheidungen geht es dabei nicht um eine Wahl zwischen zwei universellen Werten, sondern um diejenige zwischen zwei Instanzen dieser Werte. Anders formuliert vergleicht man bei der Berufswahl zum Beispiel nicht den allgemeinen Wert der Kreativität und den allgemeinen Wert der ökonomischen Sicherheit, sondern inwiefern konkrete Instanzen davon zu einem gelungenen Leben beisteuern (vgl. Betzler 1999).

Die Frage ist nun, was es genau heisst, nicht bereit zu sein, Werte beziehungsweise konkrete Instanzen dieser Werte gegeneinander abzuwägen. Gemäss Wolf kann die mangelnde Bereitschaft, verschiedene Werte gegeneinander abzuwägen, damit erklärt werden, dass wir sie als nicht austauschbar, unersetzbar und unbezahlbar empfinden (Wolf 2005: 16). Dies würde bedeuten, dass einem die konkreten Instanzen dieser Werte allesamt wichtig sind. Es könnte aber auch sein, dass man nicht weiss, wie hoch man die unterschiedlichen Dinge wertschätzt. In diesem Zusammenhang könnte eingewendet werden, dass es im obigen Beispiel um eine Pflicht geht und es bei einer 
solchen keinen Sinn macht, davon zu sprechen, ob und wie sehr man etwas wertschätzt. Der Kern einer Pflicht, so der potenzielle Einwand, besteht nämlich genau darin, dass man nicht abwägen soll - ganz nach dem Motto: Es ist eine Pflicht. Punkt! Denken wir deshalb an ein anderes Exempel, bei dem keine offensichtlichen Pflichten im Spiel sind, zum Beispiel an die im letzten Kapitel erwähnte Wahl, ob man in Bern oder Basel leben möchte.

Im letzten Kapitel wurde davon ausgegangen, dass der Gegenstand der Deliberation die Gründe sind, welche für Bern oder Basel sprechen. In diesem Fall sind es die diesen Gründen zugrundeliegenden Werte, welche in den unterschiedlichen Optionen realisiert werden. So kann man sich vorstellen, dass es für die Akteurin einen Wert hat, in Bern zu wohnen, weil ihr Freund in Bern lebt, und einen Wert, in Basel zu wohnen, weil dies näher am Arbeitsplatz liegt. Dass der Akteurin beides - der Wert sozialer Beziehungen und der Wert des effizienten Arbeitens - wichtig ist, wird im Beispiel vorausgesetzt. Die plausiblere Deutung ist, dass die Akteurin deshalb nicht bereit ist, die beiden Werte gegeneinander abzuwägen, weil sie nicht weiss, wie sehr sie die beiden wertschätzt. Inwiefern die beiden Werte dabei per se oder nur für die Akteurin unvergleichbar sind, spielt aus einer deliberierenden Perspektive keine entscheidende Rolle. Im einen Fall denkt die Akteurin, dass die Werte an sich unvergleichbar sind. Im anderen Fall überlegt sie, dass die Werte für sie unvergleichbar sind. Ich will nicht behaupten, dass dies überhaupt keinen Unterschied macht. Im zweiten Fall ist die Unvergleichbarkeit in einem gewissen Sinne selbst verschuldet. Die Akteurin könnte also die geografische Nähe zum Freund als wichtiger ansehen und die Unvergleichbarkeit auflösen. Ich werde diesen Punkt im letzten Kapitel der Arbeit wieder aufnehmen. An dieser Stelle kann festgehalten werden, dass dies eine theoretische Möglichkeit darstellt, welche mit der Phänomenologie nicht übereinstimmt: Wenn die Akteurin nicht weiss, wie hoch sie es wertschätzt, am gleichen Ort wie der Freund zu wohnen, kann sie nicht auf Knopfdruck oder durch zusätzliche Überlegungen eine gegenteilige Wertschätzung erlangen. Weshalb nicht? Das Argument, das ich im letzten Kapitel dafür anfügen werde, geht vereinfacht gesagt davon aus, dass die Auflösung einer evaluativen Unwissenheit Zeit braucht und nur durch Erfahrung erfolgen kann. 
Man kann also sagen, dass es sich dabei um evaluatives oder normatives Unwissen oder evaluative Ignoranz handelt. Was ist damit gemeint? Beginnen wir damit, was es nicht bedeutet.

Der Begriff des normativen Unwissens ist ein technischer Begriff, der verschiedene Bedeutungen besitzt. Eine Bedeutung - und das ist nicht gemeint - lautet, dass man nicht weiss, welche moralischen Normen oder welche ethischen Theorien richtig sind. ${ }^{11}$ Gemäss diesem Verständnis meint normatives Unwissen, dass man etwa zu ca. 40 Prozent einen akt-utilitaristischen und zu 60 Prozent einen deontologischen Ansatz für ethisch richtig hält. ${ }^{12}$ Eine andere Bedeutung - das ist ebenfalls nicht gemeint - lautet, dass sich die Unwissenheit nicht aufTheorien, sondern auf konkrete Handlungen bezieht. Zum Beispiel, dass man einerseits glaubt, es sei besser, einen Menschen zu töten, um hundert andere Menschen zu retten, und andererseits, es sei besser, keinen Menschen zu töten, um hundert andere Leben zu retten.

Ich meine eine Unwissenheit über Werte oder Gründe, die nicht zwingend mit Ethik zu tun hat. Die evaluative oder normative Unwissenheit meint allgemeiner, dass man nicht weiss, wie Fakten bewertet werden sollen. Beispielsweise weiss ein Akteur nicht, wie der Fakt, dass es leicht regnet, zu bewerten ist.

Die Unwissenheit ist also insofern evaluativ, als sie sich weder auf Fakten (Regnet es? Wohnt die Freundin tatsächlich in Bern?) noch darauf bezieht, was passieren wird (Wird es regnen? Bleibt die Freundin in Bern?), sondern darauf, welche Gründe von Fakten generiert werden. Stellt leichter Regen einen Grund dar, einen Regenschirm einzupacken, oder stellt es keinen Grund dafür dar? ${ }^{\text {13 }}$

In der Dissertation Normative Uncertainty von William MacAskill, welche 2014 an der Universität in Oxford eingereicht worden ist, wird der Begriff des normativen Unwissens so verwendet. wie z. B., dass man die Chance minimiert, moralisch falsch zu handeln. Diese Lösung wird in Lockhart 2000 vertreten.

In Sepielli 2009 wird der Begriff des normativen Unwissens auf diese Weise verwendet. Sepielli geht dabei der Frage nach, ob es allgemeinere Prinzipien gibt, die im Falle normativer Unwissenheit angewendet werden können. Er bejaht die Frage und präsentiert eine theoretisch interessante Lösung, welche allerdings kardinale Vergleichbarkeit voraussetzt. Weil dies m. E. eine zu starke und unrealistische Annahme ist, wird darauf im letzten Kapitel der Arbeit, wenn es um die Frage geht, wie mit unvergleichbaren Optionen umgegangen werden soll, nicht eingegangen. 
Anders als in der Entscheidungstheorie bezieht sich die Unwissenheit somit nicht auf die Zustände in der Welt. Es geht nicht darum, dass man nicht weiss, welche Resultate durch die Handlungen hervorgebracht werden. Die Unwissenheit betrifft den Wert der Optionen selbst. Das heisst, dass sich die Unwissenheit auf etwas bezieht, was die Entscheidungstheorie als gegeben annimmt. Sowohl Entscheidungen unter Risiko (man kennt die Wahrscheinlichkeiten der Resultate) als auch Entscheidungen unter Unwissenheit (man braucht die Wahrscheinlichkeiten der Resultate nicht zu kennen) setzen voraus, dass ein Akteur die Güte der einzelnen Resultate beurteilen kann. Im Falle einer evaluativen Unwissenheit hingegen ist der Akteur nicht fähig, ihnen einen eindeutigen Wert zuzuordnen.

In dieser Hinsicht besteht eine Parallele mit Pauls aktuell viel diskutiertem Ansatz der sogenannten transformativen Erfahrung auch hierbei kann den Optionen kein eindeutiger Wert zugeschrieben werden. Vereinfacht gesagt ist mit transformativer Erfahrung gemeint, dass bestimmte Ereignisse wie zum Beispiel die Geburt des eigenen Kindes einen zu einer ganz anderen Person machen können, und man schlicht nicht weiss, welche Person das sein wird (Paul 2014). Paul argumentiert, dass die Entscheidung, das erste Kind zu bekommen, in wesentlichen Aspekten gleich ist, wie die Wahl, ob man ein Mensch bleiben oder ein Vampir werden will (Paul 2014: 82). In beiden Fällen weiss man nicht, wie sich die eigenen Präferenzen verändern werden und wie sich die neue Erfahrung anfühlen wird. In beiden Fällen kann dasjenige, was passieren wird, nicht aufgrund von ähnlichen und bereits gemachten Erfahrungen bewertet werden (Paul 2014: 43).

Sollten harte Fälle dementsprechend nicht anhand des Konzepts der transformativen Erfahrung charakterisiert werden? Ich denke nicht. Es ist in diesem Zusammenhang wichtig zu sehen, dass Paul in ihrer Argumentation erstens folgende Prämisse vorauszusetzen scheint: Das Hineinfühlen in das zukünftige Selbst - Wie wird es sich für mich anfühlen, Vater zu sein? - ist der wichtigste Teil im Deliberationsprozess. Explizit argumentiert Paul weiter, dass das Hineinfühlen misslingt. Man ist völlig ahnungslos, wie es sich anfühlen wird. Unabhängig davon, welche Evidenz herbeigezogen wird, sei es beispielsweise die Lektüre psychologischer Studien (wissenschaftliche Verallgemeinerungen) oder das Hineinfühlen in Menschen, die 
bereits Kinder haben (Empathie), sie bietet keine Anhaltspunkte. Sehr vereinfacht gesagt, folgert Paul aus diesen beiden Prämissen, dass eine Entscheidung, die transformative Erfahrung beinhaltet, nicht rational ist (vgl. auch Paul 2015). Das bedeutet, dass die Entscheidung gemäss Paul dann nicht rational ist, wenn sie auf Erwartungen basiert, wie sich die transformative Erfahrung anfühlen wird. Ich habe hingegen dafür argumentiert, dass transformative Entscheidungen sehr wohl rational sein können (vgl. Reuter und Messerli 2018). Es ist fragwürdig, inwiefern die 'Wie-wird-es-sich-anfühlen-Komponente' der relevante Umstand für die Entscheidung ist (vgl. hierzu auch Messerli (Manuskript)). Die Studienwahl scheint nicht deshalb ein Problem darzustellen, weil man nicht weiss, wie es sich anfühlen wird, Philosophiestudent zu sein. Trotzdem ist es wohl richtig, dass phänomenale Einfühlung beim Vergleich von Optionen manchmal eine Rolle spielt und ohne direkte Erfahrung misslingt. Somit kann transformative Erfahrung - genauer: die mangelnde Fähigkeit, sich in eine Situation hineinzuversetzen - eine Entscheidung zusätzlich komplex machen. Grundsätzlich ist es jedoch plausibler, harte Fälle anhand von evaluativem Unwissen zu charakterisieren: Die Studienwahl ist vor allem deshalb schwierig, weil man nicht weiss, wie wichtig einem verschiedene Werte respektive konkrete Instanzen dieser Werte sind, die in den Optionen unterschiedlich realisiert werden.

Zusammenfassend kann festgehalten werden, dass ein Wertkonflikt meint, dass man nicht in der Lage ist, Werte abzuwägen. Dies wiederum kann so interpretiert werden, dass man nicht weiss, wie sehr man die unterschiedlichen Werte schätzt.

Ich denke, dass die Kaffee-Tee-Beispiele von Unvergleichbarkeit damit allerdings nicht hinreichend von den interessanteren Fällen wie der Berufswahl abgegrenzt werden können. Man könnte argumentieren, dass selbst bei der Wahl zwischen Kaffee und Tee unterschiedliche Werte im Spiel sind und der Akteur unter Umständen nicht weiss, wie wichtig ihm diese sind. Je mehr man darüber weiss, wie die Produkte produziert und transportiert worden sind etc., stellt dies unter Umständen gar keinen so abwegigen Gedanken dar, ganz nach dem Motto: Je mehr faktisches Wissen, desto mehr Unwissenheit darüber, was einem wichtig ist. Auf jeden Fall scheint es sinnvoll zu sein, neben dem Kriterium des evaluativen Unwissens zusätzliche Merkmale 
heranzuziehen, um eine Typologisierung der verschiedenen harten Fälle vornehmen zu können.

\section{Wichtigkeit und faktische Unsicherheit}

Ein weiteres Kriterium besteht darin, ob die Entscheidung wichtig oder unwichtig ist. Ist eine Entscheidung wichtig für das eigene Leben, bedeutet dies meiner Ansicht nach, dass damit viele neue Optionen ermöglicht oder verunmöglicht werden. Ob man Anwalt oder Musiker werden will, ist gemäss dieser Betrachtung ein wichtiger Entschluss, weil man als Anwalt viele Dinge tun kann, die man als Musiker nicht praktizieren kann, und viele Dinge nicht tun kann, die man als Musiker ausüben kann. Ob man lieber Kaffee oder Tee will, ist gemäss dieser Betrachtung ein unwichtiger Entschluss, weil man sich in der Regel durch diese Entscheidung keine neuen Optionen schafft oder solche verhindert.

Wichtigkeit auf obige Weise verstanden ist weder eine hinreichende noch eine notwendige Bedingung für Unvergleichbarkeit. Es gibt sowohl Fälle von Unvergleichbarkeit, die nicht wichtig sind, als auch wichtige Entscheidungen zwischen vergleichbaren Optionen. Trotzdem sind einige Fälle von Unvergleichbarkeit wichtig und andere nicht. Welche Fälle dies sind, sagt obiges Kriterium nicht. Es ist inhaltsneutral formuliert.

Man könnte denn auch einwenden, dass das Kaffee-Tee-Beispiel auch mithilfe dieses Kriteriums nicht von der Berufswahl unterschieden werden kann und es Menschen gibt, für welche die Wahl zwischen Kaffee und Tee eine wichtige Entscheidung darstellt. Ich denke allerdings, dass die vorgeschlagene Differenzierung zwischen wichtigen und unwichtigen Entscheidungen eine plausible Unterscheidung darstellt, bei der letztlich jeder für sich beurteilen muss, welche Wahl ihm welche neuen Möglichkeiten öffnet oder schliesst. Für die meisten Menschen stellt die Auslese zwischen Kaffee und Tee keine wichtige Entscheidung dar. Man wählt heute Kaffee und kann morgen weiterleben, als hätte man Tee getrunken. Immer noch ist es möglich, Anwalt zu werden oder Martina zu heiraten. Man kann sich dies auch so vorstellen, dass der Aufwand wesentlich höher ist, eine wichtige Entscheidung zu korrigieren. Wenn man realisiert, dass das 
Studium der Jurisprudenz die falsche Wahl war, dann ist der Aufwand, diesen Entschluss zu korrigieren, in der Regel hoch. Beispielsweise muss man dann eine andere Ausbildung absolvieren.

Gemäss diesem Kriterium stellt die umgangssprachliche Redeweise 'Jede Entscheidung ist wichtig' also quasi per definitionem eine Dummheit dar, da nicht jede Entscheidung weitreichende Konsequenzen hat.

Ich habe wohlgemerkt nicht den Anspruch, an dieser Stelle eine Theorie der Wichtigkeit von Entscheidungen vorzulegen. Was soll der Begriff der Wichtigkeit leisten? Es geht darum, dass damit konkrete Beispiele, die in der Literatur für Unvergleichbarkeit angefügt werden, in verschiedene Beispieltypen eingeteilt werden können. Es liegt meiner Meinung nach auf der Hand, dass die Studienwahl in dem Sinne wichtiger als die Entscheidung zwischen Kaffee und Tee ist, als sie weiterreichende Konsequenzen hat. Der Teufel steckt dann wie immer im Detail: Kann die Wichtigkeit beispielsweise tatsächlich rein quantitativ verstanden werden, in $\mathrm{dm}$ Sinne, dass eine wichtige Entscheidung mehr Optionen öffnet und schliesst oder die Korrektur einer wichtigen im Gegensatz zu jener einer unwichtigen Entscheidung mehr Aufwand erfordert?

Das andere Kriterium besteht darin, ob die Entscheidung faktische Unsicherheit beinhaltet oder nicht. In der Tendenz beinhalten wichtige - im Gegensatz zu unwichtigen - Entscheidungen faktische Unsicherheit. Dies gilt jedoch nicht immer, wie wir gleich sehen werden.

Mithilfe dieser drei Kriterien - Wertkonflikt, Wichtigkeit und faktische Unsicherheit - können nun die drei Typen von Unvergleichbarkeit beziehungsweise drei harte Fälle unterschieden werden.

\section{Drei Typen von Unvergleichbarkeit}

Typ1: Wertkonflikt + faktisch unsicher + wichtig

Dieser Typ beinhaltet sowohl einen Wertkonflikt als auch faktische Unsicherheit. Zusätzlich werden durch die Entscheidung viele neue Optionen ermöglicht respektive verunmöglicht. Obwohl es immer auch von der Akteurin abhängig ist, mit welchem Typ sie 
konfrontiert ist, kann die Berufswahl ein Beispiel für einen solchen Fall darstellen. Das heisst, dass (i) die Akteurin nicht weiss, wie wichtig ihr die Werte sind, die in den verschiedenen Berufen unterschiedlich realisiert werden, (ii) faktische Unsicherheit eine zentrale Rolle spielt und (iii) die Entscheidung viele neue Optionen öffnet respektive schliesst. So kann man sich vorstellen, dass im Anwaltsberuf finanzielle Sicherheit und im Musikerberuf Spass verwirklicht wird, obwohl offenbar auch Anwältinnen Freude an der Arbeit haben können und man nicht weiss, wie wichtig einem dies ist. Dazu kommt, dass die Entscheidung zusätzlich wichtig ist und sich in besonderer Weise durch faktische Unsicherheit auszeichnet. Dies liegt daran, dass die Akteurin nicht genau weiss, wie sich die zu wählenden Optionen entwickeln werden. So könnte sich etwa im Fall der Klarinettistin die öffentliche Subventionspolitik derart verändern, dass kaum noch Stellen für Musikerinnen verfügbar sind.

\section{Typ2: Wertkonflikt + wichtig}

Dieser Typ harter Fall ist wichtig, beinhaltet einen Wertkonflikt, aber keine faktische Unsicherheit. Die Entscheidung, ob man dreimal pro Woche ins Hockeytraining gehen will oder nicht, kann als solcher Fall angesehen werden. Durch die fixen Trainingszeiten und zusätzlichen Meisterschaftsspiele am Wochenende werden viele andere Optionen verunmöglicht, etwa das Biertrinken mit Freunden. In diesem Beispiel sind etliche unterschiedliche Werte im Spiel. So ist man durch das regelmässige Training körperlich und auch geistig fitter, aber weniger frei etc. Die faktische Unsicherheit kann in diesem Fall dadurch ausgeschlossen werden, dass die Konsequenzen der Handlung relativ gut abgeschätzt werden können: Man versucht nicht, einen Puck ins Tor zu schiessen, sondern bestellt im Restaurant ein Bier.

\section{Typ3: Wertkonflikt}

Das Kaffee-Tee-Beispiel, sofern man dieses wie Chang als Beispiel für Unvergleichbarkeit ansehen will, gehört in diese Kategorie. Als Exempel für einen solchen Fall kann aber auch die Entscheidung angesehen werden, ob man im Restaurant das leckere und ungesunde oder das gesunde und weniger leckere Menü bestellen will. Eine Person, welche in einem Speiselokal Essen bestellt, kann sich sehr sicher 
sein, dass sie auch dasjenige bekommt, was sie bestellt hat (deshalb keine faktische Unsicherheit).

Bevor der Frage nachgegangen wird, was die unterschiedlichen Typen gemein haben, möchte ich zuerst auf zwei Einwände eingehen und anschliessend darlegen, inwiefern sich die drei Fälle hinsichtlich 'Härte' unterscheiden.

Der eine Einwand besagt, dass Typ 3 und Typ 2 in dem Sinne unplausibel sind, als bei jeder Entscheidung faktische Unsicherheit involviert ist. Der andere Einwand bestreitet, dass Unvergleichbarkeit als evaluatives Unwissen charakterisiert werden kann. Gemäss Chang kann sich ein Akteur in einem harten Fall nämlich sicher sein, dass er sich bezüglich seines Urteils - zwei Optionen sind weder besser noch schlechter als noch gleich gut wie die jeweilig andere Option - nicht täuscht. ${ }^{14}$ Chang meint damit, dass ein Akteur genügend Evidenz besitzt, dass die Optionen nicht besser/schlechter/gleich gut sind. Wir können dies auf zwei Weisen interpretieren: (1) Der Akteur weiss, wie hoch er die verschiedenen Werte beziehungsweise Optionen wertschätzt. (2) Harte Fälle verschwänden auch dann nicht, wenn der Akteur hinreichend über die Konsequenzen der Entscheidung informiert wäre. Chang würde vermutlich sagen, dass beide Punkte richtig sind. Ich denke, dass der erste Punkt falsch und der zweite richtig ist.

Beginnen wir mit dem zweiten Punkt, nämlich damit, dass Unvergleichbarkeit nicht unbedingt faktische Unsicherheit beinhalten muss. Schaber erwähnt in diesem Zusammenhang das Beispiel einer Person, die sich zwischen verschiedenen Berufen - Leiter eines wissenschaftlichen Forschungsprojekts versus Beamtenstelle in der Universitätsleitung - entscheiden muss, und er argumentiert, dass die Person selbst dann noch unfähig ist, den Wert der beiden Berufe zu vergleichen, wenn sie ausreichend über die Konsequenzen ihrer Entscheidung informiert ist. Diese Person, so Schaber, weiss trotzdem nicht, wofür sie sich entscheiden soll, weil sie nicht in der Lage ist, unterschiedliche Werte wie Autonomie, Wissen, emotionale Sicherheit und sozialen Status zu ordnen (Schaber 1994: 157). Auch wenn 
gerade die Berufswahl eher dem ersten Typus eines harten Falles entspricht und dabei in der Regel faktische Unsicherheiten im Spiel sind, ist der generelle Punkt plausibel, dass nicht jeder harte Fall faktische Unsicherheit involviert. Ich interpretiere dies nicht wie Schaber, dass der Akteur vollständig informiert ist, sondern so, dass er sicher genug sein kann, die relevanten Werte oder Gründe erfasst zu haben. Anders formuliert heisst dies, dass sich ein Akteur manchmal sicher sein kann, dass er alles berücksichtigt hat, was für die Entscheidung relevant ist. Zusätzliche faktische Informationen können daran nichts ändern, der Wertkonflikt würde bestehen bleiben. Dieser Einwand trifft ergo nicht zu.

Hinsichtlich des ersten Punktes würde Chang vermutlich davon ausgehen, dass die Person in Schabers Beispiel weiss, wie wichtig ihr Autonomie, Wissen und sozialer Status sind. Ich denke, dass die plausiblere Interpretation aber darin besteht, dass die Person dies nicht weiss und sich die Optionen deshalb in keine ordinale Reihenfolge bringen lassen. Ich habe kein gutes Argument für diese These und kann lediglich ein Gedankenexperiment anfügen: Nehmen wir an, dass Chang Recht hat und die Person weiss, wie wichtig ihr die verschiedenen Werte sind. Nehmen wir zusätzlich an, dass die Werte gewisse Kohärenzbedingungen wie Transitivität erfüllen. Damit meine ich, dass zum Beispiel keine intransitiven Wertrelationen der Form 'Autonomie ist wichtiger als sozialer Status und sozialer Status wichtiger als Wissen und Wissen wichtiger als Autonomie' vorliegen. In dieser Situation, so meine ich, könnte die Akteurin einen Beruf ceteris paribus als besser bewerten. Der Umstand, dass die Optionen auch in diesem Fall nicht als besser oder schlechter als oder als gleich gut wie die jeweilig andere Option bewertet werden könnten, müsste durch die Verletzung von Kohärenzbedingungen verständlich gemacht werden. Anders schiene dies nicht erklärbar zu sein, da die Akteurin ja sonst weiss, wie wichtig ihr die in den Optionen zum Ausdruck kommenden Werte sind - ich schliesse an dieser Stelle mögliche psychologische Erklärungen wie Überlastung der Aufmerksamkeitsspanne aus.

Welche wesentlichen Unterschiede weisen die verschiedenen Typen harter Fälle in puncto 'Härte' auf?

Der Umgang mit demjenigen Typ, der sowohl einen Wertkonflikt als auch faktische Unsicherheit beinhaltet und zusätzlich eine 
wichtige Entscheidung darstellt, stellt in dem Sinne den 'härtesten' Fall dar, als es sich bei diesem Typus um eine Entscheidung handelt, die längere Zeiträume betrifft, die den Akteur selbst unterschiedlich prägen. Das heisst, dass es sich um Entscheidungen zwischen langfristigen, komplexen Optionen handelt.

Dies trifft auch auf den zweiten Typus zu. Faktische Unwissenheit macht die Entscheidung aber zusätzlich schwierig, schlicht deshalb, weil zusätzliche faktische Informationen unter Umständen einen Unterschied in der Evaluation ausmachen können (vgl. Kapitel 10).

Am wenigsten 'hart' ist der dritte Typus. In Anlehnung an Andreou 2011 kann bei diesen harten Fällen davon ausgegangen werden, dass sie sich zwar nicht unbedingt punktuell, auf der Ebene einer einzelnen Entscheidung, aber auf einer 'höheren Ebene' auflösen lassen. Damit ist gemeint, dass ein Akteur Pläne und Strategien entwickeln kann, in denen den relevanten Werten Rechnung getragen wird, wie zum Beispiel, dass manchmal das leckere und manchmal das gesunde Menü gewählt wird. Bei diesen harten Fällen kann ein Akteur versuchen, über einen längeren Zeitraum hinweg eine gute 'Balance' zu finden (sofern er das will). Der Umstand, dass eine einzelne Entscheidung aufgrund der Unvergleichbarkeit der Werte (Genuss versus Gesundheit) nicht rational ist (dies wird natürlich in den folgenden Kapiteln noch gezeigt werden müssen), erweist sich dann als nicht so 'schlimm', da die allgemeine Strategie - weder völlig puritanisch noch völlig ungesund leben - sehr wohl mehr oder weniger begründet ausfallen kann: Nur gesund ist auch krank.

\section{Gemeinsamkeit}

Alle drei Fälle stellen Wertkonflikte dar. Alle drei unterschiedlichen Typen von harten Fällen, so die These, können als Unvergleichbarkeit gedeutet werden - in dem Sinne, dass sich die Optionen zum Zeitpunkt der Wahl nicht eindeutig als besser/schlechter/gleich gut bewerten lassen. Das heisst, dass der Begriff harter Fall (egal welcher Typus) auf eine sehr allgemeine Eigenschaft von Entscheidungssituationen referiert. Diese Eigenschaft besteht darin, dass ein Akteur keine Präferenz formulieren kann. Im Konkreten können die Entscheidungen durch evaluatives Unwissen charakterisiert werden, 
wobei die Wichtigkeit der Entscheidung und der Grad der faktischen Unsicherheit berücksichtigt werden müssen.

Wenn harte Fälle auf diese Weise charakterisiert werden, nimmt man im Grunde genommen eine Abgrenzung vor. Das heisst, dass man mit diesem Begriff eine Grenze zieht: nämlich zwischen sogenannten harten und nicht harten Fällen. Nicht harte Fälle sind - im Gegensatz zu harten Fällen - Entscheidungssituationen, in denen wir eine eindeutige Bewertung der Handlungsoption vornehmen können, eine Handlungsoption bevorzugen oder indifferent zwischen den Optionen sind.

\subsection{Zusammenfassung}

In diesem Kapitel wurde die Präferenzversion von Unvergleichbarkeit in verschiedene harte Fälle aufgeteilt.

Im ersten Teil des Kapitels wurde argumentiert, dass harte Fälle von moralisch, psychologisch oder faktisch schwierigen Fällen abgegrenzt werden müssen.

Im zweiten Teil des Kapitels wurde dafür plädiert, dass sich die verschiedenen Typen von Unvergleichbarkeit anhand dreier wesentlicher Kriterien charakterisieren lassen: Der einfachste harte Fall beinhaltet einen Wertkonflikt. Bei einem Wertkonflikt können Werte nicht gegeneinander abgewogen werden, was so verstanden werden kann, dass man nicht weiss, wie hoch man die unterschiedlichen Werte schätzt (evaluatives Unwissen). Der zweite harte Fall enthält einen Wertkonflikt und stellt zusätzlich eine wichtige Wahl dar. Dies kann so interpretiert werden, dass damit viele neue Optionen ermöglicht respektive verunmöglicht werden. Der dritte und schwierigste harte Fall stellt einen Wertkonflikt dar, ist wichtig und zusätzlich mit faktischer Unsicherheit verbunden. Dies kann so ausgelegt werden, dass man zusätzlich nicht weiss, was nach der getroffenen Entscheidung passieren wird, und dies unter Umständen für die Evaluation relevant ist.

In diesem Kapitel wurde somit die These aufgestellt, dass alle Fälle von Unvergleichbarkeit auf evaluatives Unwissen zurückgeführt werden können und einige wichtig und/oder mit faktischer 
Unsicherheit verbunden sind. Unabhängig davon, von welchem Typ ausgegangen wird, kann eine Akteurin dabei keine Präferenz bilden. Gegen diese These kann allerdings immer noch eingewendet werden, dass überhaupt keine solchen Entscheidungssituationen existieren. Davon handelt das nächste Kapitel. 


\section{Kapitel 4}

Argumente

gegen

Unvergleich-

barkeit:

Zurückweisung 
Im letzten Kapitel wurden verschiedene Typen von Unvergleichbarkeit (harte Fälle) unterschieden. In allen besitzt die Akteurin weder eine Präferenz noch ist sie indifferent zwischen den zur Wahl stehenden Optionen. In diesem Kapitel betritt der Skeptiker die Bühne. Dieser bestreitet, dass es Unvergleichbarkeit gibt - eine Möglichkeit, die ernst genommen werden muss. Es ist nämlich denkbar, dass man mit Unvergleichbarkeit $a$ meint, $a$ aber keine Entsprechung in der Welt hat. Das bedeutet nicht, dass es 'die Welt' nicht gibt. Unser Skeptiker ist weniger radikal. Gemeint ist die Möglichkeit, dass die im zweiten Kapitel erläuterte Definition eine leere Extensionsmenge aufweist. In diesem Kapitel verfolge ich das Ziel, diese Möglichkeit auszuschliessen. Ich plädiere dafür, dass die Argumente, welche die skeptische Position stützen (ein Akteur besitzt stets eine Präferenz oder ist indifferent), nicht plausibel sind und es überzeugende Gründe für Unvergleichbarkeit gibt. Grundsätzlich sollte der Skeptiker bereits überzeugt worden sein. Ich habe im letzten Kapitel gezeigt, dass evaluatives Unwissen ein starkes - meiner Meinung nach das wichtigste - Argument für Unvergleichbarkeit darstellt: Ein Akteur weiss nicht, wie hoch er unterschiedlichen Werte schätzt, und kann deshalb keine Präferenz bilden. Dennoch kann der Skeptiker immer noch zwei wichtige Argumente gegen Unvergleichbarkeit vorbringen. In diesem Kapitel werden beide Einwände pariert und zwei weitere Argumente für Unvergleichbarkeit erläutert. Der Stellenwert dieses kurzen Kapitels liegt darin, mögliche verbleibende Zweifel an der These - Unvergleichbarkeit stellt ein reales Phänomen dar - auszuräumen.

Das Kapitel gliedert in zwei Teile: Im ersten Teil werden zwei Argumente gegen Unvergleichbarkeit zurückgewiesen. Im zweiten Teil werden zwei weitere Argumente für Unvergleichbarkeit eingeführt.

\subsection{Einwände}

Eine These, in diesem Fall, dass es keine Unvergleichbarkeit gibt, sollte in der Regel, sofern sie nicht selbstevident ist, begründet werden. Es ist nicht selbstevident, dass es keine Unvergleichbarkeit gibt. Also muss die These begründet werden. Ich sehe in erster Linie 
zwei Möglichkeiten, wie der Skeptiker argumentieren könnte, dass Unvergleichbarkeit kein reales Phänomen darstellt. Die erste besteht in einem Verweis auf die Entscheidungstheorie. Die zweite basiert auf einem sogenannten Revealed-preference-Ansatz. Beide Argumentationen können nicht überzeugen. Beginnen wir mit dem Verweis auf die Axiomatik moderner Entscheidungstheorien.

\section{Axiomatik der Entscheidungstheorie}

Entscheidungstheorien gehen davon aus, dass keine harten Fälle existieren. Dies folgt aus der Axiomatik dieser Theorien. Entscheidungstheorien sind axiomatisch nämlich so aufgebaut, dass Handlungsoptionen in einer spezifischen Ordnung stehen beziehungsweise sie sich stets in eine Rangordnung bringen lassen. ${ }^{1}$ Am Beispiel der philosophischen Entscheidungstheorie kann dies präzise wie folgt dargestellt werden:

$(\forall x)(\forall y)((x P y \vee y P x \vee x I y) \wedge(x P y \rightarrow \neg x I y) \wedge(y P x \rightarrow \neg x I y) \wedge(x I y \rightarrow(\neg x P y \wedge \neg y P x))$

' $P$ ' steht für eine Präferenz, ' $I$ ' steht für Indifferenz, ' $x$ ' und ' $y$ ' repräsentieren die Optionen. Der erste Teil obiger Konjunktion bildet das Vollständigkeitsaxiom (connectedness-axiom), und die restlichen drei Teile stellen die Asymmetrieaxiome der philosophischen Entscheidungstheorie dar (Resnik 1990). Was obige Formel besagt, ist, dass man, vor die Wahl zwischen zwei Optionen gestellt, stets eine Alternative bevorzugt oder indifferent zwischen ihnen ist und nicht gleichzeitig verschiedene Einstellungen einnimmt - also zum Beispiel nicht gleichzeitig $x$ gegenüber $y$ präferiert und indifferent zwischen $x$ und $y$ ist. In der Terminologie eines entscheidungstheoretischen Modells wird dies manchmal auch als Vollständigkeit der Präferenzrelation, als completeness axiom oder als Ordnungsaxiom bezeichnet. Dieses Ordnungsaxiom erinnert an Arithmetik, genauer an ein Axiom für reelle Zahlen, das besagt, dass eine Zahl entweder grösser oder kleiner als oder gleich gross wie eine andere Zahl sein muss; eine vierte Möglichkeit gibt es nicht.

1 Unter einer Rangordnung muss dabei eine ordinale und keine kardinale Reihung verstanden werden (vgl. Kapitel 1). 
Die Frage, ob harte Fälle existieren oder nicht, stellt aber eine substanzielle These dar. Es gibt keine Notwendigkeit für die Existenz oder Nicht-Existenz harter Fälle. Nur weil das Verhältnis von Zahlen einer bestimmten Axiomatik folgt, bedeutet nämlich nicht, dass sich die Dinge in einem praktischen Kontext analog verhalten. Mit der bestehenden Axiomatik moderner Entscheidungstheorien kann man die Nicht-Existenz harter Fälle nicht begründen.

Es ist wichtig zu sehen, dass es in diesem Zusammenhang nicht darum geht, weshalb das Vollständigkeitsaxiom gelten soll. Sowohl das Vollständigkeitsaxiom als auch die restlichen Axiome ${ }^{2}$ der philosophischen Entscheidungstheorie stellen sicher, dass konkrete Optionen in eine Rangordnung gebracht werden können (deshalb sollen die Axiome gelten). Es geht darum, dass die philosophische Entscheidungstheorie, und dies gilt auch für andere Entscheidungsmodelle wie etwa die mikroökonomische Präferenztheorie, voraussetzt, dass wir diese Rangierung auch vornehmen können.

Das heisst, dass man argumentieren müsste, weshalb wir stets eine solche Rangordnung der Alternativen vornehmen können. Dass die Vollständigkeit der Präferenzrelation stets gilt, wird in der Entscheidungstheorie aber ohne Argumentation vorausgesetzt. Die Entscheidungstheorie setzt voraus, dass sich Optionen stets vergleichen lassen. Sie liefert selbst aber kein Kriterium, weshalb dies der Fall ist. Es stellt eine Annahme dar, deren Plausibilität mithilfe der Entscheidungstheorie selbst nicht getestet werden kann. Die Präferenzen betreffend Resultate sind im Gegensatz zu den Präferenzen betreffend Handlungen exogen gegeben. Das heisst, sie werden nicht im Modell bestimmt.

Genauer besteht das Problem darin, dass die Entscheidungstheorie nicht abbilden kann, dass Präferenzen manchmal auch konstruiert werden müssen. ${ }^{3}$ Die Entscheidungstheorie kann nicht einfangen,

2 Resnik baut sein formales Entscheidungsmodell auf acht Axiomen auf. Ich habe die ersten vier Axiome oben dargestellt und zusammengenommen als Vollständigkeit der Präferenzrelation bezeichnet. Die restlichen vier Axiome stellen Transitivitätsbedingungen dar. Sie lauten wie folgt: $x P y \wedge y P z \rightarrow x P z ; x P y \wedge x I z \rightarrow z P y ; x P y \wedge y I z \rightarrow x P z ; x I y \wedge y I z \rightarrow x I z$.

3 Ich orientiere mich hierbei an der Kritik, welche auch von einigen Psychologen geltend gemacht wird: '(...) Although utility theory does not address psychological processes per se, the completeness principle implies that preferences are activated or retrieved, as opposed to being calculated, at the time of choice.' (Warren, McGraw und Van Boven 2011: 194). 
dass diese Konstruktion unter Umständen nicht erfolgreich ist und man keine Präferenz formulieren kann, wobei die Redeweise 'wird konstruiert' die Abgrenzung zu 'ist gegeben' meint. In entscheidungstheoretischen Modellen wird davon ausgegangen, dass eine Akteurin mit bestimmten Präferenzen ausgestattet ist. Weil diese Präferenzen als gegeben angesehen werden können, lassen sich sämtliche Entscheidungsprobleme aufösen: Entweder hat man eine Präferenz für Ökonomie oder eine für Rechtswissenschaft. Die Idee, dass Präferenzen konstruiert werden müssen, geht vom Gegenteil aus: Man besitzt (noch) keine Präferenz für Ökonomie oder Rechtswissenschaft und muss überlegen, welcher Option man welchen Wert zuordnen will.

\section{Theorie aufgedeckter Präferenzen}

Auch aus der Theorie aufgedeckter Präferenzen (revealed-preference) folgt, dass es keine Unvergleichbarkeit gibt. Dieses Argument ist wesentlich ernster zu nehmen als der blosse Verweis auf einen Formalismus.

Vereinfacht gesagt, geht man in dieser Theorie von folgender These aus: Ein Akteur präferiert $x$ gegenüber $y$ genau dann, wenn er (i) $x$ wählt und (ii) $y$ auch erhältlich ist.

Innerhalb der Unvergleichbarkeits- und Inkommensurabilitätsdebatte vertritt Andreou einen solchen Ansatz. Andreou hat den Vorschlag gemacht, dass harte Fälle mit der Entscheidungstheorie kompatibel sind und deshalb gar kein Problem für die praktische Rationalität darstellen (Andreou 2005). Auch wenn $x$ weder besser noch schlechter noch gleich gut wie $y$ ist, kann man die Optionen - 'for the purpose of choice' - trotzdem ordnen (Andreou 2005: 259). Andreou geht davon aus, dass ein Akteur in der Lage ist, Handlungsoption $x$ qua Entscheidung als besser einzustufen. Es liegt nahe, dies so zu interpretieren, dass sich Werte nicht von Präferenzen und Präferenzen nicht von Entscheidungen unterscheiden. In letzterem Fall sind Handlungsoptionen dann eo ipso unmöglich unvergleichbar (Schaber 1994: 160): Die gewählte Option ist stets die beste, da sich die Güte einer Option dadurch zeigt, dass sie gewählt wird.

Die zugrunde liegende Motivation der Theorie aufgedeckter Präferenzen besteht im Wesentlichen darin, Präferenzen für eine 
wissenschaftliche Beobachterin messbar zu machen. Wählen wir einen introspektiven Zugang (die Perspektive des Akteurs), kommt zuerst die Präferenz und dann die Wahl. Diese Reihenfolge wird gerade umgekehrt. Die Wissenschaftlerin beobachtet zuerst eine Wahl und schliesst auf eine Präferenz (Sen 1986). Die Präferenzen einer Person werden durch ihr Wahlverhalten aufgedeckt (Sellmaier 2007: 39, meine Hervorhebung). Man kann sich vorstellen, dass sich in einem sonst leeren Kühlschrank zwei Joghurts befinden: ein Himbeer- und ein Schokoladenjoghurt. Wenn eine Akteurin nun das Schokoladenjoghurt wählt, folgt gemäss dieser Theorie, dass sie dieses lieber als das Himbeerjoghurt mag. In diesem Zusammenhang sind aber etliche Zusatzannahmen nötig, wie etwa, dass man nicht unüberlegt, uninformiert oder auf der Basis falscher Überzeugungen entscheidet oder von der Mutter gezwungen wird, das Himbeerjoghurt zu wählen. Die weiterführende Frage, unter welchen Bedingungen das Wahlverhalten irrational ist, spielt für uns keine Rolle: Führt beispielsweise das Hinzukommen eines Vanillejoghurts dazu, dass man anstatt das Himbeernun das Schokoladenjoghurt wählt? Falls ja, ist das Wahlverhalten gemäss gängiger Auffassung irrational. ${ }^{4}$ Wichtig ist, dass grundsätzlich mindestens drei verschiedene Auslegungen der Theorie aufgedeckter Präferenzen unterschieden werden können (vgl. Hausman 2012):

Erstens: Mit Revealed-preference-Ansatz kann gemeint sein, dass eine Entscheidung Evidenz liefert, welche Präferenzen ein Akteur besitzt. Dies ist mit ziemlicher Sicherheit richtig und sollte unstrittig sein. Wenn eine Akteurin argumentiert, dass Steuerehrlichkeit wichtig ist und selbst Steuern hinterzieht, findet sie diese wohl gar nicht so wichtig. Diese Interpretation, welche nicht ausschliesst, dass Optionen unvergleichbar sein können, stösst nicht nur in der Ökonomie auf Zuspruch. In Language of Morals schreibt Hare: 'If we were to ask of a person What are his moral principles? the way in which we could be most sure of a true answer would be by studying what he did (...) it would be when (...) he was faced with choices or decisions between alternative courses of action, between alternative answers to the question What shall I do?, that he would reveal in what principles of conduct he really believed.' (Hare 1972: 1). Ich interpretiere Hare so,

4 Genauer wird in diesem Fall die sogenannte Bedingung Beta verletzt, welche Sen als Kriterium einer kontextfreien Auswahlfunktion einführt (vgl. Sen 1970). 
dass konkrete Entscheidungen und Handlungen einen guten Indikator dafür darstellen, was ein Akteur tatsächlich denkt.

Zweitens: Mit Revealed-preference-Ansatz kann, wie zu Beginn dargestellt, gemeint sein, dass Präferenzen über konkrete Entscheidungen (ein Akteur wählt $x$ ) definiert werden. Dies ist falsch. Dies würde etwa bedeuten, dass ein Akteur keine Präferenz besitzt, wenn er keine Wahl trifft, was schlicht unwahr sein kann. Beispielsweise kann man sich weigern zu wählen. Es ist aber auch dann falsch, wenn die Theorie nur auf diejenigen Alternativen angewendet wird, zwischen denen ein Akteur tatsächlich wählt. Der einfachste Grund dafür ist, dass Präferenzen eine Wahl nur via Überzeugung beeinflussen: Romeos Wahl zu sterben basiert auf der falschen Überzeugung, dass Julia auch tot ist. Die Wahl zeigt nicht, welche Präferenz Romeo besitzt.

Drittens: Mit Revealed-preference-Ansatz kann gemeint sein, dass Präferenzen über hypothetische Entscheidungen (ein Akteur würde $x$ wählen) definiert werden. Auf diese Interpretation, die mit der ursprünglichen Idee bricht, dass es sich beim Ansatz der aufgedeckten Präferenzen um eine rein empirische Theorie handelt, wird nicht weiter eingegangen. Für uns ist wichtig festzuhalten, dass Unvergleichbarkeit nur dann ausgeschlossen wird, wenn Präferenzen über Entscheidungen definiert werden.

Gegen diese Interpretation der Theorie aufgedeckter Präferenzen muss - abgesehen von den obig genannten Problemen - im Kontext von Unvergleichbarkeit eingewendet werden, dass ein Unterschied existiert zwischen 'Eine Akteurin tut $x$ ' und (2) 'Eine Akteurin tut $x$, weil sie denkt, dass $x$ die beste Wahl darstellt'. Relevant ist die Frage, welche Option man wählen will oder soll, und nicht, welche man tatsächlich realisiert. Entscheiden tut man sich in der Regel so oder so. Eine Wahl kann aber nicht von sich aus zeigen, welche Präferenzen eine Akteurin besitzt. Oder anders formuliert: Eine Entscheidung lässt die Rationalität des Entschlusses nicht nachvollziehen. Dies führt natürlich zur Frage, was man sich unter einer rationalen Wahl genau vorzustellen hat. Darauf wird erst im übernächsten Kapitel eingegangen. Vereinfachend kann an dieser Stelle folgendes Argument angefügt werden, weshalb eine Entscheidung nicht zwingend etwas mit Rationalität zu tun hat: 
1. Rationalität hat oft damit zu tun, in welchem Verhältnis Optionen zueinander stehen (auch wenn Vergleichbarkeit nicht notwendig für Rationalität ist).

2. Im Falle von Unvergleichbarkeit besteht kein positives Verhältnis zwischen den Alternativen.

$\therefore$ Im Falle von Unvergleichbarkeit ist keine rationale Entscheidung möglich.

Obige Prämissen werden in Kapitel 6 präzisiert und erläutert. Intuitiv lässt sich das Argument anhand des folgenden Beispiels veranschaulichen: Ein Akteur muss entscheiden, welcher Bewerber eine offene Stelle bekommen soll. Nehmen wir an, dass noch zwei gute und unterschiedliche Kandidaten im Rennen sind, und der Akteur nicht angeben kann, für welchen Kandidaten die besseren Gründe sprechen. Dementsprechend hat er keine begründete Präferenz zugunsten eines Bewerbers, entscheidet sich aber dennoch für Bewerber 1. In diesem Fall ist die Wahl nicht deshalb rational, weil er sich entschieden hat. Der Akteur hat willkürlich eine Ordnung vorgenommen. Das hat nichts mit einer Begründung zu tun. Die Entscheidung wäre rational, wenn mehr Gründe dafür sprächen, Bewerber 1 anstatt Bewerber 2 einzustellen, und der Akteur deshalb das Urteil fällt, dass der erste Kandidat den Job bekommen soll. In diesem Beispiel hat eine rationale Entscheidung damit zu tun, in welchem Verhältnis die Optionen zueinander stehen. Und dies wiederum hat mit der Präferenz des Akteurs zu tun, wobei die Idee darin besteht, dass sie aufgrund von Argumenten pro und contra die jeweiligen Optionen gebildet wird.

\subsection{Weitere Argumente für Unvergleichbarkeit}

In der philosophischen Diskussion besteht weitgehend Konsens darüber, dass viele Fälle existieren, in denen man weder sagen kann, welche Option besser oder schlechter ist, noch, ob die Optionen gleich gut sind. Chang schreibt etwa: '(...) it is plausible that such judgements are very common.' (Chang 2002a: 671).

Das wichtigste Argument, weshalb dies richtig ist und es harte Fälle gibt, wurde im letzten Kapitel entwickelt: Evaluatives Unwissen 
stellt, wie zu Beginn erwähnt, das stärkste Argument für Unvergleichbarkeit dar. Im Folgenden werden zwei weitere Argumente für Unvergleichbarkeit genannt:

Erstens wird die Existenz harter Fälle von der phänomenologischen Evidenz gestützt: Wir machen in unserem Alltag die Erfahrung, dass wir Optionen manchmal nicht als besser, schlechter oder gleich gut bewerten können. ${ }^{5}$ Dies wird begleitet von Gefühlen des Zweifels und der Unsicherheit bezüglich solcher Entscheidungen.

Weder solche Zweifel noch der Verweis auf die phänomenologische Evidenz können wohlgemerkt genügen, um die Existenz von Unvergleichbarkeit zu begründen. Es gibt wissenschaftstheoretisch gesehen einen grossen Unterschied zwischen 'Ich finde $a$ plausibel' und 'Es ist empirisch belegt, dass $a$ '. Was man plausibel findet, könnte falsch sein. In der philosophischen Diskussion ist bislang keine empirische Bestätigung für die Existenz von Unvergleichbarkeit erfolgt, geschweige denn auf empirische Studien hingewiesen worden. Eine unmittelbare empirische Bestätigung ist aber auch nicht möglich, da sich Präferenzen, etwa im Gegensatz zu konkreten Handlungen, nicht direkt beobachten lassen. ${ }^{6}$

Hinzu kommt, dass wir uns auch nach einer getroffenen Entscheidung zwischen solchen Optionen manchmal schlecht fühlen, sogar, wenn wir keine Gründe dafür angeben können, weshalb die getroffene Entscheidung falsch war. So reagieren wir darauf beispielsweise häufig mit Bedauern. Das Bedauern ist dabei insofern speziell, als die Entscheidung nicht zwingend rückgängig gemacht werden will. Normalerweise verhält sich dies anders. Wenn man eine Entscheidung bedauert, will man sie in der Regel korrigieren (Elster 1984: 68).

Die These, dass Optionen manchmal nicht eindeutig als besser/ schlechter/gleich gut bewertet werden können, wird zudem dadurch gestützt, dass wir in harten Fällen auch nicht mit Indifferenz reagieren, was jedoch der Fall wäre, wenn wir die Optionen tatsächlich für gleich gut hielten. Es ist uns nicht egal, weil die Entscheidung (i) einen 
Wertkonflikt respektive evaluatives Unwissen beinhaltet und (ii) zusätzlich wichtig sein kann. ${ }^{7}$ Beide Arten von Einstellungen scheinen dafür zu sprechen, dass die relevanten Optionen nicht hinreichend in eine Ordnung gebracht werden können.

Auch dabei gilt, dass aus der Tatsache, dass wir vor und nach solchen Entscheidungen manchmal mit Zweifel und Bedauern reagieren, nicht unbedingt folgt, dass die jeweiligen Optionen nicht als besser/ schlechter/gleich gut bewertet werden können. Das Objekt des Bedauerns mag zum Beispiel einfach der Tatsache geschuldet sein, dass nicht beide Optionen gewählt werden konnten (am liebsten beides). Dies sollte dementsprechend ebenfalls als zusätzliche Evidenz und nicht als Knock-down-Argument für die Existenz von Unvergleichbarkeit gedeutet werden.

Es geht darum, dass Gefühle wie Bedauern ein Anzeichen dafür darstellen, dass die Optionen in keine Ordnung gebracht werden können. Inwiefern das Bedauern nach der getroffenen Entscheidung in einer Konfliktsituation beziehungsweise im Falle von Unvergleichbarkeit auch rational sein kann, wäre eine weiterführende Frage.

Als zweites Argument ist das sogenannte Argument der kleinen Verbesserung zu nennen, das die Existenz von Unvergleichbarkeit stützt (vgl. z. B. De Sousa 1974). Das Argument basiert auf der intuitiven Idee, dass eine Option, welche hypothetisch ein wenig verbessert wird $(x+)$, immer besser als die ursprüngliche Option $(x)$, aber nicht besser als eine völlig verschiedene Option $(y)$ ist. Um bei einem bekannten Beispiel zu bleiben, hiesse dies, dass der Anwaltberuf mit einem um 500 Euro erhöhten Monatseinkommen $(x+)$ besser ist als der Anwaltberuf ohne diese Gehaltserhöhung $(x)$. Gleichwohl, so die Schlussfolgerung, macht ihn diese Erhöhung nicht besser als den Musikerberuf $(y){ }^{8}$ Dies lässt sich folgendermassen rekonstruieren:

7 Vgl. das letzte Kapitel. Die präzise Abgrenzung zum Phänomen der Indifferenz erfolgt jedoch erst im nächsten Kapitel.

8 Im Falle einer Gleichheit hingegen ist $(x+)$ besser als die ursprüngliche Option $(x)$ und besser als die verschiedene Option $(y)$. Ein Heuhaufen plus 50 Rappen $(x+)$ ist besser als der ursprüngliche Heuhaufen $(x)$ und ceteris paribus besser als ein anderer Heuhaufen $(y)$. 
1. Der Anwaltberuf $(x)$ ist weder besser noch schlechter als der Musikerberuf $(y)$.

2. Der Anwaltberuf mit der Gehaltserhöhung $(x+)$ ist besser als der Anwaltberuf $(x)$.

3. Der Anwaltberuf mit der Gehaltserhöhung $(x+)$ ist nicht besser als der Musikerberuf $(y)$.

$\therefore$ Der Anwaltberuf $(x)$ ist weder besser noch schlechter und auch nicht gleich gut wie der Musikerberuf $(y)$.

Grundsätzlich gilt das Argument der kleinen Verbesserung in der philosophischen Literatur als überzeugend - auch wenn daraus unterschiedliche Schlussfolgerungen gezogen werden. Meistens soll anhand dieses Arguments gezeigt werden, dass Handlungsoptionen unvergleichbar sein können (vgl. etwa Raz 1986).

Die Frage, ob dieses Argument plausibel ist, wird im nächsten Kapitel diskutiert. Ich werde argumentieren, dass das Argument der kleinen Verbesserung tatsächlich ein Argument für Unvergleichbarkeit darstellt, in dem Sinne, dass es zeigt, dass im Falle unvergleichbarer Optionen nicht die Relation der Indifferenz instantiiert ist.

Wir können daher festhalten, dass das erste Argument zusätzliche Evidenz darstellt, dass Optionen manchmal nicht als besser/ schlechter/gleich gut bewertet werden können.

Das zweite Argument - dies wird im nächsten Kapitel gezeigt werden müssen - sollte den potenziellen Skeptiker überzeugen, dass unvergleichbare Optionen nicht gleich gut sind respektive Unvergleichbarkeit nicht dasselbe wie Indifferenz ist.

\subsection{Zusammenfassung}

Im ersten Teil des Kapitels wurde argumentiert, dass zwei mögliche Argumente gegen Unvergleichbarkeit nicht plausibel sind. Weder der Verweis auf die Axiomatik der Entscheidungstheorie noch die Theorie aufgedeckter Präferenzen können überzeugen. Die entscheidungstheoretische Axiomatik setzt Vergleichbarkeit ohne Argumentation voraus. Der Hauptgrund, weshalb der Ansatz aufgedeckter Präferenzen im Kontext von Unvergleichbarkeit nicht plausibel ist, 
besteht darin, dass es einen Unterschied gibt zwischen dem, was man tut, und demjenigen, was man begründet tut. Eine Akteurin kann sich für ein Studium der Ökonomie entscheiden, auch wenn sie nicht denkt, dass es das bessere Studium als dasjenige der Rechtswissenschaft ist. Im zweiten Teil des Kapitels wurden zwei neue Argumente für die Existenz von Unvergleichbarkeit eingeführt: phänomenologische Evidenz und Gefühle wie Bedauern und Zweifel und das Argument der kleinen Verbesserung.

Bislang wurde dabei vor allem der Frage nachgegangen, weshalb eine Akteurin keine Präferenz formulieren kann. Die Plausibilität der These, dass Unvergleichbarkeit ein reales Phänomen darstellt, hängt allerdings auch damit zusammen, dass hinreichend nachvollziehbar ist, weshalb ein Akteur nicht indifferent zwischen unvergleichbaren Optionen ist. Im nächsten Kapitel geht es deshalb um die Abgrenzung zum Phänomen der Indifferenz. 


\section{Kapitel 5}

Unvergleich-

barkeit,

(In)Transitivität

und Gleich-

gültigkeit 
Im letzten Kapitel wurden zwei Argumente gegen Unvergleichbarkeit zurückgewiesen und zwei neue Argumente für Unvergleichbarkeit eingeführt. In diesem Kapitel wird eine dieser Begründungen, das sogenannte Argument der kleinen Verbesserung, im Detail analysiert. Mit diesem Argument kann gezeigt werden, dass Unvergleichbarkeit nicht mit Gleichgültigkeit verwechselt werden darf.

Dabei verfolge ich ein positives und ein negatives Argumentationsziel:

Positiv betrachtet, zeige ich, dass die im Argument der kleinen Verbesserung implizierte Intransitivität Evidenz liefert, dass Unvergleichbarkeit nicht dasselbe wie Indifferenz ist. Unvergleichbarkeit zeichnet sich durch eine spezifische Form der Intransitivität aus. Gleichgültigkeit hingegen charakterisiert sich durch eine spezifische Form der Transitivität. Das heisst, dass sich Unvergleichbarkeit und Indifferenz in diesem Merkmal unterscheiden. Inwiefern sich das Phänomen der Unvergleichbarkeit und dasjenige der Indifferenz in anderen Eigenschaften unterscheiden, ist nicht Gegenstand dieses Kapitels. Es könnten andere Gründe genannt werden, weshalb Unvergleichbarkeit nicht dasselbe wie Indifferenz ist. Mir geht es aber nicht um weitere Argumente für die Abwesenheit von Gleichgültigkeit. Um zu zeigen, dass Unvergleichbarkeit nicht dasselbe wie Indifferenz ist, ist es ausreichend, dass sie in einem wichtigen Merkmal nicht identisch sind.

Negativ betrachtet, begründe ich, weshalb die bestehende Literatur präzisiert werden muss. Einerseits gibt es Philosophen, die sagen, dass mit dem Argument der kleinen Verbesserung die Intransitivität bestimmter Konzepte belegt werden kann. Raz schreibt: 'The test of incommensurability is failure of transitivity.' (Raz 1986: 325). Hsieh geht in Anlehnung an Raz ebenfalls davon aus, dass sich Unvergleichbarkeit durch fehlende Transitivität auszeichnet (Hsieh 2005: 201). Chang sagt in einem kürzlich erschienenen Interview, dass die entscheidende Differenz zwischen Parität und Indifferenz darin bestehe, dass Parität nicht transitiv sei (Chang: 'The existentialist of hard choices', S. 6). Dabei benutzen sie alle dasselbe Argument, das Argument der kleinen Verbesserung, um obige Aussagen zu belegen. Dies ist in dem Sinne zu ungenau, als Formalisierungen des Arguments existieren, die Transitivität involvieren. Andererseits gehen 
einige Philosophen davon aus, dass Transitivität eine oder die entscheidende Prämisse im Argument der kleinen Verbesserung darstellt (z. B. Gustafsson und Espinoza 2010). Dies ist dem Sinne falsch, als Formalisierungen des Arguments existieren, die keine Transitivität involvieren. Kurz gesagt: Die Rolle der (In)Transitivität im Argument ist wesentlich komplizierter, als dies bislang in der Literatur angenommen wurde. Meiner Meinung nach ist Intransitivität ein notwendiger Bestandteil des Arguments. Transitivität hingegen kann, muss aber nicht Teil des Arguments sein. ${ }^{1}$

Wie die spezifischen Formen der Transitivität und Intransitivität genau zu charakterisieren sind und was sie für das bessere Verständnis von Unvergleichbarkeit beitragen, wird in diesem Kapitel gezeigt.

\subsection{Das Argument der kleinen Verbesserung}

Raz erwähnt zwei Strategien, wie man Inkommensurabilität beziehungsweise Unvergleichbarkeit - er verwendet die Begriffe synonym - feststellen kann (Raz 1986).

Gemäss der ersten Strategie bildet man ein Argument mit der folgenden Form:

1. $x$ ist weder besser noch schlechter als $y$.

2. $z$ ist besser als $x$.

3. $z$ ist nicht besser als $y$.

$\therefore \quad x$ ist weder besser noch schlechter als noch gleich gut wie $y$.

Ein Beispiel:

1. Eine Karriere als Anwalt ist weder besser noch schlechter als eine Karriere als Musiker. auch Intransitivität, hat den Anschein, eine Kontradiktion zu sein. Es ist deshalb wichtig zu erwähnen, weshalb dies nicht der Fall ist. Der Grund dafür ist, dass unterschiedliche Prinzipien der Transitivität gemeint sind. 
2. Eine Karriere als Feuerwehrmann ist besser als eine Karriere als Anwalt.

3. Eine Karriere als Feuerwehrmann ist nicht besser als eine Karriere als Musiker.

$\therefore$ Eine Karriere als Anwalt ist weder besser noch schlechter als noch gleich gut wie eine Karriere als Musiker.

$x, y$ und $z$ stehen für die Optionen. Dabei lässt Raz offen, ob $z$ eine tatsächlich realisierbare oder nur eine mögliche hypothetische Option darstellt. Aus den Prämissen (1), (2) und (3) folgert Raz, dass $x$ weder besser noch schlechter als noch gleich gut wie $y$ ist. Warum folgt das gemäss Raz? In der Prämisse (1) ist festgehalten, dass $x$ weder schlechter noch besser als y ist. Aus den Prämissen (2) und (3) wird gefolgert, dass $x$ und $y$ nicht gleich gut sind. Wenn zwei Optionen inkommensurabel sind, fällen wir gemäss Raz dementsprechend keine transitiven Urteile. Wenn $z$ besser als $x$ und $y$ nicht besser als $x$ ist, sollte $z$ eigentlich (wenn Transitivität gälte) auch besser als $y$ sein. Dies jedoch ist nicht der Fall: $z$ ist nicht besser als $y$. Ergo kann ein harter Fall durch das Versagen von Transitivität charakterisiert werden.

Gemäss der zweiten Strategie bildet man ein Argument mit der folgenden Form:

1. $x$ ist weder besser noch schlechter als $y$.

2. $x+$ ist besser als $x$.

3. $x+$ ist nicht besser als $y$.

$\therefore x$ ist weder besser noch schlechter als noch gleich gut wie $y$.

Ein Beispiel:

1. Eine Karriere als Anwalt ist weder besser noch schlechter als eine Karriere als Musiker.

2. Eine Karriere als Anwalt plus zusätzliche Ferien ist besser als eine Karriere als Anwalt.

3. Eine Karriere als Anwalt plus zusätzliche Ferien ist nicht besser als eine Karriere als Musiker.

$\therefore \quad$ Eine Karriere als Anwalt ist weder besser noch schlechter als noch gleich gut wie eine Karriere als Musiker. 
$x, y$ und $x+$ stehen für die Optionen. $x+$ ist eine marginale Verbesserung von $x$. Aus den Prämissen (1), und (3) folgert Raz, dass $x$ weder besser noch schlechter als noch gleich gut wie $y$ ist. Warum folgt dies gemäss Raz? In der Prämisse (1) ist festgehalten, dass $x$ weder besser noch schlechter als $y$ ist. Aus den Prämissen (2) und (3) wird geschlossen, dass $x$ und $y$ nicht gleich gut sind. Auch in diesem Fall gilt gemäss Raz, dass wir keine transitiven Urteile fällen. Wenn $x+$ besser als $x$ und $y$ nicht besser als $x$ ist, sollte $x+$ eigentlich (wenn Transitivität gälte) auch besser als y sein. Dies jedoch ist nicht der Fall: $x+$ ist nicht besser als $y$.

Die beiden Strategien sind beinahe identisch. Beide sollen gemäss Raz zeigen, dass das Versagen von Transitivität das entscheidende Merkmal von Unvergleichbarkeit darstellt. Der einzige Unterschied besteht darin, dass einmal eine dritte Option $z$ und einmal eine verbesserte Option $x+$ verwendet wird. In der Literatur wird - unter dem Namen Argument der kleinen Verbesserung - vorwiegend die zweite Strategie verwendet (vgl. etwa Chang 2002a: 667). ${ }^{2}$

Hinsichtlich des positiven Argumentationsziels dieses Kapitels - Unvergleichbarkeit zeichnet sich durch eine spezifische Form der Intransitivität und Indifferenz durch eine spezifische Form der Transitivität aus - scheint es keine Rolle zu spielen, ob eine verbesserte Option $(x+)$ oder eine dritte Option $(z)$ verwendet wird. Damit ist gemeint, dass Transitivitätsbedingungen dieselben bleiben, wenn eine bestimmte Option, zum Beispiel $x+$, stets durch eine andere Option, zum Beispiel $z$, ersetzt wird. Und auch inhaltlich scheint es keinen Unterschied zu machen: Bei jeder Person sind sowohl Fälle denkbar, in denen sie eine verbesserte Option gegenüber der ursprünglichen, nicht aber gegenüber einer dritten Option bevorzugt (das Beispiel mit der verbesserten Anwaltskarriere), als auch Fälle, in denen sie eine Option gegenüber einer zweiten, nicht aber gegenüber einer dritten Option präferiert (das Beispiel mit der Karriere als 1988, Griffin 1997, Gert 2004, Hsieh 2005, Rabinowicz 2008, Klocksiem 2010, Suzuki 2011 und Andreou 2015. Die präziseste Darstellung und Diskussion jedoch findet sich in Espinoza 2008, Rabinowicz 2009, Gustafsson und Espinoza 2010, Carlson 2011 und Gustafsson 2013. 
Feuerwehrmann). Wenn nun in einem ersten Schritt - wie dies im Argument der kleinen Verbesserung geschieht - vorausgesetzt wird, dass zwei Optionen weder besser noch schlechter als die jeweilig andere Option sind, kann in einem zweiten Schritt irgendeine der beiden Strategien gewählt und gezeigt werden, dass dabei eine gewisse Form der Transitivität versagt oder zum Teil eine bestimmte Form der Transitivität vorausgesetzt wird. Unabhängig davon, welche Variante man wählt, ist es dasselbe Versagen und dieselbe Instanziierung von Transitivität. Was dies bedeutet, kann an dieser Stelle noch nicht nachvollzogen werden. Um die Bedeutung der (In)Transitivität im Argument zu verstehen, wird dieses im nächsten Abschnitt formalisiert. Der einzige Zweck der Formalisierung besteht darin, die komplexe Rolle der (In)Transitivität deutlich zu machen. Damit wird die Grundlage dafür geschaffen, was daraus bezüglich Unvergleichbarkeit gefolgert werden kann beziehungsweise wird dieser Frage dann im letzten Teil des Kapitels nachgegangen.

\subsection{Die Rolle der (In)Transitivität im Argument der kleinen Verbesserung}

Eine Formalisierung muss adäquat sein. Dies bedeutet unter anderem, dass das nicht-formale und das formale Argument dasselbe sein muss. In einem ersten Schritt ist es deshalb sinnvoll, jede Prämisse und Konklusion des Arguments der kleinen Verbesserung, so wie es bei Raz formuliert ist, einzeln aufzuschreiben:

1. Eine Karriere als Anwalt ist nicht besser als eine Karriere als Musiker.

2. Eine Karriere als Anwalt ist nicht schlechter als eine Karriere als Musiker.

3. Eine Karriere als Anwalt plus zusätzliche Ferien ist nicht besser als eine Karriere als Musiker.

4. Eine Karriere als Anwalt plus zusätzliche Ferien ist besser als eine Karriere als Anwalt.

$\therefore \quad$ Eine Karriere als Anwalt ist weder besser noch schlechter als noch gleich gut wie eine Karriere als Musiker. 
In einem zweiten Schritt kann das Argument wie folgt formalisiert werden:
1. $\neg(x>y)$
Prämisse
2. $\neg(y>x)$
Prämisse
3. $\neg(x+>y)$
Prämisse
4. $x+>x$
Prämisse
$\mathrm{K}[\therefore] \neg(x>y) \wedge \neg(y>x) \wedge \neg(x=y)$

$x, y$ und $x+$ sind keine Variablen, sondern stellen die Optionen dar, zwischen denen der Akteur entscheidet. '>' meint 'besser als.' '<' meint 'schlechter als'. '=' meint 'gleich gut wie' (damit ist die Relation der Wertgleichheit und nicht diejenige der Identität gemeint). ' $\neg$ ' steht für eine Negation. ' $\neg(x>y)$ ' beispielsweise bedeutet, dass es nicht wahr ist, dass $x$ besser als $y$ ist.

Im formalisierten Argument - es stellt wohlgemerkt noch keinen gültigen Schluss dar - wird ersichtlich, dass das Argument der kleinen Verbesserung folgende Transitivität verletzt:

$$
(x+>x \wedge \neg(y>x)) \rightarrow x+>y
$$

Falls diese Art der Transitivität nicht verletzt wäre, könnten nicht alle vier Prämissen des Arguments der kleinen Verbesserung zusammen wahr sein, was bedeutet, dass das Argument nicht kohärent formuliert werden könnte. Gemäss dem Argument der kleinen Verbesserung ist das Antezedenz des Konditionals wahr und das Konsequenz des Konditionals falsch. Ergo ist obiges Konditional falsch. Die Bedingung ist verletzt. Es ist diese sehr spezifische Intransitivität, an welche Raz denkt, wenn er festhält, dass das Argument der kleinen Verbesserung zeigt, dass fehlende Transitivität charakteristisch für Unvergleichbarkeit ist.

Es ist wichtig zu erwähnen, dass die Philosophinnen und Philosophen von Intransitivität sprechen. Ich folge der Terminologie der philosophischen Literatur. Es geht um die Transitivität der 'besser als'-Relation, welche verletzt wird. ${ }^{3}$ In formalen Modellen, zum 
Beispiel mikroökonomischen Theorien, wird dies manchmal als negative Transitivität bezeichnet. ${ }^{4}$ Wir können die Bedingung auch wie folgt aufschreiben beziehungsweise ist die folgende Formulierung logisch äquivalent (die Wahrheitsbedingungen sind identisch):

$$
\neg(x+>y) \wedge \neg(y>x)) \rightarrow \neg(x+>x)
$$

Man kann sich dies so vorstellen, dass man vier beliebige Aussagen macht und diese Aussagen einem Logiker zeigt. Der Logiker kann nicht sagen, ob die einzelnen Aussagen wahr oder falsch sind. Dafür ist die Logik nicht zu gebrauchen. Aber er kann beurteilen, ob die vier Prämissen einen Widerspruch beinhalten (zusammen wahr oder falsch sein können). Wenn man dem Logiker die vier Annahmen des Arguments der kleinen Verbesserung zeigt, wird er sagen, dass negative Transitivität verletzt sein muss. Ansonsten würden die vier Prämissen einen Widerspruch beinhalten.

Die Rolle der (In)Transitivität im Argument ist jedoch komplizierter. Gemäss bestimmten Formalisierungen setzt das Argument der kleinen Verbesserung eine andere Art der Transitivität voraus. Diese Transitivität kommt dann ins Spiel, wenn man das Argument gültig machen und von Prämisse 3 und 4 zur Konklusion gelangen will, dass $x$ und $y$ nicht gleich gut sind (ein wesentlicher Bestandteil der Schlussfolgerung). Ich stelle im Folgenden eine Formalisierung des Arguments der kleinen Verbesserung dar, welche Transitivität als zusätzliche Annahme oder Schlussregel voraussetzt.

$\begin{array}{lll} & \text { Formalisierung, die } & \text { Transitivität involviert } \\ 3 & \neg(x+>y) & \text { Prämisse } \\ 4 & x+>x & \text { Prämisse } \\ 5 & x+>x \wedge x=y \rightarrow x+>y & \text { Transitivität } \\ 6 & \neg(x+>x \wedge x=y) & 3,5 \mathrm{MTT}^{5} \\ \mathrm{~K}[\therefore] \neg(x=y) & 4,6 \mathrm{MPT}\end{array}$

4 Breyer beispielsweise diskutiert negative Transitivität in der Mikroökonomie (Breyer 2007: 166).

5 Diese Prämisse ist im Grunde genommen bereits logisch äquivalent mit der Konklusion, weil ' $\neg(P \wedge R)$ ' und ' $\neg P \vee \neg R$ ' logisch äquivalent sind. 
Die Formalisierung zeigt, dass folgende Art der Transitivität angenommen werden muss, damit das Argument der kleinen Verbesserung gültig wird.

$$
(x+>x \wedge x=y) \rightarrow x+>y
$$

Espinoza beispielsweise nennt dieses Prinzip der Transitivität in Anlehnung an Savage indifference principle (Espinoza 2008: 130). ${ }^{6}$ Meistens wird dieses Prinzip wie folgt verstanden: Wenn zwei Optionen gleich gut sind und eine der beiden Optionen marginal verbessert wird, dann soll man die verbesserte Option bevorzugen. In diesem Zusammenhang kann es so verstanden werden, dass, wenn zwei Optionen gleich gut sind und eine der beiden Optionen marginal verbessert wird, man dann die verbesserte Option bevorzugt (ich komme im dritten Teil darauf zurück).

Dieses Prinzip stellt eine Transitivitätsbedingung dar. Würde man anstatt einer verbesserten Option $x+$ eine dritte Option $z$ wählen, würde die vorausgesetzte Transitivität folgendermassen aussehen:

$$
(x>y \wedge y=z) \rightarrow x>z .
$$

In der philosophischen Entscheidungstheorie, welche auf vier Axiomen der Transitivität basiert, stellt obige Bedingung eines dieser vier Axiome dar (Resnik 1990: 23). Gustafsson und Espinoza nennen diese Art der Transitivität 'PI-transitivity' (Gustafsson und Espinoza 2010: 755).

Gustafsson und Espinoza behaupten deshalb, das Argument der kleinen Verbesserung werde mithilfe von Transitivität generiert. Meiner Meinung nach ist diese Analyse jedoch falsch. Es gibt alternative logische Formalisierungen des Arguments der kleinen Verbesserung, die keine Transitivität voraussetzen. 
Formalisierung ohne Transitivität
$3 \neg(x+>y)$
Prämisse
$4 x+>x$
Prämisse
$5 x=y$
Prämisse
$6 \neg(x+>x)$
3,5
$7 \quad x+>x \wedge \neg(x+>x)$
$4,6 \wedge I$
$\mathrm{K}[\therefore] \neg(x=y)$
5,7 Reductio

In obiger Formalisierung wird die Annahme, dass $x$ und $y$ gleich gut sind, wie folgt ad absurdum geführt: Wir benutzen das Identitätsprädikat $(x=y)$ und substituieren in Prämisse $3 y$ und $x$. Damit erhalten wir Prämisse 6, die zusammen mit Prämisse 4 einen Widerspruch beinhaltet. Daraus kann gefolgert werden, dass $x$ und $y$ nicht gleich gut sind. Man kann den Reductio-Beweis auch wie folgt erbringen:

$\begin{array}{ll}3 \neg(x+>y) & \text { Prämisse } \\ 4 x+>x & \text { Prämisse } \\ 5 x=y & \text { Prämisse } \\ 6 x+>y & 4,5 \\ 7 x+>y \wedge \neg(x+>y) & 3,6 \wedge I \\ \text { K }[\therefore] \neg(x=y) & 5,7 \text { Reductio }\end{array}$

Halten wir fest: Die Aussage, das Argument der kleinen Verbesserung zeige, dass Unvergleichbarkeit durch ein Fehlen von Transitivität charakterisiert werden kann, ist zu allgemein. Es gibt logische Formalisierungen des Arguments, die Transitivität involvieren. Die Aussage, das Argument der kleinen Verbesserung setze notwendig Transitivität voraus, scheint falsch. Es scheint logische Formalisierungen zu geben, die keine Transitivität involvieren. Gemäss einer Formalisierung beinhaltet das Argument folgende Transitivität:

$$
(x+>x \wedge x=y) \rightarrow x+>y
$$

Auf der anderen Seite involviert das Argument der kleinen Verbesserung folgende Form der Intransitivität:

$$
(x+>x \wedge \neg(y>x)) \nrightarrow x+>y
$$


Die Intransitivität ist erforderlich, damit die Prämissen des Arguments zusammengenommen wahr sein können. Sie ist ein notwendiger Bestandteil des Arguments. Die Transitivität ist gemäss einer bestimmten Formalisierung nötig, um im Argument von den Prämissen zur Konklusion zu gelangen. Sie scheint kein notwendiger Bestandteil des Arguments zu sein.

\subsection{Was kann hinsichtlich Unvergleichbarkeit gefolgert werden?}

Inwiefern tragen die im letzten Abschnitt gemachten Überlegungen etwas zum Verständnis harter Fälle bei? Wir können diese Fragen aufteilen:

1. Welche Rolle spielt die im Argument involvierte Transitivität? Inwiefern trägt sie zum besseren Verständnis von Unvergleichbarkeit bei?

2. Welche Rolle spielt die im Argument involvierte Intransitivität? Inwiefern trägt sie zum besseren Verständnis von Unvergleichbarkeit bei?

Beginnen wir mit der ersten Frage. Sie dient dazu, uns von der aktuellen philosophischen Diskussion abgrenzen zu können.

Die Transitivität ist nötig, um die aktuelle normative Diskussion verstehen zu können. Wenn die Prämissen und die Konklusion des Arguments der kleinen Verbesserung in der Literatur als rationale Präferenzen ${ }^{7}$ oder rationale Urteile ${ }^{8}$ verstanden werden, dann soll mit diesem Argument gezeigt werden, dass etwas der Fall sein soll, nämlich dass man gewisse Urteile fällen oder gewisse Präferenzen besitzen soll. Die erste Variante wird hierbei manchmal als preference-version und die zweite als axiological-version bezeichnet (Gustafsson 2013: 434). 
Worin besteht der Zusammenhang zwischen Normativität und Transitivität? Um das nachvollziehen zu können, muss etwas ausgeholt werden:

Es existieren zahlreiche Argumente, weshalb wir transitive Präferenzen bilden sollen. Es gibt aber auch etliche Gegenargumente. Inwiefern die verschiedenen Formen von Transitivität normativ sind, ist ein umstrittener Punkt. Der Hauptgrund, weshalb wir transitive Präferenzen besitzen sollen, ist meiner Meinung nach, dass Transitivität erlaubt, unsere Präferenzen in eine einfache Struktur und somit Optionen in eine Ordnung zu bringen. Es existieren aber auch spezifischere Argumente. Ein bekanntes und umstrittenes Argument ist das sogenannte Money-pump-Argument, welches in der Ökonomie breite Verwendung findet (vgl. z. B. Cubitt und Sugden 2001). In diesem Argument können mithilfe intransitiver Präferenzen sogenannte 'Geldpumpen' gebildet werden. Die grundlegende Idee besteht darin, dass eine Person einen kleinen Geldbetrag bezahlt, um eine bessere Option zu erhalten, und aufgrund intransitiver Präferenzen Geld verliert. Weil das ceteris paribus schlecht ist, soll man transitive Präferenzen haben. Genauer funktioniert die 'Geldpumpe' wie folgt: Nehmen wir an, dass eine Person $x$ gegenüber $y$ und $y$ gegenüber $z$ und $z$ gegenüber $x$ präferiert (also in einem bestimmten Sinne intransitiv ist). Diese Person wird für einen kleinen Geldbetrag $x$ gegenüber $z$, $z$ gegenüber $y, y$ gegenüber $x$ und $x$ nun wiederum gegenüber $z$ tauschen, ad infinitum. Das heisst, dass intransitive Präferenzen dazu führen, dass die Person am Schluss über kein Geld mehr verfügt. (Wie Hans im Glück? Nein, der passt hier schlecht ins Bild.)

Die aktuelle Diskussion setzt obiges Wissen voraus. Gustafsson und Espinoza argumentieren, dass das Argument der kleinen Verbesserung nicht zeigen kann, dass Optionen unvergleichbar sein sollen. Damit meinen sie, dass das Argument nicht zeigen kann, dass Optionen nicht besser/schlechter/gleich gut sein sollen. Und damit wiederum ist gemeint, dass nicht gezeigt werden kann, dass wir keine Präferenz haben oder nicht indifferent sein sollen. Gemäss Gustafsson und Espinoza versagt das Argument der kleinen Verbesserung deshalb, weil die Gründe, welche die Wahrheit der Prämissen stützen, in Konflikt miteinander stehen. Ergo kann die Konjunktion der Prämissen nicht wahr sein. Der entscheidende Punkt in ihrer Argu- 
mentation ist, dass ein Money-pump-Argument nur unter gewissen Bedingungen zeigt, dass Präferenzen transitiv sein sollen. Falls Optionen unvergleichbar sind, so die Philosophen, führt das Versagen der Transitivität nicht dazu, dass der Spieler Geld verliert. Daraus folgern sie, dass ein money pump keinen Grund liefert, sowohl zu glauben, dass (i) Optionen unvergleichbar sein sollen, als auch, dass (ii) die im Argument involvierte Transitivität gelten soll - dies stellt die grundlegende Idee ihres Aufsatzes dar (für eine Kritik vgl. Carlson 2011). Falls die logischen Formalisierungen im letzten Abschnitt korrekt sind, übersehen Gustafsson und Espinoza allerdings, dass das Argument nicht zwingend Transitivität voraussetzt. Ich habe argumentiert, dass Transitivität kein notwendiger Bestandteil des Arguments ist.

Auf jeden Fall ist die normative Diskussion für uns nicht relevant. Der Grund dafür ist, dass die Beschreibung des realen Phänomens interessiert. Die Transitivität liefert meiner Ansicht nach jedoch keinen Beitrag zum deskriptiven Verständnis von Unvergleichbarkeit. Sie hilft uns nicht, das reale Phänomen besser verstehen zu können. Weshalb nicht? Wie im letzten Teil des Kapitels gezeigt worden ist, kann das Argument der kleinen Verbesserung folgende Form der Transitivität beinhalten:

$$
(x+>x \wedge x=y) \rightarrow x+>y
$$

Gemäss dieser Bedingung gilt: Wenn (i) der verbesserte Beruf des Anwalts besser als der Beruf des Anwalts und (ii) der Beruf des Anwalts gleich gut wie der Beruf des Musikers ist, dann ist der verbesserte Beruf des Anwalts besser als derjenige des Musikers. Diese Art der Transitivität ist trivialerweise erfüllt, weil das Antezedens falsch und ergo das Konditional wahr ist. Das Antezedens ist falsch, weil $x$ und $y$ nicht gleich gut sind. Ein Akteur denkt im Falle von Unvergleichbarkeit nicht, dass $x$ und $y$ gleich gut sind. Dies liefert aber keinen Beitrag, um Unvergleichbarkeit besser verstehen zu können.

Halten wir fest: Uns geht es nicht um Präferenzen oder Urteile, die wir haben sollen, sondern um Präferenzen, die wir besitzen und an denen wir auch nach einer Abwägung von Gründen festhalten. Hare geht davon aus, dass ein Akteur in der im Argument der kleinen Verbesserung beschriebenen Entscheidungssituation keine 
All-things-considered-Präferenz von $x$ gegenüber $y$ oder $y$ gegenüber $x$ besitzt (vgl. Hare 2010). Ich schliesse mich dieser Deutung an. Für uns ist relevant, dass ein Akteur alles in allem nicht indifferent ist.

Damit kommen wir zur zweiten Frage: Inwiefern trägt die im Argument der kleinen Verbesserung involvierte Intransitivität zum besseren Verständnis von Unvergleichbarkeit bei? Die Antwort ist einfach: Sie liefert Evidenz dafür, dass harte Fälle keine Fälle der Indifferenz sind. Sie zeigt, dass wir in harten Fällen bestimmte intransitive und in Fällen der Indifferenz oder Gleichgültigkeit bestimmte transitive Urteile fällen. ${ }^{9}$ Inwiefern wir nicht indifferent sein sollen, ist eine andere Frage; sie interessiert an dieser Stelle nicht. Ich werde im letzten Kapitel, wenn es um die normative Frage geht, wie mit Unvergleichbarkeit umgegangen werden soll, die logische Struktur des Arguments wieder aufgreifen. In diesem Zusammenhang geht es aber nur darum, ob mit der im Argument der kleinen Verbesserung implizierten Intransitivität gezeigt werden kann, dass in harten Fällen etwas der Fall oder nicht der Fall ist. ${ }^{10}$

Damit ist aber nicht gemeint, dass Intransitivität zeigt, dass die Relation der Indifferenz semantisch vage ist. Auch der andere Teil der aktuellen Diskussion rund um das Argument der kleinen Verbesserung ist für unsere Zwecke nicht relevant. Dieser stellt einen sprachphilosophischen Disput dar. Gemäss Espinoza versagt das Argument der kleinen Verbesserung deshalb, weil Komparative wie 'gleich gut' semantisch vage sein können (Espinoza 2008). Dabei orientiert sich Espinoza an Broome, der das Argument der kleinen Verbesserung selbst nicht benützt (Broome 1997, Gustafsson 2013: 435). Das heisst, dass Espinoza von der gleichen Vagheitstheorie wie Broome ausgeht, welche es erlaubt, dass etwas wahrer oder weniger wahr als etwas anderes sein kann. Mit Broome formuliert: 'One statement P, containing a vague term, is truer than another $\mathrm{Q}$ if $\mathrm{P}$ is true in every sharpening that makes $\mathrm{Q}$ true, and also true in some sharpening that does not make Q true.' (Broome 1997: 83). Espinoza nimmt diesen Gedanken auf, entsprechend stellt Wahrheit auch nach Espinoza ein graduelles Konzept dar. Mit Espinoza ausgedrückt: Es gibt sogenannte indeterminate truthness (Espinoza 2008: 131). Basierend auf dieser Idee argumentiert Espinoza, dass das Argument der kleinen Verbesserung nicht ausschliessen kann, dass es unbestimmt ist, ob die Optionen gleich gut sind (für eine Kritik an dieser Position vgl. z. B. Espinoza 2008: 136-139 und Gustafsson 2013: 438 440). Die Anwendung des Vagheitsansatzes auf das Argument der kleinen Verbesserung ist in unserem Zusammenhang deshalb nicht relevant, weil in dieser Arbeit allgemein auf einen sprachphilosophischen Ansatz zur Erklärung von Unvergleichbarkeit verzichtet wird. Vgl. die Zusammenfassung am Ende des zweiten Kapitels.

10 Es ist nicht die Rede davon, ob wir stets transitive oder manchmal auch intransitive Einstellungen besitzen. Psychologen in den späten 1960er und frühen 1970er Jahren haben zum ersten Mal empirisch zu zeigen versucht, dass unsere Präferenzen nicht immer transitiv sind (vgl. z. B. Tversky 1969). In der Psychologie und der experimentellen Ökonomie scheint es einen Konsens zu geben, dass Experimente belegen, dass wir manchmal nicht fähig sind, transitive Präferenzen zu bilden. Tversky hält fest, dass Menschen in bestimm- 
Fälle der Indifferenz oder Gleichgültigkeit verstehe ich als Entscheidungssituationen, in denen wir deshalb indifferent zwischen den zur Wahl stehenden Optionen sind, weil sie absolut gleich gut sind. Falls wir im Supermarkt zwischen identischen Produkten wählen müssen, sind wir in diesem Sinne indifferent. Man denke etwa an 'Fasnachtsküchlein', welche auch unter anderem Namen 'Frühlingsküchlein' angeboten werden.

Weshalb kann nun die im Argument der kleinen Verbesserung implizierte Intransitivität Evidenz liefern, dass harte Fälle keine Fälle der Indifferenz sind? Betrachten wir hierzu einige der im dritten Kapitel angeführten Beispieltypen von Unvergleichbarkeit:

1. Man muss sich zwischen verschiedenen Berufen entscheiden.

2. Man muss sich entscheiden, ob man dreimal pro Woche Hockey spielen oder öfters mit Freunden Bier trinken will.

3. Man muss sich entscheiden, ob man im Restaurant das leckere und ungesunde oder das gesunde und weniger leckere Menü bestellen will.

Wenden wir im Folgenden die im Argument der kleinen Verbesserung vorausgesetzte Intransitivität auf diese Fälle an:

1. Man urteilt, dass (i) eine Karriere als Anwältin plus zusätzliche Ferien besser als eine Karriere als Anwältin ist. Man urteilt, dass (ii) eine Karriere als Musikerin nicht besser als eine Karriere als Anwältin ist. Man urteilt, dass (iii) eine Karriere als Anwältin plus zusätzliche Ferien nicht besser als eine Karriere als Musikerin ist.

ten Kontexten schwache probabilistische Transitivität verletzten. Dieses Konzept besagt, dass, wenn die Wahrscheinlichkeit $x$ gegenüber $y$ zu präferieren grösser als 0.5 ist und die Wahrscheinlichkeit $y$ gegenüber $z$ zu präferieren grösser als 0.5 ist, dann auch die Wahrscheinlichkeit $x$ gegenüber $z$ zu präferieren grösser als 0.5 ist. Gegen die Studie von Tversky wurden wohlgemerkt verschiedene Einwände vorgebracht, wie etwa, dass die Teilnehmerzahl zu gering ist (keine aussagekräftige Statistik). Der entscheidende Punkt ist, dass es bei uns um etwas anderes beziehungsweise um die Frage geht, ob Akteure im Falle von Unvergleichbarkeit negative Transitivität verletzen. Soviel ich weiss, wurde dies erstmals in den Arbeiten von Kevin Reuter und mir empirisch untersucht (Messerli und Reuter 2017 und Reuter und Messerli 2017). 
2. Man urteilt, dass (i) dreimal pro Woche Hockey-Training plus drei geschossene Tore pro Training besser ist als dreimal pro Woche Training. Man urteilt, dass (ii) dreimal pro Woche Hockey-Training nicht besser als das Biertrinken mit Freunden ist. Man urteilt, dass (iii) dreimal pro Woche Hockey-Training plus drei geschossene Tore pro Training nicht besser als das Biertrinken mit Freunden ist.

3. Man urteilt, dass (i) das noch köstlichere Menü besser als das leckere Menü ist. Man urteilt, dass (ii) das gesunde Menü nicht besser als das leckere Menü ist. Man urteilt, dass (iii) das noch köstlichere Menü nicht besser als das gesunde Menü ist.

Fällt man in den obigen drei harten Fällen obige intransitive Urteile? Ja, dies scheint plausibel zu sein. Kontrastieren wir nun obige Fälle mit einem Indifferenz-Beispiel:

1. Man muss sich zwischen zwei identischen Produkten - zwei Schokoladenjoghurts - entscheiden. Fällen wir in dieser Situation die gleichen Urteile beziehungsweise folgende intransitive Urteile?

2. Man urteilt, dass (i) Schokoladenjoghurt eins plus 50-Rappen-Bon besser als Schokoladenjoghurt eins ist. Man urteilt, dass (ii) Schokoladenjoghurt eins nicht besser als Schokoladenjoghurt zwei ist. Man urteilt, dass (iii) Schokoladenjoghurt eins plus 50-Rappen-Bon nicht besser als Schokoladenjoghurt zwei ist.

Nein, das tut man natürlich nicht: (i) ist erfüllt: 50-Rappen-Bon mehr ist ceteris paribus besser als 50-Rappen-Bon weniger. (ii) ist auch erfüllt: Die beiden Joghurts sind ja identisch. Ergo ist auch keines besser. (iii) aber ist nicht erfüllt: Man urteilt, dass das Schokoladenjoghurt eins plus 50-Rappen-Bon besser als das Schokoladenjoghurt zwei ist. Es handelt sich ja um dieselben Joghurts und 50 Rappen mehr ist besser als 50 Rappen weniger. Das heisst, dass man transitive Urteile fällt. Allgemeiner formuliert, bedeutet dies, dass wir in harten Fällen intransitive Urteile der folgenden Form fällen:

$$
(x+>x \wedge \neg(y>x)) x+>y
$$


In Fällen der Indifferenz hingegen fällen wir transitive Urteile der folgenden Form:

$$
(x+>x \wedge \neg(y>x)) \rightarrow x+>y
$$

Obige Unterscheidung zwischen Transitivität und Intransitivität kann in Anlehnung an Raz als Test verwendet werden, um harte Fälle von Fällen der Indifferenz zu unterscheiden. Man fragt eine andere Person oder sich selbst, ob man in einer Entscheidungssituation manchmal obige intransitive Präferenzen bildet. Das heisst, dass ich in diesem Fall mich selbst frage und annehme, dass es bei der Leserin gleich ist. Streng genommen stellen die Aussagen in diesem Kapitel also keine sicheren Erkenntnisse dar. Kevin Reuter und ich konnten die Hypothese allerdings empirisch bestätigen (zumindest für wichtige Fälle von Unvergleichbarkeit). Wir haben in mehreren Umfragen insgesamt ca. 1000 Menschen befragt. Die Verletzung der Transitivität ist statistisch signifikant. Unsere Resultate, die wir in verschiedenen 'Follow-up studies' bestätigen konnten, sind mit extrem hoher Wahrscheinlichkeit stabil und reproduzierbar. Die Experimente liefern die erste empirische Bestätigung, dass Unvergleichbarkeit real ist und eine Mehrzahl der Menschen dabei tatsächlich Transitivität verletzt. ${ }^{11}$

Stellt das Argument der kleinen Verbesserung beziehungsweise die im Argument implizierte Intransitivität dementsprechend nicht die entscheidende Erklärung dar, weshalb Unvergleichbarkeit auftritt? Ich denke nicht.

11 Vgl. Messerli und Reuter 2017. Das Paper zeigt, dass der philosophische Mainstream richtig liegt. Das heisst, dass wir in verschiedenen Entscheidungssituationen (i) Optionen nicht als besser/schlechter/gleicht gut bewerten können und (ii) dabei Transitivität verletzen. Regan 1997 äussert beispielsweise Skepsis am philosophischen Konsens. Er argumentiert, dass harte Fälle vielleicht nicht existieren. Genauer vertritt Regan die These der sogenannten 'strong comparability' - damit ist gemeint, dass sich Optionen stets in eine Rangordnung bringen lassen (hinsichtlich eines allgemeinen Werts). Er schreibt: '(...) I am inclined to take comparability as my working hypothesis.' (Regan 1997: 150). Unsere Daten scheinen Regans Position zu widerlegen. Die Ergebnisse in Reuter und Messerli 2017 legen jedoch den Schluss nahe, dass Intransitivität nur ein Merkmal wichtiger Fälle von Unvergleichbarkeit ist (z. B. der Berufswahl). Unwichtige Fälle von Unvergleichbarkeit, z. B. Konsumentscheidungen, können nicht durch fehlende Transitivität charakterisiert werden. Die philosophische Literatur (z. B. Raz 1986 und Chang 2002a) scheint falsch zu liegen, wenn sie Intransitivität als ein generelles Charakteristikum harter Fälle darstellt. 
Einerseits sieht sich eine Akteurin nicht deshalb mit Unvergleichbarkeit konfrontiert, weil sie hinsichtlich der zur Wahl stehenden Optionen intransitive Präferenzen bildet. Optionen sind unvergleichbar, weil wir Werte nicht gegeneinander abwägen können. Es sind nicht intransitive Präferenzen, die dazu führen, dass wir zwei Berufe nicht als besser oder schlechter als den jeweilig anderen Beruf bewerten können.

Andererseits ist es wichtig zu sehen, dass ich den Begriff der Indifferenz in einem ganz bestimmten Sinne verwende: indifferente sind absolut gleichwertige Optionen. Ich meine mit Indifferenz ununterscheidbare Alternativen. Ich behaupte nicht, dass mein Argument zum Beispiel auch für sogenannte vage Gleichheit gilt. Rauschmayer plädiert beispielsweise dafür, dass das Argument der kleinen Verbesserung auch auf vage Gleichheit angewendet werden kann (Rauschmayer 2001: 124). Mit vager Gleichheit meint er, dass die Differenz zwischen den Optionen so klein ist, dass sie für uns keinen Unterschied macht. Falls Rauschmayer richtig liegt - und dafür kann man zumindest argumentieren -, ist dementsprechend auch im Falle einer vagen Gleichheit folgende Bedingung erfüllt: (i) $A$ ist weder besser noch schlechter als $B$. (ii) $A+$ ist besser als $A$. (iii) $A+$ ist nicht besser als $B$. Ein Beispiel: (i) Ein Pepsi ist weder besser noch schlechter als ein Cola. (ii) Ein Pepsi +50 Rappen ist besser als ein Pepsi. (iii) Ein Pepsi + 50 Rappen ist nicht besser als ein Cola. Ergo bilden wir zwischen Optionen, welche vage gleich sind, dieselben intransitiven Präferenzen wie im Falle von Unvergleichbarkeit. Das heisst, dass das Argument der kleinen Verbesserung keine hinreichende Bedingung darstellt, dass Unvergleichbarkeit vorkommt (es gilt auch für andere Phänomene).

Auch wenn es also Grenzen gibt, was mit dem Argument der kleinen Verbesserung gezeigt werden kann, sollte es den potenziellen und von mir erfundenen Skeptiker, der behauptet, dass Unvergleichbarkeit dasselbe wie Indifferenz ist, umstimmen. Er müsste nun erklären können - und ich sehe nicht, wie das möglich ist -, wie es sein kann, dass Unvergleichbarkeit dasselbe wie Indifferenz ist, wenn sie hinsichtlich der Rolle der (In)Transitivität nicht identisch sind. 


\subsection{Zusammenfassung}

Im ersten Teil des Kapitels wurde das Argument der kleinen Verbesserung eingeführt.

Im zweiten Teil des Kapitels wurde sichtbar gemacht, welche Rolle (In)Transitivität in diesem Argument spielt. Mit den Formalisierungen konnte einerseits nachvollzogen werden, weshalb die Aussage, das Argument der kleinen Verbesserung zeige, dass Unvergleichbarkeit durch ein generelles Fehlen der Transitivität charakterisiert werden kann, zu wenig differenziert ist. Es gibt logische Formalisierungen des Arguments, die selbst eine bestimmte Art der Transitivität involvieren. Andererseits wurde argumentiert, dass die Aussage womöglich falsch ist, dass diese Transitivität die entscheidende Prämisse im Argument der kleinen Verbesserung darstellt. Ich habe Formalisierungen des Arguments präsentiert, die keine Transitivität zu involvieren scheinen.

Im dritten Teil des Kapitels wurde gezeigt, was die im Argument involvierte Intransitivität für das bessere Verständnis von Unvergleichbarkeit beiträgt. Im Falle von Unvergleichbarkeit bilden wir spezifische intransitive Präferenzen. Im Falle von Indifferenz hingegen bilden wir spezifische transitive Präferenzen. Das heisst, dass die im Argument der kleinen Verbesserung implizierte Intransitivität Evidenz liefert, dass harte Fälle keine Fälle der Indifferenz sind. Es wurde gezeigt, dass Unvergleichbarkeit nicht dasselbe wie Indifferenz ist.

Das Konzept der Intransitivität spielt im Zusammenhang mit Unvergleichbarkeit dabei noch eine andere Rolle. Zwischen Unvergleichbarkeit und einer Standardkonzeption von Intransitivität keine Form der Intransitivität, die in diesem Kapitel breit diskutiert worden ist - besteht eine interessante Analogie. Diese Parallele wird im nächsten Kapitel aufgegriffen, wenn es darum geht, weshalb Unvergleichbarkeit ein Problem für die Rationalität darstellt. 



\section{Kapitel 6}

Unvergleich-

barkeit

und Rationalität 
Die bisherigen Kapitel behandelten die Fragen, was Unvergleichbarkeit ist, wie harte Fälle von benachbarten Phänomenen abgegrenzt werden können und welche Argumente für Unvergleichbarkeit sprechen. In diesem Kapitel geht es darum, inwiefern harte Fälle ein Problem für die Rationalität darstellen. Das Thema besteht wohlgemerkt noch nicht darin, wie wir mit Unvergleichbarkeit umgehen können (davon handelt das letzte Kapitel). Vielmehr soll die Schwierigkeit charakterisiert werden, vor welche uns unvergleichbare Optionen bei der rationalen Entscheidungsfindung stellen.

Zuerst wird Changs Position erläutert. Sie hat sich am genausten mit dem Verhältnis von Unvergleichbarkeit und Rationalität auseinandergesetzt. Ihr Standpunkt besagt, dass Vergleichbarkeit notwendig für Rationalität ist. Gemäss dieser Ansicht kann eine Akteurin zwischen unvergleichbaren Optionen nicht rational entscheiden.

Danach frage ich, welches Bild von Rationalität dabei vorausgesetzt wird. Die These, dass Vergleichbarkeit notwendig für Rationalität ist, ist zu stark. Unvergleichbarkeit stellt für bestimmte Konzepte der Rationalität ein Problem dar und für andere nicht: Unvergleichbare Optionen sind in einem bestimmten Sinne kompatibel mit (i) einer kohärentistischen Auffassung von Rationalität, (ii) einer Maximierungsstrategie und (iii) einer Satisficing-Theorie. Einzig mit einer Optimierungstheorie ist Unvergleichbarkeit nicht zu vereinbaren (die kursiv geschriebenen Rationalitätskriterien werden später erklärt).

Als drittes diskutiere ich anhand eines Beispiels, welche Sichtweise von Rationalität relevant ist. Ich komme zum Schluss, dass eine rein instrumentelle Theorie der Rationalität das Problem unvergleichbarer Handlungsoptionen nicht erfassen kann. Und auch die anderen obig genannten Ansätze greifen zu kurz, in dem Sinne, dass es im Kontext von Unvergleichbarkeit auch um die Begründung der Wahl geht. Ich argumentiere, dass Rationalität im Kontext von Unvergleichbarkeit (i) mit einer rationalen Handlung respektive Wahl gleichgesetzt werden kann, (ii) eine rationale Wahl vom Handelnden nicht verlangt, bestimmte Ziele oder Zwecke zu verfolgen, (iii) jedoch mit Gründen respektive Begründung zusammenhängt.

Viertens geht es darum, dass eine Begründung unter bestimmten Bedingungen Vergleichbarkeit voraussetzt. Ich zeige, dass Vergleichbarkeit problematisch sein kann und nicht immer notwendig ist, 
um eine rationale Wahl treffen zu können. Einerseits ist es plausibel, manchmal eine Satisficing-Begründung anzugeben. Andererseits ist es eine offene Frage, ob Vergleichbarkeit in bestimmten Kontexten irrelevant ist (etwa im Falle von Liebe, Freundschaft oder Moral). Falls Vergleichbarkeit jedoch nötig ist, und dies scheint in harten Fällen relativ oft der Fall zu sein, besteht das Problem aus der Perspektive der Akteurin in einer 'Lücke' zwischen möglichen und gewählten Optionen. Normalerweise kann diese 'Lücke' mit einer Präferenz und der Angabe von Gründen oder Werten geschlossen werden. Im Falle von Unvergleichbarkeit ist dies nicht möglich. Die Handlungsoptionen lassen sich nicht anhand einer Wertung in eine ordinale Reihenfolge bringen. In der Konsequenz gelange ich demnach in gewisser Hinsicht zum selben Ergebnis wie Chang: Es braucht Vergleichbarkeit, um eine rationale Entscheidung treffen zu können, beziehungsweise ist es gerechtfertigt zu sagen, dass die rationale Auflösung harter Fälle die Vergleichbarkeit der Optionen voraussetzt. Allerdings gilt das nicht generell, sondern nur unter bestimmten Bedingungen. Zudem setzt es ein sehr spezifisches Verständnis von Rationalität voraus.

\subsection{Diskussion von Changs Position: Vergleichbarkeit ist notwendig für Rationalität}

In der Unvergleichbarkeits- und Inkommensurabilitätsdebatte findet man beinahe keine Argumente für die These, dass die Vergleichbarkeit von Optionen eine notwendige Bedingung einer rationalen Wahl darstellt. Oftmals scheint dies implizit vorausgesetzt. Und falls explizit erwähnt, stösst man auf Aussagen folgender Art: 'Many philosophers have thought, rightly in my view, that the possibility of justified choice between two alternatives depends on their being comparable; if the alternatives cannot be compared, practical reason fails to determine at least one of the alternatives as justified.' (Suzuki 2011: 15). Oder: 'The dissertation starts from the premise that the feasibility of a rationally justified choice depends on the possibility to rationally compare the relevant options.' (Boot 2007: 7). Aber stimmt das wirklich? Stellt die Vergleichbarkeit der Optionen tatsächlich eine notwendige Bedingung einer rationalen Wahl dar? Wie wichtig 
ist Vergleichbarkeit für Rationalität? Die Schwierigkeit liegt darin, dass dies davon abhängt, welche Theorie der praktischen Rationalität gewählt wird. Grundsätzlich können nämlich zwei Auffassungen bezüglich des Verhältnisses von Vergleichbarkeit und Rationalität unterschieden werden: (1) Die Rationalität einer Entscheidung ist abhängig von der Vergleichbarkeit der Optionen. (2) Die Rationalität einer Entscheidung ist unabhängig von der Vergleichbarkeit der Optionen.

In diesem Teil geht es darum, was gemäss Chang dafür spricht, dass Vergleichbarkeit notwendig für Rationalität ist. ${ }^{1}$ Sie schreibt: 'A comparison of the alternatives is necessary to the justification of choice.' (Chang 1997: 13). Chang setzt dabei eine gerechtfertigte mit einer rationalen Wahl gleich. ${ }^{2}$

Ihre Erklärung dafür lautet wie folgt: Jeder Grund für einen Entscheid hat seine 'rechtfertigende Kraft' hinsichtlich des Vergleichs zwischen den Optionen (Chang 1997: 12). Um diese These zu stützen, erwähnt Chang das Beispiel eines Jurymitglieds eines Klavierwettbewerbs, das entscheiden muss, ob Beatrice oder Anastice besser gespielt hat, und sich für Anastice entscheidet. Wenn nun Beatrice wissen will, warum sie verloren hat, reicht es gemäss Chang nicht aus, wenn das Jurymitglied beispielsweise allein auf Anastices musikalisches Talent hinweist. In diesem Fall, so Chang, braucht es einen Vergleich, um die Entscheidung zu rechtfertigen (Chang 1997: 8). Dies kann so verstanden werden, dass der Grund, warum Anastice schön gespielt hat, keine solche 'rechtfertigende Kraft' besitzt. Beatrice hat auch schön gespielt. Dementsprechend kann die Entscheidung auf diese Weise nicht begründet werden.

Im Zusammenhang mit einem Wettbewerb ist Changs Argumentation stichhaltig. In einem Wettbewerb versuchen die Teilnehmenden in der Regel, besser zu sein als die anderen. Die Frage 'Habt ihr das Fussballspiel gewonnen?' wird mit der Erwiderung 'Die gegnerische Mannschaft hat zwei Tore geschossen' nicht beantwortet. Mehr Tore als das gegnerische Team zu erzielen, darum geht es. Analog wird Beatrices Frage nicht beantwortet, wenn man sagt, dass Anastice schön gespielt hat. Im Kontext vieler Wettbewerbe muss

1 Vgl. Chang 1997: 7-13, Chang 2013a: 18 und Chang 2015a.

2 Viele Philosophinnen und Philosophen sehen das allerdings anders (vgl. den zweiten Teil des Kapitels). 
Vergleichbarkeit vorausgesetzt werden, weil es für den Verlierer nachvollziehbar sein sollte, weshalb er unterlegen ist. ${ }^{3}$ Bei einem fairen Wettbewerb sollte ein Vergleich mit dem Gewinner möglich sein, was bedingt, dass die Standards beziehungsweise die Regeln des Wettbewerbs transparent sind. Im Fussball ist dies beispielsweise erfüllt. Das Kriterium ist die Anzahl der geschossenen Tore. ${ }^{4}$

Nun sind Wettbewerbe ohne Zweifel ein Bestandteil unseres Lebens, sei das im Sport, in der Kultur oder im Beruf. Die Frage ist jedoch, ob eine rationale Wahl stets, also in jeglichem Kontext, die Vergleichbarkeit von Optionen voraussetzt. Man kann auch sagen, dass es nicht zulässig ist, von der Charakterisierung eines Wettbewerbs ohne Argumentation allgemeinere Aussagen über das Verhältnis von Vergleichbarkeit und Rationalität abzuleiten (so wie das meines Erachtens in Chang 1997 geschieht).

Eine generellere Auseinandersetzung finden wir in Changs Aufsatz ‘Comparativism: The Grounds of Rational Choice’ (Chang 2015a). Sie vertritt darin die Ansicht, dass ausschliesslich sogenannte komparative Fakten eine Entscheidung rational machen. ${ }^{5}$ Unter einem komparativen Fakt versteht Chang dabei eine positive Relation: 'By a comparative fact, I mean a positive comparative fact.' (Chang 2015a: 5). Gemeint sind Termini wie 'besser als' oder 'gleich gut wie'.

Changs Sichtweise kann anhand des folgenden bekannten Beispiels veranschaulicht werden: Ein Akteur, dem sowohl Genuss als auch Gesundheit wichtig sind und der im Speiselokal zwischen zwei Menüs entscheiden muss (das eine ist gesund und das andere lecker), kann nur dann eine rationale Wahl treffen, wenn zwischen den Optionen ein positiver komparativer Fakt besteht. Damit kann gemeint sein,

Ich will nicht behaupten, dass dies immer gilt, z. B. auch für literarische Wettbewerbe. Inwiefern in jeglichem Wettbewerb Vergleichbarkeit hergestellt werden kann, soll an dieser Stelle nicht diskutiert werden, ist aber fraglich. Man denke beispielsweise an die Vergleichbarkeit kultureller Leistungen. Bei der Vergabe öffentlicher Subventionen (Wettbewerb um Geld) stellt dies ein Problem dar. Inwiefern lässt sich beispielsweise die kulturelle Leistung eines Museums mit der kulturellen Leistung eines Theaters oder der kulturellen Leistung einer literarischen Gesellschaft vergleichen?

Chang schreibt: 'According to comparativism, comparative facts are what make a choice objectively correct; they are that in virtue of which a choice is objectively rational or what one has most or sufficient normative reason to do.' (Chang 2015a: 1). Die zitierten Seitenzahlen entsprechen dem Text, welcher auf Changs persönlicher Homepage zu finden ist (www.ruthchang.net/selected-publications/). 
dass das eine Menü hinsichtlich Genuss und Gesundheit besser als das andere ist. Dies wiederum kann bedeuten, dass der 'stärkere' Grund darin besteht, der Gesundheit Sorge zu tragen. Oder anders formuliert, können die unterschiedlichen Werte (Genuss versus Gesundheit) alles in allem gegeneinander abgewogen werden.

Die Dialektik von Changs Argumentation besteht darin, dass sie eine indirekte Begründung nennt, weshalb Vergleichbarkeit notwendig für Rationalität ist. Dies geschieht dadurch, dass sie drei Ansätze kritisiert, welche vom Gegenteil ausgehen. Ich werde im nächsten Teil einige dieser Ideen aufgreifen, wenn es darum geht, mit welchen Rationalitätskonzepten Unvergleichbarkeit (in)kompatibel ist.

\subsection{Unvergleichbarkeit und Rationalität: verschiedene Ansätze}

Je nachdem, was unter Rationalität verstanden wird, kann eine Akteurin zwischen unvergleichbaren Optionen eine rationale Entscheidung treffen.

Erstens geht es darum, weshalb Unvergleichbarkeit und eine bestimmte Auffassung von Rationalität nicht unbedingt im Widerspruch zueinander stehen.

Zweitens wird gezeigt, dass Unvergleichbarkeit in einem bestimmte Sinne auch kompatibel mit Satisficing ist.

Drittens werde ich kurz darlegen, weshalb sich Unvergleichbarkeit und Maximierung nicht ausschliessen und zusammen mit der $\mathrm{Op}$ timierungstheorie von Rationalität denjenigen Ansatz darstellen, der nicht mit unvergleichbaren Optionen zu vereinbaren ist.

\section{Unvergleichbarkeit und kohärentistische Rationalität}

Was besagt das Konzept der kohärentistischen Rationalität und weshalb liegt in einem bestimmten Sinne kein Konflikt mit Unvergleichbarkeit vor?6

6 Überlegungen zur Kohärenz können eine Rolle spielen, wie wir mit unvergleichbaren Optionen umgehen sollen. Dafür werde ich im letzten Kapitel argumentieren. Darum geht es an dieser Stelle aber noch nicht. 
Bei diesem Ansatz geht es um Anforderungen bezüglich der Kombination von Einstellungen. Ein Akteur ist genau dann irrational, wenn er Einstellungen hat, deren Gegenstand inkompatibel sind.

Damit kann gemeint sein, dass man beabsichtigt, $A$ (irgendeinen Zweck) auszuführen, und überzeugt ist, dass $B$ zu tun notwendig ist, um $A$ auszuführen, aber $B$ zu tun nicht beabsichtigt. ${ }^{7}$ Oder es kann bedeuten, dass man sowohl einen Beruf erlernen als auch studieren will, im Wissen darum, dass nicht beides möglich ist. Und auch wenn man (i) beabsichtigt, sich mit existenziellen Fragen auseinanderzusetzen, (ii) überzeugt ist, dass sich Philosophie als einzige Disziplin mit existenziellen Fragen auseinandersetzt, und (iii) nicht beabsichtigt, Philosophie zu studieren, ist man inkohärent.

Es ist plausibel, dass eine Person inkohärent beziehungsweise irrational ist, wenn sie ihre Ziele zum Beispiel stündlich wechselt (sogenannte diachrone Inkohären $\left.z^{8}\right)$. Vereinfachend - und wie in den obigen Beispielen veranschaulicht - ist an dieser Stelle mit Inkohärenz jedoch instrumentelle und punktuelle Irrationalität gemeint. ${ }^{9}$ Dies kann so ausgelegt werden, dass man zu einem bestimmten Zeitpunkt mehrere Dinge beabsichtigt, die nicht gleichzeitig realisiert werden können, und dies auch weiss, also überzeugt ist, dass sich die Dinge nicht zusammen verwirklichen lassen.

Die weiterführende Frage, weshalb man in diesem Sinne rational sein soll, ist hier nicht Thema. Der entscheidende Punkt besteht darin, dass Unvergleichbarkeit kompatibel mit instrumenteller Rationalität ist, in dem Sinne, dass unterschiedliche Sachlagen betroffen

7 Ich meine damit nicht Fälle, in denen dies deshalb möglich ist, weil man glaubt, dass es unmöglich ist, $B$ zu tun.

8 Die grundlegende Idee besteht bei diesem Konzept darin, dass Rationalität auch mit der Art und Weise zu tun hat, wie wir unsere Einstellungen über die Zeit hinweg beibehalten und insbesondere revidieren. Ich setze an dieser Stelle voraus, dass sich die Akteurin nicht verändert hat.

9 Ich behaupte wohlgemerkt nicht, dass Irrationalität im Sinne von Inkohärenz notwendigerweise dasselbe wie instrumentelle Irrationalität ist. Es ist denkbar, dass ein Akteur im folgenden Sinne kohärent und instrumentell irrational ist: Ein Akteur, der so schnell wie möglich zum Bahnhof gelangen will und das falsche respektive ein ineffizientes Mittel wählt, scheint nicht zwingend Kohärenz zu verletzen. Für uns sind diejenigen Fälle relevant, in welchen die Konzepte im folgenden Sinne zusammenfallen: Ein Akteur hat ein Ziel und beabsichtigt die dafür notwendigen Mittel nicht. Er ist dann inkohärent und instrumentell irrational. 
sind. Gemäss einer instrumentellen Sichtweise von Rationalität ist eine Person etwa dann rational, wenn sie die für die beabsichtigten Zwecke notwendigen Mittel, von denen sie glaubt, dass sie sie erreichen kann, ebenfalls beabsichtigt. Dies hat aber nichts mit der (Un)Vergleichbarkeit der Zwecke selbst zu tun. Wir können mit Chang auch sagen, dass instrumentelle Rationalität nicht auf komparativen Fakten basiert. Die Tatsache, dass ein Mittel hinreichend dafür ist, ein bestimmtes Ziel zu erreichen, stellt keine Relation dar. Die Wahl des Mittels ist dann sozusagen the thing to do (Chang 2015a: 15).

Unvergleichbarkeit steht notabene im Konflikt mit dem Vollständigkeitsaxiom der Entscheidungstheorie (vgl. Kapitel 4). Dazu kommt, dass ein Akteur im Falle von Unvergleichbarkeit bestimmte Axiome der Transitivität verletzt (vgl. Kapitel 5). Weil man diese Axiome als Anforderungen der Kohärenz interpretieren kann, besteht in dieser Hinsicht ein Widerspruch. Es liegt in dem Sinne kein konzeptueller Konflikt vor, als ein Akteur unter Umständen zwei Dinge nicht vergleichen kann, und dies nichts mit den Mitteln zu tun hat, wie diese Dinge erreicht werden können. Ein Akteur kann genau wissen, was zu tun ist, um Anwalt oder Ökonom zu werden, aber nicht wissen, welches der beiden Ziele oder Projekte er verfolgen will. Er kann dann die Mittel beabsichtigen, um eine der beiden (unvergleichbaren) Optionen zu verwirklichen. In diesem Fall ist er instrumentell rational, obwohl Unvergleichbarkeit vorliegt. Umgekehrt kann man auch instrumentell irrational sein, obwohl die Optionen vergleichbar sind. Das Studium der Ökonomie kann alles in allem besser als dasjenige der Rechtswissenschaft sein, und trotzdem kann man es versäumen, die dafür notwendigen Mittel zu intendieren.

\section{Unvergleichbarkeit und Satisfizieren}

Was meint eine Satisficing-Theorie und weshalb liegt auch hierbei nicht zwingend ein Konflikt vor?

Die grundlegende Idee bei diesem Ansatz besteht darin, dass es rational ist, dasjenige zu wählen, was genügend gut ist (Byron 2000: 1). Das heisst, dass es nicht darauf ankommt, die beste Alternative zu präferieren. Vielmehr kann eine Option gewählt werden, welche ausreichend gut ist. 
Dieses Rationalitätskonzept geht auf Simon zurück (Simon 1953). Gemäss Simon überfordert uns die Theorie der Nutzenmaximierung in dem Sinne, dass sie kognitive Fähigkeiten voraussetzt, die wir nicht besitzen. ${ }^{10}$ Simons Modell basiert auf einer Funktion, welche zwischen den Werten 1 und 0 unterscheidet. 1 meint befriedigend (satisfactory) und 0 unbefriedigend (unsatisfactory). Der entscheidende Punkt besteht darin, dass ein Akteur jede befriedigende Option wählen darf. Man identifiziert die ausreichend guten Alternativen und darf dann jede dieser Optionen wählen (vgl. auch Byron 2000: 2-5). Anders als im Falle einer Nutzenmaximierung muss ein Akteur hierbei zum Beispiel nicht mit Wahrscheinlichkeiten kalkulieren können.

Ein Exempel: Ein Akteur, welcher ein Auto benötigt, will nicht das beste, sondern ein genügend gutes Fahrzeug kaufen. 'Gut genug' meint, dass das Auto hinsichtlich der relevanten Kriterien wie Komfort oder Preis zufriedenstellend ist. Sobald ein Akteur ein solches gefunden hat, fällt die entsprechende Wahl rational aus.

In diesem Zusammenhang wird manchmal eingewendet, dass 'gut genug' ein elliptischer Ausdruck für 'das Beste unter bestimmten Bedingungen' ist (Weber 2000: 78). Gemäss diesem Einwand entspricht das Satisfizieren einer Optimierung. Das heisst, dass die Wahl eines genügend guten Wagens aufgrund von Opportunitätskosten (das Weitersuchen braucht Zeit, Energie etc.) dennoch die beste Wahl darstellt. Anders formuliert, übersteigen die erwarteten Kosten einer zusätzlichen Evaluation den erwarteten Nutzen, ein möglicherweise besseres Auto zu finden. Dementsprechend optimiert man alles in allem genau dann, wenn man punktuelles Satisfizieren betreibt. Gemäss dieser Deutung hat Satisficing rein strategischen Wert.

Es lassen sich demnach zwei Arten des Satisfizierens unterscheiden: strategisches und genuines Satisfizieren (Weber 2000: 78). Strategisch meint, dass die Wahl einer genügend guten Option aufgrund der Kosten einer zusätzlichen Evaluation de facto die beste Option darstellt und deshalb rational ist. Genuin bedeutet, dass es rational ist, eine genügend gute Option zu wählen, unabhängig davon, ob die Kosten einer zusätzlichen Suche den potenziellen Nutzengewinn

10 Siehe Kapitel 3 zur Frage, was es konkret heisst, das Konzept der Nutzenmaximierung anzuwenden, und welche Fähigkeiten der Wahrscheinlichkeitsrechnung dabei vorausgesetzt werden. 
überwiegen. Beim genuinen Satisfizieren wählt ein Akteur eine Option, welche ein fixes Kriterium erfüllt, und weiss möglicherweise, dass bessere Optionen existieren.

Ich denke, dass Weber damit richtig liegt, dass sich nicht anhand von konkreten Beispielen entscheiden lässt, ob genuines Satisfizieren plausibel ist oder nicht (Weber 2000: 79). Je nachdem, welche Rationalitätskriterien vertreten werden, fällt die Deutung anders aus. Zu jeder Satisficing-Interpretation eines konkreten Exempels existiert eine entsprechende Optimierungs-Interpretation. ${ }^{11}$

Das bedeutet wohlgemerkt nicht, dass keine Argumente für genuines Satisfizieren existieren. Neben dem Aspekt der Überforderung wird beispielsweise vertreten, dass das Satisfizieren der Tugend der Moderatheit entspricht. Während Maximierung und Optimierung eher Gier zum Ausdruck bringen, steht Satisfizieren für Bescheidenheit (Byron 2000: 6). Ich teile allerdings die Meinung von Schmidtz, dass keine konzeptuelle Verbindung zwischen dieser Rationalitätstheorie und Bescheidenheit besteht (Schmidtz 2000). Eine ausreichend gute Wahl muss nicht moderat sein. Die Wahl eines Luxuswagens kann genügend gut und unbescheiden sein. Ein überzeugenderes Argument besteht darin, dass es manchmal schlicht nicht wichtig ist, die beste Option zu wählen: 'What seems important from a narrow perspective is simply of no significance from a larger or broader perspective.' (Weber 2000: 89). Weber meint damit, dass es rational erlaubt ist, den alltäglichen Dingen manchmal nicht zu grosse Relevanz beizumessen (Weber 2000: 91). Dies kann so verstanden werden, dass es nicht wichtig ist, das beste Fahrzeug zu kaufen. Schliesslich kann ein Auto beispielsweise lediglich ein Mittel darstellen, um zur Arbeit zu fahren.

Worin besteht nun der Zusammenhang mit Unvergleichbarkeit? Eine unvergleichbare Alternative kann genügend gut sein. Ein Auto kann genügend gut und unvergleichbar mit einem anderen Fahrzeug sein. Dementsprechend kann ein Akteur auch zwischen unvergleichbaren Optionen eine rationale Entscheidung treffen. Ergo besteht

11 In einem bestimmten Sinne ist es möglich, das Satisfizieren auf eine Optimierung oder Maximierung zu reduzieren. Man kann beispielsweise eine Präferenzrelation unterstellen, welche nur 'gute' von 'schlechten' Optionen unterscheidet. Wenn die Maximierung durch eine solche Präferenzrelation bestimmt wird, dann ist sie identisch mit Satisfizieren (Rott 2001: 148). 
kein Konflikt. Mein Punkt ist, dass Unvergleichbarkeit deshalb kein Problem für einen Satisficing-Ansatz von Rationalität darstellt, weil Vergleichbarkeit in diesem Modell keine Rolle spielt. Beim Satisfizieren ist im Gegensatz zu einer Maximierungs- und Optimierungsstrategie keine Vergleichbarkeit miteinbezogen.

Dagegen kann eingewendet werden, dass auch genügend gut auf einem Vergleich basiert. Wenn ein Auto genügend gut ist, ist dies gemäss Chang nämlich gleichbedeutend damit, dass es hinsichtlich der relevanten Kriterien wie Preis und Komfort genügend gut ist. Dies wiederum setzt nach Chang voraus, dass es mit den anderen Fahrzeugen vergleichbar ist (Chang 2015a: 20). Dieser Kritikpunkt trifft aber nicht zu. Das Charakteristikum des genuinen Satisfizierens besteht genau darin, dass es egal ist, wie komfortabel oder preisgünstig die anderen Wagen sind. Das gewählte Auto ist gut genug (es erfüllt die gewünschten Standards). Man ist zufrieden, so wie es ist.

Betrachten wir als nächstes nun die Ansätze der Maximierung und Optimierung, welche bereits mehrfach erwähnt, aber noch nicht erläutert worden sind.

\section{Unvergleichbarkeit, Maximierung und Optimierung}

Die Unterscheidung zwischen dem Konzept der Optimierung und demjenigen der Maximierung findet man beispielsweise bei Sen (vgl. Sen 1997).

Gemäss der Theorie der Optimierung ist eine Wahl genau dann rational, wenn die gewählte Option mindestens gleich gut wie die anderen Optionen ist. Dies stellt den einzigen Ansatz dar, der eindeutig nicht kompatibel mit Unvergleichbarkeit ist. In harten Fällen sind die Optionen nicht besser/schlechter/gleich gut.

Gemäss der Theorie der Maximierung ist eine Wahl genau dann rational, wenn die gewählte Option nicht schlechter als die anderen Optionen ist. Diese Theorie ist kompatibel mit Unvergleichbarkeit, weil die Optionen in einem harten Fall diese Eigenschaft aufweisen. Gegeben, dass man das Konzept der Maximierung als adäquate Theorie einer rationalen Entscheidung akzeptiert, stellt Unvergleichbarkeit nach Hsieh - und dies ist plausibel - dementsprechend kein Problem für die Rationalität dar (vgl. Hsieh 2007). 
Ich interpretiere obige Idee folgendermassen: Gemäss einer Maximierungstheorie stellt Unvergleichbarkeit deshalb kein Problem dar, weil es keine Rolle spielt, dass nicht bestimmbar ist, in welchem Verhältnis die Optionen zueinander stehen. Man kann dementsprechend nicht sagen, dass eine schlechtere Option gewählt wurde. Ergo ist die Wahl einer unvergleichbaren Option nicht irrational. Gemäss einer Optimierungstheorie hingegen spielt es eine Rolle, dass nicht bestimmbar ist, in welchem Verhältnis die Optionen zueinander stehen. Man kann nicht sagen, dass die gewählte Option mindestens gleich gut wie die andere unvergleichbare Option ist. Ergo ist die Wahl irrational.

Es ist wichtig zu erwähnen, dass die Konzepte der Optimierung und der Maximierung sehr ähnlich sind. Ein Akteur, der optimal wählt, maximiert in der Regel auch. Und ein Akteur, der maximal wählt, optimiert gewöhnlich auch. Ich meine, dass die beiden Theorien der Rationalität vom Akteur Folgendes verlangen: Wenn er die Wahl zwischen zwei Optionen hat, soll er die bessere Alternative wählen. Die beiden Theorien sind nur dann nicht identisch, wenn die ordinale Rangordnung zwischen den Alternativen nicht vollständig ist (Sen 2000: 486). Das heisst, dass sie im Falle von Unvergleichbarkeit nicht identisch sind. Wie oben dargestellt, ist gemäss einem Maximierungsansatz dann eine rationale Wahl möglich, während gemäss einem Optimierungsansatz keine rationale Entscheidung getroffen werden kann.

Zusammenfassend können wir festhalten, dass bestehende Ansätze der Rationalität kompatibel mit Unvergleichbarkeit sind. Im nächsten Teil geht es nun darum, weshalb diese Ansätze dem Problem nicht gerecht werden und welche Sichtweise von Rationalität relevant ist.

\subsection{Unvergleichbarkeit und Rationalität: Welche Sichtweise ist relevant?}

Ich werde im Folgenden mithilfe eines Beispiels von Unvergleichbarkeit - der Studienwahl zwischen Philosophie und Rechtswissenschaft - der Frage nachgehen, welche Sichtweise von Rationalität entscheidend ist. 
Zunächst können wir den Begriff der Rationalität insofern eingrenzen, als er mit dem Begriff einer rationalen Handlung gleichgesetzt wird. In einem harten Fall geht es letztlich darum, etwas zu tun respektive eine bestimmte Handlung auszuführen: Entweder studiert man Philosophie oder Rechtswissenschaft oder tut etwas Drittes. Das heisst, dass rationales Handeln mit rationalem Entscheidungshandeln gleichgesetzt wird (Hahn 2013:143). Alternative Formen des Handelns sind etwa spontanes Handeln oder Handeln aus Erfahrung oder Gewohnheit. Wie bei Schick wird hierbei davon ausgegangen, dass einer rationalen Handlung eine rationale Wahl vorangeht (Schick 1984: 9). Es ist meistens abwegig, bewusst irrational zu wählen und rational zu handeln. Es ist unplausibel, dass die Wahl, Philosophie zu studieren, irrational und die Handlung, Philosophie zu studieren, rational ist. ${ }^{12}$

Eine rationale Wahl muss dabei nicht gleichbedeutend damit sein, wie ein Ökonom wohl festhalten würde, dass ein Akteur genau dann rational ist, wenn er seinen eigenen Nutzen maximiert. Hausman schreibt, und ich denke, dass dies richtig ist: '(...) many economists think that is rational to prefer what will make oneself better off.' (Hausman 2012: 20). ${ }^{13}$ Die ökonomische Deutung stellt eine zu enge Auffassung von Rationalität dar. Ein Akteur muss weder diejenige Handlung ausführen, welche sein eigenes Interesse maximiert (Antwort auf die Frage, was man tun will), noch stets nach einer gegen dem besten Urteil handelt und sich dennoch rational verhält. Ein Beispiel: Man denkt, dass es das Beste ist, mit dem Bus in die Stadt zu fahren, wählt aber intuitiv, zu Fuss zu gehen, und realisiert im Nachhinein, dass man das Abonnement zu Hause vergessen hat. Die Rationalität solcher Handlungen kann damit gerechtfertigt werden, was man in der Psychologie rationale Intuition nennt. Eine rationale Intuition meint, dass in einem Bruchteil von Sekunden extrem viele Informationen aus einem Netzwerk von Erfahrungen ausgewertet werden und wir dabei Erkenntnisse, Erfahrungen, Argumente und situative Merkmale kombinieren. In der Philosophie existiert eine sehr ähnliche theoretische Diskussion darüber, ob sogenannte Akrasie - vereinfacht: Handeln entgegen dem besten, bewussten Urteil - rational sein kann. Siehe etwa Audi 1990 oder Arpaly 2000. Eine empirische Untersuchung dazu findet man in Messerli und Reuter (under review).

13 Dies stellt eine substanzielle Interpretation und keinen notwendigen Bestandteil ökonomischer Entscheidungsmodelle dar. Als Ökonomin muss man dies nicht behaupten. Eine Ökonomin muss das von ihr verwendete formale Modell nicht auf diese Weise interpretieren. Formale Entscheidungstheorien, so wie sie heute in der Ökonomie Anwendung finden, können als Modelle einer sehr spezifischen Form der Kohärenz verstanden werden. 
Maxime handeln, die einzig der Steigerung seiner Interessen dient (Antwort auf die Frage, welche allgemeineren Handlungsstrategien man wählen will), noch muss er ein völliger Egoist sein (Antwort auf die Frage, wie man leben beziehungsweise welcher Mensch man sein will). Falls die ökonomische Deutung stichhaltig wäre, würde dies bedeuten, dass ' $x$ ist die wertvollste Option' und ' $x$ maximiert nicht die eigenen Interessen' einen Widerspruch darstellte. Dies ist aber nicht der Fall. Es ist nicht einsichtig, weshalb nicht-egoistisches Handeln, nicht-egoistische Handlungsstrategien oder nicht-egoistische Lebensentwürfe per se irrational sein sollen. Im Gegenteil: Die Gleichsetzung von Rationalität und Maximierung oder Optimierung von Eigeninteresse ist gesellschaftlich betrachtet sogar sehr gefährlich. ${ }^{14}$ Allgemeiner formuliert lässt sich sagen, dass bei einer rationalen Wahl vom Handelnden nicht verlangt wird, dass er ein bestimmtes Ziel oder einen bestimmten Zweck verfolgt (zum Beispiel die Maximierung der eigenen Interessen). In Anlehnung an Tugendhat wird davon ausgegangen, dass uns 'keine von Natur aus vorgegebene Willensziele' gegeben sind (Tugendhat 1979: 228). In unserem Beispiel heisst dies, dass sowohl das Studium der Rechtswissenschaft als auch dasjenige der Philosophie eine rationale Wahl darstellen kann.

Die Rationalität der Wahl ist aber nicht unabhängig davon, welche Gründe für die unterschiedlichen Studiengänge sprechen. ${ }^{15}$

Denken wir etwa an eine Psychiaterin, die ein Gutachten über einen Straftäter erstellen muss. Etwas vom 'Schlimmsten', was der Gutachterin passieren kann, ist, dass der Straftäter entgegen dem Gutachten rückfällig wird. Dies kann dadurch verhindert werden, dass das Gutachten verhältnismässig zu hart ausgestellt wird. Was gesellschaftlich und persönlich optimal ist, klafft in diesem Fall unter Umständen auseinander - ich setze voraus, dass eine Gesellschaft auch ein Interesse daran haben muss, dass adäquat bestraft wird beziehungsweise die eigene Sicherheit vor potenziellen Straftätern nicht das einzige Ziel darstellt (ansonsten müsste für eine lebenslängliche Wegsperrung plädiert werden). Zahlreiche andere Beispiele können an dieser Stelle angefügt werden: Ein Lehrer, der oft nur egoistisch handelt, z. B. minimalen Aufwand betreibt, ist kein guter Lehrer. Ein Regierungsrat, der nur auf sich schaut, z. B. das eigene Image in den Medien höher einstuft als die Loyalität gegenüber seinen Staatsanwälten, ist kein guter Vorgesetzter. Ein Arzt, der einem Patienten wegen einer Profitüberlegung eine bestimmte Behandlung empfiehlt, ist kein guter Arzt.

15 Ich folge in diesem Punkt Tugendhat: 'Die Fähigkeit des Überlegenkönnens, also nach Gründen und Gegengründen fragen zu können, ist das, was man Rationalität nennt.' (Tugendhat 2003: 17). Etliche Philosophen in Broomscher Tradition sehen es allerdings anders, weil sie den Begriff der Rationalität nur auf das Verhältnis beziehungsweise die Kombination von Einstellungen, Überzeugungen etc. anwenden wollen. 
Eine rationale Wahl lässt sich demnach nicht darauf reduzieren, dass ein Akteur die Mittel zur Zielerreichung ebenfalls beabsichtigt. Ein rein instrumentelles Bild von Rationalität greift im Kontext von Unvergleichbarkeit zu kurz. So ist denkbar, dass es für einen Akteur rational ist, Philosophie zu studieren, weil gute Gründe für dieses Studium sprechen. Dies muss aber nicht gleichbedeutend damit sein, dass ein Studium der Philosophie ein Mittel darstellt, um etwas Drittes beziehungsweise ein anderes Ziel erreichen zu können. Eine Reduktion des Begriffs der Rationalität auf instrumentelle Rationalität verkennt verschiedene Dinge. Dazu zählt, wie im zweiten Kapitel erwähnt worden ist, dass es nicht-instrumentelles Nachdenken über Präferenzen gibt. Man kann Gründe dafür und dagegen nennen, etwas tun zu wollen. ${ }^{16}$ Ich meine aber auch, dass wir Dinge nicht immer einem anderen Ziel zuliebe tun; Philosophie zu studieren kann an sich Ziel (genug) sein. Und nicht zuletzt geht es im Falle von Unvergleichbarkeit um die Abwägung zwischen verschiedenen Zielen (Philosophie oder Rechtswissenschaft). Gemäss einem instrumentellen Verständnis macht die Rationalität aber bei der Erreichung der Ziele Halt. Wie im ersten Teil des Kapitels erwähnt, sind somit unterschiedliche Sachlagen betroffen.

Eine rationale Wahl erschöpft sich auch nicht in einem Maximierungs- oder Optimierungsansatz. Auch diesen Theorien liegt die implizite Annahme zugrunde, dass Präferenzen oder Ziele alle gleich rational sind. Die Prämisse, dass eine Konzeption von Rationalität neutral gegenüber den Zielen sein soll, leuchtet aber nicht ein - unabhängig davon, wie spezifisch die Ziele formuliert werden, damit ein bestimmter Typ Ziel à la Maximierung von Eigeninteresse gemeint ist oder dies offengelassen wird.

Vielmehr scheint es plausibel zu sein, der kantischen Tradition von Rationalität zu folgen, welche besagt, dass Rationalität mehr als blosse Konsistenz bedeuten sollte, auch wenn es falsch ist, rational mit moralisch gleichzusetzen. Es ist ausreichend, dass es für einen bestimmten Akteur irrational ist, nicht nach guten Gründen zu handeln.

Auf jeden Fall rechtfertigt man die Studienwahl durch die Angabe von Gründen. Es macht demnach Sinn, dass die Rationalität 
einer Entscheidung mit Rechtfertigung zusammenhängt. Ein Akteur wählt dann irrational, wenn er denkt, dass $x$ die besser begründete Option darstellt, aber dennoch $y$ wählt. Dies scheint deshalb der Fall zu sein, weil eine solche Wahl nicht gerechtfertigt werden kann. Eine Begründung sollte nicht die Form haben, dass $x$ die am besten begründete Option darstellt und deshalb $y$ gewählt wird. Wenn die Tante fragt: 'Warum hast du dich für Philosophie entschieden?' und man antwortet: 'Weil Jurisprudenz das bessere Studium ist', wird das die Tante verwirren. Wenn man sich selbst fragt: 'Will ich Philosophie studieren?' und dabei denkt: 'Ja, weil Rechtswissenschaft besser ist' ist einem alles egal. Anders formuliert heisst dies, dass man bei einer gerechtfertigten Wahl als Akteur via Deliberation zum Schluss kommt, dass das Studium der Philosophie bei Berücksichtigung aller relevanten Belange die am besten begründete Option ist. Dementsprechend entscheidet man sich für Philosophie. Gegeben, dass man nicht willensschwach, träge oder sonst irgendwie verhindert ist, zum Beispiel auf der Stelle von einem Blitz getroffen wird, endet diese Wahl auch im Handlungsversuch, ein Philosophiestudium zu absolvieren.

Wann aber ist eine Wahl begründet? Wann ist die Entscheidung gerechtfertigt, Philosophie zu studieren? Es liegt nahe anzunehmen, dass die Wahl begründet ist, wenn die besten Gründe für das Studium der Philosophie sprechen. Was ist gemeint? Ist die Wahl zum Beispiel begründet, wenn man der Tante sagt, dass man deshalb Philosophie studieren will, weil sich diese Disziplin mit sinnvollen Fragen auseinandersetzt? Wenn man sich diesbezüglich täuscht, die Philosophie keine sinnvollen Fragen zum Gegenstand hat, ist die Entscheidung nicht begründet. Oder mit Beisbart formuliert: 'Eine Begründung ist erstens dann nicht gelungen, wenn bereits dieser Verweis nicht stimmt. Ich kann die Ansicht, dass jemand in der Küche ist, nicht durch die falsche Behauptung, in der Küche sei Lärm, begründen.' (Beisbart 2007: 14). Analog kann das Studium der Philosophie nicht dadurch begründet werden, dass es sinnvolle Fragen zum Gegenstand hat, wenn de facto sinnlose Fragen diskutiert werden. ${ }^{17}$ dass objektive Begründungen nur auf Tatsachen beruhen und alle relevanten Fakten in Betracht ziehen, während subjektive Begründungen nur auf den Annahmen und Einschätzungen einer bestimmten Person beruhen. Dabei erwähnt er, dass wir es nie mit 
Ohne Zweifel kann die Tante diesen Grund nun anzweifeln, und es nicht als gute Begründung für ein Studium der Philosophie ansehen. Das bedeutet, dass die Tante einen dazu veranlasst, die Begründung zu überdenken. Nehmen wir an, dass keine Fluchtmöglichkeit besteht und man gewillt ist, die Diskussion fortzufahren. Entweder wiederholt man nun den bereits angeführten Grund - 'Ich interessiere mich für philosophische Fragen.' - oder nennt weitere Begründungen. Im ersten Fall macht man deutlich, dass man keine weiteren Argumente anführen kann oder will. Im zweiten Fall versucht man, auf weitere Gründe zu verweisen, weshalb philosophische Fragen von Bedeutung sind.

Die Grundlage für die Auseinandersetzung besteht darin, dass man der Tante einen oder mehrere sogenannte normative Gründe genannt hat. ${ }^{18}$ Darunter kann, abgrenzend zu motivierenden Gründen (das sind diejenigen Gründe, aus denen heraus eine Akteurin handelt), ein Faktor verstanden werden, der für eine bestimmte Handlung spricht. ${ }^{19}$

Wann jedoch kommt die Begründung an ihr Ende? Hierbei nach einem archimedischen Punkt zu suchen, wäre verfehlt (Nida-Rümelin 1999: 188). Im Alltag ist sie in der Regel abgeschlossen, wenn 'kognitiver Friede' herrscht, zum Beispiel das Essen serviert wird. Mit obigem Beispiel soll aber etwas anderes gezeigt werden beziehungsweise ist mein Punkt ein anderer, nämlich, dass die Frage nach den besten Gründen beliebig weitergeführt werden kann. Das Exempel soll aber auch darlegen, dass die Begründungskette irgendwann abbricht. Wir

objektiven Begründungen zu tun haben beziehungsweise unser Wissen über eine Handlungssituation immer unvollständig, die Unterscheidung zwischen objektiven und subjektiven Gründen jedoch nützlich ist, wenn man zwei Perspektiven kontrastieren möchte, von denen die eine besser informiert ist als die andere (Beisbart 2007: 27). Was heisst dies in unserem Fall? Einerseits gehe ich wie gesagt davon aus, dass die Überzeugung, dass die Philosophie sinnvolle Fragen zum Gegenstand hat, wahr ist. Andererseits nehme ich an, dass man den Entschluss, Philosophie zu studieren, aufgrund einer besseren faktischen Informationslage nicht revidieren würde.

18 Scanlon spricht von Gründen in einem 'standard normative sense' (Scanlon 1998: 19). Raz bezeichnet normative als leitende Gründe ('guiding reasons'), was mir ebenfalls passend scheint, da normative Gründe im Kontext einer Handlung dem Zweck der Orientierung dienen (Raz 1978: 3).

19 Motivierende Gründe liefern Handlungserklärungen, während normative Gründe eine Handlung begründen können. Dies heisst nicht, dass kein Zusammenhang besteht. Eine Handlung kann manchmal erklärt werden, indem man sie aus der Sicht der Akteurin begründet (vgl. Beisbart 2007: 25). 
besitzen rein theoretisch die Möglichkeit, sämtliche unserer Gründe in Zweifel zu ziehen: Soll ich mich mit Philosophie auseinandersetzen, einen Beruf erlernen, arbeiten, eine Familie gründen, eine Weltreise machen, anderen Menschen helfen, zum Coiffeur gehen, Bücher lesen? Einige dieser Dinge machen wir ihrer selbst willen und andere anderen Dingen zuliebe. Hinterfragen können wir beides. Im ersten Fall, bei den sogenannt intrinsisch wertvollen Dingen, können wir beispielsweise hinterfragen, ob wir tatsächlich Kinder haben, anderen Menschen helfen oder eine Weltreise machen wollen. Im zweiten Fall, bei den sogenannt instrumentell wertvollen Dingen, können wir zum Beispiel hinterfragen, ob der Coiffeur-Besuch das eigene Aussehen tatsächlich verbessert. Welche Dinge in welche Kategorie fallen, ist uns dabei wohl manchmal selbst nicht klar. Auch kann ein Mittel beziehungsweise etwas instrumentell Wertvolles mit der Zeit intrinsisch kostbar werden (oder umgekehrt), wobei wir oft gar nicht wirklich aktiv beeinflussen können, was und wie hoch wir etwas wertschätzen. Das Hinterfragen aber ist wichtig. Ein sinnvolles Leben, so könnte man sagen, reflektiert sich selbst. Mit 60 Jahren zu realisieren, dass man vielleicht doch nicht in der Anwaltskanzlei des Vaters hätte arbeiten sollen, hat etwas Tragisches. Irgendwann, und das ist der entscheidende Punkt, kommt das Hinterfragen aber an ein Ende. Irgendwann muss die Begründung zu einem Schluss kommen, wenn man etwas tun will. Man könnt einwenden, dass dies eine zu pragmatistische Betrachtungsweise darstellt, in dem Sinne, dass eine Begründung vielmehr mit dem Verweis auf unsere 'tiefsten' Bekenntnisse an ein Ende gelangt: 'Liebe und Freiheit sind das Wichtigste im Leben'; 'Ich will so viel Gutes wie möglich tun'; 'Offenheit, Haltung und Mitgefühl sind zentral'; 'Genuss ist die Hauptsache im Leben'; Ich bin ein Rechtsradikaler'. Ich will das an dieser Stelle jedoch nicht gegeneinander ausspielen. Die schwächere These, welche ich vertrete, besagt, dass wir manchmal und ab einem gewissen Punkt die Güte eines Grundes nicht weiter anzweifeln sollten, um uns nicht zu 'blockieren'. Auch dann, wenn es uns, rein theoretisch gesehen, stets möglich wäre, nach weiteren Gründen oder höheren Zwecken zu fragen. Auch dann, wenn wir uns in der Begründung vielleicht irren.

In dieser Problematik sollten wir uns jedoch nichts vormachen. Wir kommen mit unseren Argumenten relativ schnell an ein Ende, 
sei das bei der Frage, weshalb man Philosophie studieren, ein Buch lesen oder auf den Mount Everest klettern will - auch wenn das für die überrationalisierte Philosophie eine schwierige Pille zu schlucken ist. Der erste Bergsteiger, welcher den Everest bestieg, antwortete auf die Frage, weshalb er es tue, etwa wie folgt: 'Weil ich da bin.' Dies ändert aber nichts daran, dass die Wahl des Studiums unbegründet ist, wenn die Tante einen umstimmen kann oder das andere Studium besser ist - wohlgemerkt nicht durch (effektive) Dinge wie Zwang, Machtausübung oder Manipulation.

Zusammenfassend können wir festhalten, dass es im Kontext von Unvergleichbarkeit plausibel erscheint, dass eine rationale Wahl einen nicht darauf festlegt, etwas Bestimmtes zu studieren, die Wahl des Studiums jedoch mit einer Begründung zusammenhängt.

Inwiefern jedoch setzt eine Begründung die Vergleichbarkeit der Optionen voraus? Basiert eine Begründung tatsächlich auf den besten Gründen, oder ist es nicht ausreichend, genügend gute Gründe anzugeben? Wo stösst Vergleichbarkeit an Grenzen? Ist es gerechtfertigt zu sagen, dass eine Begründung Vergleichbarkeit voraussetzt? Und worin besteht nun das Problem harter Fälle? Darum geht es im letzten Teil des Kapitels.

\subsection{Vergleichbarkeit, Begründung und das Problem harter Fälle}

Der letzte Teil gliedert sich wie folgt: Erstens zeige ich bestimmte Grenzen der Vergleichbarkeit auf. Zweitens geht es darum, weshalb es dennoch gerechtfertigt ist zu sagen, dass die rationale Auflösung harter Fälle oft Vergleichbarkeit voraussetzt. Drittens versuche ich die konkrete Schwierigkeit zu charakterisieren, welche Unvergleichbarkeit bei der rationalen Entscheidungsfindung generiert.

\section{Grenzen der Vergleichbarkeit}

Vergleichbarkeit stösst in verschiedener Hinsicht an Grenzen. Einerseits kann sie negative Konsequenzen haben. Andererseits gibt es Dinge, welche unter Umständen nicht verglichen werden sollen, 
beziehungsweise setzt eine Begründung nicht immer Vergleichbarkeit voraus. Beginnen wir mit dem ersten Punkt.

An Kierkegaards Aussage, dass 'Vergleichbarkeit das Ende des Glücks und der Anfang der Unzufriedenheit darstellt' ist sicherlich etwas Wahres. Rationalität, so könnte man anführen, sollte doch weder zum einen noch zum anderen führen. Es ist deshalb wichtig zu betonen, dass es um die ganz spezifische Frage geht, ob Vergleichbarkeit im Kontext einer Entscheidung notwendig für Rationalität ist. Es ist nicht die Rede davon, darauf weist Kierkegaard hin, dass man generell nicht zu viel vergleichen soll. Abgesehen davon, dass dies in der Regel nichts bringt, kann es auch zu Unzufriedenheit führen. Damit meine ich, dass eine negative Konsequenz des Vergleichens darin besteht, dass man sich unnötig unter Stress setzt, da die anderen zum Beispiel erfolgreicher, intelligenter, schöner oder kreativer sind. Ein ständiges Vergleichen untergräbt also wichtige Dinge. So kann darunter beispielsweise nicht nur die Gelassenheit, sondern auch der Genuss leiden. Eine Person, welche sich nie mit dem Status quo zufrieden gibt respektive diesen immer vergleicht, wird ihr Leben in der Tendenz weniger geniessen können. Eine andere negative Konsequenz besteht darin, dass ein ständiges Vergleichen einen davon abhalten kann, längerfristige Ziele und Projekte zu realisieren. Wenn ein Akteur sein Studium ständig mit anderen Studiengängen vergleicht, kann dies ein Problem darstellen. Jemand, der grundsätzlich keine evaluativen Bindungen eingehen will, wird weder ein komplexeres Projekt beenden noch zum Beispiel über längere Zeit verheiratet bleiben. Das ständige Vergleichen scheint diese Art von Bindungen eher zu unterminieren. Dementsprechend wird auch die Fähigkeit beschnitten, das Studium der Philosophie über die Zeit hinweg realisieren zu können. In der Konsequenz heisst dies, dass wir weder zu viel vergleichen noch ständig neue Entscheidungssituationen suchen sollten. Auch sind Argumente denkbar, sich gegebenenfalls nicht in spezifische Entscheidungssituationen zu begeben. So existieren gute Gründe, nicht mit der Freundin eines Freundes auszugehen, wenn man Gefallen an dieser Person hat und weiss, dass dieser unter dem Einfluss von Alkohol nicht kleiner werden wird. Oder man denke an eine Person, die keine einzige Sekunde ihres Lebens damit vergeuden will zu überlegen, was sie anziehen soll, und dementsprechend nur 
identische Kleider besitzt. Das bedeutet, dass Entscheidungssituationen manchmal aus moralischen (Sex mit der Freundin des Freundes) oder aus persönlichen Gründen (nicht überlegen, was man anziehen soll) vermieden werden sollen.

Es ist denn auch eine offene Frage - das führt uns zum zweiten Punkt -, ob Optionen unter gewissen Bedingungen, nämlich wenn spezifische Dinge involviert sind, nicht verglichen werden sollen. Damit meine ich zum Beispiel Freundschaft, Liebe, Pflichten oder Verantwortung. So kann man es schlicht für verfehlt halten, die eigene Frau mit anderen Frauen zu vergleichen. Oder man denke an eine Person, welche die Verantwortung gegenüber der eigenen Tochter gegen eine Weltreise abwägt und verschwindet. Diese Person scheint nicht verstanden zu haben, dass sich die Beziehung zu einem Kind nicht auf gleiche Weise kündigen lässt wie das Abonnement im Fitnesscenter. Auf jeden Fall ist es unbestritten, dass bestimmte Dinge starke Verbindlichkeiten generieren. Ebenso scheint mir klar zu sein, dass man kein Opportunist sein sollte, der seine Überzeugungen wie ein 'Fähnchen im Wind' wechselt oder ständig nur die Konsequenzen gegeneinander abwägt und nie aus Prinzip handelt. Eigene moralische Grundsätze, sofern man welche hat, bei unangenehmen Konsequenzen über Bord zu werfen, kann nicht das Ziel sein. Und auch Loyalitäten sollten unabhängig von Vor- und Nachteilen eingehalten werden (Wolf 2005: 17). Zwischen der Überlegung, dass bestimmte Dinge grundsätzlich nicht verglichen und Entscheidungssituationen manchmal aus moralischen Gründen vermieden werden sollen, besteht denn auch ein enger Zusammenhang. Man kann sagen, dass 'eine Akteurin sollte alles in allem $x$ tun' nicht zwingend damit gleichgesetzt werden kann, dass $x$ für die Akteurin die wertvollste Option darstellt. Alternative Deutungen lauten, dass eine Akteurin $x$ tun sollte, weil dies geboten oder richtig ist. Die offene Frage ist, welche Rolle Vergleichbarkeit hierbei spielt. ${ }^{20}$ lexikographische Priorisierung im ersten Kapitel. Einige Ausführungen hinsichtlich der Frage, ob deontische Fakten tatsächlich keine sogenannte weighing explanation aufweisen, darunter kann meiner Ansicht nach eine Erklärung verstanden werden, die auf einem Vergleich basiert, finden wir auch in Broome 2013: 58-60. 
In der Konsequenz heisst dies, dass eine rationale Entscheidung unter Umständen - je nachdem, welche Rolle Vergleichbarkeit im Kontext von deontologischen Überlegungen und Idealen wie romantischer Liebe spielt - nicht durch einen Vergleich, sondern zum Beispiel durch die Loyalität und die Liebe gegenüber dem eigenen Mann oder durch die Verantwortung gegenüber dem eigenen Kind zustande kommt. Zwischen starken deontologischen Forderungen und persönlichen Idealen besteht wohlgemerkt eine grosse Differenz. Wer das Ideal romantischer Liebe nicht teilt, braucht sich auch nicht daran zu orientieren. Die Verantwortung gegenüber einem Kind hingegen scheint nicht verhandelbar zu sein. In beiden Fällen stellt es jedoch eine offene Frage dar, welche Rolle Vergleichbarkeit einnimmt.

An dieser Stelle ist es nötig, kurz innezuhalten und nochmals zu erläutern, worum es in diesem Abschnitt geht und worin der $\mathrm{Zu}-$ sammenhang zu Unvergleichbarkeit besteht. Es geht darum, dass Unvergleichbarkeit kein Problem für die Rationalität darstellt, wenn es Situationen gibt, in denen Vergleichbarkeit irrelevant ist. Mögliche Beispiele sind Moral oder Liebe. Man könnte sogar noch einen Schritt weitergehen und die kontroverse These vertreten, dass die Rationalität in solchen Kontexten fordert, nicht zu vergleichen.

Selbst wenn sich allerdings das Gegenteil herausstellen sollte, nämlich, dass Vergleichbarkeit auch in obig beschriebenen Kontexten nötig ist, um eine rationale Entscheidung treffen zu können, ist Vergleichbarkeit nicht notwendig für Rationalität.

Einerseits ist dies deshalb der Fall, weil manchmal eine Satisficing-Begründung genannt werden kann. Denken wir an das AutoBeispiel. Ich habe dafür plädiert, dass genuines Satisfizieren in solchen Fällen plausibel ist. Neben dem Aspekt der Überforderung wurde als wichtigstes Argument der Umstand genannt, dass den Dingen nicht immer eine entscheidende Bedeutung beigemessen werden muss. Falls es zutrifft, dass es manchmal ausreichend ist, aus Erfahrung oder aus guten Gründen zu handeln, und eine Option manchmal genügend gut sein kann, dann widerspiegelt sich dies auch in der Begründung. Ein Akteur kann dann den Kauf eines Fahrzeugs damit rechtfertigen, dass er es benötigt, um zur Arbeit zu fahren, und es genügend komfortabel ist. Und falls ihn jemand darauf hinweist, dass er einen besseren Wagen hätte kaufen können, kann er erwidern, dass er zu- 
frieden ist, so wie es ist. Inwiefern Tugendhat also damit richtig liegt, dass jegliche Stellungnahme in praktischer Hinsicht unweigerlich von folgender Frage begleitet wird, ist fraglich: 'Was ist gut, besser, das Beste?' (Tugendhat 1979: 194). Im Auto-Beispiel kann man aus guten Gründen $x$ tun, im Wissen, dass $y$ besser ist, und diese Wahl begründen.

Andererseits ist dies deshalb der Fall, weil unserem Handeln oftmals keine Deliberation oder Begründung vorangehen muss. Es kann Sinn ergeben, bei der Entscheidungsfindung nicht zu überlegen. In einer gefährlichen Situation kann die wertvollste Option zum Beispiel darin bestehen, so schnell wie möglich wegzurennen. Hierbei kann das Nachdenken darüber, welches die beste Option darstellt, nicht nur unnötig, sondern sogar gefährlich sein. Jeder, der einmal in eine gefährliche Situation geraten ist, weiss jedoch, dass sich diese Frage nur theoretisch stellt. In lebensbedrohlichen Situationen reagieren wir oft, ohne zu überlegen. Ohne Überlegung beziehungsweise Abwägung braucht es allerdings auch keine Vergleichbarkeit.

\section{Weshalb die Auflösung harter Fälle oft Vergleichbarkeit voraussetzt}

Auch wenn eine rationale Entscheidung folglich nicht zwingend die Vergleichbarkeit der Optionen bedingt, ist die Annahme plausibel, dass die rationale Auflösung der verschiedenen harten Fälle oft die Vergleichbarkeit der Alternativen voraussetzt.

Es ist sinnvoll, sich über die Studienwahl Gedanken zu machen. Das heisst, dass es ein Nachdenken darüber voraussetzt, welches das bessere Studium ist. Dies muss ja erst einmal erkannt werden. Die gelungene Abwägung ihrerseits, so denke ich, verlangt in diesem Fall, dass sich die relevanten Gründe und Werte vergleichen lassen. Ist dies nicht möglich, lässt sich auch nicht angeben, welches das bessere Studium ist. Die Deliberation kann in diesem Fall nicht erfolgreich abgeschlossen werden.

Harte Fälle müssen auch nicht Entscheidungssituationen darstellen, welche grundsätzlich vermieden werden sollen. Ein Akteur muss die Wahl zwischen dem Studium der Rechtswissensschaft und der Philosophie nicht unbedingt aus moralischen oder persönlichen Gründen vermeiden. 
Ebenso wenig müssen harte Fälle Entscheidungssituationen repräsentieren, welche Dinge involvieren, die grundsätzlich nicht verglichen werden sollen. Konstitutive Inkommensurabilität ist für die Studienwahl irrelevant.

Und nicht zuletzt will man in wichtigen harten Fällen nicht satisfizieren (zumindest auf den ersten Blick nicht). Die Entscheidung zwischen Philosophie und Rechtswissenschaft kann für einen Akteur eine grosse Relevanz haben. ${ }^{21}$

Betrachten wir nun als Nächstes, inwiefern harte Fälle eine Schwierigkeit bei der Entscheidungsfindung darstellen.

\section{Das Problem harter Fälle}

Harte Fälle stellen auf jeden Fall ein Problem bei der Entscheidungsfindung dar. Dies wird besonders dann klar, wenn man sich vergegenwärtigt, warum in nicht harten Fällen eine rationale Entscheidung möglich ist. Nicht harte Fälle stellen Entscheidungssituationen dar, in denen wir eine Handlungsoption bevorzugen oder indifferent zwischen den Optionen sind. In einem nicht harten Fall lässt sich die Präferenz nämlich grundsätzlich in einer Wahl ausdrücken. Eine Präferenz ermöglicht sozusagen den Übergang von den möglichen zu den gewählten Alternativen (vgl. hierzu etwa De Jonge 2012: 7). Anders formuliert, kann eine Präferenz die 'Lücke' zwischen möglichen und gewählten Alternativen schliessen. Eine Präferenz von $x$ gegenüber $y$ lässt sich in der Wahl von $x$ ausdrücken. Eine Präferenz von $y$ gegenüber $x$ lässt sich in der Wahl von $y$ ausdrücken. Wenn ein Akteur die Präferenz bildet, dass es alles in allem besser ist, sich vegetarisch als karnivor zu ernähren, dann kann er dies dadurch ausdrücken, dass er vegetarisch lebt. In harten Fällen hingegen ist es dem Akteur nicht möglich, in einer Wahl zu manifestieren, was er denkt.

In diesem Punkt gibt es eine interessante Parallele zwischen Unvergleichbarkeit und folgender Konzeption von Intransitivität:

$$
x>y>z>x
$$


Wenn ein Akteur die Wahl zwischen diesen drei Optionen hat, kann er ebenfalls durch keine Wahl angeben, welche er am wertvollsten findet. Man kann sich vorstellen, dass es besser ist, Anwalt zu sein als Ökonom, Ökonom als Journalist und Journalist als Anwalt.

Analog zu einem harten Fall kann die Handlung nicht widerspiegeln, was eine Akteurin denkt. Man könnte auch sagen, dass es in harten Fällen wie in obiger Situation keinen Übergang qua Präferenz von den realisierbaren zu den gewählten Alternativen gibt. Ich orientiere mich hierbei an einer Idee, welche der Entscheidungstheorie zugrunde liegt: ' (...) human action is described as the upshot of two selection procedures. First, from all possible (and relevant) action alternatives those alternatives are selected which are feasible. Second, from this set of alternatives the preferred one is chosen.' (De Jonge 2012: 8). Die Unvergleichbarkeit verunmöglicht das Vollziehen des zweiten Schritts. Ein Akteur kann zwar unter den relevanten die realisierbaren Optionen ausmachen, dann aber wird das Prozedere unterbrochen.

Die beiden Fälle sind notabene nicht ganz identisch: Bei obiger intransitiver Konstellation der Präferenzen besteht das Problem darin, dass eine nicht gewählte Option stets besser als die gewählte ist: Wählt der Akteur den Beruf des Anwalts, wäre der Beruf des Journalisten besser gewesen. Wählt der Akteur den Beruf des Journalisten, wäre der Beruf des Ökonomen besser gewesen. Und wählt der Akteur den Beruf des Ökonomen, wäre der Beruf des Anwalts besser gewesen. In harten Fällen hingegen besteht das Problem nicht darin, dass eine Option stets besser als die gewählte ist, sondern darin, dass im paarweisen Vergleich keine Alternative die wertvollste Option darstellt. ${ }^{22}$

Es ist also die 'Lücke' zwischen möglichen und gewählten Alternativen, welche im Falle von harten Fällen das Problem für die Rationalität generiert. Aber wie kann diese 'Lücke' genauer verstanden werden? Es sind zwei Explikationen denkbar:

Erstens kann die Handlung zwischen unvergleichbaren Optionen nicht mit einer Präferenz beziehungsweise einer Evaluation erklärt werden (aus der Dritt-Person-Perspektive). Wenn das Studium 
der Philosophie und das Studium der Jurisprudenz für einen Akteur unvergleichbar sind, kann die Handlung des Akteurs, zum Beispiel, dass er sich an der Universität für Jurisprudenz einschreibt, nicht damit erklärt werden, dass er eine Präferenz für Jurisprudenz besitzt. Diese Handlung kann also in dem Sinne nicht rational erklärt werden, als nicht allgemein verständlich und nachvollziehbar wird, warum der Handelnde in einem harten Fall auf bestimmte Weise agiert hat.

Zweitens, und diese 'Lücke' ist für die vorliegende Arbeit von Bedeutung, kann ein Akteur nicht aufgrund einer Präferenz beziehungsweise Evaluation entscheiden, was so verstanden werden kann, dass der Akteur nicht auf der Basis einer Wertung entscheiden kann (aus der Ersten-Person-Perspektive). Es gibt kein handlungsleitendes Urteil, welche Option alles in allem besser ist, und im Gegensatz zu einer Indifferenz, die streng genommen auch nicht handlungsleitend ist, ist dies nicht egal.

\subsection{Zusammenfassung}

Im ersten Teil des Kapitels wurde Changs Position eingeführt, welche besagt, dass Vergleichbarkeit notwendig für Rationalität ist. Ich habe dafür plädiert, dass es nicht zulässig ist, von der Charakterisierung von Wettbewerben allgemeinere Aussagen über das Verhältnis von Rationalität und Vergleichbarkeit abzuleiten.

Im zweiten Teil des Kapitels wurde der Frage nachgegangen, welches Bild von Rationalität dabei vorausgesetzt wird. Ich habe begründet, weshalb bestimmte Konzepte der Rationalität mit Unvergleichbarkeit kompatibel sind.

Im dritten Teil des Kapitels habe ich gezeigt, dass Rationalität im Zusammenhang mit Unvergleichbarkeit mit der Begründung der Wahl zusammenhängt.

Im vierten Teil des Kapitels wurden bestimmte Grenzen der Vergleichbarkeit dargelegt, Argumente genannt, weshalb eine Begründung dennoch oft Vergleichbarkeit voraussetzt, und erläutert, dass das Problem harter Fälle darin besteht, dass die 'Lücke' zwischen den möglichen und gewählten Alternativen nicht mit einer Präferenz beziehungsweise der Angabe von Gründen oder Werten geschlossen werden kann. 
Die daraus resultierende Konsequenz ist, dass in vielen harten Fällen keine rationale Wahl möglich scheint - weil es alternative Strategien gibt, in harten Fällen Vergleichbarkeit herzustellen. Einerseits ist es denkbar, dass harte Fälle nicht mit Unvergleichbarkeit gleichgesetzt werden dürfen, da sich Optionen durch etwas anderes als eine Wertung in eine ordinale Rangordnung bringen lassen (vgl. Kapitel 10). Andererseits ist es nicht ausgeschlossen, dass mehr als drei Vergleichsrelationen existieren. Die Optionen könnten in harten Fällen demnach aufgrund einer alternativen Vergleichsrelation als vergleichbar ausgewiesen werden (vgl. die nächsten beiden Kapiteln). 

Kapitel 7

Die TrichotomieThese und vage Entscheidungskriterien 
Das letzte Kapitel endete damit, dass (noch) nicht ausgeschlossen werden kann, dass sich in harten Fällen Vergleichbarkeit erzielen lässt. In diesem Kapitel geht es um eine mögliche Lösung, die Chang, Parfit und Griffin zur Diskussion stellen. Ich fokussiere dabei auf Chang, weil sie sich am genausten damit auseinandergesetzt hat. So wie das Problem der Unvergleichbarkeit in der Einleitung eingeführt worden ist, sind es drei Annahmen, welche zur Konklusion führen, dass in harten Fällen keine rationale Entscheidung möglich ist. Zwei dieser Prämissen - und ich stimme überein - hält Chang für richtig: Einerseits geht sie davon aus, dass die Vergleichbarkeit der Handlungsoptionen eine notwendige Bedingung einer rationalen Wahl darstellt. Im letzten Kapitel wurde gezeigt, dass Vergleichbarkeit zwar nicht notwendig für Rationalität ist, es aber plausibel ist, dass die Auflösung harter Fälle oft Vergleichbarkeit voraussetzt. Andererseits supponiert Chang, dass harte Fälle existieren. Anders formuliert sehen wir uns manchmal mit Entscheidungssituationen konfrontiert, in denen die Optionen weder besser noch schlechter noch gleich gut sind. Ich habe in früheren Kapiteln gezeigt, dass es diese Kasus gibt.

Gemäss Chang, Griffin (1998: 86) und Parfit (1986: 431) ist es jedoch falsch, dass zwei Optionen nur dann vergleichbar sind, wenn sie entweder besser oder schlechter als oder gleich gut wie die jeweilig andere Option sind. Im Falle von Chang heisst das, dass zwischen zwei Optionen eine vierte Vergleichsrelation bestehen kann, die Chang als 'Parität' bezeichnet. In harten Fällen können zwei Optionen demnach on a par respektive vergleichbar sein.

Der Schwerpunkt in Changs Forschung liegt dabei nicht in einer positiven Explikation von 'Parität', sondern in der Diskussion der sogenannten Trichotomie-These. Sie argumentiert gegen diese traditionelle Sichtweise von Vergleichbarkeit, welche besagt, dass sich Vergleichbarkeit in der dreifachen Relation von Optionen erschöpft. Damit will Chang die Möglichkeit für eine alternative Vergleichsrelation namens 'Parität' schaffen. Die Frage, wie man 'Parität' interpretieren kann, wird im nächsten Kapitel diskutiert.

Dieses Kapitel bringt die aktuelle Forschung in zweierlei Hinsicht weiter. Erstens wird das wichtigste Argument gegen die Trichotomie-These - das sogenannte 'Ketten-Argument' - erstmals 
in symbolischer Sprache rekonstruiert. Zweitens entwickle ich eine neue positive Theorie, weshalb das 'Ketten-Argument' nicht funktioniert. Diese Theorie basiert auf vagen Entscheidungskriterien.

Das Kapitel gliedert sich in sieben Teile: Erstens wird die Trichotomie-These erläutert. Zweitens wird Changs Argumentation gegen die Trichotomie-These eingeführt. Drittens wird das 'Ketten-Argument' in symbolischer Sprache rekonstruiert. Viertens erwähne ich die neusten Erkenntnisse aus der philosophischen Literatur. Ich zeige in welchem Zusammenhang dieses Kapitel zu dieser Forschung steht. Fünftens führe ich ein Gegenbeispiel gegen das Ketten-Argument ein. Sechstens betrachte ich eine mögliche Kritik. Und letztlich entwickle ich eine neue Theorie, die zeigt, dass die potenzielle Kritik nicht stichhaltig ist. ${ }^{1}$

\subsection{Die Trichotomie-These}

Der Begriff der Trichotomie kommt ursprünglich aus dem Griechischen und bedeutet eine Dreiteilung, womit in diesem Fall gemeint ist, dass drei Vergleichsrelationen existieren: 'ist besser als', 'ist schlechter als' und 'ist gleich gut wie'. Der Name Trichotomie-These geht meines Wissens auf Chang zurück. ${ }^{2}$ Nach Chang lautet diese Überzeugung folgendermassen: 'If two items, $x$ and $y$, are evaluatively comparable, then $x$ must be better or worse than $y$, or $x$ and $y$ must be equally good.' (Chang 2002a: 660).

In dieser 'Definition' stellt die Instanziierung einer klassischen Vergleichsrelation allerdings nur eine notwendige Bedingung für Vergleichbarkeit dar. Das Konditional gilt aber auch in die andere Richtung. Das heisst: Wenn $x$ entweder besser oder schlechter als oder gleich gut wie $y$ ist, dann sind $x$ und $y$ vergleichbar. Dies wird auch von Chang akzeptiert.

Die Trichotomie-These, als Definition formuliert, besagt, dass die Instanziierung einer klassischen Vergleichsrelation eine

1 Besonderer Dank gilt Daniel Brunnabend, der im Sommer 2018 in Bayreuth bei mir einen Kurs besuchte. Ich habe ihm wichtige Ideen zu verdanken. Einige unserer Überlegungen findet man auch in Messerli und Brunnabend (under review). 
notwendige und eine hinreichende Bedingung darstellt, damit zwei Gegenstände vergleichbar sind:

Trichotomie-These Zwei Optionen, $x$ und $y$, sind genau dann vergleichbar, wenn $x$ besser oder schlechter als $y$ oder $x$ und $y$ gleich gut sind.

Gemäss Chang ist es nun falsch, dass die Nicht-Instanziierung einer der obig genannten Vergleichsrelationen mit Unvergleichbarkeit gleichgesetzt werden kann. Das Vorliegen einer klassischen Vergleichsrelation stellt nach Chang keine notwendige Bedingung für Vergleichbarkeit dar. Damit ist gemeint, dass zwei Optionen (i) weder besser noch schlechter als noch gleich gut wie die jeweilig andere Option und (ii) dennoch vergleichbar sein können. Ergo ist gemäss Chang auch obige Definition falsch.

Bevor Changs Argument dagegen eingeführt werden kann, muss klar sein, was die Trichotomie-These meint. Auf welche Weise Chang sie interpretiert, ist jedoch nicht eindeutig rekonstruierbar. Ein Grund dafür ist, dass die Frage unbeantwortet bleibt, was unter einer Vergleichsrelation wie 'besser als' verstanden wird. Sie lässt bewusst offen, ob 'besser als' subjektivistisch oder objektivistisch zu interpretieren ist (vgl. Kapitel 2).

Chang betrachtet die Trichotomie-These als Grundlage der Entscheidungstheorie, was eher für eine subjektivistische Interpretation spricht. In der Entscheidungstheorie wird bekanntlich davon ausgegangen, dass 'besser als' mit einer Präferenz gleichgesetzt werden kann. Sie notiert: '(...) without the assumption of the Trichotomy Thesis, it is unclear how the rationality of preferences could be adequately modelled by standard utility functions.' (Chang 2002a: 666). ${ }^{3}$

Es müssen jedoch einige Annahmen erfüllt sein, damit obige Definition der Trichotomie-These gleichbedeutend mit der Axiomatik

3 Die Annahme der Vollständigkeit stellt in der heutigen (rationalen) Mikroökonomie eine notwendige Bedingung dafür dar, dass eine Präferenzrelation als Nutzenfunktion dargestellt werden kann (vgl. Mas-Collel, Whinston und Green 1995: 9). Eine Nutzen-Theorie, die ohne das Axiom der Vollständigkeit auskommt, entwickelt beispielsweise Aumann 1962. 
moderner Entscheidungstheorien ist. Ich werde dies im Folgenden kurz anhand der philosophischen Entscheidungstheorie illustrieren. Der Zweck besteht darin, eine genauere Beschreibung der Trichotomie-These zu erhalten. Um von der erstgenannten Definition der Trichotomie-These zur Axiomatik der philosophischen Entscheidungstheorie zu gelangen, sind folgende Annahmen nötig:

Erstens: 'Besser als' ist gleichbedeutend mit einer Präferenz. 'Gleich gut wie' meint eine Indifferenz.

Zweitens: Die Disjunktion (das Oder in der Definition) muss als ausschliessendes respektive exklusives Oder interpretiert werden.

Wenn diese zwei Bedingungen erfüllt sind, ist die TrichotomieThese logisch äquivalent mit spezifischen Axiomen der philosophischen Entscheidungstheorie. Das heisst, dass die Trichotomie-These wie folgt aufgeschrieben werden kann:

$(\forall x)(\forall y)(x P y \vee y P x \vee x I y)^{4}$

' $P$ ' steht für eine Präferenz, ' $I$ ' steht für eine Indifferenz, $x$ und $y$ stellen Handlungsoptionen oder Gegenstände dar. Wenn das Oder in obiger Formulierung als ausschliessendes Oder interpretiert wird, ist die Trichotomie-These logisch äquivalent zu folgenden vier Axiomen der philosophischen Entscheidungstheorie: ${ }^{5}$

$(\forall x)(\forall y)\left((x P y \vee y P x \vee x I y) \wedge(x P y \rightarrow \neg x I y) \wedge(y P x \rightarrow \neg x I y) \wedge(x I y \rightarrow(\neg x P y \wedge \neg y P x))^{6}\right.$

In der philosophischen Entscheidungstheorie wird dementsprechend angenommen, dass Optionen stets vergleichbar sind und dies so verstanden werden muss, dass in jeder Entscheidungssituation

Die Disjunktion muss als ausschliessendes Oder interpretiert werden. Ich lasse den formalen Beweis für die Richtigkeit dieser Aussage weg. Wie in der Logik üblich, muss die Disjunktion als einschliessendes Oder interpretiert werden. In der philosophischen Entscheidungstheorie wird das erste Konjunkt als connectedness condition und die anderen drei Konjunkte als asymmetry conditions bezeichnet (vgl. Kapitel 4). Das erste Axiom verlangt, dass zwei potenzielle Resultate (outputs) mithilfe der Präferenzen des Akteurs verbunden werden können. Die drei Asymmetrieaxiome verlangen, dass zwischen den einzelnen Präferenzen verschiedene Asymmetrien instanziiert sind. 
genau eine der drei Vergleichsrelationen der Trichotomie-These instanziiert ist. Die Trichotomie-These stellt ein strukturelles Axiom der Entscheidungstheorie dar. ${ }^{7}$

Damit sollte hinreichend nachvollziehbar sein, was die Trichotomie-These bedeutet.

\subsection{Changs Argumentation gegen die Trichotomie-These}

Changs Argument gegen die Trichotomie-These besteht aus folgenden zwei Schritten:

Schritt 1: Es gibt Situationen (harte Fälle), in welchen das Verhältnis zwischen zwei Handlungsoptionen nicht durch die Trichotomie-These wiedergegeben wird. Dies soll mit dem Argument der kleinen Verbesserung belegt werden.

Schritt 2: Die zwei Handlungsoptionen, welche nicht durch die Trichotomie-These zusammenhängen, sind trotzdem vergleichbar. Dies soll mit dem Ketten-Argument gezeigt werden.

Falls diese zwei Schritte richtig sind, folgt gemäss Chang - und dies ist meines Erachtens korrekt -, dass die Trichotomie-These falsch ist und dies für eine alternative Vergleichsrelation spricht. Schritt 1 respektive das Argument der kleinen Verbesserung wurde im vorletzten Kapitel besprochen. Im Folgenden wird Schritt 2 genauer diskutiert. Ich halte ihn für falsch. Das Ketten-Argument ist nicht stichhaltig, was nun gezeigt werden soll.

\subsection{Das Ketten-Argument}

Im Gegensatz zum Argument der kleinen Verbesserung weist das Ketten-Argument (chaining argument) keine bis in die 1970er Jahre zurückgehende Geschichte auf. Meines Wissens wird es von

7 Ich habe in Kapitel 4 argumentiert, dass obige Axiomatik in dem Sinne falsch ist, als Unvergleichbarkeit existiert. Chang würde obige Axiome auch ablehnen, allerdings nicht aus dem gleichen Grund, sondern deshalb, weil sich Vergleichbarkeit ihrer Meinung nach nicht in den klassischen drei Vergleichsrelationen erschöpft. 
Chang selbst eingeführt. ${ }^{8}$ Mit diesem Argument soll gezeigt werden, dass die Handlungsoptionen, welche in harten Fällen nicht durch die Trichotomie-These zusammenhängen, dennoch vergleichbar sind. Der grundlegende Gedanke im Ketten-Argument besteht aus drei Annahmen: Erstens können sehr unterschiedliche Gegenstände verglichen werden, wenn der eine Gegenstand sehr gut und der andere sehr schlecht ist. Zweitens existieren zwischen Optionen oder Gegenständen Ketten kleiner Differenzen. Drittens kann eine kleine Differenz nicht plötzlich zu Unvergleichbarkeit führen, wenn vorher Vergleichbarkeit herrschte - Chang nennt diese Idee small unidimensional difference principle. Im Wesentlichen folgert Chang allein aus diesen drei Prämissen, dass die Optionen in harten Fällen vergleichbar sind.

Chang illustriert das Ketten-Argument anhand des folgenden Beispiels: Hinsichtlich 'künstlerischen Talents' ist Michelangelo mit Mozart auf den ersten Blick, da sehr unterschiedlich, nicht vergleichbar. Mit 'auf den ersten Blick' ist gemeint, dass zwischen Mozart und Michelangelo keine der drei Relationen der Trichotomie-These besteht. Dennoch, so Chang, ist Mozart mit Michelangelo vergleichbar. Mozart hat nämlich mehr Talent als Talentlessi. Talentlessi ist ein absolut untalentierter Maler. Dies stellt die erste Prämisse dar: Der geniale Musiker hat mehr künstlerisches Talent als der untalentierte Maler. Chang spricht in diesem Zusammenhang davon, dass sogenannte Nominal-notable-Vergleiche immer möglich sind; demnach hat der notable Musiker (Mozart) mehr künstlerisches Talent als der nominal Maler (Talentlessi). ${ }^{9}$ Man kann sich nun vorstellen, dass man Talentlessi stets ein bisschen verbessert und eine Kette bildet (Talentlessi +, Talentlessi ++, Talentlessi +++, ..., Michelangelo) - daher der Name 'Ketten-Argument'. Dies stellt die zweite Prämisse dar. Am Schluss der Kette steht Michelangelo. Dabei gilt: Wenn zwei Dinge vergleichbar sind (Mozart und Talentlessi), kann eine kleine Differenz 
diese zwei Dinge nicht plötzlich unvergleichbar machen (Mozart und Michelangelo). Dies ist Prämisse drei.

Um (noch) besser nachvollziehen zu können, worum es bei diesem Argument geht, ist es sinnvoll, das Argument in einem ersten Schritt anhand eines zugänglicheren Beispiels zu veranschaulichen und in einem zweiten Schritt zu präzisieren beziehungsweise in symbolischer Sprache aufzuschreiben.

Man stelle sich vor, dass man eine offene Universitätsstelle in Philosophie besetzen muss und dabei die Wahl zwischen zwei Philosophen hat: Der eine ist sehr kreativ, aber analytisch durchschnittlich begabt. Der andere ist analytisch sehr begabt, aber durchschnittlich kreativ. Dabei möchte man denjenigen Philosophen einstellen, welcher mehr philosophisches Talent besitzt. ${ }^{10}$ Weil die unterschiedlichen Philosophen die jeweiligen Fähigkeiten in unterschiedlichem Masse besitzen, kann man nicht sagen, dass der eine Philosoph besser als der jeweilig andere ist. Gleichzeitig denkt man auch nicht, dass die beiden Philosophen gleich gut sind.

Gemäss dem Ketten-Argument sind die beiden Philosophen aber trotzdem vergleichbar. Dies soll aus folgenden beiden Prämissen folgen:

P1: Der analytisch sehr begabte, aber kreativ durchschnittliche Philosoph (notable Philosoph 1) ist besser als ein analytisch durchschnittlicher und kreativ durchschnittlicher Philosoph (nominal Philosoph respektive Talentlessi).

P2: Eine kleine Differenz kann den analytisch sehr begabten und kreativ durchschnittlichen Philosophen (notable Philosoph 1) nicht unvergleichbar machen mit dem sehr kreativen und analytisch durchschnittlich begabten Philosophen (notable Philosoph 2), wenn der analytisch sehr begabte, aber kreativ durchschnittliche Philosoph (notable Philosoph 1) vergleichbar respektive besser als ein analytisch und kreativ durchschnittlicher Philosoph (nominal Philosoph) ist.

Interessanterweise finden wir in der bisherigen Literatur keine symbolische Version des Ketten-Arguments. ${ }^{11}$

10 Das Beispiel entnehme ich aus Boot 2007.

11 Elson 2014 liefert eine präzise Rekonstruktion des Arguments, welche auf dem Mozart-Michelangelo-Beispiel basiert. 
Die abstrakte Version dieses Arguments sieht meines Erachtens wie folgt aus:

1. $x$ und $y$ sind vergleichbar;

2. es existiert eine Kette kleiner Differenzen zwischen $y$ und $c(y$, $y+, y++, \ldots, y n$ beziehungsweise $c$ );

3. wenn $x$ und das erste $y$ vergleichbar sind, dann ist $x$ mit jedem $y$ in der Kette vergleichbar, weil eine marginale Differenz nicht den Ausschlag geben kann, ob Dinge (un)vergleichbar sind

$\therefore \quad x$ und $c$ sind vergleichbar.

$x, y, c$ und $y+\ldots$ stehen dabei für Optionen oder Gegenstände.

Die Güte des Ketten-Arguments hängt von der Plausibilität der Prämissen ab. Prämisse 1 basiert auf der Idee, dass Nominalnotable-Vergleiche immer möglich sind. Prämisse 2 sagt, dass man stets Kette kleiner Differenzen zwischen zwei Gegenständen bilden kann. Prämisse 3 erklärt das small unidimensional difference principle. Formal betrachtet, funktioniert Prämisse 3 wie folgt: (i) $x$ und $y$ sind vergleichbar; (ii) wenn $x$ und $y$ vergleichbar sind, dann sind $x$ und $y+$ vergleichbar; (iii) ergo: $x$ und $y+$ sind vergleichbar; (iv) wenn $x$ und $y+$ vergleichbar sind, dann sind $x$ und $y++$ vergleichbar; (v) ergo: $x$ und $y++$ sind vergleichbar. $N$ Anwendungen von Modus ponens machen dann $x$ und $y n$ beziehungsweise $c$ vergleichbar (vgl. auch Elson 2014).

Ich denke deshalb, dass die Idee hinter dem Ketten-Argument schlicht wie folgt verstanden werden kann: $x$ und $c$ sind vergleichbar, wenn (i) $x$ und $y$ vergleichbar sind und (ii) zwischen $y$ und $c$ eine Kette kleiner Differenzen besteht.

\subsection{Bisherige Ansätze}

Es gibt einige Philosophen, welche das Ketten-Argument akzeptieren. Gemäss Carlson (2010: 120) etwa ist Changs Argument gegen die Trichotomie-These überzeugend. Andere Forscherinnen und Forscher lehnen das Ketten-Argument ab. Ich werde im Folgenden zuerst drei Einwände darstellen und danach zeigen, worin der Zusammenhang zu meinem Ansatz besteht. 
Pareto-, Sorites- und Bidirektionalität-Einwand

Alle Einwände in der Literatur beziehen sich auf Prämisse 3 beziehungsweise das small unidimensional difference principle.

\section{Pareto-Einwand}

Als Einwand gegen das Ketten-Argument könnte man die Pareto-Regel anführen. Diesen Ansatz vertritt beispielsweise Boot (2009: 81). Changs Antwort auf diesen Einwand lautet, dass das small unidimensional difference principle nur gilt, wenn die Vergleichbarkeit beziehungsweise Unvergleichbarkeit von Gegenständen nicht nach dem Pareto-Kriterium geregelt wird. Es es allerdings fragwürdig, was Changs Untersuchungsgegenstand mit dem Pareto-Kriterium zu tun haben soll. Dabei werden Dinge durcheinandergebracht. Das ParetoKriterium ist ein Effizienzkriterium, das verschiedene Verteilungszustände vergleicht. Hierbei geht es aber um mehrere Individuen und nicht um die intrapersonale Vergleichbarkeit von Handlungsoptionen (ausser man beurteilt die Auswirkungen der eigenen Handlungen nach diesem Prinzip). Ein Zustand gilt genau dann als pareto-optimal, wenn es keinen alternativen Zustand gibt, in dem mindestens eine Person besser und keine Person schlechter gestellt ist. Und: Ein Zustand S1 ist genau dann pareto-superior zu einem anderen Zustand S2, wenn mindestens eine Person in S1 besser gestellt ist als in S2 und niemand in S1 schlechter dasteht als in S2. In diesem Zusammenhang spricht man von einer Pareto-Verbesserung von S2 zu S1 (Buchanan 1988: 4). Gemäss dieser Regel kann eine kleine Differenz tatsächlich einen Unterschied machen, ob man zwei Zustände vergleichen kann oder nicht. Ein Beispiel: Die Zustände A $(9,10)$ und B $(10,9)$ sind gemäss Pareto-Regel unvergleichbar. In der Klammer stehen die Nutzen zweier Akteure in Situation A respektive B. Wenn man nun beispielsweise den Nutzen des ersten Akteurs in Situation A um eine Einheit verbessert, lautet der neue Zustand (10/10) und dieser ist vergleichbar mit Zustand B beziehungsweise pareto-besser als Zustand B. Bei der Pareto-Regel geht es also um eine gesellschaftliche Betrachtungsweise. ${ }^{12}$

12 Die Idee, die streng genommen nicht den Gedanken des Pareto-Kriteriums wiedergibt und an welche Chang möglicherweise denkt, nämlich dass z. B. eine Person mindestens eine für den Deckwert relevante Qualität besser und keine schlechter erfüllt als die andere Person, wird im übernächsten Kapitel betrachtet. 


\section{Sorites-Einwand}

Ein weiterer möglicher Kritikpunkt besteht darin zu sagen, dass Prämisse 3 ein Sorites-Argument sei, wobei es dann sinnvoll ist, davon auszugehen, dass das ganze Argument ein Sorites-Argument darstellt. Ergo würde es sich beim Ketten-Argument um eine Paradoxie handeln.

Was damit gemeint ist, lässt sich leicht nachvollziehen. Dazu müssen wir uns vergegenwärtigen, was ein Sorites-Argument darstellt und warum eine mögliche Parallele zum Ketten-Argument besteht. Es gibt verschiedene Möglichkeiten, wie ein Sorites-Argument präzise beschrieben werden kann (vgl. etwa Keefe 2007). Eine gängige Darstellung sieht wie folgt aus:

1. Fxi

2. $\forall i\left(F x_{i} \rightarrow F x_{i+1}\right)$

$\therefore$ Fxn

Ein Beispiel:

1. Ein Mann mit nur einem Haar besitzt eine Glatze.

2. Für jede Zahl $n$ gilt: Wenn eine Anzahl von $n$ Haaren eine Glatze ist, dann ist auch eine Anzahl von $n+1$ Haaren eine Glatze.

$\therefore$ Ein Mann mit extrem vielen Haaren besitzt eine Glatze.

Gemäss der zweiten Prämisse kann ein einzelnes Haar nicht ausschlaggebend dafür sein, ob jemand eine Glatze hat oder nicht. Wer die zweite Prämisse im obigen Argument bestreitet, sagt Folgendes: Es gibt eine Zahl $n$, so dass gilt: Anzahl $n$ Haare sind eine Glatze und Anzahl $n+1$ Haare sind keine Glatze. Das heisst nichts anderes, als dass ein einzelnes Haar einen Unterschied ausmachen kann, ob jemand eine Glatze hat oder nicht. Das scheint falsch zu sein. Deshalb ist die zweite Prämisse wahr. Zusammen mit der plausiblen ersten Prämisse folgt die Konklusion, dass jemand mit Anzahl $n$ Haaren (dies können extrem viele Haare sein) eine Glatze besitzt. Der Grund für diese Paradoxie ist die Vagheit des Terminus 'ist glatzköpfig. Vage Termini haben keine klare Extension (das, was unter einen Terminus fällt). Falls der Terminus 'ist glatzköpfig' eine klare Extension hätte, wäre die zweite Prämisse falsch. 
Weshalb kann man auf die Idee kommen, das Ketten-Argument könne ein Sorites-Argument sein? Prämisse 3 im Ketten-Argument sieht wie die induktive Prämisse einer Sorites-Paradoxie aus: Wie ein einzelnes Haar nicht den Unterschied machen kann, ob jemand glatzköpfig ist oder nicht, kann eine kleine Differenz nicht den Unterschied machen, ob zwei Dinge vergleichbar sind oder nicht.

Chang gibt zwei Antworten auf diesen Einwand. ${ }^{13}$ Die plausibelste Erwiderung lautet, dass der Begriff des Vergleichens kein vager Terminus ist. Falls Chang damit Recht hat, kann es sich beim Ketten-Argument um keine Sorites-Paradoxie handeln. Denn Vagheit ist notwendig dafür, dass eine Sorites-Paradoxie entstehen kann. Dass das Ketten-Argument ein Sorites-Paradox darstellt, wird denn konsequenterweise auch von denjenigen Philosophen vertreten, die Inkommensurabilität oder Unvergleichbarkeit durch die Vagheit unserer Umgangssprache erklären wollen. Die neuste Diskussion in der Literatur fokussiert auf diesen Punkt. ${ }^{14}$ Chang hingegen nennt verschiedene Gründe, weshalb der Begriff des Vergleichens nicht vage ist.

Uni-versus bidirektionale Vergleichbarkeit

Die dritte Kritik wird ebenfalls von Boot vorgetragen. ${ }^{15}$ Boots Kritik lautet wie folgt: 'Via the chain of small unidimensional delt es sich nicht um ein Sorites-Argument, weil nicht jedes Argument, das die Form eines Sorites-Arguments aufweist, auch tatsächlich eines ist. Chang nennt dafür folgendes Beispiel: ' 0 is a natural number; if $N$ is a natural number then so is $N+1$; therefore, for any $N=0,1,2,3, \ldots, N$ is a natural number.' (Chang 2002a: 680). In diesem spezifischen Punkt liegt Chang falsch. Eine sogenannte rekursive bzw. induktive Definition, wie etwa die der natürlichen Zahlen, ist kein Sorites-Argument bzw. ist darin nichts Paradoxes enthalten. Aber die Definition der natürlichen Zahlen ist überhaupt kein Argument. Es ist eine mathematische Definition. Eine solche ist eine Festlegung und kein Argument. Es gibt einen Rekursionsanfang ( 0 ist eine natürliche Zahl), einen Rekursionsschritt (Wenn $N$ eine natürliche Zahl ist, so auch $N+1$ ) und einen Rekursionsabschluss (nichts anderes ist eine natürliche Zahl). Chang geht es aber darum, dass es Argumente gibt, die wie SoritesArgumente aussehen, aber keine sind - und das kann meiner Meinung nach in diesem Kontext nur heissen, dass sie paradox aussehen, aber de facto gar nicht paradox sind. Dafür eignet sich die Definition der natürlichen Zahlen nicht als Beispiel. Denn diese sieht weder paradox aus noch stellt sie im philosophischen Sinne ein Argument dar. Vgl. etwa Constantinescu 2012 oder Elson 2014.

15 Boot 2007: 100. Diese Kritik wird in Boot 2009 nochmals erläutert und verteidigt. 
differences a significantly different situation is reached due to the directionality transition of the contributory values. This renders the chaining argument incapable of demonstrating comparability.' (Boot 2007: 103).

Was damit konkret gemeint ist, lässt sich anhand des Philosophen-Beispiels veranschaulichen, mit welchem das Ketten-Argument eingeführt worden ist: Der analytisch sehr begabte, aber durchschnittlich kreative Philosoph (notable Philosoph 1) ist besser als ein analytisch durchschnittlicher und kreativ durchschnittlicher Philosoph (nominal Philosoph respektive Talentlessi). Dies ist unproblematisch, um Boots Terminologie zu gebrauchen, da dieser Vergleich unidirektional ist. Damit ist gemeint, dass der notable Philosoph 1 bei keinem für den Vergleich relevanten Wert schlechter und in mindestens einem Wert besser abschneidet: Er ist analytischer als und mindestens gleich kreativ wie der nominal Philosoph (ein ähnliches Konzept wie dasjenige der Pareto-Vergleichbarkeit).

Das Problem besteht gemäss Boot nun darin, dass die Vergleichbarkeit nicht aufrechterhalten wird, wenn aus einem unidirektionalen ein bidirektionaler Vergleich wird. Dass heisst in unserem Beispiel, dass der notable Philosoph 1 nicht mehr in sämtlichen für den Vergleich relevanten Werten besser abschneidet als der notable Philosoph 2: Der eine ist kreativer und der andere analytischer. Boot würde Chang wohl zugestehen, dass Prämisse 2 des Kettenarguments zutrifft und eine Kette der Art $y, y+, y++, \ldots, c$ besteht. Dabei steht $y$ in unserem Beispiel für den nominal Philosophen und $c$ für den notable Philosophen 2. Der Faktor, der dabei verändert wird, ist eine marginale Erhöhung der philosophischen Kreativität. Das Problem entsteht aber genau dann, wenn irgendein $y$ in der Kette plötzlich kreativer als notable Philosoph $1(x)$ ist - bei gleichzeitiger Unterlegenheit, was die analytischen Fähigkeiten betrifft. Das ist, was Boot meint, wenn er schreibt: 'Paradoxically, a one-dimensional change creates a two-dimensional problem of comparison.' (Boot 2009: 78). Das Mozart-Michelangelo-Beispiel ist gemäss Boot in dieser Hinsicht lediglich nicht eindeutig klar formuliert respektive würde sich obiges Problem auch ergeben, wenn darin ein solcher Wechsel von einem uni- zu einem bidirektionalen Vergleich stattfände. 


\section{Zusammenhang}

In welchem Verhältnis steht dieses Kapitel zur bisherigen Forschung? Ich werde auch argumentieren, dass Prämisse 3 beziehungsweise das small unidimensional difference principle falsch ist. Aber ich werde einen neuen Ansatz entwickeln, welcher auf Nominal-notableVergleichen und unpräzisen Entscheidungskriterien basiert. Die bisherige Forschung hat nicht berücksichtigt, dass sich die Vagheit auf das Entscheidungskriterium selbst beziehen kann. Zudem bin ich mit Boot einverstanden, dass in der Kette ein Wechsel von Vergleichbarkeit zu Unvergleichbarkeit stattfinden kann. Mein Ansatz liefert jedoch eine genauere Begründung, weshalb das so ist. Boot liegt falsch: der Grund kann nicht darin bestehen, dass ein Wechsel von einem One-dimensional- zu einem Two-dimensional-Vergleich stattfindet, weil eine Abwägung (trade-off) zwischen verschiedenen Aspekten wie analytisch und kreativ möglich ist.

\subsection{Ein Gegenbeispiel}

Es kann in der Kette einen Wechsel von Vergleichbarkeit zu Unvergleichbarkeit stattfinden. Der Grund ist, dass eine marginale Veränderung eines Faktors zwar nicht unbedingt an einem bestimmten Punkt, aber sehr wohl in der Summe einen Einfluss auf die Vergleichbarkeit beziehungsweise Unvergleichbarkeit haben kann. Ich versuche dies im Folgenden anhand zweier Schritte zu zeigen: Erstens illustriere ich anhand eines Beispiels, weshalb die Addition vieler unwesentlicher einzelner Faktoren einen Unterschied in der Bewertung von Optionen ausmachen kann. Zweitens wird ein Beispiel geschildert, in dem die Addition vieler kleiner Faktoren einen Unterschied ausmachen kann, ob zwei Optionen vergleichbar sind oder nicht.

Nehmen wir an, dass eine Million Franken für einen bestimmten Akteur besser als ein kaum spürbarer Stromschlag ist. Und nehmen wir an, dass eine Million Franken für denselben Akteur schlechter als ein tödlicher Stromschlag ist. Dieser Akteur macht nun als Proband in einem Experiment mit, in der man zuerst die Wahl zwischen einem kaum spürbaren Stromschlag und einer Million Franken hat. Dabei wählt er das Geld. Im Folgenden wird dasselbe Experiment 
immer wieder wiederholt, mit dem Unterschied, dass in jeder Runde die Stromstärke marginal - so, dass es für den Akteur nicht spürbar ist - erhöht wird. Das heisst, dass die Erhöhung der Stromstärke zwischen zwei Runden für die Bewertung keinen Unterschied ausmacht. In der Summe ist die Erhöhung der Stromstärke aber spürbar respektive tödlich und kann zu einem Wechsel der Bewertung führen. Auch wenn der Akteur also nicht präzise angeben kann, in welcher Runde seine Präferenzen wechseln, kann die Addition vieler marginaler Veränderungen einen Unterschied in der Bewertung bewirken.

Analog kann die Addition vieler unwesentlicher einzelner Faktoren eine Rolle spielen, ob zwei Optionen für einen Akteur vergleichbar oder unvergleichbar sind. Man stelle sich vor, dass eine Akteurin nicht sagen kann, ob sie Anwältin oder Musikerin werden will. Damit ist gemeint, dass sie keinen der beiden Berufe als besser als den jeweilig anderen bewerten kann und sie gleichzeitig auch nicht für gleich gut hält. In diesem Fall sind die Optionen für die Akteurin unvergleichbar. Gleichzeitig ist eine Anwaltskarriere, in der man nach zehn Jahren so viel verdient hat, dass man mit dem Arbeiten aufhören kann, für dieselbe Akteurin besser als der Beruf als Musikerin. In diesem Fall sind die Optionen für die Akteurin vergleichbar. Der einzige Faktor, der sich dabei verändert hat, so die Annahme, ist die Erhöhung des Lohnes.

So wie die Erhöhung der Stromstärke in der Summe spürbar respektive tödlich ist und zu einem Wechsel der Bewertung führen kann, kann eine Verbesserung eines beliebigen Faktors wie beispielsweise die Erhöhung des Lohnes in der Summe zu einer Änderung in der Bewertung führen. Der einzige Unterschied in den beiden Beispielen besteht darin, dass der Akteur im Experiment zu Beginn eine klare Bewertung der Situation vornehmen kann (eine Million ist besser als ein kaum spürbarer Stromschlag) und die Akteurin im Berufsexempel nicht. Aber dies ändert nichts daran, dass eine Verbesserung eines einzelnen Faktors in der Summe einen Unterschied in der Bewertung ausmachen kann - unabhängig davon, ob die Optionen zu Beginn besser als die jeweilig andere Option oder unvergleichbar sind. Man könnte einwenden, es stelle eine unrealistische Annahme dar, dass die Erhöhung des Lohnes der einzigen Faktor sei, der sich verändert. Schliesslich geht dies ja auch mit anderen Dingen einher wie beispielsweise, dass man seine Kinder öfters sehen kann. Oder es ist denkbar, dass das zusätz- 
liche Geld ein Mittel darstellt, unerfüllte Träume zu verwirlichen, wie etwa nach Frankkreich auszuwandern oder eine Weltreise zu machen.

Es mag sein, dass das Berufsbeispiel in dieser Hinsicht schlecht gewählt ist. Aber man kann sich vorstellen, dass das beschriebene Stromschlagexperiment in einer Runde beginnt, in der die Optionen für den Akteur weder besser noch schlechter als noch gleich gut sind. Das Schmerzniveau kann beispielsweise ein Level aufweisen, bei dem der Akteur nicht weiss, ob er die Schmerzen für die Million aushalten soll oder nicht. In diesem Fall würde auch in diesem Beispiel irgendwann - mit zunehmender Steigerung der Stromstärke - ein Wechsel von Unvergleichbarkeit zu Vergleichbarkeit stattfinden, wobei die marginale Erhöhung der Stromstärke den einzigen Faktor darstellt, der sich verändert. Wo, wenn nicht im Versuchslabor, kann diese von Chang geforderte Ceteris-paribus-Bedingung eingehalten werden?

Es ist klar, dass das Gegenbeispiel ein bestimmtes Konzept von (Un)Vergleichbarkeit voraussetzt. Es wird von einem Ansatz ausgegangen, wie er in Kapitel 2 und 3 entwickelt worden ist. Gemäss dieser Sichtweise hat (Un)Vergleichbarkeit letztlich mit der Bewertung von Gründen und Werten zu tun, die für oder gegen die zur Auswahl stehenden Optionen sprechen. Hierbei ist es möglich, dass die marginale Veränderung eines einzelnen Faktors eine Änderung in den Gründen und somit auch eine Änderung in den Präferenzen zur Folge haben kann: Ein leichter Stromschlag stellt keinen Grund dar, auf eine Million zu verzichten, ein tödlicher Stromschlag hingegen wohl. Ein höheres Einkommen stellt keinen Grund dar, den Beruf des Anwalts gegenüber demjenigen des Musikers zu präferieren. Ein so hohes Einkommen, dass nach zehn Jahren nicht mehr gearbeitet werden muss, stellt einen Grund dar, den Beruf des Anwalts gegenüber demjenigen des Musikers zu präferieren.

Ein wichtigerer Einwand lautet deshalb, dass das Gegenbeispiel nicht mit jedem Konzept von Vergleichbarkeit funktioniert. Diese potenzielle Kritik wird im nächsten Abschnitt betrachtet.

\subsection{Mögliche Kritik}

Man kann argumentieren, dass die Gegenbeispiele gemäss Changs Interpretation eines harten Falles nicht funktionieren. Chang 
würde vermutlich sagen, dass die Beispiele nur einen Wechsel von 'besser als' zu 'Parität' demonstrieren.

Natürlich könnte man gegen Chang einwenden, dass das Ketten-Argument nicht Parität voraussetzen sollte. Im Gegenteil: Das Ketten-Argument sollte Parität etablieren. Aber man könnte gegen uns ebenso argumentieren, dass wir nicht ein bestimmtes Konzept von Vergleichbarkeit voraussetzen sollten. Aus diesem Grund werde ich im nächsten Abschnitt eine neue Theorie entwickeln. Inwiefern kann damit die potenzielle Kritik entkräftet werden? Jeder wird akzeptieren, dass das small unidimensional difference principle nur gilt, wenn man eine Abwägung zwischen den verschiedenen Aspekten vornehmen kann - das ermöglicht Nominal-notable-Vergleiche. Gleichzeitig wird ersichtlich werden, weshalb eine Serie von marginalen Veränderungen einen Wechsel von 'eine Abwägung ist möglich' zu 'eine Abwägung ist unmöglich' bewirken kann. Anders formuliert: Ich zeige, wie Chang Nominal-notable-Vergleiche begründen kann, verwende den Begriff der (Un)Vergleichbarkeit neutraler und auch in Changs Sinne - eine Abwägung ist (un)möglich) - und belege, wie es zu einem Wechsel von Vergleichbarkeit zu Unvergleichbarkeit kommen kann.

\subsection{Vage Entscheidungskriterien}

Die in Abschnitt 7.4 beschriebene Situation kann mithilfe der folgenden Matrix abgebildet werden:

\begin{tabular}{c|c|c} 
Option/Dimension & Geld & Freude $(+) /$ Schmerz $(-)$ \\
\hline Leichter Stromschlag + 1M Fr. $(y)$ & 10 & -10 \\
\hline Verletzung + 1M Fr. $(y----)$ & 10 & -100 \\
\hline Tod +1M Fr. $\left(y_{n}\right)$ & 10 & 0 \\
\hline Das Experiment stoppen $(x)$ & 0 & 0
\end{tabular}

Nutzen stellt in diesem Beispiel ein plausibles Entscheidungskriterium beziehungsweise einen Deckwert $(V)$ dar. Ich denke wohlgemerkt nicht, dass Vergleichbarkeit solche Deckwerte voraussetzt (vgl. Kapitel 1). Aber einerseits stellt das Changs Position dar. Und andererseits ist es in den relevanten Beispielen in diesem Kapitel 
nicht unplausibel, dass solche Entscheidungskriterien zur Verfügung stehen. Deshalb orientiere ich mich hierbei an Chang. Die Ziffern entsprechen dem Wert für Geld, Freude und Leid in Bezug auf Nutzen. Ich werde gleich darauf zu sprechen kommen, wie wir diese Zahlen interpretieren können.

Wie in Abschnitt 7.4 dargelegt wurde, ist ein kaum spürbarer Stromschlag plus eine Million Franken (Option x) für viele Akteure wertvoller, als das Experiment abzubrechen (Option $y$ ). Diese beiden Optionen sind demnach vergleichbar. Aber welche Annahmen brauchen wir, um zu dieser Konklusion zu gelangen? In der bisherigen Literatur basiert die Plausibilität von Nominal-notable-Vergleichen auf Intuition. Für unsere Zwecke ist es jedoch wichtig, die zugrundeliegenden Annahmen explizit zu machen. Ich denke, dass der relevante Nominal-notable-Vergleich auf mindestens zwei Prämissen basiert:

1. Geld kann Schmerzen kompensieren. Abwägungen sind möglich.

2. Die Schmerzeinheit -1 überwiegt die Geldeinheit 10 nicht. (Das Verhältnis in $V$ ist gleich/kleiner als $90.9 \%$. $)^{16}$

Die erste Annahme ist unstrittig. Chang (2002: 676) selbst argumentiert: 'The principle [small unidimensional difference principle] presupposes that the comparability of two evaluatively very different items is a matter of balancing or trading off the way one relevant respect is borne against the way another relevant respect is borne.' (Die relevanten Dimension ('respects') sind dabei Geld und Schmerz.)

Die zweite Prämisse besagt, dass wir ein intuitives Konzept des Deckwerts beziehungsweise Entscheidungskriteriums besitzen (ein grobes Konzept von Nutzen in diesem Kontext), das es uns erlaubt, solche Urteile zu fällen. Aber wie präzise kann dieses Konzept sein? Wie kann man erklären, dass wir $x$ und $y$ vergleichen können und gleichzeitig ein Wechsel von 'eine Abwägung ist möglich' zu 'eine Abwägung ist nicht möglich’ stattfindet? Um diese Fragen beantworten zu können, müssen wir uns das Entscheidungskriterium genauer anschauen. 
In unserem Beispiel ist Nutzen schlicht zu wenig präzise definiert. Das Entscheidungskriterium ist vage. Damit ist gemeint, dass es für uns aufgrund unserer kognitiven Fähigkeiten nicht möglich ist, hinsichtlich des Entscheidungskriteriums eine präzise Abwägung vorzunehmen. Präzise könnte etwa heissen, eine ratio scale zu benützen (ich komme darauf zurück). Der Vergleich zwischen $y$ und $x$ ist jedoch möglich, weil in diesem Fall ein vages Kriterium ausreicht. Das heisst, dass innerhalb der Kette (von y zu $y-----$ ) folgendes passiert: Der Grad der Präzision in der Definition von $V$ muss immer genauer werden, damit ein Vergleich mit $x$ möglich ist.

Im Vergleich zwischen $y$ und $x$ ist es ausreichend, dass die Verhinderung von Schmerz sicher nicht $10 \mathrm{mal}$ wichtiger als Geld ist. Oder was äquivalent ist: Das Gewicht von Freude und Schmerz in $V$ ist sicherlich gleich/kleiner als $90.9 \%$.

Um zu sehen, weshalb diese Gleichsetzung gilt, können wir die Werte von $y$ und $x$ betrachten. In Option $y$ erhält man einen leichten Stromschlag (-1) und eine Million Franken (10). In Option $x$ ist man keinem Stromschlag ausgesetzt (0) und erhält kein Geld (0). Wenn das Gewicht von Schmerz und Freude kleiner/gleich $90.9 \%$ ist, dann ist der Wert von $y$ grösser als der Wert von $x$. Ein Beispiel: Nehmen wir an, dass das Gewicht von Schmerz und Freude in $V$ genau $90.9 \%$ ist. In diesem Fall ist der Wert von $y 0.1(-1 \times 90.9+10 \times 9.1) .0 .1$ ist grösser als 0 und $y$ dementsprechend besser als $x$. Wenn nun aber das Gewicht von Schmerz und Freude in $V$ grösser als $90.9 \%$ ist, dann ist der Wert von $x$ grösser als der Wert von $y$. Ein Beispiel: Nehmen wir an, dass das Gewicht von Schmerz und Freude in $V 91 \%$ ist. In diesem Fall ist der Wert von $y-1(-1 \times 91+10 \times 9)$. 0 ist grösser als -1 und $x$ dementsprechend besser als $y$.

Betrachten wir nun den Fall, dass eine Akteurin die Intuition hat, dass Geld und die Verhinderung von Schmerz etwa gleich wichtig sind. Wenn die Akteurin zwischen $y$ und $x$ wählt, ist $y$ klar besser. Der Grund ist, dass der Wert von $y(10 \times 50-1 \times 50=450)$ grösser ist als der Wert von $x(0)$.

Und nun der entscheidende Punkt: Die Intuition hat dabei eine erlaubte Fehlerquote von mehr als $40 \%$. Anders formuliert: Selbst wenn die Intuition $40 \%$ daneben liegt und das Gewicht von Freude und Schmerz in $V 90 \%$ ist, wäre das Urteil, dass $y$ besser als $x$ ist, 
immer noch richtig. Um zu sehen, weshalb das so ist, können wir annehmen, dass das Gewicht von Freude und Schmerz in $V 90 \%$ ist. In diesem Fall ist $y$ besser als $x$, weil der Wert von $y(10 \times 10-1 \times 90=10)$ grösser als der Wert von $x(0)$ ist.

Wenn die Akteurin allerdings zwischen $y-----(10 \mid-8)$ und $x$ wählen muss, ist der Fall nicht mehr so eindeutig. Klar: Der Wert von $y(10 \times 50-8 \times 50=100)$ ist grösser als der Wert von $x(0)$. Aber die Intuition hat eine erlaubte Fehlerquote von weniger als $6 \%$. Anders formuliert: Wenn die Intuition $6 \%$ daneben liegt, und das Gewicht von Freude und Schmerz in $V 56 \%$ ist, wäre das Urteil falsch, dass $y$ besser als $x$ ist. Um zu sehen, weshalb das so ist, können wir annehmen, dass das Gewicht von Freude und Schmerz in $V 56 \%$ ist. In diesem Fall ist $x$ besser als $y$, weil der Wert von $x(0)$ grösser als der Wert von $y$ (44 $\times 10-56 \times 8=-8)$ ist. Im Vergleich zwischen $y-----(10 \mid-10)$ und $x$ liegt die erlaubte Fehlerquote bei $0 \%$. Die Akteurin kann deshalb nicht sagen, ob $y-----$ und $x$ besser, schlechter oder gleich gut sind.

Man mag einwenden, dass $x(0 \mid 0)$ und $y-----(10 \mid-10)$ gleich gut sind. Aber das ist falsch. Nutzen spezifiziert nicht den exakten Geldbetrag, der nötig ist, um ein bestimmtes Niveau an Schmerzen zu kompensieren. Der Wert 1 in der Spalte Geld hat nicht exakt den gleichen Wert wie der Wert 1 in der Spalte Freude/Schmerz. Anders formuliert: Die Option (1|-1) hat nicht einen exakt neutralen Wert. Solche Abwägungen sind aus ganz verschiedenen Gründen zu schwierig für Menschen, wobei ich eine mögliche Erklärung liefere, weshalb das in unserem Beispiel der Fall ist: Das Konzept des Nutzens lässt nur unpräzise Urteile zu. ${ }^{17}$

Es ist in diesem Zusammenhang wichtig zu sehen, dass auch Chang nicht sagen kann, dass die Option (1|-1) einen exakt neutralen Effekt hat. Der Grund ist, dass dann alle Optionen entweder besser, schlechter oder gleich gut wären - eine Wertrelation, die sie bereits ausgeschlossen hat. und Geld zusammenhängen. Es scheint sich so zu verhalten, dass wir in unserem Beispiel mehr als eine rein ordinale Rangordnung angeben können (normalerweise ist das jedoch eine zu starke Annahme). Wir können folgende drei Optionen betrachten, um zu sehen, weshalb das in unserem Fall so ist: (10|-1), (10|-10), (10|-100). (10|-1) ist klar besser als (10|-10), aber (10|-10) ist viel besser als (10|-100). Das heisst: Die Distanz zwischen $(10 \mid-10)$ und (10|-100) ist grösser als die Distanz zwischen (10|-1) und (10|-10). 
Um meine Argumentation zu stärken, wollen wir noch ein anderes Beispiel näher betrachten, den Mozart-Michelangelo-Vergleich. Dieses Beispiel scheint wie folgt zu funktionieren:

\begin{tabular}{c|c|c} 
Option/Dimension & Musik & Bildhauerei \\
\hline Mozart & 10 & 0 \\
\hline Michelangelo & 0 & 10 \\
\hline Talentlessi & 0 & 1
\end{tabular}

Gemäss Chang ist Talentlessi klar schlechter als Mozart (in Bezug auf Kreativität). Das scheint plausibel zu sein. Aber welche Annahmen brauchen wir, um zu dieser Konklusion zu gelangen? Es scheinen wieder die folgenden zwei zu sein: (1) Abwägungen zwischen beiden Dimensionen sind möglich. (2) Der höhere Wert von Talentlessi in der Dimension Bildhauerei kann den Wert von Mozart in der Spalte Musikalität nicht übertrumpfen (der Anteil von Bildhauerei in $V$ ist kleiner als 90.9\%). Die zweite Annahme besagt, dass wir ein vages Entscheidungskriterium oder Konzept von Kreativität besitzen, welches es uns erlaubt, solche Urteile zu fällen.

Nehmen wir nun vereinfachend an, dass Musik und Bildhauerei ungefähr gleich wichtig für Kreativität sind. Ist damit gemeint, dass das Gewicht exakt 50:50 ist? Nochmals: Das kann nicht der Fall sein, weil dann alle Dinge anhand von klassischen Vergleichsrelationen verglichen werden könnten. Mozart und Michelangelo wären genau gleich kreativ. Das Argument der kleinen Verbesserung könnte nicht angewendet werden.

Wie also können wir den Nominal-notable-Vergleich (Talentlessi|Mozart) und das gleichzeitige Versagen der Trichotomie-These zwischen Michelangelo und Mozart erklären?

Wir müssen den Deckwert genauer betrachten. Unser Konzept von Kreativität ist schlicht zu wenig präzise definiert. Daraus entstehen Situationen, in denen kein Vergleich möglich ist. Nominalnotable-Vergleiche sind aber möglich, weil hierbei ein vages Konzept des Deckwerts ausreicht, um ein Urteil fällen zu können. Das heisst, dass innerhalb der Kette (von Talentlessi zu Michelangelo) folgendes passiert: Der Grad der Präzision in der Definition von $V$ muss immer genauer werden, damit ein Vergleich mit Mozart möglich ist. 
Betrachten wir etwa den Vergleich zwischen Talentlessi++++ (0|8) und Mozart (10|0). Um das Urteil fällen zu können, dass Mozart kreativer als Talentlessi++++ ist, gilt Folgendes: Die Intuition, dass Musikalität und Bildhauerei gleich wichtig sind in Bezug auf Kreativität, hat eine Fehlerquote von weniger als $6 \%$. Im Vergleich zwischen Talentlessi (0|1) und Mozart hingegen ist die Fehlerquote grösser als $40 \%$.

Halten wir fest: Es sollte klar geworden sein, weshalb eine marginale Veränderung eines Faktors ein Wechsel von 'eine Abwägung ist möglich' zu 'eine Abwägung ist nicht möglich' zur Folge haben kann, auch wenn wir nicht sagen, wann genau das passiert. Aber das sollte niemanden überraschen. Dinge hinsichtlich vager Entscheidungskriterien zu vergleichen, stellt keine exakte Wissenschaft dar.

\subsection{Zusammenfassung}

In einem ersten Schritt wurde die Trichotomie-These eingeführt. In einem zweiten Schritt wurde Changs Argumentation rekonstruiert, weshalb die Trichotomie-These falsch ist. In einem dritten Schritt wurde das Ketten-Argument anhand einfacher Beispiele veranschaulicht und präzisiert. In einem vierten Schritt wurden bestehende Einwände gegen das Ketten-Argument diskutiert, dann wurde dargelegt, wie unser Ansatz damit zusammenhängt. In einem fünften Schritt wurde ein Gegenbeispiel gegen das Ketten-Argument eingeführt. In einem sechsten Schritt wurde eine potenzielle Kritik daran erörtert. Aus diesem Grund wurde letztlich eine neue Theorie entwickelt, die auf vagen Entscheidungskriterien basiert.

In diesem Kapitel wurde also gezeigt, dass Changs Argumentation gegen die Trichotomie-These nicht haltbar ist. Daraus folgt aber nicht unbedingt, dass es keine weitere Vergleichsrelation gibt. Chang könnte Recht haben, dass es eine vierte Vergleichsrelation namens 'Parität' gibt; sie könnte mit dem Ketten-Argument lediglich ein falsches Argument benützen, um dies zu belegen. Die Möglichkeit, dass harte Fälle nicht als Unvergleichbarkeit, sondern als 'Parität' gedeutet werden können, sollte in Betracht gezogen werden. Darum geht es im nächsten Kapitel. 
Kapitel 8

Parität 
Im letzten Kapitel wurde gezeigt, dass Changs Argumentation gegen die Trichotomie-These nicht stichhaltig ist. In diesem Kapitel steht die Frage im Mittelpunkt, ob sich die Handlungsoptionen in harten Fällen mithilfe des Konzepts der Parität trotzdem vergleichen lassen. Es ist denkbar, dass Chang ein unplausibles Argument gegen die Trichotomie-These erwähnt und man die Rationalität dennoch auf der Ebene der Wertrelationen retten kann.

Um diese Frage beantworten zu können, stelle ich zuerst Changs Überlegungen dar. Danach wird Gerts Interpretation von Parität diskutiert. Schliesslich wird der Frage nachgegangen, ob harte Fälle mit den unterschiedlichen Deutungen von Parität verglichen und aufgelöst werden können. Ich argumentiere, dass dies nicht gelingt.

Changs Ausführungen zum Begriff der Parität, verstehe ich sie richtig, stellen nichts anderes als eine sprachliche Umformulierung der Aussage dar, dass es harte Fälle gibt. Gerts Interpretation von $\mathrm{Pa}$ rität basiert auf der Gleichsetzung einer Präferenz und einer Wahl, was keine adäquate Charakterisierung eines harten Falles darstellt.

Beide Interpretationen verkennen einen wichtigen Zweck von Vergleichsrelationen, nämlich, dass sie eine einfache Ordnung zwischen den Optionen herstellen und auf diese Weise einen Übergang von den möglichen zu gewählten Alternativen gewährleisten können.

Es ist wichtig zu erwähnen, dass ich nicht alle Interpretationen von Parität in der Literatur diskutiere. ${ }^{18}$

Dementsprechend bleibt die Frage offen, ob in harten Fällen mithilfe alternativer Deutungen Vergleichbarkeit hergestellt werden kann. Changs Ausführungen betrachte ich, weil sie das Konzept einführt. Gerts Deutung diskutiere ich aus dem Grund, dass viele weitere Interpretationen von Parität auf Gerts Modell aufbauen (vgl. etwa Rabinowicz 2008). pigkeit aufweist, welche ich im nächsten Kapitel diskutiere. Ich will aber nicht sagen, dass Andreous Deutung keine eigene Diskussion wert wäre. Auch Yitzhaks Interpretation von Parität spielt in diesem Kapitel keine Rolle. Ein Grund dafür ist, dass Yitzhaks semantische und formale Überlegungen, welche er auf das Mozart-Michelangelo-Beispiel oder Prädikate wie 'ist glatzköpfig' anwendet, für Entscheidungssituationen im Alltag - diejenigen harten Fälle, die in dieser Arbeit interessieren - irrelevant zu sein scheinen (vgl. Yitzhak 2009). 


\subsection{Changs Ausführungen zu Parität}

Chang hat nicht den Anspruch, eine Definition von Parität zu liefern. Gleichwohl finden wir bei ihr einige Ausführungen dazu. In Making Comparisons Count fragt Chang: 'What is this fourth relation?' Und sie antwortet: 'Since, like better than, on a par is primitive, no analysis can be given. But we might try to elucidate the concept.' (Chang 2002b: 141). Zu diesem Zweck greift Chang auf einige Ausführungen zurück, die sie erstmals im Vorwort zu Incommensurablity, Incomparability, and Practical Reason formuliert hat: 'The core idea of parity can be approached by focusing on the idea of an evaluative difference with respect to a covering value. Where there is some evaluative difference between two items, that difference is (1) zero or nonzero, and (2) biased or unbiased. A difference is zero if it does not have extent. A difference is biased if it favors one item and, correspondingly, disfavors the other.' (Chang 1997: 26).

Die Vergleichsrelationen der Trichotomie-These können gemäss Chang nun folgendermassen erklärt werden: Wenn eine Differenz zwischen zwei Gegenständen nicht null und biased (einseitig befangen) ist, dann ist einer der Gegenstände besser als der andere. Wenn die Differenz null ist und deshalb unbiased, dann sind die beiden Gegenstände gleich gut. Nach Chang gibt es aber auch sogenannte nonzero, unbiased Differenzen. Falls dies der Fall ist, besteht die Beziehung der Parität. ${ }^{19}$ Als Beispiel für eine solche Differenz nennt sie den Unterschied zwischen zwei verschiedenen Routen bezüglich benötigter Zeit, um an einen bestimmten Ort zu kommen: 'We might want to know the unbiased difference in the time it takes to get to London by two different routes.' (Chang 1997: 26). Auch die Beziehung zwischen Mozart und Michelangelo oder die Wahl zwischen unterschiedlichen Berufen sind gemäss Chang Beispiele für solche nonzero, unbiased Differenzen.

Ich verstehe Chang wie folgt: Die Beziehung der Parität besteht dann, wenn eine Option weder besser noch schlechter als noch gleich gut wie eine andere Option ist. Die beiden Optionen sind nicht gleich gut, weil der Unterschied zwischen ihnen nicht null ist. Da es aber 
einen Unterschied zwischen ihnen gibt, demgegenüber wir nicht indifferent sind, und zugleich keine Option gegenüber der anderen bevorzugt wird, besteht zwischen ihnen die Beziehung der Parität. Parität, so kann man sagen, ist eine Form nicht gleichgültiger Differenz, die jedoch keine der Optionen als besser oder schlechter auszeichnet. Ich bin allerdings unsicher, wie dies genauer verstanden werden muss und kann Carlsons Kommentar nachvollziehen, dass Changs Erläuterungen nicht hilfreich sind, um die Bedeutung des Begriffs der Parität erfassen zu können (Carlson 2010: 122).

Grundsätzlich ist es plausibel, dass zwischen zwei Optionen manchmal in dem Sinne eine Differenz besteht, dass wir keine der beiden Optionen bevorzugen und gleichzeitig nicht indifferent zwischen ihnen sind (im Sinne einer Gleichgültigkeit). Es ist aber nicht nachvollziehbar, welche zusätzlichen Informationen uns Chang bei der Explikation von Parität zur Verfügung stellt. Dasjenige, was Chang als Parität beschreibt, scheint nichts anderes als die Beschreibung eines harten Falles zu sein. Betrachten wir im Folgenden deshalb Gerts genauere Deutung von Parität.

\subsection{Gerts Interpretation von Parität}

Ich erläutere zuerst Gerts These: Parität ist keine vierte Vergleichsrelation. Zweitens wird Gerts Theorie rekonstruiert, welche diese These etablieren soll. Um Gerts Interpretation von Parität nachvollziehen zu können, müssen die Grundannahme und der Formalismus der Theorie verstanden werden. Drittens wird gezeigt, dass Gert seine Theorie nicht korrekt auf Changs Idee anwendet, und erläutert, wie Gert innerhalb seiner Theorie Parität erklären kann/sollte. Viertens werden Schwierigkeiten von Gerts Ansatz dargestellt.

\section{Die These}

Bezüglich des Ziels seines Aufsatzes ‘Value and Parity’ hält Gert Folgendes fest: 'This article will defend the trichotomy thesis at least in one important sense: it will hold that any other positive value relations that we might wish to make use of can be defined in terms of 
the three traditional relations.' (Gert 2004: 493). Gert kritisiert Chang dahingehend, dass es keine vierte Vergleichsrelation namens Parität gibt. Jede Vergleichsrelation kann gemäss Gert auf eine der drei Beziehungen der Trichotomie-These reduziert werden. ${ }^{20}$ Auf jeden Fall liefert Gert, wenn auch nicht in Changs Sinne, eine eigene Interpretation des Konzepts der Parität.

\section{Die Theorie}

Die Grundannahme von Gerts Theorie besagt, dass es einen Unterschied zwischen dem Begriff der Präferenz und dem Begriff des Werts gibt: 'That is, preference and valuation are not the same thing, and preference is most closely related to choice, while valuation is more closely related to the assessment of choice.' (Gert 2004: 493). Gert geht davon aus, dass eine Präferenz durch eine Wahl zum Vorschein kommt, während ein Wert durch eine Festsetzung offengelegt wird, welche Wahlen man als zulässig betrachtet. Er spricht in diesem Zusammenhang von to be revealed (Gert 2004: 493). Es ist nicht eindeutig, was Gert unter closely related und to be revealed versteht. Vereinfacht können wir aber Folgendes sagen: Eine Präferenz ist bei Gert eine Wahl. Ein Wert ist bei Gert eine Festsetzung, welche Wahlen zulässig sind.

Hinter dieser Unterscheidung steckt gemäss Gert die Intuition, dass wir Entscheidungen, die anders ausfallen als unsere, trotzdem als rational zulässig betrachten können, wobei es Grenzen gibt, ob wir das tun oder nicht. Weiter, so Gert, kann mit der Unterscheidung folgendes Phänomen erklärt werden: $\mathrm{Oft}$, wenn wir $A$ gegenüber $B$ präferieren, denken wir nicht, dass A besser als $B$ ist. ' $A$ ist besser als $B$ ' bedeutet bei Gert, dass es ein Fehler wäre, wenn man $A$ nicht gegenüber $B$ präferieren würde (Gert 2004: 499). Man kann sich vorstellen, dass man das Studium der Philosophie gegenüber dem Studium der

20 Inwiefern Parität in Gerts Modell tatsächlich eine Reduktion auf die klassischen Vergleichsrelationen darstellt, wurde allerdings bestritten (Rabinowicz 2008). Diese Kritik ist plausibel. Deshalb, weil zwischen zwei Optionen, $A$ und $B$, gemäss Gerts Modell genau dann die Relation der Parität besteht, wenn diese nicht traditionally comparable sind. Dies heisst, dass zwischen $A$ und $B$ keine der Vergleichsrelationen der Trichotomie-These instanziiert ist. Was damit gemeint ist, kann an dieser Stelle noch nicht nachvollzogen werden. Es wird später in diesem Abschnitt erklärt. 
Jurisprudenz bevorzugt, aber denkt, dass es auch zulässig gewesen wäre, wenn man sich umgekehrt entschieden hätte.

Der Formalismus der Theorie lautet folgendermassen:

\begin{tabular}{c|c} 
Präferenz & Wert \\
\hline Wahl & Welche Wahl rational erlaubt ist \\
\hline 4 & $(3,5)$
\end{tabular}

Die Zahlen, welche in obiger Grafik völlig willkürlich gewählt sind, entsprechen den Stärken der Präferenzen. In der Klammer $(3,5)$ steht der Bereich der erlaubten Stärken.

Implizit suggeriert Gert mit dieser Idealisierung, dass Präferenzen quantitativ messbar sind. Gerts Bezeichnung strength of preference legt nahe, dass es darum geht, wie stark oder fest man etwas will - im Vergleich zu etwas anderem. Je höher die Zahl, desto stärker will man dieses und nicht jenes. Es ist meines Erachtens allerdings schlicht falsch, dass wir Präferenzen genaue Zahlen zuordnen können.

Gerts Idealisierung sollte am sinnvollsten auf diese Weise gedeutet werden, dass Präferenzen ordinal interpretiert werden. ${ }^{21}$ Ein Beispiel: Eine Präferenzstärke der Zahl 10 für 'Dissertation schreiben' und eine Präferenzstärke der Zahl 5 für 'ins Schwimmbad gehen' sollte so interpretiert werden, dass man es vorzieht, an der Dissertation zu arbeiten.

Die Ränge hingegen widerspiegeln die Grenzen, ob man eine Wahl als rational zulässig betrachtet oder nicht. Dies bedeutet, dass die Ränge den Werten entsprechen. Ein Beispiel: Nehmen wir an, dass sich ein Akteur überlegt, ins Kino zu gehen. Gerts Formalismus - werden die Zahlen aus obiger Tabelle verwendet - sagt in diesem Fall, dass man eine Präferenz für das Kino besitzen darf, welche entweder die Stärke 3, 4 oder 5 aufweist. Der Akteur hat in diesem Fall eine Präferenz der Stärke 4, ins Kino zu gehen. Diese Präferenz beziehungsweise Wahl ist rational. Ebenso wäre es rational, wenn der Akteur eine Präferenz der Stärke von 3 oder 5 besitzen würde. Irrational wäre es beispielsweise, wenn der Akteur eine Präferenz der Stärke 6 besässe.

21 Man kann das Modell ordinal oder kardinal interpretieren (vgl. Kapitel 1 für eine Erläuterung, was die Begriffe ordinal und kardinal bedeuten). Ich habe bei Gert keine Aussagen gefunden, die einer ordinalen Deutung widersprechen. 
Gemäss den Werten des Akteurs wäre es nicht in diesem Masse wertvoll, ins Kino zu gehen.

Obiger Formalismus und die dahinterstehende Grundannahme wird von Gert nun wie folgt auf den Begriff der Vergleichbarkeit angewendet: Zwei Optionen, $A$ und $B$, sind genau dann traditionally comparable, wie Gert sagt, wenn die Ränge von $A$ und $B$ keine Überlappungen haben. $A$ ist genau dann besser als $B$, wenn der tiefste Wert von $A$ grösser als der grösste Wert von $B$ ist. ${ }^{22}$ Gert nennt dies die Range-Rule (nennen wir es Bereichsregel).

Ein Beispiel: $A(4,6) ; B(2,3)$. Der tiefste Wert entspricht der kleinsten rational zulässigen Präferenz für die Option. Der höchste Wert entspricht der höchsten rational zulässigen Präferenz für die Option. In diesem Fall sind die beiden Optionen traditionell vergleichbar (traditionally comparable). $A$ ist besser als $B$.

Dasjenige, was Gert traditionally comparable nennt, kann dabei so verstanden werden, dass zwischen $A$ und $B$ eine der drei Relationen der Trichotomie-These instanziiert ist. ${ }^{23}$

\section{Erklärung von Parität}

Gert modelliert Parität mithilfe seiner Theorie nun wie folgt: $A$ und $B$ sind genau dann on par, wenn $A$ und $B$ die gleichen Werte besitzen. Ein Beispiel: $A(1,2) ; B(1,2)$. Es impliziert, dass, wenn $A$ besser als $C$, dann auch $B$ besser als $C$ ist.

In diesem Punkt täuscht sich Gert allerdings. Er modelliert Changs Ansatz in seinem Modell falsch. Gerts Definition von Parität bildet Changs Idee nicht richtig ab. Das erwähnt auch Rabinowicz in seinem Aufsatz 'Modeling Parity and Incomparability'. Rabinowicz schreibt: 'Gert's own defintion of parity is much narrower.' als $B$, wenn der höchste Rang von $A$ kleiner als der kleinste Rang von $B$ ist.

23 Man könnte meinen, dass diese Gleichsetzung falsch ist. Deshalb, weil die Trichotomie-These zusätzlich die Relation der Indifferenz beinhaltet. Gert fängt dies in seinem Formalismus aber damit ein, dass $A$ und $B$ genau dann gleich gut sind, wenn ein Akteur für $A$ und $B$ die gleiche rational zulässige Präferenz besitzt. Dies gilt nach Gert per definitionem nicht als Überlappung. Bei einer Indifferenz ist man dementsprechend rational verpflichtet, indifferent zu sein. Falls $A$ und $B$ nur eine Präferenz-Stärke nicht gemeinsam haben, ist dies nicht erfüllt. 
(Rabinowicz 2004: 12). Rabinowicz hat Recht. Nehmen wir an, dass $A$ und $B$ im Sinne von Chang on a par sind. Was dies genau bedeutet, ist wie erwähnt unklar. Was sich aber eindeutig sagen lässt, ist, dass $A$ und $B$ gemäss Chang in diesem Fall in einem bestimmten intransitiven Verhältnis zueinanderstehen müssen. Nicht-Transitivität ist gemäss Chang charakteristisch für Parität. Die gleichen Ränge in Gerts Modell implizieren hingegen Transitivität. Genauer ausgedrückt schliessen die gleichen Ränge aus, dass $A+$ besser als $A$, nicht aber besser als $B$ ist. Im Argument der kleinen Verbesserung stellt dies jedoch eine zentrale Annahme dar (vgl. Kapitel 5). Falls man Parität so wie Gert definiert, ist das Argument der kleinen Verbesserung dementsprechend nicht anwendbar.

Es ist allerdings möglich, Changs Idee im Sinne von Gert in dessen Theorie abzubilden. Es reicht, dass es rational erlaubt ist, $A$ gegenüber $B$ zu präferieren und $B$ gegenüber $A$ zu präferieren. Meiner Ansicht nach müsste das Argument der kleinen Verbesserung in Gerts Modell zum Beispiel wie folgt wiedergegeben werden: $A(3,6) ; B(4,9) ; A+(7,8)$.

$A+$ ist besser als $A$, weil der tiefste Wert von $A+$ grösser als der höchste Wert von $A$ ist. $A$ und $B$ haben überlappende Werte und sind ergo nicht vergleichbar. $A+$ und $B$ haben ebenfalls überlappende Werte und sind ergo ebenfalls nicht durch die Trichotomie-These miteinander verbunden.

\section{Kritik}

Gerts Modell beinhaltet Schwachpunkte. Eine dieser Schwächen besteht darin, dass bestimmte Fälle in Gerts Modell nicht abgebildet werden können (vgl. Rabinowicz 2005 und Rabinowicz 2008).

$\mathrm{Zu}$ diesem Zweck können wir das Argument der kleinen Verbesserung aufgreifen und erweitern: $A+$ ist besser als $A$; beide sind weder besser noch schlechter als $B$. Das ist bekannt aus dem fünften Kapitel. Als neue Option wird nun $B+$ eingeführt. $B+$ ist besser als $B$. $B+$ ist aber nicht besser als $A$. $B+$ steht etwa für eine Musikerkarriere plus ein zusätzliches Monatsgehalt. Diese Konstellation kann in Gerts Modell nicht erfasst werden. Man betrachte zu diesem Zweck folgendes Argument: 
1. $A+$ ist besser als $A$ genau dann, wenn der tiefste Rang von $A+$ grösser als der höchste Rang von $A$ ist.

2. $B+$ ist besser als $B$ genau dann, wenn der tiefste Rang von $B+$ grösser als der höchste Rang von $B$ ist.

3. Der höchste Rang von $A$ ist grösser als/gleich gross wie der höchste Rang von $B$.

4. Der höchste Rang von $B$ ist grösser als/gleich gross wie der höchste Rang von $A$.

$\therefore$ Der tiefste Rang von $A+$ ist grösser als der höchste Rang von $B$ / ( $A+$ ist besser als $B$ ) aus (1) und (3).

$\therefore$ Der tiefste Rang von $B+$ ist grösser als der höchste Rang von $A$ / ( $B+$ ist besser als $A$ ) aus (2) und (4).

Wie obiges Argument zeigt, folgt in Gerts Modell, dass entweder $A+$ besser als $B$ oder $B+$ besser als $A$ ist. Genau dies dürfte jedoch nicht der Fall sein. Weder dürfte die marginal verbesserte Anwaltskarriere besser als die Musikerkarriere noch die marginal verbesserte Musikerkarriere besser als die Anwaltskarriere sein. ${ }^{24}$

Eher technische Schwierigkeiten dieser Art haben Rabinowicz dazu veranlasst, Gerts sogenanntes Intervall-Modell durch ein sogenanntes Intersection-Modell, wie es in der Ökonomie Anwendung findet, zu ersetzen (vgl. Rabinowicz 2008). Die entscheidende Differenz besteht darin, dass nicht mehr mit Intervallen, sondern mit Klassen von erlaubten Präferenzordnungen gearbeitet wird. Gemäss dem Modell von Rabinowicz kann obige Kombination von Präferenzen wie folgt erfasst werden:

$\begin{array}{ll}\text { Präferenzordnung 1: } & A+>A>B+>B \\ \text { Präferenzordnung 2: } & B+>B>A+>A\end{array}$

In diesem Fall besteht die Klasse aus zwei erlauben Präferenzordnungen.

Im nächsten Abschnitt wird uns nun die Frage interessieren, ob Gerts Deutung von Parität etwas dazu beitragen kann, in harten 
Fällen Vergleichbarkeit herzustellen. Falls ich es richtig sehe, macht es diesbezüglich keinen Unterschied, ob Gerts Modell oder, wie bei Rabinowicz, zusätzlich sogenannte Intersection-Operationen verwendet werden. Deshalb nicht, weil zwar der formale Apparat verändert wird, die Grundidee jedoch dieselbe bleibt. Nach Gert meint Parität, dass es erlaubt ist, sowohl die eine wie auch die andere Option zu bevorzugen. Im Modell von Rabinowicz besteht zwischen zwei Optionen, $A$ und $B$, genau dann die Relation der Parität, wenn in einer erlaubten Präferenzordnung $A$ gegenüber $B$ und einer anderen erlaubten Präferenzordnung $B$ gegenüber $A$ vorgezogen wird.

\subsection{Können harte Fälle mithilfe von Parität aufgelöst werden?}

Changs Ausführungen zu Parität helfen uns nicht weiter. Harte Fälle können durch die Aussage, dass es harte Fälle gibt, nicht aufgelöst werden. Das heisst nicht, dass uns Chang keinerlei Überlegungen präsentiert, wie man als Akteur mit einem harten Fall umgehen soll. Ihre Idee, welche im letzten Kapitel aufgegriffen wird, besteht darin, dass man sich via Bekenntnis mit einer Option mehr identifizieren kann. Es ist jedoch nicht nachvollziehbar, was dies mit einer zusätzlichen Vergleichsrelation zu tun haben soll. Vergleichsrelationen, so meine ich, haben einen bestimmten Zweck zu erfüllen. Sie sollen zwischen Optionen eine einfache Ordnung herstellen und den Übergang von den möglichen zu den gewählten Alternativen gewährleisten. Von einem rein formalen Standpunkt aus gesehen, gibt es keine Notwendigkeit, dass genau drei Vergleichsrelationen existieren. ${ }^{25}$ Aber pragmatisch betrachtet, gibt es einen guten Grund, von drei Vergleichsbeziehungen auszugehen - im Kontext einer einzelnen Entscheidung zwischen sich ausschliessenden Optionen. Gemäss einem pragmatischen Blickwinkel lassen sich Vergleichsrelationen nämlich grundsätzlich in einer Wahl ausdrücken, gegeben, dass gewisse Bedingungen wie Transitivität erfüllt sind (vgl. Kapitel 6). Man kann sagen, dass der Zweck von Vergleichsrelationen derjenige ist, dass 
sie handlungsleitend sein können. ${ }^{26}$ Wenn $x$ und $y$ hingegen unvergleichbar sind, dann ist es (i) unbestimmt, welche Option wertvoller ist und für welche Option die besseren Gründe sprechen, und (ii) unklar, wofür man sich entscheiden soll. Dabei ist wichtig zu sehen, dass es in dieser Kritik nicht darum geht, ob Chang denkt, ob Parität handlungsleitend ist oder nicht. Es geht darum, was plausibel ist. Und von einem pragmatischen Blickwinkel aus ist es plausibel davon auszugehen, dass Vergleichsrelationen handlungsleitend sein sollen.

Wie lautet die Antwort im Hinblick auf Gerts Modell? Können die Optionen in harten Fällen mithilfe von Gerts Interpretation von Parität verglichen werden? Dies ist meiner Meinung nach ebenfalls nicht möglich.

Zunächst kann man sich fragen, ob man nicht immer genau dann $x$ gegenüber $y$ präferiert, wenn man $x$ für wertvoller hält (Gert unterscheidet das in seinem Modell).

Das Hauptproblem von Gerts Ansatz ist jedoch das folgende: Harte Fälle werden nicht richtig dargestellt, wenn eine Präferenz mit einer Wahl gleichgesetzt wird. Eine Theorie aufgedeckter Präferenzen (revealed preference) kann in diesem Zusammenhang nicht überzeugen (vgl. Kapitel 4). Man kann Gert zustimmen, dass ein Akteur eine Präferenz in seinem Sinn haben respektive eine Wahl treffen kann (bei Gert ist dies bekanntlich dasselbe). Dies ist aber nicht hinreichend dafür, um die Optionen als vergleichbar auszuweisen. Dazu kommt, dass Gerts Deutung von Parität ebenfalls nicht handlungsleitend ist.

Man könnte einwenden, dass ein Akteur gemäss Gerts Interpretation von Parität, und genau gleich wie bei der Relation der Indifferenz, beide Optionen wählen kann. In Gerts Modell unterscheidet sich Parität wie folgt von Unvergleichbarkeit: Im Gegensatz zu Unvergleichbarkeit, welche durch eine Abwesenheit einer Präferenz wenden will, erschöpft sich Vergleichbarkeit pragmatisch gesehen in drei Vergleichsrelationen: Wenn $x$ mehr Wert als $y$ hat, dann haben wir am meisten Gründe, $x$ zu wählen und dann sollen wir $x$ wählen. Wenn $x$ weniger Wert als $y$ hat, dann haben wir die meisten Gründe, $y$ zu wählen und sollen y wählen. Wenn $x$ und $y$ gleich viel Wert haben, dann sind wir frei, entweder $x$ oder $y$ zu wählen. Diese Überlegung finden wir selbst in Chang 2013a: 17. 
charakterisiert werden kann, zeichnet sich Parität dadurch aus, dass es mehrere zulässige Präferenzen gibt. Gemäss Gerts Deutung von Parität darf eine Akteurin zum Beispiel sowohl eine Präferenz für den Beruf der Anwältin als auch eine Präferenz für den Beruf der Klarinettistin besitzen.

Das Problem bei dieser potenziellen Erwiderung liegt jedoch darin, dass ein Akteur im Falle von Unvergleichbarkeit überhaupt keine Präferenz oder kein Werturteil bilden kann.

Gerts Modell stellt ein normatives Konzept dar. Die Normativität kommt ins Spiel, wenn Präferenzen als zulässig oder nicht zulässig bewertet werden. Dies jedoch setzt voraus, dass ein Akteur Präferenzen und Werturteile über Präferenzen bilden kann. Aber genau dies ist im Falle von Unvergleichbarkeit nicht erfüllt. Gerts Modell zur rationalen Auflösung harter Fälle setzt etwas voraus, was in einem harten Fall nicht gegeben ist. Ergo ist das Modell nicht anwendbar.

\subsection{Zusammenfassung}

Als Erstes wurde Changs Deutung von Parität eingeführt. Ich habe argumentiert, dass es sich dabei um eine sprachliche Umformulierung der Aussage handelt, dass es harte Fälle gibt. Dann wurde Gerts Theorie von Parität dargelegt und auf Changs Idee angewendet. Drittens wurde die Frage, ob in harten Fällen mithilfe von Parität Vergleichbarkeit erzielt werden kann, negativ beantwortet.

Unabhängig davon, wie Parität genau definiert wird, sollte man bedenken, dass ein wichtiger Zweck von Vergleichsrelationen darin besteht, dass sie handlungsleitend sind. Eine Vergleichsrelation sollte im paarweisen Vergleich eine einfache Ordnung zwischen den Optionen herstellen können.

Neben dieser allgemeinen Überlegung besteht das spezifische Problem von Gerts Modell darin, dass es auf einer problematischen Gleichsetzung beruht: Wenn Präferenz synonym mit Wahl verstanden wird, stellt dies keine adäquate Charakterisierung eines harten Falles dar. Zudem wird in Gerts Modell vorausgesetzt, dass ein Akteur Werturteile über Präferenzen bilden kann. Aber genau dies ist im Falle von Unvergleichbarkeit nicht gegeben. 
Gerts und Changs Idee, dass man in harten Fällen beides wählen darf, scheint notabene nicht abwegig zu sein. Aber nicht nur braucht es dafür eine plausible Begründung, auch muss diese These stark präzisiert und eingeschränkt werden (sie gilt nämlich nur unter ganz bestimmten Bedingungen). Ich werde im letzten Kapitel versuchen, eine solche Begründung zu liefern und die Bedingungen herauszuarbeiten, unter welchen beide unvergleichbaren Optionen gewählt werden dürfen. Im nächsten Kapitel geht es nun aber zuerst um eine alternative Lösung, die Optionen in harten Fällen als vergleichbar auszuweisen. 



\section{Kapitel 9}

Klumpentheorie 
In den letzten Kapiteln wurde die Möglichkeit diskutiert und zurückgewiesen, dass die Optionen in harten Fällen deshalb vergleichbar sind, weil sich Vergleichbarkeit nicht in drei Vergleichsrelationen erschöpft.

In diesem Kapitel wird eine alternative Möglichkeit betrachtet, wie die Rationalität in einem harten Fall aufrechterhalten werden könnte. Es geht um die sogenannte 'Klumpentheorie', die auf Hsieh zurückgeht (Hsieh 2005). Die 'Klumpentheorie' ist eine Theorie über Deckwerte. Eine philosophische Theorie hat bekanntlich oftmals den Anspruch, etwas Grundlegendes auf den Punkt zu bringen und damit eine gewisse Allgemeingültigkeit zu besitzen. Bei der 'Klumpentheorie' verhält sich dies nicht anders. Dieser Ansatz weist in dem Sinne einen Allgemeingültigkeitsanspruch auf, als mit den Relata der Vergleichbarkeit - dasjenige, was verglichen wird - sehr unterschiedliche Dinge gemeint sein können: diverse Berufe, unterschiedliche Fussballer oder verschiedene philosophische Texte. Diese Handlungsoptionen, Personen oder Gegenstände werden in sogenannte Klumpen eingeteilt. ${ }^{27}$ Klumpen können als Klassifizierungen interpretiert werden, die bestimmen, ob eine Handlungsoption einen Wert, wie etwa ein guter Beruf zu sein, hinreichend erfüllt. Damit ist gemeint, dass die Einteilung in Klassen viele Unterschiede erlaubt und es ermöglicht, die betreffenden Handlungsoptionen dennoch als gegebenenfalls vergleichbar, beispielsweise als gleich gut, zu bewerten. ${ }^{28}$

Können die Optionen in harten Fälle mithilfe der 'Klumpentheorie' tatsächlich verglichen werden?

Hsieh spricht in diesem Zusammenhang nicht von 'Option', 'Gegenstand' oder 'Person', sondern benutzt den allgemeinen Platzhalter 'item'.

28 Die 'Klumpentheorie' kann als Reaktion auf Changs Idee der Parität sowie Griffins und Parfits Ansatz der groben oder ungefähren Gleichheit (rough equality) verstanden werden - beides mögliche alternative Formen der Vergleichbarkeit. Chang, Parfit und Griffin denken, dass die Trichotomie-These falsch oder zumindest problematisch ist. Hsieh hält an der Trichotomie-These fest, in dem Sinne, dass es keine alternativen Vergleichskonzepte braucht. Wenn man nämlich das Konzept der Vergleichbarkeit untersucht, so Hsieh, zeigt sich, dass viele unvergleichbar scheinende Dinge - nach Hsieh zum Beispiel Mozart und Michelangelo - in Wirklichkeit vergleichbar sind und durch die Trichotomie-These zusammenhängen. Dies soll aus der sogenannten 'Klumpigkeit' (clumpiness) der Deckwerte folgen. Parität beispielsweise ist nach Hsieh dasselbe wie Gleichheit. Mich interessiert aber nicht die Anwendung der 'Klumpentheorie' auf Konzepte wie Parität, sondern, ob damit in harten Fällen Vergleichbarkeit hergestellt werden kann. 
Um diese Frage beantworten zu können, wird in einem ersten Schritt die Theorie eingeführt und werden die wichtigsten Thesen dann erläutert. In einem zweiten Schritt wird die 'Klumpentheorie' diskutiert. Dabei werden Erkenntnisse und Schwächen darin aufgezeigt. Im dritten Schritt wird der Frage nachgegangen, ob die 'Klumpentheorie' in harten Fällen Vergleichbarkeit erzielen kann. Harte Fälle können gemäss diesem Ansatz unter Umständen dadurch aufgelöst werden, dass bei der Entscheidungsfindung ein Deckwert herbeigezogen werden kann, hinsichtlich dessen die relevanten Dinge gewichtet werden können. Daraus kann folgen, dass die Optionen trotzdem vergleichbar sind. Ich argumentiere, dass das Vorliegen eines Deckwerts tatsächlich eine begründete Entscheidung ermöglichen könnte. Das Problem ist jedoch, dass dies mit dem Konzept, das der 'Klumpentheorie' zugrunde liegt, nicht oder unter Umständen 'nur' in einigen sehr wenigen Fällen gelingt. Dabei zeige ich anhand eines Beispiels, (i) mit welchem Konzept eines Deckwerts man Unvergleichbarkeit auflösen könnte, (ii) weshalb uns genau diese Art von Deckwert nicht zur Verfügung steht, (iii) was Chang darauf entgegnen würde - Deckwerte sind sogenannte namenlose Werte - und (iv) weshalb Changs Ansatz nicht überzeugen kann.

\subsection{Die Theorie}

Die 'Klumpentheorie' ist in der Literatur bislang kaum diskutiert worden. ${ }^{29}$ In diesem Abschnitt geht es um die Grundlage dieser Versätze dar, die sich mit der Rationalität dieser Vergleichstheorie auseinandersetzen. Im Jahr 2007 ist ein Aufsatz erschienen, in dem die These vertreten wird, dass die 'Klumpentheorie' die Konsequenz hat, dass ein Akteur in sehr spezifischen Entscheidungssituationen, die in der entscheidungstheoretischen Literatur money pumps genannt werden - für eine kurze Erläuterung vgl. Kapitel 5 -, weniger Nutzen hat (Peterson 2007). Im Aufsatz von Peterson geht es darum, dass ein Akteur eine Sequenz von Entscheidungen trifft, und aufgrund der 'Klumpentheorie' folgt, dass er am Schluss schlechter gestellt ist als zu Beginn - man muss wohlgemerkt eine modifizierte Version von money pumps verwenden. Vereinfacht gesagt, würde dies bedeuten, dass die 'Klumpentheorie' nicht mit dem Konzept einer rationalen Wahl, so wie dieses Konzept in der entscheidungstheoretischen Literatur verwendet wird, in Einklang gebracht werden kann. Im Jahr 2012 ist aber eine Erwiderung veröffentlicht worden, in der dies bestritten wird (Steglich-Petersen 2012). Im Aufsatz 
gleichstheorie. Meiner Ansicht nach lässt sich Hsiehs Aufsatz 'Equality, clumpiness and incomparability' auf drei wesentliche Thesen reduzieren. Die 'Klumpentheorie' stellt einen Überbegriff für drei Ideen dar:

1. Wenn ein Deckwert existiert, bezüglich dessen man zwei Gegenstände oder Optionen vergleichen kann, hängen die zwei Gegenstände oder Optionen durch die Trichotomie-These zusammen. Dies bedeutet, dass in dem Sinne Vergleichbarkeit vorliegt, als ein Gegenstand besser oder schlechter als der andere Gegenstand ist oder beide Gegenstände gleich gut sind.

2. Inkommensurable Gegenstände - Gegenstände, die nicht hinsichtlich eines Deckwerts verglichen werden können - sind manchmal trotzdem vergleichbar. Dies ist genau dann der Fall, wenn der eine Gegenstand sämtliche für den Vergleich relevanten Qualitäten besser erfüllt.

3. Zwei Gegenstände sind genau dann unvergleichbar, wenn (i) kein Deckwert existiert, hinsichtlich dessen die beiden Gegenstände verglichen werden können, und (ii) keiner der beiden Gegenstände sämtliche für den Vergleich relevanten Qualitäten besser erfüllt.

Im Folgenden werden obige Thesen erläutert. Das Ziel in diesem Abschnitt besteht darin, Hsiehs Position adäquat wiederzugeben. $\mathrm{Zu}$ diesem Zweck werden auch eigene Beispiele zur Veranschaulichung herangezogen. Des Weiteren gehen einige Deutungen über das explizit Geschriebene bei Hsieh hinaus. Dies wird aber immer kenntlich gemacht. Es geht dementsprechend (noch) nicht darum, ob Hsiehs Position inhaltlich richtig ist.

von Steglich-Petersen wird erwidert, dass Petersons 'Geldpumpe' nicht funktioniert und ergo auch nicht folgt, dass die beiden Konzepte, das Rationalitätskonzept moderner Entscheidungstheorien und die 'Klumpentheorie', unvereinbar sind. Gemäss Steglich-Petersen verliert der Akteur in Petersons Beispiel Nutzen, weil sich die Umstände geändert haben. Dies, so Steglich-Petersen, dürfte bei einem money pump allerdings nicht geschehen. Man könnte einwenden, dass es bei dieser Frage - hat die 'Klumpentheorie' zur Konsequenz, durch modifizierte 'Geldpumpen' ausgebeutet zu werden? - um die Grundlage der Theorie geht. Schliesslich, so könnte man argumentieren, würde es dieses Vergleichskonzept unterminieren, wenn kein Link zum Konzept der Rationalität entscheidungstheoretischer Modelle möglich wäre. Dies mag sein. Dennoch, so meine ich, geht es dabei um eine weiterführende Frage. 
These 1 Wenn ein Deckwert existiert, bezüglich dessen man zwei Gegenstände vergleichen kann, hängen diese beiden Gegenstände durch die Trichotomie-These zusammen. Dies folgt aus der 'Klumpigkeit' der Deckwerte.

Was Hsieh mit dem terminus technicus der 'Klumpigkeit' meint, kann anhand eines Beispiels des Autors veranschaulicht werden:

Eine Dozentin vergleicht die Aufsätze zweier Studenten. Das Entscheidungskriterium respektive der Deck wert $(V)$, hinsichtlich dessen die Dozentin diese beiden Aufsätze vergleicht, lautet 'guter philosophischer Aufsatz' (Hsieh 2005: 185). Das Entscheidungskriterium setzt sich dabei aus verschiedenen Qualitäten zusammen. Ich werde diese der Einfachheit halber auf zwei Qualitäten, Q1 und Q2, reduzieren. Q1 kann beispielsweise für 'Originalität' und Q2 für 'Schreibstil' stehen.

Dass der Deckwert klumpig ist, bedeutet gemäss Hsieh Folgendes: Hinsichtlich des Deckwerts werden die beiden Aufsätze in Klassen (clumps) unterteilt. Je nachdem, wie gut ein Aufsatz die beiden Qualitäten erfüllt, kommt er in eine höhere oder tiefere Klasse. Falls die beiden Aufsätze in derselben Klasse sind, sind sie gleich gut. Dabei ist es denkbar, dass der eine Aufsatz beide Qualitäten besser erfüllt und sie sich trotzdem in derselben Klasse befinden beziehungsweise gleich gut sind. Dies ist deshalb der Fall, weil der Unterschied zwischen den beiden Aufsätzen für die Einordnung in die verschiedenen Klassen nicht relevant sein muss.

Der Begriff der Klumpigkeit meint also, dass wir die zu vergleichenden Gegenstände in Klassen einordnen, je nachdem, wie gut sie den für den Vergleich relevanten Deckwert erfüllen. Wenn man zwei Gegenstände vergleicht, vergleicht man gemäss der Klumpentheorie immer die Klassen, zu denen diese Gegenstände gehören. Der Deckwert 'guter philosophischer Aufsatz' lässt sich etwa in die Klassen oder Noten A, B , C ... unterteilen. Nehmen wir an, der eine Aufsatz wird mit A und der andere Aufsatz mit B bewertet. 'Aufsatz 1 ist besser als Aufsatz 2' bedeutet demnach, dass die Klasse, zu welcher der erste Aufsatz gehört, besser ist als die Klasse, zu welcher der zweite Aufsatz zählt. Aufsätze zu vergleichen ist nach Hsieh dasselbe wie Klassen zu vergleichen, zu denen diese Aufsätze gehören. Gemäss Hsieh werden 
die Unterschiede dabei ab einem bestimmen Punkt irrelevant für die Klasseneinteilung: '(...) clumps reflect the smallest units of measurement for purpose of comparison.' (Hsieh 2005: 198). Wie differenziert man die Klassen formulieren will, hängt vom beabsichtigten Zweck der Bewertung ab. So ist es denkbar, dass die Dozentin für die Bewertung einer Abschlussarbeit, im Gegensatz zu einer Bewertung eines kurzen Aufsatzes, eine sehr präzise Einteilung der Klassen benötigt. Gemäss diesem Ansatz können zwei Aufsätze dementsprechend bei einer Klasseneinteilung gleich gut und bei einer anderen, etwa einer feineren Klasseneinteilung, nicht gleich gut sein. ${ }^{30}$

Es ist wichtig zu sehen, dass Hsiehs Ansatz eine bestimmte Art der Vergleichbarkeit voraussetzt, welche er 'holistisch' nennt. Was dies bedeutet, kann anhand desselben Beispiels illustriert werden:

Einerseits vollzieht die Dozentin bei einem holistischen Vergleich eine sogenannte component comparison. Ich interpretiere dies so, dass die beiden Aufsätze sowohl hinsichtlich Schreibstil als auch hinsichtlich Originalität verglichen werden. Charakteristisch für eine component comparison ist meiner Meinung nach, dass man dabei mehrere Vergleiche tätigt. Ich stelle mir vor, dass der Vergleich des Gewichts zweier Menschen keine component comparison darstellt, weil dabei nicht mehrere Vergleiche vollzogen werden. Ich werde diesen technischen Begriff deshalb als ‘zusammengesetzter Vergleich' übersetzen.

Ein holistischer Vergleich enthält nach Hsieh aber noch eine zusätzliche Komponente, nämlich, dass die verschiedenen Qualitäten gewichtet werden. In obigem Beispiel sind dies Q1 und Q2. Das heisst, dass die Dozentin bestimmt, wie bedeutend Schreibstil und Originalität für den Deckwert 'guter philosophischer Aufsatz' sind. Man kann sich vorstellen, dass die Qualitäten der Originalität und des Schreibstils jeweils gleich wichtig sind. In diesem Fall werden sowohl Q1 als auch Q2 mit 0.5 gewichtet.

Zusammenfassend kann festgehalten werden, dass nach Hsieh die Möglichkeit, Gegenstände in Klassen einzuteilen, die Möglichkeit eines holistischen Vergleichs voraussetzt. Holistische Vergleiche ihrerseits setzen voraus, dass die zu vergleichenden Gegenstände

30 Andreou formuliert es wie folgt: 'In Hsieh's view, two options O1 and O2, and two resolutions R1 and R2, can be such that A is better than B when resolution R1 is in play and A is equal (not just roughly equal) to B when resolution R2 is in play.' (Andreou 2015: 15). 
hinsichtlich eines gemeinsamen Deckwerts verglichen werden können. Dies verhält sich gemäss Hsieh so, weil holistische Vergleiche im Gegensatz zu einem zusammengesetzten Vergleich den Aspekt der Gewichtung beinhalten. Wenn man beispielsweise sagt, dass der eine Aufsatz Q1 und der andere Aufsatz Q2 besser erfüllt, hat man einen zusammengesetzten Vergleich vorgenommen, wobei das Resultat des Vergleichs in diesem Fall unbestimmt ist. Das heisst, dass der eine Aufsatz zwar origineller, aber schlechter geschrieben und der andere Aufsatz gut geschrieben, aber wenig originell ist. Bei einem holistischen Vergleich werden diese beiden Qualitäten zusätzlich hinsichtlich eines Deckwerts gewichtet. Ein holistischer Vergleich, so kann man ergänzen, vereinigt die verschiedenen Qualitäten in einem einzigen Deckwert (Hsieh sagt dies nicht explizit).

An dieser Stelle muss darauf aufmerksam gemacht werden, dass Hsieh auf eine bestimmte Weise interpretiert wird: 'holistischer Vergleich' = 'zusammengesetzter Vergleich plus Gewichtung'. Diese Interpretation wird beispielsweise durch eine spezifische Textstelle nahegelegt, in welcher Hsieh zuerst erläutert, was er unter einem zusammengesetzten Vergleich versteht (man tätigt mehrere Vergleiche) und mit ' (...) however, a holistic comparison involves an additional step (...)' fortfährt, wobei mit diesem zusätzlichen Schritt der Aspekt der Gewichtung gemeint ist (Hsieh 2005: 185).

Ich denke aber, dass dies nicht die einzig zulässige Deutung darstellt. An einer anderen Stelle schreibt Hsieh, dass er selbst Körperlänge als klumpigen Wert verstanden wissen will. Damit ist gemeint, dass bestimmt werden muss, ob die Körperlänge etwa in Zentimetern oder Millimetern gemessen wird, und sich daraus unterschiedliche Klassen ergeben. Zwei Zwillinge, so Hsieh, können in Zentimetern gemessen gleich gross und in Millimetern gemessen nicht gleich gross sein.

Ich denke nicht, dass man einen zusammengesetzten Vergleich tätigt, wenn man die Körperlänge zweier Menschen vergleicht, da man dabei nicht mehrere Vergleiche tätigt, sondern allein auf die Grösse schaut. Falls also eine component comparison eine notwendige Bedingung für einen holistischen Vergleich darstellt, haben wir es in diesem Fall mit keiner holistischen Gegenüberstellung zu tun. Da die Möglichkeit, in Klassen einzuteilen, holistische Vergleiche voraussetzt, ist Körpergrösse kein klumpiger Wert. Körperlänge ist aber nach Hsieh 
ein klumpiger Deckwert. Das heisst, dass ein holistischer Vergleich im Gegensatz zur ersten Interpretation - nicht unbedingt aus einem zusammengesetztem Vergleich bestehen muss.

Unabhängig davon, welche Deutung wir wählen, gilt aber Folgendes: Werte lassen sich genau dann in Klassen einteilen, wenn die zu vergleichenden Gegenstände hinsichtlich eines gemeinsamen Deckwerts verglichen werden können. Lässt sich kein solcher Deckwert finden, können die Gegenstände auch nicht in Klassen eingeteilt werden.

Dies führt uns zur zweiten These der Klumpentheorie. Was passiert, wenn bei einem Vergleich kein Deckwert vorhanden ist?

Manchmal, so Hsieh, lässt sich nämlich kein solcher finden. In Hsiehs Terminologie ist dies gleichbedeutend damit, dass die Gegenstände inkommensurabel sind. ${ }^{31}$ Der Begriff der Inkommensurabilität bedeutet nach Hsieh, dass zwei Gegenstände nicht hinsichtlich eines Deckwerts verglichen werden können. Die zweite grundlegende These der Klumpentheorie kann demnach wie folgt dargestellt werden:

These 2 Inkommensurable Gegenstände können manchmal mittels eines zusammengesetzten Vergleichs verglichen werden.

Eine component comparison, also einen zusammengesetzten Vergleich, beschreibt Hsieh als 'specifying a set of values and making separate evaluations between the two items with respect to each value in the set' (Hsieh 2005: 182). Ich verstehe dies wie gesagt so, dass vom Akteur mehrere Vergleiche vollzogen werden. Falls sich nun kein Deckwert finden lässt und die zu vergleichenden Dinge inkommensurabel sind, besteht nach Hsieh immer noch die Möglichkeit, die Gegenstände mittels eines zusammengesetzten Vergleichs zu vergleichen: 'The account in this article, however, distinguishes a way in which items that are incommensurable can also be compared, but not in virtue of a common value.' (Hsieh 2005: 182). Ein solcher Vergleich ist also genau dann möglich, wenn der zusammengesetzte Vergleich zulässig, wenn inkommensurabel nach Hsieh das Gegenteil von kommensurabel bedeutet, wovon ich ausgehe. Was Hsieh explizit sagt, ist Folgendes: '(...) two items can be compared in terms of some value - by which is meant that they are commensurable (...)' (Hsieh 2005: 182). 
nicht unbestimmt ist. Damit ist gemeint, dass sich die verschiedenen Qualitäten nicht gegenseitig 'ausstechen'. Bei einem unbestimmten Vergleich ist beispielsweise der eine Aufsatz origineller und der andere besser geschrieben. Ein Vergleich ist hingegen genau dann bestimmt, um Hsiehs Terminologie zu gebrauchen, wenn der eine Gegenstand den anderen 'dominiert'. Dies bedeutet, dass dieser Gegenstand bezüglich keiner Qualität schlechter, bezüglich mindestens einer Qualität aber besser als der andere Gegenstand ist. Liegt eine solche Dominanz vor, dann ist ein Vergleich auch dann möglich, wenn kein Deckwert existiert.

Die zweite These kann wie folgt veranschaulicht werden: Man stelle sich vor, dass ein bestimmter Fussballer besser als ein anderer Fussballer ist, weil er jede relevante Qualität ('Spielübersicht', 'Torgefährlichkeit', 'Balltechnik' etc.) besser erfüllt. In diesem Fall sind die beiden Fussballer nach der Klumpentheorie vergleichbar, auch wenn kein Deckwert wie 'guter Fussballer' existiert, hinsichtlich dessen sich die beiden Spieler vergleichen lassen.

Man könnte einwenden, dass dies in dem Sinne unplausibel ist, als ein solcher Deckwert ja existiert. Aber dies muss Hsieh zufolge nicht unbedingt der Fall sein. Gemäss Hsieh existiert ein Deckwert nur dann, so scheint es zumindest, wenn verschiedene Qualitäten existieren, die hinsichtlich dieses Deckwerts gewichtet werden können. Ist keine solche Gewichtung möglich, existiert auch kein Deckwert.

Aus den ersten beiden Thesen ergibt sich somit eine dritte Idee:

These 3 Zwei Gegenstände sind genau dann unvergleichbar, wenn (i) kein gemeinsamer Deckwert existiert, hinsichtlich dessen die beiden Gegenstände verglichen werden können, und (ii) der zusammengesetzte Vergleich unbestimmt ist.

Müsste diese These in Form eines Slogans wiedergegeben werden, könnte dieser 'unvergleichbar, weil inkommensurabel' lauten. Unvergleichbarkeit kann im Rahmen der Klumpentheorie als Resultat von Inkommensurabilität verstanden werden. Dies kann so gedeutet werden, dass Inkommensurabilität die entscheidende Bedingung darstellt, unter der ein harter Fall eintritt (ich werde später in diesem Kapitel darauf zurückkommen). Differenzierter formuliert, lautet die 
dritte These aber, dass, wenn zwei Gegenstände inkommensurabel sind und der zusammengesetzte Vergleich unbestimmt ist, die zwei Gegenstände unvergleichbar sind.

Hsieh erwähnt in Anlehnung an Raz folgendes Beispiel, um dies zu veranschaulichen (Raz 1986: 332): Es werden drei Optionen verglichen: eine Karriere als Anwalt (A), eine Karriere als Anwalt plus tausend US-Dollar $(A+)$ und eine Karriere als Musiker $(B)$. In diesem Zusammenhang gilt, dass $A+$ besser als $A$, nicht aber besser als $B$ ist. Wir wissen aus Kapitel 5, dass dies nichts anderes als das Argument der kleinen Verbesserung darstellt. Dabei bezeichnet auch Hsieh dieses Argument beziehungsweise das Versagen von Transitivität als adäquate Strategie, um Inkommensurabilität festzustellen.

Ich habe in Kapitel 5 gezeigt, weshalb die Sachlage komplizierter ist. Abgesehen davon liefert Hsieh aber eine neue Deutung des Berufsbeispiels: Die verbesserte Anwaltskarriere ist hinsichtlich einer Qualität (des Lohns) besser als die ursprüngliche Anwaltskarriere. Daraus folgt aber nicht, dass ein gemeinsamer Deckwert wie 'guter Beruf' existiert, hinsichtlich dessen man die beiden Berufe vergleichen kann. Das heisst, dass zwischen den Berufen nur ein zusammengesetzter Vergleich möglich ist. Und gegeben, dass der zusammengesetzte Vergleich unbestimmt ist, sind die beiden Berufe unvergleichbar.

Damit sind die drei wichtigsten Thesen zusammengefasst, expliziert und interpretiert worden. Im nächsten Abschnitt geht es nun darum, relevante Erkenntnisse und Schwächen dieser Vergleichstheorie darzustellen.

\subsection{Diskussion}

In diesem Teil werden auch interpretatorische Überlegungen angestellt. Das Ziel ist aber systematischer Natur. Während der Gegenstand des ersten Teils eine adäquate Darstellung von Hsiehs Position war, geht es nun darum, ob sie inhaltlich überzeugend ist.

Die Erkenntnis besteht darin, dass die Klumpentheorie eine Erklärung liefert, weshalb Optionen manchmal unvergleichbar sein können. Diese besteht darin, dass Werte manchmal in dem Sinne inkommensurabel sind, als die Optionen nicht hinsichtlich eines 
gemeinsamen Deckwerts verglichen werden können. Dies kann bisweilen dazu führen, dass Optionen weder als besser oder schlechter als noch als gleich gut wie die jeweilig andere Option bewertet werden können.

Die Schwächen der Klumpentheorie werden in zwei Bereiche eingeteilt, unbeantwortete Fragen und Begriff:

1. Es bleibt unbeantwortet, wann bei einem Vergleich ein Deckwert vorliegt und wann nicht, was zur Folge hat, dass auch die Frage, wann zwei Gegenstände durch die Trichotomie-These zusammenhängen, offen bleibt. Ebenso ist die Charakterisierung des Deckwerts unzureichend.

2. Die Anforderungen, welche die Klumpentheorie an das Konzept der Vergleichbarkeit stellt, sind zu hoch. Zwei Gegenstände sind manchmal vergleichbar, wenn sie gemäss der Klumpentheorie unvergleichbar sein sollten. Daraus folgt, dass dieses Konzept nicht geeignet ist, um in einem harten Fall eine potenzielle Vergleichbarkeit der Optionen herzustellen. Durch eine leichte Modifizierung kann diese Schwierigkeit allerdings behoben werden.

Beginnen wir mit dem Erkennen und der Erklärung von Unvergleichbarkeit.

\section{Erklärung von Unvergleichbarkeit}

Hsieh liefert eine Erklärung, weshalb Optionen unvergleichbar sein können. Der Grund dafür ist, dass Optionen manchmal inkommensurabel sind und der zusammengesetzte Vergleich (die component comparison) unbestimmt ist. Anders formuliert heisst dies, dass kein Entscheidungskriterium vorliegt und die für den Vergleich relevanten Komponenten nicht systematisch erfasst werden können. Man kann dies so interpretieren, dass daraus folgen kann, dass die Optionen unvergleichbar sind. ${ }^{32}$ 
Was dies konkret bedeutet, kann anhand des Fussballerbeispiels veranschaulicht werden: Die FIFA will den besten Fussballer der letzten hundert Jahre bestimmen. Weil Lionel Messi mit Argentinien noch nicht Weltmeister geworden ist, will die FIFA wissen, ob Maradona oder Pelé der bessere Fussballer war. Nehmen wir an, dass sich das zuständige Gremium darauf einigt, dass kein abstraktes Entscheidungskriterium wie 'guter Fussballer' formuliert werden kann. Dies heisst nichts anderes - habe ich die erste These der Klumpentheorie richtig interpretiert -, als dass keine Gewichtung der einzelnen Qualitäten möglich ist. Nehmen wir an, dass die FIFA für den Vergleich folgende Merkmale als relevant ansieht: 'Gewonnene Titel'; 'Geschossene Tore'; 'Allgemeines Ballgefühl', 'Technik unter Druck', 'Zweikampfstärke', 'Spielintelligenz', 'Antizipationsvermögen', 'Schnelligkeit', 'Torgefährlichkeit', 'Kreativität' und 'Spielübersicht'. Und nehmen wir weiter an, dass Maradona auf dem Fussballplatz torgefährlicher und zweikampfstärker, aber weniger kreativ und technisch schlechter als Pelé war. Es kann folgen, dass die Optionen 'Pelé erhält den Preis für den besten Fussballer' und 'Maradona erhält den Preis für den besten Fussballer' unvergleichbar sind.

Hsieh würde wohl sagen, dass 'Es gibt kein Entscheidungskriterium, und Pelé und Maradona erfüllen die relevanten Qualitäten unterschiedlich gut' bereits Unvergleichbarkeit ist, während ich sage, dass Unvergleichbarkeit daraus folgen kann. Hsieh benutzt zwar den Begriff der Inkommensurabilität, nicht aber denjenigen der Unvergleichbarkeit auf gleiche Weise, wie die beiden Termini in der vorliegenden Arbeit verwendet werden. Gemäss dieser Arbeit liegt genau dann Unvergleichbarkeit vor, wenn eine Akteurin keine begründete Präferenz bilden kann.

Meine Auffassung von Unvergleichbarkeit scheint allerdings kompatibel mit Hsiehs Position zu sein. Man kann sagen, dass die FIFA-Mitglieder keine Präferenz konstruieren können. Hsieh schreibt nämlich auch: 'The account that I advance can be understood as interpreting incomparability as the incompleteness of preferences.' (Hsieh 2005: 183). Dies kann wie folgt verstanden werden: Unvergleichbarkeit ist nach Hsieh dasselbe wie der Fakt, dass kein Deckwert vorliegt und der zusammengesetzte Vergleich unbestimmt ist. In obigem Beispiel ist dies der Fall. Es liegt kein Deckwert wie 
'guter Fussballer' vor, und der zusammengesetzte Vergleich ist unbestimmt. Maradona ist torgefährlicher, aber weniger kreativ als Pelé. Daraus kann folgen, dass die FIFA-Mitglieder keine Präferenz bilden können. Sie können nicht sagen, ob Maradona oder Pelé den Preis erhalten soll. Dies wiederum kann so interpretiert werden, dass Inkommensurabilität eine Bedingung darstellt, unter der ein Akteur keine Präferenz formulieren kann.

Das Problem der Unvergleichbarkeit muss der Klumpentheorie zufolge dementsprechend so gedeutet werden, dass es ein Problem von Deckwerten ist. Genauer, so könnte man ergänzen, stellt es ein Problem abwesender Deckwerte dar. Nicht immer, wenn ein solcher Deckwert benötigt wird, ist auch einer vorhanden. ${ }^{33}$

\section{Unbeantwortete Fragen}

Die Klumpentheorie lässt zwei wichtige Fragen offen:

1. Wann ist ein Deckwert vorhanden?

2. Was muss darunter präzise verstanden werden?

Dass die erste Frage unbeantwortet bleibt, und damit werde ich beginnen, ist aus folgendem Grund problematisch: Die Klumpentheorie kann als spezifisches Vorgehen betrachtet werden, ob und unter welchen Umständen zwei Gegenstände vergleichbar sind. Es besteht aus zwei Schritten:

Schritt 1: Liegt ein Deckwert vor? Falls ja, sind die Gegenstände vergleichbar. Falls nein, ist noch offen, ob die Gegenstände vergleichbar sind.

Schritt 2: Erfüllt ein Gegenstand sämtliche für den Vergleich relevanten Kriterien besser? Falls ja, sind die Gegenstände vergleichbar (auch wenn kein Deckwert vorhanden ist). Falls nein, sind die Gegenstände unvergleichbar.

33 Dies stellt eine alternative Erklärung von Unvergleichbarkeit dar, wobei argumentiert werden kann, dass auch in diesem Fall Wertkonflikte das entscheidende Argument bilden und die in Konflikt stehenden Werte in den unterschiedlichen Qualitäten zum Ausdruck kommen. Wenn der Deckwert 'guter Beruf' lautet, stellen die Werte 'Spass' und 'gesellschaftlicher Einfluss’ die Qualitäten dar. 
Bezüglich des ersten Schritts liefert uns Hsieh jedoch keine Antwort. Er ist, etwa im Gegensatz zu Chang, der Meinung, dass beim Vergleichen nicht immer ein Deckwert vorliegt. Das Vorhandensein oder die Abwesenheit eines Deckwerts stellt nach Hsieh die entscheidende Differenz zwischen Unvergleichbarkeit und Gleichheit dar. Dies hält auch Pinkowski in seiner kurzen Darstellung der Klumpentheorie fest: 'Why is it sometimes the case that options are deemed equal (of the same clump), but other times they should be considered incomparable? Hsieh argues that the problem is one of covering values.' (Pinkowski 2013: 464) Warum aber, dazu sagen weder Hsieh noch Pinkowski etwas, lässt sich beim Vergleichen von Gegenständen manchmal ein solcher Deckwert finden und manchmal nicht? Warum lassen sich nach Hsieh beispielsweise philosophische Aufsätze oder Mozart und Michelangelo hinsichtlich eines gemeinsamen Deckwerts vergleichen? Und weshalb können der Beruf der Musikerin und der Beruf der Anwältin nicht hinsichtlich eines gemeinsamen Deckwerts verglichen werden? Hsieh schreibt nämlich: 'In the case of the career choice example, like Raz, I take it that the legal career and the musical career are not equally good, because they are incomparable.' (Hsieh 2005: 200). Anders formuliert: Weshalb gibt es ein Entscheidungskriterium 'guter philosophischer Aufsatz', aber kein Entscheidungskriterium 'guter Beruf'? Wir wissen, dass sich Deckwerte nach Hsieh nur dann in Klassen einteilen lassen, wenn die zu vergleichenden Gegenstände hinsichtlich eines gemeinsamen Deckwerts verglichen werden können. Wenn unbestimmt bleibt, wann ein Deckwert vorliegt, bleibt auch unbestimmt, wann Werte klumpig sind und wann wir es mit einem zusammengesetzten Vergleich (ohne Möglichkeit zu gewichten) zu tun haben. Ergo bleibt auch unbestimmt, ob die Gegenstände durch die Trichotomie-These zusammenhängen oder nicht. Und folglich wird eben auch nicht beantwortet, ob die Gegenstände vergleichbar sind. Die Klumpentheorie kann nicht angeben, unter welchen Bedingungen Gegenstände vergleichbar sind.

Die zweite offene Frage ist, wie das Konzept des Deckwerts interpretiert werden muss. Insbesondere ist unklar, ob die Idee darin besteht, dass ein Deckwert mehr als die Summe seiner Komponenten darstellt. Besteht das Entscheidungskriterium 'guter Fussballer' aus den Kriterien 'Allgemeines Ballgefühl', 'Technik unter Druck', 'Zwei- 
kampfstärke' ... oder aus mehr als der Summe dieser Qualitäten? Ich habe Hsieh so interpretiert, dass ein Deckwert dasselbe wie die Summe der Kriterien plus die Gewichtung dieser Kriterien darstellt. Wenn also die relevanten Kriterien bekannt sind - wie diese ausgewählt werden, sagt die Klumpentheorie nicht -, kommt beim Deckwert inhaltlich nichts dazu. Die Qualitäten werden lediglich noch gewichtet. Ein Deckwert stellt somit nichts Weiteres als die Gewichtung der Kriterien dar.

Weshalb stellt es ein Problem dar, dass die zweite Frage nicht beantwortet wird? Es ist vor allem hinsichtlich der ersten unbeantworteten Frage von Bedeutung. Es bedeutet für den ersten offenen Punkt Folgendes: Die Klumpentheorie beantwortet die Frage 'Wann liegt ein Deckwert vor?' insofern nicht, als sie nicht angibt, wann sich die Kriterien gewichten lassen. Weshalb können die Kriterien im Falle der philosophischen Aufsätze gewichtet und im Falle unterschiedlicher Berufe nicht gewichtet werden? Weshalb können 'Schreibstil' und 'Originalität' gewichtet und 'Spass' und 'gesellschaftlicher Einfluss' nicht?

Es stellt sich aber ohnehin die Frage, wie zentral der Aspekt der Gewichtung ist, um Vergleichbarkeit gewährleisten zu können. Ich habe in Kapitel 7 - genauer als Hsieh dies tut - gezeigt, welche Gewichtung vage Entscheidungskriterien zulassen. Aber in vielen Fällen im Alltag brauchen wir gar keinen Deckwert, um die Optionen vergleichen zu können. Darum geht es im nächsten Abschnitt. Die Frage, welche Konsequenz die Nicht-Beantwortung der ersten Frage für die Auflösung von harten Fällen hat, wird im letzten Teil des Kapitels wieder aufgenommen.

\section{$\mathrm{Zu}$ hohe Anforderung an Vergleichbarkeit}

Die Anforderungen, die gemäss Hsieh erfüllt sein müssen, damit zwei Gegenstände vergleichbar sind, sind zu hoch. Damit meine ich, dass Gegenstände oder Optionen manchmal vergleichbar sind, auch wenn sie gemäss Hsieh unvergleichbar sein sollten.

Es lässt sich folgendes Gegenbeispiel formulieren: Nehmen wir an, dass sich das Entscheidungskriterium 'guter Fussballer' aus den Kriterien 'Allgemeines Ballgefühl', 'Technik unter Druck' und 'Zwei- 
kampfstärke' zusammensetzt, aber in dem Sinn nicht vorhanden ist, als sich die unterschiedlichen Kriterien nicht gewichten lassen. Nehmen wir weiter an, dass der zusammengesetzte Vergleich unbestimmt ist. Das heisst zum Beispiel, dass Pelé mehr Ballgefühl, aber weniger Zweikampfstärke als Maradona besitzt. Gemäss Hsieh ist dies gleichbedeutend damit, dass Pelé und Maradona unvergleichbar sind.

Weshalb ist das unplausibel? Nehmen wir an, dass folgende Bedingung erfüllt ist: $\mathrm{Q} 1>\mathrm{Q} 2>\mathrm{Q} 3$. Diese Bedingung besagt, wie wichtig die für den Vergleich relevanten Qualitäten sind. Man kann sich vorstellen, dass 'Allgemeines Ballgefühl' wichtiger als 'Zweikampfstärke' und diese wiederum wichtiger als 'Technik unter Druck' ist.

In diesem Fall ist es plausibel, dass Pelé und Maradona vergleichbar sind. Deswegen, weil Pelé mehr allgemeines Ballgefühl als Maradona besitzt und dies zentraler als Zweikampfstärke ist.

Allgemeiner formuliert, lässt sich sagen, dass die Gewichtung eine zu hohe Anforderung darstellt. Zumindest dann, wenn damit eine genaue Quantifizierung gemeint ist. Diese Interpretation ist naheliegend. Das Fussballerbeispiel stammt zwar nicht von Hsieh selbst. Ich habe es eingeführt. Hsieh verwendet in diesem Zusammenhang das philosophische Aufsatzexempel. Die Beispiele sind in den wesentlichen Punkten aber völlig analog: Es gibt verschiedene Qualitäten wie 'Schreibstil' oder 'Zweikampfstärke' und potenzielle Deckwerte wie 'guter Fussballer' oder 'guter philosophischer Text'. Im Zusammenhang mit dem Aufsatz-Beispiel wählt Hsieh dabei Formulierungen wie ' (...) the specification of the requisite degree (...)' oder '(...) attaches a weight to each of the individual respects on the basis of their relative importance in determining the overall comparison (...)' (Hsieh 2005: 185). Es sind Formulierungen dieser Art, welche obige Leseart nahelegen.

Quantifizierbarkeit ist allerdings unnötig, um Vergleichbarkeit gewährleisten zu können. Wie obiges Beispiel zeigt, reicht eine ordinale Rangordnung der verschiedenen Qualitäten aus, um beide Fussballer als vergleichbar auszuweisen (dasselbe gilt auch für das Aufsatzbeispiel).

Dies kann innerhalb von Hsiehs Theorie aber ganz einfach dadurch korrigiert werden, dass die genaue Gewichtung durch eine ordinale Rangordnung ersetzt wird. 
Man könnte als Verteidigung von Hsieh unter Umständen einwenden, dass der Begriff der Unvergleichbarkeit ein technisches Konzept darstellt und im obigen Gegenbeispiel von einem anderen Begriff ausgegangen wird. Ich habe im ersten Kapitel selbst dafür plädiert, dass die Begriffe der Unvergleichbarkeit und der Inkommensurabilität keine normalsprachlichen Ausdrücke darstellen. Das Gegenbeispiel muss aber nicht so verstanden werden, dass Hsieh per se einen falschen Begriff von Unvergleichbarkeit verwendet. Es muss so interpretiert werden, dass es hinsichtlich der Möglichkeit, rationale Entscheidungen zu treffen, ein falscher Begriff ist. Die Anforderungen sind in dem Sinne zu hoch, als eine rationale Entscheidung keine Quantifizierung voraussetzt.

\subsection{Können harte Fälle mit der Klumpentheorie aufgelöst werden?}

Der Klumpentheorie zufolge besteht das Problem von Unvergleichbarkeit darin, dass es ein Problem abwesender Deckwerte ist. Dies stellt wohlgemerkt eine Erklärung und keine Auflösung von Unvergleichbarkeit dar. Die Theorie hat aber auch eine positive Kehrseite: Lässt sich ein Deckwert finden, dann sind die Optionen vergleichbar. Es ist dieser Gedanke, der für die rationale Auflösung von harten Fällen von Interesse sein könnte. Im Folgenden soll diese Möglichkeit diskutiert werden. ${ }^{34}$

In einem ersten Schritt belege ich, dass harte Fälle mit der Klumpentheorie nicht aufgelöst werden können. In einem zweiten Schritt zeige ich, mit welcher Interpretation eines Deckwerts in einem harten Fall Vergleichbarkeit erzielt werden könnte.

Betrachten wir folgendes Beispiel von Unvergleichbarkeit: Ein Gymnasiallehrer kündigt für die kommende Woche die letzte Englischprüfung vor dem Matura-Examen an. Ein Schüler, nennen wir ihn Simon, fragt den Lehrer, ob er die Prüfung nicht bereits diese

34 Die andere Möglichkeit, wie ein harter Fall gemäss der Klumpentheorie aufgelöst werden könnte, bestünde darin, dass eine Dominanz vorliegt. Damit ist gemeint, dass eine Alternative sämtliche relevanten Qualitäten besser erfüllt. Dies ist in einem harten Fall offenkundig nicht der Fall. 
Woche schreiben könne. Denn, so Simon, er habe die einmalige Gelegenheit, am Wochenende mit seiner Band in London ein Konzert zu spielen. Er komme am Sonntag spät nach Hause und werde dementsprechend am Montag nicht in der Verfassung sein, um eine Prüfung ablegen zu können. Der Lehrer entgegnet Simon, dass er keine Zeit habe, eine zusätzliche Prüfung zu schreiben. Daraufhin schlägt ihm Simon vor zu versprechen, niemandem in der Klasse etwas über den Inhalt des Tests zu verraten. Der Lehrer vertraut Simon und willigt ein. Einige Schüler, die dies beiläufig mitbekommen haben, schlagen Simon vor, dass er nur Anna und niemandem sonst den Prüfungsinhalt verraten solle. Denn, wenn Anna im Englischtest keine genügende Note schreibe, wird sie mit sehr grosser Wahrscheinlichkeit durch die Matura fallen. Simon sieht sich mit einem harten Fall konfrontiert: Einerseits ist es ihm wichtig, dass Anna, die er mag, die Matura besteht. Andererseits ist es ihm ebenso wichtig, dass er den Lehrer, den er ebenfalls mag, nicht enttäuscht und sein Versprechen ihm gegenüber einhält - was dabei wichtiger ist, weiss Simon jedoch nicht. Kann dieser harte Fall durch die Klumpentheorie aufgelöst werden? Zu diesem Zweck muss man sich zuerst fragen, ob ein Deckwert vorliegt. Diese Frage wird im Rahmen der Klumpentheorie nicht beantwortet.

Dementsprechend kann nicht gesagt werden, ob die Optionen im obigen Beispiel vergleichbar sind. Man könnte den Standpunkt einnehmen, dass Hsieh diesen Anspruch nicht stellt und diese Vergleichstheorie als konditionale These zu verstehen ist. Demzufolge sagt die Klumpentheorie 'nur', dass wenn ein Deckwert vorhanden ist, dann die Optionen vergleichbar sind. Was würde es der Klumpentheorie zufolge bedeuten, dass in obigem Beispiel ein Deckwert vorliegt? Es würde heissen, dass ein Deckwert existiert, hinsichtlich dessen 'Versprechen halten' und 'Anna besteht die Matura' gewichtet werden können. Falls damit eine genaue Quantifizierung gemeint ist, kann dies nicht gelingen. Die Gewichtung kann aber durch eine ordinale Rangordnung ersetzt werden. Könnte der harte Fall auf diese Weise mithilfe der Klumpentheorie gelöst werden? Ja, falls Simon einen allgemeinen Deckwert herbeiziehen könnte, hinsichtlich dessen sich die relevanten Qualitäten in eine ordinale Ordnung bringen lassen, liesse sich der harte Fall aufösen. Der springende Punkt von Unvergleichbarkeit besteht aber genau darin, dass die relevanten Werte nicht 
gegeneinander abgewogen werden können. In einem harten Fall kann nicht auf der Basis von den besten Gründen entschieden werden. Eine Akteurin kann wegen der evaluativen Unwissenheit keine Präferenz bilden. In der Konsequenz bedeutet dies, dass sich harte Fälle mithilfe dieser Vergleichstheorie nicht auflösen lassen.

Ist diese Schlussfolgerung unter Umständen zu voreilig? Was könnte im Sinne von Hsieh entgegnet werden?

(1) Es könnte eingewendet werden, dass im Beispiel ein Deckwert existiert, hinsichtlich dessen die Qualitäten gegeneinander abgewogen werden können - ich nehme der Theorie willen an, dass sich Dinge wie ‘ein Versprechen halten' als ‘Qualitäten’ bezeichnen lassen.

(2) Es könnte erwidert werden, dass obiges Beisiel wie das Berufsexempel einen Fall von Unvergleichbarkeit darstellt und die Klumpentheorie solche Fälle zulässt.

(3) Es könnte dafür plädiert werden, dass mithilfe dieser Vergleichstheorie in anderen Beispielen Vergleichbarkeit hergestellt werden kann.

(4) Es könnte eingewendet werden, dass die Klumpentheorie eine Vergleichstheorie darstellt und es im obigen Beispiel um Entscheidungen geht.

Zu den potenziellen Einwänden (2) und (3): Der harte Fall im Beispiel kommt durch einen Wertkonflikt zustande. Dieses Beispiel steht stellvertretend für sämtliche harten Fälle. Falls mithilfe der Klumpentheorie im Beispiel keine Vergleichbarkeit erzielt werden kann, sollte dies auch in sämtlichen anderen harten Fällen misslingen. Es ist denkbar, dass es Fälle von Unvergleichbarkeit gibt, welche nichts mit Wertkonflikten zu tun haben. Damit ist gemeint, dass es Fälle geben kann, in denen die Optionen weder besser noch schlechter als noch gleich gut wie die jeweilig andere Option sind, und alternative Begründungen existieren, weshalb sich dies so verhält. Ich schliesse weder aus, dass es solche Fälle gibt, noch, dass die Klumpentheorie in diesen Fällen Vergleichbarkeit erzielen kann. Für die vorliegende Arbeit ist das deshalb nicht relevant, weil es um andere Kasus geht (vgl. Kapitel 2 und 3). 
Zum potenziellen Einwand (4): Hsieh lässt bewusst offen, ob die Klumpentheorie in einem Entscheidungskontext anwendbar ist: $\mathrm{Er}$ schreibt: 'I understand comparison to be distinct from choice, and I take it to be an area of further inquiry as to how comparative relations of the sort discussed in this article function in the context of choice.' (Hsieh 2005: 199). Viele von Hsiehs Beispielen sind aber so gewählt, dass es sich um reale oder hypothetische Entscheidungssituationen handelt: Eine Dozentin muss sich für eine Note, ein Akteur für einen bestimmten Beruf entscheiden. Aber nehmen wir an, dass die Wahl der Beispiele missverständlich gewählt und die Klumpentheorie nicht auf einen Entscheidungskontext anwendbar ist. In diesem Fall wäre die Theorie ohnehin nicht geeignet, um in einem harten Fall Vergleichbarkeit herstellen zu können, da es sich bei harten Fällen um schwierige Entscheidungssituationen handelt.

Zum potenziellen Einwand (1): Existiert im Beispiel tatsächlich kein Deckwert? Ist es nicht denkbar, dass es einen grundlegenden Wert gibt, hinsichtlich dessen die unterschiedlichen Qualitäten gegeneinander abgewogen werden können? Wir wissen nicht, was Hsieh unter einem Deckwert genau versteht. Ist meine Interpretation richtig, dann meint ein Deckwert nichts anderes als die Gewichtung der unterschiedlichen Qualitäten. In diesem Fall fällt die Antwort negativ aus: Simon kann die relevanten Qualitäten - 'Versprechen halten' versus 'Anna besteht die Matura' - nicht einmal in eine ordinale Reihenfolge bringen, geschweige denn gewichten (im Sinne einer Quantifizierung).

Ein Deckwert kann allerdings auch anders interpretiert werden. Diese Deutung geht über Hsiehs Darstellung hinaus. Nämlich so, dass es etwas gibt, das wichtiger ist als das Versprechen gegenüber dem Lehrer und wichtiger als Annas Bestehen der Matura. So philosophisch abstrakt die Rede von Deckwerten klingen mag, die Idee, bei schwierigen Entscheidungen nach etwas Grundlegendem zu suchen, hinsichtlich dessen die relevanten Überlegungen gegeneinander abgewogen werden können, ist nicht abwegig. Falls es so etwas gibt und die beiden Dinge zu diesem übergeordneten Wert unterschiedlich beitragen, könnte die Unvergleichbarkeit mithilfe eines Deckwerts aufgelöst werden. Die Unmöglichkeit, die beiden Dinge ordinal zu ordnen, wäre dementsprechend nur scheinbar. Die Überlegungen im 
dritten Kapitel legen allerdings den Schluss nahe, dass uns in harten Fällen keine solchen allgemeinen Werte zur Verfügung stehen, welche die evaluative Unwissenheit auflösen könnten.

Chang würde widersprechen. Gemäss Chang können wir stets auf allgemeine Werte zurückgreifen. Die Position, die Chang vertritt, besteht darin, dass es sogenannte namenlose Deckwerte sind. Im Sammelband Incommensurability, Incomparability and Practical Reason geht sie davon aus, dass in jeder Entscheidungssituation ein Deckwert existiert: 'Every choice situation is governed by some value. Call this the choice value. The choice value is, roughly "what matters" in the choice situation.' (Chang 1997: 7) In einem Aufsatz mit dem Titel 'All-things-considered' formuliert sie es etwas vorsichtiger. Darin geht Chang davon aus, dass uns bei einem Vergleich meistens ein namenloser Deckwert zur Verfügung steht. Sie schreibt: '(...) I believe that, in many cases in which the considerations relevant to the all-things-considered judgement are very different, the more comprehensive value that accounts for their normative relations has no name.' (Chang 2004: 3). Dieser Wert dient dem Zweck, dass ein Akteur ein Urteil fällen kann, welche Option alles in allem besser ist. Dies wiederum kann als gleichbedeutend damit verstanden werden, dass der namenlose Wert bestimmt, wie wichtig die einzelnen Werte sind. Er determiniert, wie wichtig das Versprechen und Annas Bestehen der Matura sind.

Die Motivation, weshalb Chang namenlose Werte einführt, ist nachvollziehbar. Einzelne Werte oder Qualitäten, wie Hsieh sagen würde, können nach Chang nicht selbst determinieren, wie wichtig sie sind. Oder mit Chang ausgedrückt: '(...) the values at stake cannot determine their own normative relations in a given set of circumstances. Something with further content - normative content - is needed to do this normative work.' (Chang 2004: 13). Chang würde sagen, dass die einzelnen Qualitäten in Hsiehs Aufsatzbeispiel - 'Originalität', 'Schreibstil' ... - nicht selbst bestimmen können, wie wichtig sie sind, und es dafür einen Deckwert wie 'guter philosophischer Aufsatz' braucht, der die unterschiedlichen Qualitäten zusammenbringt. Changs Idee besteht nun darin, dass ein solcher Deckwert zwar nicht immer benannt werden kann, aber dennoch existiert. Er bestimmt dabei, wie die einzelnen Qualitäten normativ zusammenhängen. Mit normativ, verstehe ich es richtig, meint sie Folgendes: Einerseits 
können Argumente dafür genannt werden, weshalb einzelne Qualitäten relevant sind und andere nicht. Andererseits können Argumente dafür erwähnt werden, wie wichtig die für den Vergleich relevanten Qualitäten sind.

Changs Beweggrund, weshalb sie namenlose Deckwerte einführt, ist also wie erwähnt nachvollziehbar. In diesem Zusammenhang scheint aber eher der Wunsch Vater des Gedankens gewesen zu sein. Es kann nicht darum gehen, ob es nice to have wäre, dass solche Deckwerte existieren. Es sollte darum gehen, ob uns plausiblerweise namenlose Deckwerte zur Verfügung stehen.

Es ist nicht in erster Linie der Umstand, dass diese Werte namenlos sein sollen, der diese Position unplausibel macht. Der angebliche Fakt, dass namenlose Werte existieren, erscheint etwas kurios. Man könnte dies aber zugestehen. Chang verweist in diesem Zusammenhang auf Aristoteles. Gemäss Aristoteles existieren viele Werte, die keinen Namen haben. Damit spielt Chang auf Aristoteles' Tugendlehre an. Gemäss Aristoteles stellt die sogenannte 'Mitte' zwischen den Extremen einen Wert beziehungsweise eine Tugend dar. Auch wenn Aristoteles viele dieser Tugenden benennt - wenn Feigheit der Mangel und Tollkühnheit das Übermass darstellt, dann ist Mut die Mitte -, erwähnt er in der Tat mehrmals, dass viele Tugenden keine Namen besitzen, was relativ plausibel erscheint. So ist es denkbar, dass Extreme wie übersteigerter Ehrgeiz oder extreme Faulheit im Gegensatz zur Tugend in der Mitte benannt werden können. In diesem Fall kann man sagen, dass es einen Wert darstellt, dass man weder übermässig ehrgeizig noch vollkommen faul ist, und die Tugend irgendwo dazwischen liegt. Auch ist es denkbar, wie Chang festhält, dass Werte in unseren Gedanken existieren und erst zu einem späteren Zeitpunkt benannt werden. Bereits vor sechzig Jahren waren beispielsweise Frauen am Arbeitsplatz mit Mangel an Respekt, Chauvinismus oder körperlichen Belästigungen konfrontiert. Heute werden diese Dinge unter dem Namen der sexuellen Belästigung vereint.

Das Problem ist, dass sich überhaupt nicht beurteilen lässt, ob die Position der namenlosen Deckwerte überzeugend ist. Ich meine, dass man etwas über den Inhalt dieses Konzepts wissen müsste, um beurteilen zu können, ob die Idee plausibel ist. Bei Aristoteles steht 
dahinter eine ganze Theorie, was eine Tugend ausmacht. Changs Erwiderung darauf' (...) the fact that we cannot explicate the content of a concept does not mean that there is no such concept or that we do not possess it. (...)' erscheint unplausibel (Chang 2004: 18). In diesem Zusammenhang sollte der Inhalt eines zentralen Konzepts erläutert werden. Wenn Simon fragt: 'Was ist sowohl wichtiger als das Versprechen gegenüber dem Lehrer als auch wichtiger als Annas Bestehen der Matura?', muss die Antwort einen Inhalt (content) aufweisen.

Es könnte eingewendet werden, dass mit einem Deckwert eine andere Idee, nämlich kein zusätzlicher Wert, gemeint ist und ich von einem falschen Verständnis ausgehe.

Betrachten wir zu diesem Zweck den Deckwert der sexuellen Belästigung. Nehmen wir an, dass eine Arbeitnehmerin schlechte Erfahrungen mit ihren Vorgesetzten gemacht hat. Ein Vorgesetzter erzählte ihr sexistische Witze. Ein anderer hingegen drohte ihr implizit mit der Kündigung, wenn sie mit ihm nicht privat Kontakt habe. Man könnte argumentieren, dass der Deckwert der sexuellen Belästigung in diesem Fall nicht etwas grundlegend Drittes darstellt. Vielmehr, so versteht es Chang, stellt er einen Überbegriff für diese unterschiedlichen Dinge dar, mit dem Zusatz, dass ein Eingriff in die Privatsphäre eine gröbere Belästigung als ein sexistischer Witz darstellt. Das heisst, dass sich der Überbegriff der sexuellen Belästigung aus der Summe seiner Komponenten zusammensetzt, sexistische Witze, Eingriffe in die Privatsphäre... und den einzelnen Komponenten unterschiedliche Normativität zuspricht: Der Eingriff in die Privatsphäre ist schlimmer als ein sexistischer Witz.

In Simons Beispiel liegt allerdings kein solcher Deckwert vor. Das Versprechen gegenüber dem Lehrer und Annas potenzielle Freude über die bestandene Matura sind zu unterschiedlich, als dass es dafür einen gemeinsamen Überbegriff gäbe. Nehmen wir aber an, dass ähnlich wie im Falle der sexuellen Belästigung zum jetzigen Zeitpunkt lediglich noch keine Name dafür existiert. Falls tatsächlich ein solcher Deckwert existierte, könnte der harte Fall damit tatsächlich gelöst werden. Aber eben: würde, hätte, könnte. Selbst wenn ein solcher Überbegriff existieren würde, ist es nicht dasjenige, wonach Simon sucht. Er will vielmehr wissen, ob es etwas Drittes gibt, das sowohl wichtiger ist als das Versprechen gegenüber dem Lehrer als 
auch wichtiger als Annas Bestehen der Matura, etwas Grundlegendes, zu dem die beiden Dinge unterschiedlich beisteuern. Insbesondere mit einem solchen Deckwert, der nicht vorhanden zu sein scheint, liesse sich der harte Fall beziehungsweise die evaluative Unwissenheit auflösen.

\subsection{Zusammenfassung}

Erstens wurde die Klumpentheorie eingeführt und interpretiert. Diese Theorie besagt (vereinfacht dargestellt), dass zwei Gegenstände genau dann unvergleichbar sind, wenn kein Deckwert existiert, hinsichtlich dessen die beiden Gegenstände verglichen werden können.

Zweitens wurde gezeigt, dass diese Vergleichstheorie wichtige Fragen unbeantwortet lässt (Was ist ein Deckwert? Was muss erfüllt sein, dass bei einem Vergleich ein Deckwert zur Verfügung steht?) und auf einem unplausiblen Begriff von Vergleichbarkeit basiert (Vergleichbarkeit setzt keine Quantifizierung voraus).

Drittens wurde argumentiert, dass harte Fälle tatsächlich anhand eines Deckwerts aufgelöst werden könnten, wenn darunter ein basaler Wert verstanden würde. Ein solcher Deckwert wäre grundlegender und wichtiger als die unterschiedlichen Qualitäten, deren Wichtigkeit dann dadurch beurteilt werden könnte, wie stark sie den basalen Wert befördern. Dabei zeigte ich, dass ein Akteur im Falle von Unvergleichbarkeit von diesem Konzept nicht Gebrauch machen kann und auch Changs Ansatz der namenlosen Werte nicht überzeugt.

Trotzdem - rein pragmatisch betrachtet - muss ein Akteur irgendeine unvergleichbare Option wählen. Davon handelt das letzte Kapitel. 
Kapitel 10

Weshalb nicht

beliebig wählen? 
In den letzten Kapiteln wurde gezeigt, dass bestehende Strategien, harte Fälle als vergleichbar auszuweisen, nicht Erfolg versprechend sind. Dennoch stellt sich die Frage, was im Falle von unvergleichbaren Handlungsoptionen zu tun ist. In diesem Kapitel geht es also nicht mehr darum, inwiefern Unvergleichbarkeit ein Problem oder eben keines für die Rationalität darstellt. Im Zentrum steht die normative Frage, wie mit Unvergleichbarkeit umgegangen werden soll. Der Fokus wird pragmatischer ausgerichtet: 'Was tun wir jetzt?' Meine Idee, mit obiger sehr allgemeinen Fragestellung umzugehen, ist es, einen Teilaspekt zu behandeln und sie im folgenden Sinn einzuschränken: Dürfen wir im Falle von unvergleichbaren Optionen beliebig wählen? Unabhängig davon, wie eine beliebige Wahl genau spezifiziert wird, scheint sie nicht mit unserem Selbstverständnis verträglich, autonome Wesen sein zu wollen. Damit ist gemeint, dass wir in der Regel selbst über unser Leben entscheiden möchten, insbesondere, wenn es um wichtige Entschlüsse geht. Die intuitive Antwort auf die Möglichkeit, beliebig zu wählen, lautet dementsprechend: 'Nein, eher nicht willkürlich entscheiden.'

Dieser Standpunkt wird von einigen Philosophen vertreten. Pinkowski und Schaber beispielsweise gehen davon aus, dass die Entscheidung zwischen unvergleichbaren Optionen nicht dem Zufall überlassen wird oder überlassen werden sollte. ${ }^{1}$ Beide nennen dafür jedoch kein Argument. Pinkowski etwa nennt keine Begründung, weshalb Unvergleichbarkeit nicht zufällig aufgelöst werden soll, sondern appelliert an unsere Intuition, weil der Zufall bei wichtigen Entscheidungen keine adäquate Strategie darstellt. Und auch Chang vertritt die Position, dass wir in solchen Fällen nicht zufällig entscheiden sollen. ${ }^{2}$ Sie meint, dass wir uns zu einer Optionen bekennen sollten. Ich werde versuchen, Changs Position in dem Sinne zu plausibilisieren, dass wir eine existenzielle Stellungnahme vornehmen sollten ('Ich sehe mich als Musiker').

Je mehr ich allerdings über die Frage nachdachte, weshalb Unvergleichbarkeit nicht beliebig aufgelöst werden darf, desto weniger liess sich die Intuition aufrechterhalten. Ich plädiere im Folgenden 
für die These, dass es bei einem bestimmten Typus von Unvergleichbarkeit schlicht keine Rolle spielt, ob beliebig, aufgrund einer nichtbegründeten Neigung ('die Alternative scheint irgendwie doch besser zu sein') oder eines Bekenntnisses entschieden wird.

Es ist wichtig zu erwähnen, dass es in diesem Kapitel nicht um eine empirische These geht. Schaber beispielsweise denkt, dass wir es als unangemessen ansehen, im Falle von Unvergleichbarkeit - er spricht von ungefähr gleich guten Optionen, aber es gilt in diesem Kontext auch für Unvergleichbarkeit ${ }^{3}$ - anhand eines Münzwurfs zu einer Entscheidung zu gelangen (vgl. hierzu aber Reuter und Messerli 2017). Er schreibt:

'Das bedeutet aber nicht, dass sich "genau gleich" und "ungefähr gleich" nicht unterscheiden lassen. Es handelt sich nämlich jeweils um unterschiedliche Formen der Indifferenz. Bei zwei genau gleich wertvollen Optionen wären wir ohne zu zögern bereit, eine Münze zu werfen. Das trifft auf ungefähr gleich wertvolle Optionen nicht zu. Bei solchen Optionen halten wir das Werfen einer Münze für unangemessen.' (Schaber 1994: 158).

Meine These jedoch ist normativ: Man darf beliebig wählen. Die These, dass man nicht beliebig wählen soll, ist falsch.

Das Kapitel besteht aus vier Teilen: Erstens wird das Konzept einer beliebigen Wahl diskutiert. Zweitens zeige ich, weshalb beliebig gewählt werden darf. Vereinfacht gesagt deshalb, weil sich die Wahl zwischen unvergleichbaren Optionen nur durch Erfahrung und ein Ausprobieren als 'richtig' oder 'falsch' erweisen kann. Drittens lege ich dar, wann beziehungsweise unter welchen Bedingungen beliebig entschieden werden darf. Ich grenze die Gültigkeit der These ein. Viertens werden mögliche Argumente diskutiert und zurückgewiesen, weshalb im Falle von Unvergleichbarkeit nicht beliebig gewählt werden darf. 


\subsection{Beliebig wählen}

Wir müssen uns an dieser Stelle in Erinnerung rufen, dass es charakteristisch für Unvergleichbarkeit ist, dass die relevanten Werte nicht gegeneinander abgewogen werden können. Man befindet sich in einem Zustand, den ich in Abgrenzung zu faktischer Unwissenheit als sogenanntes evaluatives Unwissen bezeichnet habe. Das heisst, dass die Wahl zwischen unvergleichbaren Optionen nicht auf der Basis einer Präferenz, im Sinne einer Evaluation, welche Option alles in allem besser ist, erfolgen kann.

Verstehen wir nun unter einer beliebigen Entscheidung eine Wahl, welche nicht auf der Basis einer begründeten Evaluation erfolgt, existiert schlicht keine Alternative. Das beliebige Wählen ist dann insofern alternativlos, weil Unvergleichbarkeit beinhaltet, dass es nicht möglich ist, aufgrund der besten Gründe beziehungsweise einer Wertung zu entscheiden. Daraus folgt, dass es unangemessen wäre, eine nicht-beliebige Entscheidung zu erwarten. Man könnte auch sagen, dass die These nicht haltbar ist, dass unvergleichbare Optionen nicht beliebig aufgelöst werden sollen, weil aus dem pragmatischen Grundsatz, dass Sollen in der Regel Können voraussetzt $(A \rightarrow B)$, verbunden mit 'nicht-können' $(\neg B)$ auch 'nicht-sollen' $(\neg A)$ folgt. Interessant wird es deshalb erst dann, wenn es um verschiedene Strategien geht, welche befolgt oder abgelehnt werden können. Eine Möglichkeit besteht darin, eine beliebige Wahl als sogenanntes picking zu deuten. In ihrem Aufsatz 'Picking and Choosing' verstehen Ulmann-Margalit und Morgenbesser beliebiges Wählen - in Abgrenzung zu choosing - als picking (Ulmann-Margalit und Morgenbesser 1977). Wir können picking so verstehen, dass man nicht auf der Basis von relevanten Gründen, sondern auf der Basis eines willkürlichen Faktors wählt. Zum Beispiel: 'Der FC Bayern hat gegen Dortmund verloren, und deshalb studiere ich Ökonomie und nicht Jurisprudenz.' Abstrakter und idealisiert formuliert, heisst dies Folgendes: Wenn im Falle von Unvergleichbarkeit der Grund $a$ für die eine und der Grund $b$ für die andere Option spricht und man nicht weiss, welcher wichtiger ist, darf auf der Basis eines willkürlichen Faktors $c$ (FC Bayern) irgendeine der beiden Optionen gewählt werden. Man trifft die Wahl ohne guten Grund. 
Eine andere Möglichkeit lautet, unter einer beliebigen Wahl einen realen Münzwurf oder ein tatsächliches Würfeln zu verstehen. Harvey Dent alias Two-Face aus den Batman-Comics trifft seine Entscheidungen auf diese Weise. Sobald Two-Face sich entscheiden muss, wirft er seine Münze.

Worin besteht die Differenz zwischen den beiden Deutungen? Der Unterschied zwischen picking und Würfeln liegt darin, dass nur bei einem (nicht gezinkten) Würfel, einem Münzwurf oder etwas Ähnlichem mit Sicherheit gesagt werden kann, dass es sich dabei um ein zufälliges Verfahren handelt. Wir wissen aus der Psychologie, dass wir sehr vielen unbewussten Beeinflussungen (sogenannten biases) ausgesetzt sind. ${ }^{4}$ Bei einem realen Münzwurf kann im Gegensatz zum picking ausgeschlossen werden, dass aufgrund einer solchen unbewussten Neigung entschieden wird. Ich denke notabene nicht, dass wir uns bezüglich unserer Handlungsmotive stets täuschen und es nicht auch beim picking möglich ist, zufällig zu wählen. In diesem Punkt folge ich Henning (2015). Dieser argumentiert, dass Ärzte in Dilemma-Situationen keine statistischen Verfahren benutzen müssen, sondern auch picking betreiben dürfen. Die Idee, welche breite Zustimmung findet und gegen die Henning argumentiert, besagt, dass bestimmte moralische Dilemmata durch einen stochastischen Prozess aufgelöst werden müssen. Die Idee ist, dass nur auf diese Weise problematische Neigungen ausgeschlossen werden können. So ist es denkbar, dass aufgrund der unbewussten Neigung, attraktivere Menschen besser zu behandeln, schöne Menschen mehr Organe erhalten. Gemäss Henning können die Ärztinnen aber auch durch picking attraktiven und weniger attraktiven Menschen die gleichen Chancen einräumen. Ich folge ihm wohlgemerkt nur in diesem Punkt. Trotzdem ist man nämlich nur bei

4 So zeigt z.B. Wilson in seinem Buch Strangers to Ourselves, dass wir oft ein zu optimistisches Bild von uns selber haben (Wilson 2002). Wilson geht davon aus, dass unbewusste Prozesse unser Verhalten wesentlich beeinflussen. Man denke auch an das aus der Psychologie bekannte Phänomen der Konfabulation. So ist es beispielsweise bekannt, dass Probandinnen und Probanden in Experimenten zwischen zwei identischen Optionen statistisch signifikant häufiger das rechte Objekt wählen und im Nachhinein Gründe für ihre Entscheidung angeben, die nichts damit zu tun haben, dass sich das Objekt auf der rechten Seite befand. Auch durch den Supermarkt werden wir nach dieser Erkenntnis geführt. Der Eingang z.B. befindet sich normalerweise rechts. 
einem zufälligen Verfahren 'auf der sicheren Seite', dass kein unbewusster Faktor die Entscheidung beeinflusst. Henning scheint nicht zeigen zu können, dass das picking bei der Auflösung moralischer Dilemmata gegenüber dem stochastischen Verfahren irgendwelche Vorteile aufweist.

Inwiefern wäre es aber im Falle von Unvergleichbarkeit, die nicht unbedingt mit Ethik zu tun haben muss, überhaupt 'schlimm', wenn ein unbewusster Faktor die Entscheidung beeinflusste?

Problematisch wäre zum Beispiel, wenn die unbewusste Neigung im Widerspruch zu unserem besten Urteil stände, man $x$ für die beste Option hält und aufgrund der unbewussten Neigung $y$ wählt. Ein Beispiel: Man denkt, dass es das Beste ist, Max-Havelaar-Kaffee zu kaufen, und entscheidet sich aufgrund der Farbe der Verpackung oder der Lage im Regal für ein anderes Produkt. Im Falle von Unvergleichbarkeit ist eine solche Evaluation $-x$ stellt die beste Option dar - aber ohnehin nicht möglich. Plausibler ist es deshalb anzunehmen, dass die unbewusste Beeinflussung genau dann problematisch ist, wenn sie vom Akteur eigentlich vermieden werden möchte oder sollte. Ein mögliches Beispiel stellt unser Hang dar, den Status quo gegenüber Veränderungen zu bevorzugen. Es ist denkbar, dass ein Akteur diese Tendenz vermeiden respektive offener für Neues sein möchte und deshalb eher würfeln sollte, da sogenannte (unerwünschte) Risikoaversion hierbei keine Rolle spielt.

Die Medaille hat aber auch eine Kehrseite: Unbewusste Beeinflussungen können auch positiv sein, in dem Sinne, dass sie Dinge beinhalten können, die uns wichtig, aber bewusst nicht zugänglich sind. Wenn man wie Two-Face eine Münze wirft, fällt dieser positive Aspekt des Unbewussten weg.

Halten wir fest: Unbewusste Beeinflussungen können negativ, positiv oder neutral sein, in dem Sinne, dass man beispielsweise Wörter mit 'A' mag und deshalb nach Australien und nicht nach Zypern in die Ferien fährt. Die beiden Deutungen einer beliebigen Wahl (würfeln und picking) unterscheiden sich hinsichtlich solcher unbewusster Beeinflussungen. Durch das Würfeln werden bei der Entscheidung sämtliche unbewussten Neigungen, ob positiv, negativ oder neutral, ausgeschlossen. Durch das picking nimmt man bei einer Entscheidung sämtliche unbewussten Neigungen in Kauf. 
Die Intuition, dass die Entscheidung zwischen unvergleichbaren Optionen nicht dem Zufall überlassen werden sollte, richtet sich wohl stärker gegen das Würfeln als gegen das picking. Betrachten wir hierzu folgende drei Fälle:

Fall 1: Eine Akteurin entscheidet sich aufgrund von picking für die Mitgliedschaft im Hockeyverein und gegen das Singen im Chor. Fall 2: Eine Akteurin wirft eine Münze, ob sie Hockey spielen oder im Chor singen will.

Fall 3: Eine Akteurin führt auf Facebook eine Online-Umfrage durch, was sie tun soll.

Intuitiv am abwegigsten erscheint den meisten wohl der dritte Fall, in dem die Akteurin aufgrund fremder Präferenzen wählt. ${ }^{5} \mathrm{Am}$ wenigsten abwegig hingegen erscheint den meisten wohl der erste Fall, in dem die Akteurin beliebig, aber immer noch 'selbst' entscheidet. Und das mittlere Übel stellt intuitiv wohl der zweite Fall dar. Diese mögliche Lagerung der Intuitionen kann unter Umständen damit erklärt werden, dass wir im ersten Fall noch am ehesten davon sprechen würden, dass die Akteurin selbst wählt. Letztlich muss die Akteurin aber in allen drei Fällen selber entscheiden. Damit meine ich, dass sich die Akteurin immer noch entscheiden muss, ob sie den Münzwurf oder die Umfrage als Entscheidungskriterium akzeptiert. Sie muss sich ja nicht zwingend daran halten und kann ex post immer noch das Gegenteil tun.

An dieser Stelle mag eingewendet werden, dass es falsch ist, dass man im Falle des picking unbewussten Neigungen erliegt und deshalb schlechtere Entscheidungen trifft. Die Idee ist, dass solche Neigungen bereits auf den Erfassungsprozess der fraglichen Optionen zutrifft beziehungsweise bereits Teil der diagnostizierten Unvergleichbarkeit sind. Vereinfacht gesagt: Die Werte, die nicht verglichen werden können, werden nicht neutral bewertet. Gemeint ist, dass diese Einschätzung ebenfalls bereits auf unbewussten Prozessen basiert. Das 
mag sein. Wir wissen aus der Psychologie, dass es oft nicht Werte oder Gründe, sondern andere Dinge, etwa die Kleidung oder der Geruch einer anderen Person, sind, welche unsere Entscheidungen beeinflussen. Aber dieser potentielle Einwand ist nicht relevant.

Der Grund dafür ist, dass es für die normative These - zwischen unvergleichbaren Optionen darf beliebig gewählt werden - letztlich keine entscheidende Rolle spielt, von welcher Variante einer beliebigen Wahl ausgegangen wird. Das Argument, das ich im nächsten Teil einführe, liefert für beide Versionen gleich viel Evidenz. ${ }^{6}$

\subsection{Gründe für das beliebige Wählen}

Das Argument orientiert sich an einem bestimmten Konzept praktischer Rationalität. Genauer orientiere ich mich an Millgrams Theorie der sogenannten 'praktischen Induktion'. In der Wissenschaft meint Induktion, stark vereinfacht gesagt, dass man aufgrund von Einzelfällen und einzelnen Beobachtungen zu Verallgemeinerungen kommt. Millgram argumentiert, dass es im praktischen Bereich ein Analogon zur Induktion gibt, nämlich Erfahrung. Das Lernen aus Erfahrung sollte innerhalb der praktischen Rationalität diejenige Rolle einnehmen, welche die Beobachtung in den empirischen Wissenschaften spielt. Mithilfe von Erfahrung, so Millgram, lassen sich aus eher spezifischeren Urteilen allgemeinere Urteile herleiten. Er nennt folgendes Beispiel: 'Als ich jünger war, habe ich gemeint, Höflichkeit spiele keine besonders grosse Rolle. Doch jetzt, da mir genug Fälle von schlechten Manieren untergekommen sind, um einzusehen, wie viel Höflichkeit aus macht, bin ich der Meinung, sie sei eben doch wichtig. In diesem Fall habe ich etwas Allgemeines über Dinge, die eine Rolle spielen, gelernt, und gelernt habe ich es aus Einzelerfahrungen.' (Millgram 1997: 52, 53). Mit diesem Gedanken will er alternative Sichtweisen praktischer Rationalität, wie eine rein instrumentelle Auffassung praktischer

6 Philosophinnen und Philosophen, die denken, dass beliebiges Wählen im Sinne von picking (FC Bayern) nicht möglich ist, sollen die beliebige Wahl im Sinne eines stochastischen Verfahrens interpretieren. Man könnte nämlich argumentieren, dass ein Akteur beispielsweise implizite Erwartungen hat, ob der FC Bayern gegen Dortmund verlieren wird, und es deshalb keine beliebige Wahl darstellt. 
Vernunft, ad absurdum führen. Letztere verkennt unter anderem, so Millgram, dass man nur durch Erfahrung zu neuen Zielen kommen kann. Millgrams Hauptthese, dass nur Erfahrung einem zeigt, worauf es ankommt, und man primär durch Erfahrung lernt, was einen zufrieden beziehungsweise unzufrieden macht, scheint mir plausibel zu sein. ${ }^{7}$

Es ist wichtig zu betonen, dass es nicht um eine Übernahme und Verteidigung von Millgrams Ansatz praktischer Vernunft geht. Für uns ist nur ein ganz spezifischer Aspekt von Millgrams Theorie relevant, nämlich seine Ausführungen über den Hedonismus, und weshalb darin das Wesen der Lust falsch verstanden wird: 'Hedonists assume that because desire and goals change in response to experienced pleasure and displeasure, these must be the actual goals. But this view is naive: pleasure and displeasure are indicators and signs of desirability we use in determining what our goals should be.' (Millgram 1997: 117). Unter Lust versteht Millgram ein Gefühl, dass man etwas gerne tut, es keine Anstrengung erfordert, auch wenn es eventuell schwierig ist (Millgram 1997: 109).

Anstatt von Lust zu sprechen, benutze ich aber lieber den Begriff des Wohlergehens, da es nicht um blosse Bedürfnisbefriedigung geht. ${ }^{8}$

Ich will aber nicht behaupten, dass persönliche Erfahrung notwendig ist, um die Güte einer Handlung beurteilen zu können. Man muss nicht selbst gefoltert haben, um zu wissen, dass man dies auf keinen Fall tun will. Millgram ist in diesem Punkt sehr radikal. Er kann so gelesen werden, dass die Güte einer Handlung nur durch Erfahrung und z.B. nicht durch Vorstellungskraft als richtig oder falsch beurteilt werden kann (Millgram 1997: 151, 152). Ich denke, auch wenn an dieser Stelle nicht darauf eingegangen werden kann, dass Wohlergehen eng mit Selbstachtung und Integrität zusammenhängt. Badhwar schreibt: 'Most contemporary philosophers of well-being who take their inspiration from ancient ethics hold that well-being requires virtue, and that there's a necessary connection between the idea of the individual's well-being an the idea of her moral goodness.' (Badhwar 2014: 13). Dass tatsächlich eine notwendige Verbindung zwischen Moral, Selbstachtung und Wohlergehen existiert, ist eine starke These. Grundsätzlich erscheint mir ein solcher Ansatz jedoch plausibel. Sehr vereinfacht gesagt, ist mit Selbstachtung gemeint, dass man sich mit seinen Wünschen identifizieren kann. Umgangssprachlich spricht man in diesem Zusammenhang manchmal davon, dass man 'nicht mehr in den Spiegel schauen kann'. Dies trifft es relativ gut: Wenn man sich mit seinen Wünschen identifiziert, kann man 'in den Spiegel schauen'. Man fühlt sich im Reinen mit sich selbst. Integrität stellt, sehr vereinfacht gesagt, die Übereinstimmung von Handlung und moralischer Überzeugung dar. Sowohl die Missachtung von Integrität als auch von Selbstachtung, so denke ich, kann auf Dauer nicht zu Wohlergehen führen. Wir können uns zum Teil noch Jahre danach erinnern, wenn wir entgegen unserer Selbstachtung gehandelt haben. Dies ist für diejenigen Fälle von Unvergleichbarkeit wichtig, welche moralische Überlegungen beinhalten. Aber ich werde im nächsten Abschnitt darlegen, weshalb wir diese Fälle separat untersuchen müssen. 
Wohlergehen stellt ähnlich wie Lust kein konkretes Ziel dar. Wenn man jemandem sagt: 'Erhöhe dein Wohlergehen!', hat diese Person wie im Falle von 'Werde glücklich!' vermutlich keine Ahnung, was sie damit anfangen soll. Wohlergehen, so die These, ist wie Lust vielmehr ein Indikator, dass man die richtige Wahl getroffen hat. Wenn man die richtige Wahl trifft, steigert dies ceteris paribus das Wohlergehen. Wenn man die falsche Wahl trifft, vermindert dies ceteris paribus das Wohlergehen. Wohlergehen, so kann man sagen, stellt eine Begleiterscheinung einer beliebigen Tätigkeit, eine Form der Zufriedenheit dar, die sich einstellt oder ausbleibt, wenn man Ziele verfolgt und Dinge tut. So kommen Wohlergehen wie Lust - im Gegensatz zu den Gründen, die für oder gegen die einzelnen Optionen sprechen denn auch nicht selbst im Deliberationsprozess vor (vgl. Millgram 1997: 119).

Was hat dies damit zu tun, dass gerechtfertigterweise beliebig gewählt werden darf? Weshalb stellt der Wert, aus neuen Erfahrungen zu lernen, ein Argument für das beliebige Wählen dar?

Der Grund ist, dass sich die Unvergleichbarkeit nur durch ein Hineindenken und letztlich ein Ausprobieren beziehungsweise durch Erfahrung 'auflösen' lässt. Aus welchen absurden Motiven heraus die Erfahrung gesammelt wird, zum Beispiel, weil der FC Bayern gegen Dortmund verloren hat, ist im Grunde genommen egal. Dass man die Erfahrung macht, darum geht es. Am grünen Tisch zu beurteilen, wie das Leben unter den unterschiedlichen Bedingungen sein wird, ist schlicht nicht möglich. Wie in der Einleitung festgehalten worden ist: Wenn man einen Monat, nachdem man mit dem Hockeyspielen aufgehört hat, realisiert, dass sich das Wohlergehen verschlechtert, man irgendwie unzufrieden ist, stellt dies einen Indikator dar, dass es die falsche Entscheidung war, im Chor mitzumachen. Gegebenenfalls kann die Entscheidung dann rückgängig gemacht werden. Das Training kann wieder aufgenommen oder das Singen im Chor wieder abgebrochen werden. Inwiefern eine Entscheidung gut oder schlecht ist, kann gemäss diesem Ansatz nicht punktuell beurteilt werden. Es kann sich nur mit der Zeit zeigen. Die Idee besteht im Grunde genommen also darin, dass die 'Auflösung' einer evaluativen Unwissenheit in der Regel einen Lernprozess voraussetzt, um zu erfahren, was zählt. 
Wir können diese Überlegung in Form eines Arguments wie folgt darstellen:

1. Ein harter Fall kann als evaluative Unsicherheit interpretiert werden.

2. Wohlergehen ist entscheidend in einem harten Fall.

3. Wenn (1) und (2) richtig sind, dann ist es zentral, mehr Erfahrungen zu sammeln.

4. Wenn es zentral ist, mehr Erfahrungen zu sammeln, dann ist eine zufällige Wahl gerechtfertigt.

$\therefore \quad$ Es ist gerechtfertigt, in harten Fällen zufällig zu wählen.

Ich habe die Richtigkeit der ersten Prämisse in Kapitel 3 belegt. Die zweite Prämisse sagt, dass Wohlergehen eine wichtige Rolle spielt. Die dritte Annahme behauptet, dass wenn (1) und (2) richtig sind, mehr Erfahrungen den Konflikt auflösen können. Prämisse 4 basiert auf der Idee, dass es keine Rolle spielt, wie man die Entscheidung trifft, weil das ausschlaggebende Element erst nach der Wahl passiert. Es ist die Erfahrung, die man machen wird.

Das Argument sagt wohlgemerkt nicht, dass Rationalität fordert, dass man beliebig wählen soll. Ich sehe kein gutes Argument für diese stärkere These. Das Argument besagt, dass man beliebig wählen darf.

Dahinter steht die Idee, dass es zentral ist, dass uns Unvergleichbarkeit nicht blockiert. ${ }^{9}$ Aber es ist wichtig zu sehen, dass es harte Fälle gibt, in denen die Entscheidung gegebenenfalls nicht aufgrund von Wohlergehen getroffen werden sollte. Mögliche Beispiele sind sogenannte moral-prudential conflicts (Moral oder Glück?) oder moralische Dilemmata. Ich werde im nächsten Teil auf moralische Dilemmata genauer eingehen und die These weiter präzisieren. spielsweise Entscheiden besser als Nicht Entscheiden ist. Genauer, da Nicht-Entscheiden in einem gewissen Sinne auch Entscheiden ist, bleibe ich die Antwort schuldig, weshalb die Wahl einer unvergleichbaren Option besser ist, als nichts zu tun. Wenn zwei Studiengänge unvergleichbar sind, könnte man ja auch gar nichts studieren. Die Beantwortung dieser Frage sprengt den Rahmen dieses Buches. 


\subsection{Bedingungen für das beliebige Wählen}

In diesem Unterkapitel geht es darum, die These von benachbarten Strategien abzugrenzen und wichtige Einschränkungen ihrer Gültigkeit vorzunehmen. Es geht darum, die Bedingungen herauszuarbeiten, wann die These stichhaltig ist. Weshalb jedoch gilt das Argument des letzten Kapitels unter diesen Bedingungen nicht? Es ist wichtig zu sehen, dass ich dies nicht behaupte. Beispielsweise sage ich nicht, dass faktische Unsicherheit Randomisierung per se ausschliesst. Es geht darum, dass die Fälle, die im Folgenden diskutiert werden, Spezialfälle darstellen, die separat untersucht werden müssen.

\section{Welcher Typ von Unvergleichbarkeit?}

Erstens: Die These gilt nicht für alle Typen von Unvergleichbarkeit. Ich sage nicht, dass bei jedem Typ von Unvergleichbarkeit beliebig gewählt werden darf.

Auch wenn Unvergleichbarkeit, so wie das Konzept in diesem Buch entwickelt wurde, primär auf Wertkonflikte und evaluatives Unwissen zurückgeführt werden kann, spielt faktische Unsicherheit dabei eine Rolle, wie mit Unvergleichbarkeit umgegangen werden soll. Denken wir an die Berufs- oder Studienwahl. Auch wenn es evaluatives Unwissen ist, das die unterschiedlichen Studiengänge unvergleichbar macht, liefert faktische Unsicherheit eine Begründung, weshalb nicht gewürfelt werden sollte. Diese besagt, dass die Beschaffung zusätzlicher Informationen vielleicht einen Unterschied in der Evaluation ausmachen kann (vielleicht ist ja doch irgendeine Option besser). So ist es denkbar, dass eine Akteurin (i) nicht weiss, wie wichtig ihr ein besseres Verständnis wirtschaftlicher oder juristischer Zusammenhänge ist (evaluatives Unwissen), (ii) deshalb der eine Studiengang weder besser noch schlechter als noch gleich gut wie der andere Studiengang ist (Unvergleichbarkeit), aber (iii) die Akteurin dennoch ein Urteil zugunsten von Jurisprudenz fällen kann, je mehr sie darüber erfährt, was sie im Studium der Ökonomie erwarten wird, wie beispielsweise, dass der Fokus nicht auf dem Verstehen aktueller Ereignisse (etwa der Finanz- oder Eurokrise), sondern auf dem Rechnen mit und Interpretieren von abstrakten Modellen liegt. 
Je nachdem, ob faktische Unsicherheit involviert ist oder nicht, sollte mit Unvergleichbarkeit also anders umgegangen werden. Genauer formuliert, lautet die These nicht, dass Unvergleichbarkeit, die faktische Unsicherheit beinhaltet, durch einen Münzwurf aufgelöst werden darf. Anstatt zu würfeln, kann in diesem Fall beispielsweise versucht werden, mehr Informationen zu beschaffen. So kann man als Hörer verschiedene Ökonomieveranstaltungen besuchen, bevor man sich für das eine oder andere Studium entscheidet, wobei sogenannte Opportunitätskosten natürlich zu berücksichtigen sind. Damit ist gemeint, dass die Informationsbeschaffung selbst Zeit benötigt, die man auch anders hätte verbringen können.

Obige Situationen stellen in dem Sinne Spezialfälle dar, als eine alternative Option $z$ existiert, welche besser als beide unvergleichbaren Optionen $x$ und $y$ ist. Im obigen Beispiel besteht diese Alternative darin, mehr Informationen zu beschaffen. Mir geht es jedoch um Fälle, in denen keine dritte und bessere Option $z$ existiert. In diesem Fall wäre die Beschaffung zusätzlicher Informationen etwa unmöglich oder es könnte ausgeschlossen werden, dass mehr Information irgendetwas bei der Evaluation ändern würde.

In Kapitel 3 wurde argumentiert, dass es diese Fälle von Unvergleichbarkeit gibt und nicht jeder Typ harter Fall faktische Unsicherheit beinhaltet. Bei dieser Form der Unvergleichbarkeit spielt faktische Unsicherheit in dem Sinne keine Rolle, als die Beschaffung zusätzlicher faktischer Informationen irrelevant ist. Als Beispiel eines Falles, der (i) wichtig ist, (ii) evaluatives Unwissen, aber (iii) keine faktische Unsicherheit involviert, wurde folgender Kasus angeführt: Ein Akteur muss die Entscheidung treffen, ob er dreimal pro Woche ins Hockeytraining gehen oder diese Zeit anderweitig verbringen, zum Beispiel öfters mit Freunden Bier trinken will.

Die These ist, dass bei diesen Fällen von Unvergleichbarkeit, also auch beim obigen Beispiel, gewürfelt werden darf.

\section{Achtung Moral}

Zweitens: Die These gilt nicht für Entscheidungen, bei denen Wohlergehen gegebenenfalls zweitrangig ist. Betrachten wir hierzu moralische Dilemmata. Was sind genuine moralische Dilemmata 
(sofern es sie denn gibt, was mir plausibel scheint)? Darunter können wir Situationen verstehen, in denen man $x$ tun soll $(O x)$ und $y$ tun soll $(O y)$ und sich $x$ und $y$ nicht zusammen realisieren lassen $(\neg C(x \wedge y))$.

Weshalb ist diese Abgrenzung wichtig? Dafür sprechen zwei Punkte: Einerseits ist es eine offene Frage, ob moralische Dilemmata unter die Extension von Unvergleichbarkeit fallen. Andererseits - angenommen, sie sind Teil der Definition - ist es eine offene Frage, ob sie in diesem Fall nicht extra behandelt werden müssten.

Wir können sagen, dass die Abgrenzung wichtig ist, weil dadurch folgendes Problem ausgeschlossen werden kann: Man behauptet, dass im Falle von Unvergleichbarkeit gewürfelt werden darf, und moralische Dilemmata, die unter Umständen unter die Definition von Unvergleichbarkeit fallen, legen nahe, dass nicht gewürfelt werden sollte. Beispielsweise kann man argumentieren, dass deshalb nicht beliebig gewählt werden darf, weil es davon abhängt, welche ethische Theorie richtig ist.

Ich denke jedoch, dass gewisse Typen von moralischen Dilemmata zeigen, dass die generelle These, wichtige Entscheidungen dürften nicht zufällig aufgelöst werden, falsch ist (Pinkowskis Meinung). Denken wir an Eltern, deren Zwillinge an einem identischen Gendefekt leiden, der die Funktion der Leber stört. Die Zwillinge sterben, wenn sie nicht bald ein neues Organ erhalten. Bislang wurde allerdings erst ein Spender mit geeigneter Leber gefunden. Bleibt die Suche der Eltern nach einem weiteren Spender erfolglos, müssen sie die Entscheidung treffen, welches Kind überlebt und welches stirbt. Hierbei würde wohl niemand behaupten wollen, dass die Eltern selbst entscheiden müssen und sich vor der Entscheidung 'drücken', wenn sie dies den Ärzten überlassen. Und niemand würde den Ärzten einen Vorwurf machen, wenn sie dabei ein randomisiertes Verfahren benutzen oder einfach beliebig entscheiden. Man könnte sogar dafür argumentieren, dass eine Ärztin in diesem Fall die Pflicht hat, bei der Entscheidungsfindung ein zufälliges Verfahren zu wählen oder einfach beliebig zu 'picken', da jeder Zwilling eine fünfzigprozentige Überlebenschance besitzen sollte. Es existieren aber auch andere Typen von moralischen oder zumindest anscheinend moralischen Dilemmata, in denen die Gründe, die für die unterschiedlichen Optionen sprechen, sehr unterschiedlich sind (etwa deontologische ver- 
sus utilitaristische Gründe). In diesem Fall scheint es eher falsch zu sein, das Dilemma zufällig aufzulösen. Aber wie erwähnt: Wegen den beiden oben genannten Punkten sind moralische Dilemmata nicht Gegenstand meiner These.

\section{Sequenz von Entscheidungen}

Drittens: Ich behaupte nicht, dass bei einer Sequenz von Entscheidungen zwischen unvergleichbaren Optionen beliebig gewählt werden darf.

Ich sehe gewisse Probleme, wenn man bei einer Abfolge von Entscheidungen zwischen unvergleichbaren Optionen beliebig entscheidet. Zu diesem Zweck können wir die logische Struktur des Arguments der kleinen Verbesserung wieder aufgreifen (vgl. Kapitel 5). Nehmen wir an, dass drei Optionen existieren: 'Hockeytraining' $(x)$; 'Im Chor Singen' $(y)$; 'Hockeytraining + kleiner Geldbetrag' $(x+)$. Die Optionen $x$ und $y$ sind dabei nicht absolut gleich gut, sondern unvergleichbar. Das heisst, dass wenn eine Option marginal verbessert wird, die marginal verbesserte Option $(x+)$ besser als die ursprüngliche $(x)$, nicht aber besser als die andere Option $(y)$ ist.

Die These, dass ein Akteur bei der Wahl zwischen $x+$ und $y$ (Sequenz 1: Entscheidung zum Zeitpunkt $t 1$ ) und zwischen $x$ und $y$ (Sequenz 2: Entscheidung zum Zeitpunkt $t 2$ ) beliebig wählen darf, kann in einem bestimmten Sinne falsch sein. Ich denke, dass folgendes rationales Argument existiert, weshalb in einer Sequenz von Entscheidungen nicht beliebig gewählt werden soll: Nehmen wir an, dass der Akteur erstens $y$ anstatt $x+$ und zweitens $x$ anstatt $y$ wählt. Man kann sich vorstellen, dass sich der Akteur erstens für das Singen im Chor entscheidet. Zweitens macht er die Wahl aber wieder rückgängig beziehungsweise wählt das Hockeytraining, wobei der Trainer nun nicht mehr bereit ist, einen kleinen Geldbetrag zu offerieren. Das heisst, dass der Akteur durch einen Münzwurf Geld verliert. Ich habe Geld gewählt, weil dies in der entscheidungstheoretischen Literatur in ähnlichen Zusammenhängen - ein Akteur wird durch bestimmte Entscheidungen besser oder schlechter gestellt - oft so gehandhabt wird. Es kann aber auch etwas anderes als reales Geld sein, welches das '+' generiert, beispielsweise, dass man bei den Meisterschaftsspielen 
häufiger oder seltener zum Einsatz kommt. Vor dem vermeintlichen Rücktritt konnte man viel und nun nur noch wenig spielen. Diese Variante hat den Vorteil, dass die Annahme, Optionswerte mit Geld $\mathrm{zu}$ addieren, nicht gerechtfertigt werden muss.

Die grundlegende Idee im Argument besteht darin, dass bei einer Sequenz von Entscheidungen zwischen unvergleichbaren Optionen nicht unbedingt gewürfelt werden darf, weil man dadurch am Ende schlechter gestellt werden kann.

Verwirrend kann sein, dass, je nachdem, ob die Entscheidung zum Zeitpunkt $t 1$ stattgefunden hat oder nicht, zum Zeitpunkt $t 2$ einmal gewürfelt werden darf und einmal nicht. Falls die erste Entscheidungssituation niemals existierte ( $x+$ niemals realisierbar war), darf zum Zeitpunkt $t 2$ gewürfelt werden, da man durch die Wahl von $x$ nicht schlechter gestellt wird. Das Argument sagt, dass es zwar im paarweisen Vergleich keine Rolle spielt, ob man $y$ oder $x$ wählt, man bei obiger Sequenz von Entscheidungen in diesem Fall aber $y$ wählen sollte, um keinen Nutzenverlust zu erleiden ( $x+$ wäre auch erhältlich gewesen).

Zwar sind verschiedene Einwände gegen meine Überlegung denkbar - so könnte erwidert werden, dass stabile Präferenzen vorausgesetzt werden oder dass das Argument eher nahelegt, gewisse Entscheidungen zwischen unvergleichbaren Optionen sollten nicht rückgängig gemacht werden oder es dürfe zum Zeitpunkt $t 2$ deshalb beliebig gewählt werden, weil $x+$ nicht mehr erhältlich ist. ${ }^{10}$ Ich denke jedoch, das Argument regt zumindest zum Nachdenken an, dass die Sachlage bei einer Sequenz von Entscheidungen unter Umständen anders beurteilt werden muss. onen nicht beliebig wählt. Zur Erinnerung: $x$ und $y$ (z. B. Anwalt und Künstler) sind unvergleichbar. $x+$ (Anwalt plus zwei Tage Ferien) ist besser als $x$ (Anwalt ohne Ferien). $x+$ (Anwalt plus zwei Tage Ferien) und $y$ (Künstler) sind unvergleichbar. Nehmen wir an, ein Akteur besitzt zuerst $x+$. Zweitens wählt er $y$ anstatt $x+$. Drittens tauscht er $x$ gegen $y$. Viertens bezahlt er einen kleinen Geldbetrag, um $x+$ anstatt $x$ zu erhalten. Nun wählt er anstatt $x+$ wiederum $y$, wählt dann $x$ anstatt $y$ und bezahlt wiederum Geld, um $x+$ gegenüber $x$ zu tauschen ... (ad infinitum). Das heisst, dass man argumentieren kann, dass es besser ist, stets beliebig zu wählen, um gegebenenfalls nur das ‘+' und nicht das ganze Geld zu verlieren. 
Kohärenz

Viertens: Ich gehe von Fällen aus, in denen Überlegungen hinsichtlich Kohärenz keine Rolle spielen.

Eine mögliche und sinnvolle Strategie, mit Unvergleichbarkeit umzugehen, kann darin bestehen, diejenige Option zu wählen, die besser verträglich ist mit anderen Zielen, die man verfolgt. So ist es möglich, dass der eine Studiengang wesentlich mehr Freiheiten verspricht, zum Beispiel weniger Präsenzzeit verlangt, und deshalb kompatibler ist mit dem Ziel, nebenbei viele andere schöne Dinge zu machen.

Die Idee besteht nicht darin, dass eine alternative Option $z$ existiert, welche besser als beide unvergleichbaren Optionen $x$ und $y$ ist, sondern darin, dass entweder $x$ oder $y$ besser verträglich mit anderen Zielen ist, die man verfolgt. In diesem Fall sollte also nicht beliebig gewählt werden, weil die eine unvergleichbare Option 'besser ins Leben passt'.

Diese Strategie zum Umgang mit Unvergleichbarkeit, die ich als Ansatz maximaler Kohärenz bezeichne, könnte in verschiedener Hinsicht präzisiert werden. So liesse sich beispielsweise im folgenden Sinne eine Hierarchisierung einbauen: Kohärenz zwischen wichtigen Dingen ist wichtiger als Kohärenz zwischen unwichtigen Dingen. Die Kohärenz zwischen Studium und Beruf ist wichtiger als die Kohärenz zwischen Studium und einem bestimmten Hobby. Falls die unvergleichbare Option $x$ mit wichtigen anderen Zielen und die unvergleichbare Option $y$ mit unwichtigen anderen Zielen inkompatibel ist, sollte dementsprechend $x$ gewählt werden..${ }^{11}$ Die Inkohärenz grösserer Projekte (Weltreise, akademische Laufbahn etc.) führt nämlich auch auf einer tieferen Ebene (Reisebüro aufsuchen, Aufsätze schreiben etc.) zu mehr Inkohärenz. Das heisst, ein grösseres Ziel verlangt, dass man viele andere 'kleinere' Entscheidungen trifft. Wer eine Weltreise machen will, geht gegebenenfalls auch zu Globetrotter. Wer heute eine akademische Laufbahn einschlagen will, schreibt auch Aufsätze. 
Fünftens: Ich denke, dass zwischen Satisfizieren und einer beliebigen Wahl kein wesentlicher Unterschied existiert und man, anstatt beliebig zu wählen, auch Ersteres tun kann.

Tennenbaum argumentiert, dass sogenanntes Satisfizieren (satisficing) die adäquate Strategie darstellt, wenn es sich bei den Optionen um vage Ziele handelt, welche 'grösser' sind und sich nur über die Zeit hinweg realisieren lassen (Tennenbaum 2015). Viele Fälle von Unvergleichbarkeit fallen auch in diese Kategorie. Stellt dementsprechend nicht Satisfizieren den richtigen Ansatz dar, und was bedeutet es, dass Ziele vage sind? Satisfizieren meint vereinfachend, dass es rational sein kann, eine Option zu wählen, welche ausreichend gut ist. Die Vagheit von Zielen hingegen meint, dass zwar gewisse Mittel gewählt werden müssen, um die Ziele erreichen zu können, die Art und Weise, wie das geschehen muss, aber bis zu einem bestimmen Grad unbestimmt ist.

Tennenbaum geht es um ganz bestimmte Entscheidungssituationen. Man kann sich vorstellen, dass ein Akteur zwei grössere Projekte verfolgt: einen Marathon laufen und in einem Chor singen. In diesem Fall, so Tennenbaum, sollte man versuchen, beide Ziele zu erreichen. Damit meint er Folgendes: (i) ausreichend, aber nicht unbedingt maximal trainieren, um den Marathon laufen zu können, und (ii) ausreichend, aber nicht unbedingt maximal singen üben, um im Chor mitmachen zu können. Das heisst, dass es sich um Fälle handelt, in denen sich die Ziele gegenseitig überhaupt nicht ausschliessen. Das Satisfizieren bezieht sich bei Tennenbaum nämlich auf die Mittel. Diese Beispiele fallen dementsprechend in einem gewissen Sinne auch in die Kategorie, in der eine alternative Option $z$ existiert (sowohl singen als auch Marathon laufen), welche besser als die möglicherweise unvergleichbaren Optionen $x$ (Marathon) und $y$ (singen) ist. Es ist aber möglich, die Strategie des Satisfizierens auf die Ziele selbst anzuwenden (anders als bei Tennenbaum). Da die unvergleichbaren Optionen jedoch oft genügend gut sind, besteht zwischen 'Wähle eine Option, die genügend gut ist!' und 'Würfle!' kein wesentlicher Unterschied. In beiden Fällen kann man beliebig wählen.

Nach diesen Präzisierungen und Abgrenzungen geht es im nächsten Teil des Kapitels nun darum, möglich Argumente zu entwickeln, weshalb nicht beliebig gewählt werden darf. 
10.4 Gründe gegen das beliebige Wählen

Ich versuche die Argumente gegen das beliebige Wählen im Folgenden zuerst so 'stark' wie möglich zu machen und anschliessend zurückzuweisen.

Erstens erwähne ich den Einwand, dass der Ansatz unplausible Konsequenzen involviert. Zweitens betrachte ich die Entgegnung, dass man nicht beliebig wählen soll, weil die Entscheidung nicht rückgängig gemacht werden kann. Drittens erläutere ich den Vorbehalt, dass eine beliebige Wahl für andere nicht nachvollziehbar und sogar schädlich sein kann. Viertens diskutiere ich Changs Idee, dass ein Akteur im Falle unvergleichbarer Handlungsoptionen eine Festlegung (commitment) tätigen sollte. In diesem Zusammenhang diskutiere ich verschiedene Deutungen, wobei die plausibelste gemäss meinem Dafürhalten darin besteht, dass ein Akteur eine existenzielle Stellungnahme abgeben sollte. Fünftens betrachte ich das Argument, dass Unvergleichbarkeit deshalb nicht zufällig aufgelöst werden soll, weil Gründe existieren, die wir bislang nicht in Betracht gezogen haben und die möglicherweise den Ausschlag für die eine oder andere Option geben können.

Einwand 1 - Sammle so viele Erfahrungen wie möglich

Man mag einwenden, dass der Ansatz impliziert, dass das Beibehalten des Status quo immer falsch ist, weil man nicht weiss, wie sich die andere Option anfühlen wird. Und man kann argumentieren, dass es alternative Entscheidungsregeln gibt - zum Beispiel: wähle immer dasjenige, was Du weniger kennst -, die eine grössere Vielzahl von Erfahrungen mit sich bringen. Diese Kritik trifft aber nicht zu. Ich sage nicht, dass man so viel Erfahrungen wie möglich machen soll. Andere Faktoren können für eine Akteurin wesentlich wichtiger sein. Mein Ansatz sagt nur, dass eine Wahl ceteris paribus schlecht ist, wenn sich das Wohlergehen verschlechtert. Wohlergehen determiniert dabei nicht den Wert der Optionen. Das Gegenteil ist der Fall: Der Wert der Optionen bestimmt das Wohlergehen. Der Lösungsvorschlag beinhaltet denn auch nicht, dass man durch neue Erfahrungen den Wert einer Option verbessern kann (vgl. zum Beispiel Paul 2014 für eine solche Position). 
Einwand 2 - Sei vorsichtig

Man kann einwenden, dass man nicht beliebig wählen darf, weil die Entscheidung nicht rückgängig gemacht werden kann. Anders formuliert: Das Risiko von 'trial-and-error experience' ist zu hoch. Aber auch dieser Einwand trifft nicht zu. Besonders 'hart' ist es natürlich dann, wenn die Entscheidung nicht rückgängig gemacht werden kann. Ich denke zwar, dass wir in der Tendenz eher vergessen, dass Entscheidungen auch rückgängig gemacht werden können und sich mehr Entschlüsse korrigieren lassen, als wir gemeinhin annehmen. Einige wichtige Fälle von Unvergleichbarkeit sind aber sicherlich auch deshalb hart, weil für die Person in dem Sinne viel auf dem Spiel steht, als gewisse Konsequenzen nicht rückgängig gemacht werden können. Es ist ein Faktum unseres Lebens, dass wir die Folgen unserer Entscheidungen manchmal nicht ungeschehen machen können. Dies stellt aber keine Begründung zugunsten einer alternativen Strategie dar. In dieser Hinsicht besteht vielmehr eine starke Disanalogie zwischen Theorie und Praxis. Eine wissenschaftliche oder philosophische Theorie kann stets revidiert werden. Praktische Entscheidungen hingegen lassen sich manchmal nicht korrigieren. Inwiefern die Entscheidung zwischen unvergleichbaren Optionen 'richtig' ist, kann in diesen Fällen auch nie mit Sicherheit gesagt werden. Wir können manchmal schlicht nicht in Erfahrung bringen, ob eine andere Entscheidung besser gewesen wäre. Unser Wohlergehen wird durch viele unterschiedliche Dinge bestimmt. Man kann nach Amerika auswandern, das Wohlergehen verschlechtert sich wegen einer Krankheit, und trotzdem war es die richtige Entscheidung. Die Ceteris-paribus-Klausel, die ich bei der Entwicklung meines Ansatzes gebraucht habe, existiert in der Realität nicht. Aber wie gesagt: Das ist kein Argument gegen das beliebige Wählen.

Einwand 3 - Sei nett zu den anderen

Gemäss Lutz Wingert kann das beliebige Wählen andere negativ beeinflussen. ${ }^{12}$ Wingert würde natürlich nicht behaupten, dass nicht-beliebiges Wählen andere nicht negativ beeinflussen kann. Im

12 Dieser Einwand wurde im Rahmen des Workshops Eigene Gründe, der 2015 an der ETH Zürich stattfand, von Wingert mündlich erläutert. Ich versuche, Wingerts Idee im Folgenden zu präziseren und zu plausibilisieren. 
Hintergrund von Wingerts Einwand steht vielmehr die Idee, dass es ein möglicher Grund gegen das beliebige Wählen darstellt. Wir leben im Netz sozialer Kontakte und sollten Rücksicht auf das Wohlergehen der anderen nehmen.

Im Besonderen sollten wir Sorge tragen für das Wohlergehen derjenigen Menschen, mit denen wir persönliche Beziehungen pflegen. Dies bedeutet unter anderem, dass unsere Handlungen andere nicht unnötig negativ beeinflussen und grundsätzlich nachvollziehbar sein müssen (Wie würden die anderen reagieren?). Unvergleichbarkeit zufällig aufzulösen, so der Einwand, ist für andere nicht nachvollziehbar und somit nicht rechtfertigbar. Vor den Augen der anderen Teammitglieder eine Münze zu werfen und gegebenenfalls mit dem Hockeyspielen aufzuhören, wäre nicht sehr umsichtig und könnte das Wohlergehen anderer beeinträchtigen. Und ebenso kann das picking die Teammitglieder 'vor den Kopf stossen', zum Beispiel wenn man auf die Frage, weshalb man mit Hockey aufhört, antwortet: 'Weil der FC Bayern verloren hat.'

Wir können Wingerts Gedanken als sogenanntes Argument der Rücksichtnahme wie folgt rekonstruieren:

1. Wenn man beliebig wählt, dann beeinflussen wir andere negativ.

2. Man darf andere nicht negativ beeinflussen.

$\therefore \quad$ Man darf nicht beliebig wählen.

Die erste Prämisse stellt eine empirische Behauptung dar, die ich Wingert zugestehe. Ich denke, dass sie nicht unplausibel ist. Die anderen Personen fühlen sich vielleicht in einer gewissen Hinsicht nicht respektiert, weil Würfeln so etwas wie Gleichgültigkeit signalisiert. Das heisst, dass das picking oder Würfeln beim Gegenüber als 'mir ist es egal' interpretiert werden kann (was de facto gar nicht der Fall sein muss).

Die zweite Prämisse stellt eine normative Behauptung dar, die wir so deuten können, dass es ceteris paribus moralisch schlecht ist, andere negativ zu beeinflussen. Oder anders formuliert: Es existiert ein moralischer Pro-tanto-Grund, andere nicht zu beeinträchtigen.

Nun verhält es sich natürlich so, dass sich andere Menschen von allem Möglichen negativ beeinflusst fühlen können, zum Beispiel von 
roten Socken. Es kann also nicht um Folgendes gehen: 'Ich fühle mich durch das Würfeln beeinträchtigt.' Vielmehr muss es sich um Nachstehendes drehen: 'Ich fühle mich durch das Würfeln gerechtfertigterweise beeinträchtigt.' Und wann ist dies gerechtfertigt? Eben genau dann, wenn das Würfeln nicht angebracht ist. In diesem Sinne stellt obiges Argument beziehungsweise Wingerts Überlegung eine petitio principii dar: Es wird vorausgesetzt, was gezeigt werden sollte.

Gemäss meinem Dafürhalten sollten wir jedoch versuchen, andere nicht negativ zu beeinflussen, unabhängig davon, ob es gerechtfertigt ist oder nicht. Wenn der Arbeitskollege keine roten Socken mag und man auch grüne besitzt, trägt man diese. In obigem Argument sind es Gründe der Rücksichtnahme, weshalb wir nicht würfeln dürfen.

Was folgt nun für das beliebige Wählen? Es resultiert, dass Wingerts Idee oder meine argumentative Rekonstruktion sicherlich nicht zeigt, dass das beliebige Wählen intrinsisch nicht gut ist. Das Argument kann nicht zeigen, dass am Würfeln selbst etwas schlecht ist. Das heisst wohlgemerkt nicht, dass Wingert nicht etwas Wichtiges im Blick hat, wobei ich dies ebenso verstehen würde, nämlich dass Rücksichtnahme wichtig ist.

Es kann also bedeuten, dass man den Teammitgliedern in höflicher Form verständlich macht, dass auch nach vertiefter Deliberation keine Evaluation möglich war, und deshalb beliebig gewählt wurde.

Oder es kann bedeuten, dass man im Geheimen würfelt und verschweigt, wie die Entscheidung zustande gekommen ist. Manchmal kann es ja auch aus reinen Gründen der Klugheit und nicht aus Rücksichtnahme von Vorteil sein, die Art und Weise, wie eine Entscheidung zustande gekommen ist, für sich zu behalten. Wenn zwischen zwei Stellenangeboten keine Evaluation möglich ist, in dem Sinne, dass keine Option besser ist, und man dennoch die eine Stelle wählt, kann es von Vorteil sein, dem neuen Arbeitgeber zu verschweigen, dass beliebig gewählt wurde. Stattdessen kann es sinnvoller sein - falls man sich überhaupt erklären muss -, auf die Gründe zu verweisen, welche für die angenommen Stelle sprechen, was die Argumente, welche für die andere Stelle sprechen, ja nicht zum Verschwinden bringt. 
Einwand 4 - Bekenne dich

Im Zentrum von Changs Überlegungen steht der Begriff des commitment, den wir als Festlegung oder im Kontext ihrer Arbeiten auch als Bekenntnis übersetzen können. Chang denkt, dass ein Akteur zwischen unvergleichbaren Optionen nicht würfeln darf, sondern ein Bekenntnis ablegen sollte. Chang führt meiner Ansicht nach ein psychologisches Argument ein, weshalb wir nicht beliebig wählen sollen.

Bevor ich versuchen werde, diesen Ansatz einerseits zu kritisieren, anderseits aber auch zu plausibilisieren, muss etwas ausgeholt werden.

Grundsätzlich kann man sich fragen, weshalb ein Akteur in der Lage sein soll, einen Entscheidungskonflikt just in dem Moment aufzulösen, in dem er wählt. Das Dilemma in Sophie's Choice beispielsweise - so der Titel eines Romans von William Styron, der ein in der Moralphilosophie diskutiertes Dilemma schildert - scheint durch Sophies Entscheidung nicht aufgelöst zu werden. Deshalb nicht, weil durch den Akt des Entscheidens keine neuen Gründe generiert werden können. Dies scheint auch auf Unvergleichbarkeit zuzutreffen. Oder anders formuliert: Eine Alternative kann durch den Akt des Entscheidens nicht besser oder schlechter gemacht werden.

Gemäss Chang, so habe ich sie verstanden, ist dies allerdings falsch. Chang geht davon aus, dass dasjenige, was sie als 'Wille' bezeichnet, eine entscheidende Rolle einnimmt, wenn in harten Fällen diejenigen Gründe, über die wir nachdenken, weder den Ausschlag für die eine noch die andere Option geben (Chang 2002b: 170). Damit befindet sie sich in guter Gesellschaft. Dass der 'Wille' im Falle von Unvergleichbarkeit eine bedeutende Rolle spielt, meint auch Raz (Raz 1997). Und Guckes sieht in Situationen, in denen sich die Gründe die Waage halten, sogar die Möglichkeit, 'frei’ entscheiden zu können, was mir nicht abwegig zu sein scheint (Guckes 2004). Anders als Guckes, sofern ich es richtig deute, denkt Chang jedoch, dass eine Alternative durch das Wählen besser gemacht werden kann. Was ist damit gemeint?

Changs Überlegung sollte nicht als empirischer oder phänomenologischer Punkt gedeutet werden. Wir besitzen eine gewisse Tendenz, schwierige Entscheidungen im Nachhinein zu rationalisieren. Damit meine ich, dass wir uns gerade bei schwierigen Entschlüssen oft 
selbst eine Geschichte erzählen, weshalb es besser war, dass wir so und nicht anders entschieden haben. Wenn man sich zwischen Bern und Basel nicht entscheiden kann und trotzdem nach Basel zieht, legt man es sich ex post irgendwie zurecht, dass Basel der bessere Wohnort ist.

Ich deute Chang dahingehend - und jetzt kommt das commitment ins Spiel -, dass 'Willen' eine solche normative Festlegung meint. Was muss darunter verstanden werden, und inwiefern kann damit eine Option besser gemacht werden?

Unter einer Festlegung werden sehr unterschiedliche Dinge begriffen. Peter und Schmid notieren: 'The clearest case of action from commitment is when one feels compelled to intervene in a certain matter, even if doing so leaves one worse off.' (Peter and Schmid 2007: 4). Gemäss diesem Verständnis meint eine Festlegung, dass wir etwas tun, weil wir denken, dass es richtig oder falsch ist. Bei Kant bedeutet es denn auch, dass man gemäss dem kategorischen Imperativ handelt (vgl. Pauer-Studer 2007: 74). Wie aber wird Festlegung von Chang verstanden? Am genausten setzt sie sich damit in ihrem Aufsatz 'Commitments, Reasons, and the Will' auseinander (Chang 2013b).

Eine Festlegung weist gemäss Chang vier charakteristische Merkmale auf: (i) wir können uns dazu entscheiden, uns festzulegen, (ii) es kann sich um einen andauernden Zustand oder ein konkretes Ereignis handeln, (iii) es ist etwas Individuelles (man muss nicht), (iv) dadurch werden neue Gründe generiert.

Wenn eine Festlegung als normative Überzeugung, Wunsch, Identifikation mit einem Wunsch, Absicht, Entscheidung, Plan oder Strategie verstanden wird, so Chang, können obige charakteristische Merkmale nicht erklärt werden. Nach Chang muss eine Festlegung vielmehr wie folgt gedeutet werden: 'Commitments are by their nature willings of some kind.' (Chang 2013b: 92). Dies wiederum versteht sie als 'willing something to be a reason' (Chang 2013b: 93), was wie folgt verstanden werden muss: 'Willing something to be a reason is the activity of placing your will - your very agency - behind its being a reason.' (Chang 2013b: 93).

Ein Blick nach Hollywood kann helfen, Changs Position besser zu verstehen: Wenn Oliver (Ashton Kutcher) im Film A Lot Like Love (So was wie Liebe) entscheidet, dass Emily (Amanda Peet) für ihn die Eine ist, dann legt er ein solches Bekenntnis ab. 
Nun können philosophische Thesen natürlich anhand von Filmen entwickelt werden, aber es stellt sicherlich kein Argument dar, dass etwas in einem Hollywood-Film vorkommt. Wie kann Changs Ansatz argumentativ plausibilisiert werden?

Klar ist: Für unwichtige Fälle von Unvergleichbarkeit, die keine neuen Optionen ermöglichen respektive unmöglich machen, wie etwa die Entscheidung, ob man das gesunde und weniger leckere oder ungesunde und leckere Menü bestellt, ist es sicherlich abwegig davon auszugehen, dass man sich zu einem Menü bekennt. Changs Ansicht stellt meiner Meinung nach eine mögliche Strategie dar, wie wichtige Fälle von Unvergleichbarkeit aufgelöst werden können. Ich denke, dass Chang in diesem Punkt zustimmen würde, da sie davon ausgeht - und das ist den meisten Fällen sicherlich richtig -, dass wir uns nicht zu Gegenständen, sondern zu Personen oder Projekten bekennen (Chang 2013b: 76). ${ }^{13}$

Inwiefern wir uns tatsächlich aktiv dazu entscheiden können, uns auf ein Projekt oder eine Person festzulegen, scheint gar nicht so klar zu sein. Oftmals scheint dies mit zunehmender Wertschätzung auch einfach zu passieren. Ich möchte Chang dies aber zugestehen und fokussiere auf den Aspekt der Gründe.

Was spricht für die Kreation neuer Gründe? Bei wichtigen Entscheidungen, zum Beispiel, ob Oliver Emily heiraten will, können durch die Heirat in dem Sinne neue Gründe generiert werden, als Oliver 'alles' versuchen sollte, um mit Emily zusammenzubleiben oder sie so gut wie möglich zu unterstützen. Dies ist durchaus möglich. Es passiert jedoch ex post. Im Falle von Unvergleichbarkeit sollte durch eine Festlegung eine Option ex ante besser gemacht werden können. Die Frage, welche uns im Falle von Unvergleichbarkeit beschäftigt, ist vielmehr, weshalb Oliver Emily und nicht jemand anderen heiraten will. Uns interessieren Fälle, in denen ein Akteur keine eindeutige Evaluation vollziehen kann. Im Film A Lot Like Love ist dies offensichtlich nicht der Fall. Oliver ist verrückt nach Emily. Wenn man sich allerdings zu einer Person auf diese Weise bekennen kann, damit die Interessen der anderen Person zu eigenen Gründen macht, scheint

13 Die These, dass in allen harten Fällen nicht beliebig gewählt werden soll, kann meines Erachtens folglich von Chang nicht konsistent vertreten werden, weil ihre normative Begründung unwichtige harte Fälle (Kaffee oder Tee?) nicht einschliesst. 
dies überhaupt kein Fall von Unvergleichbarkeit mehr zu sein. Nicht nur besteht also die Gefahr darin, dass Changs Idee der Festlegung nicht mit dem Phänomen der Unvergleichbarkeit kompatibel ist, auch ist die These fraglich, dass durch eine Festlegung ex ante ein zusätzlicher Grund geschaffen und somit eine unvergleichbare Option verbessert werden kann. In diesem Zusammenhang wird manchmal der technische Ausdruck bootstrapping verwendet. Im Falle der theoretischen Vernunft ist damit gemeint, dass eine Überzeugung, zum Beispiel, dass Gott existiert, keinen zusätzlichen Grund für die Existenz Gottes liefert: 'You cannot, by means of your beliefs, bootstrap a new reason into existence, to add the evidence.' (Broome 2013: 82). Im Falle der praktischen Vernunft ist damit gemeint, dass eine Absicht, eine Entscheidung oder eben auch eine Festlegung keinen zusätzlichen Grund liefert, etwas zu tun. In seinem Aufsatz 'Rational Self-Commitment' schreibt Verbeek: 'Bootstrapping seems to spell problems for the feasibility of rational commitment. If decisions do not create reasons for a course of action, decisions do not rationalize the future action.' (Verbeek 2007: 161). Verbeek vertritt die Meinung, dass Absichten neue Gründe generieren können ('intentions bootstrap reason for action into existence'). Die Reihenfolge scheint aber eher umgekehrt zu sein: ' $x$ ist besser, und deshalb kann ich mich zu $x$ und nicht zu $y$ bekennen.' Wenn Oliver Emily liebt, kann er sich zu ihr bekennen (dies stellt aber keinen Fall von Unvergleichbarkeit dar).

Ich plädiere deshalb dafür, die Idee, dass im Falle von Unvergleichbarkeit ein Bekenntnis getätigt werden sollte, nicht als Kreation neuer Gründe, sondern als existenzielle Stellungnahme zu verstehen. Was ist gemeint? Die Gründe, die für die unvergleichbaren Optionen sprechen, sind in der Regel verschiedenartig. Der eine Grund kann zum Beispiel den Wert eines abenteuerlichen und der andere Grund den Wert eines geregelten Lebens zum Ausdruck bringen. Eine existenzielle Stellungnahme meint: Entweder bekennt man sich zum Abenteuer oder zu einem geregelten Leben.

Man könnte sagen, dass deshalb eine existenzielle Stellungnahme verlangt wird, weil es auch entscheidend für die eigene Identität ist, wie man sich entscheidet. Handelt es sich nämlich um eine wichtige Entscheidung, die viele andere Optionen öffnet respektive schliesst und längere Zeiträume betrifft, die den Akteur selbst unter- 
schiedlich prägen, ist die Entscheidung auch relevant für die Identität der jeweiligen Person. Je nachdem, wie man entscheidet, ist dann nicht nur die 'Welt', sondern auch man selbst anders. Es geht hierbei nicht um die Frage, wer man ist, sondern darum, wer man sein will. Und dies sollte eben nicht durch einen Würfel, sondern ein existenzielles Bekenntnis erfolgen ('Ich bin der Abenteuer-Typ').

Was ist davon zu halten? Es mag sein, dass es psychologisch betrachtet ein Problem darstellt, sich mit einer beliebigen Wahl zu identifizieren, da man dann nicht in einem emphatischen Sinne hinter der Entscheidung steht. Damit meine ich, dass man das Ergebnis des Würfelns vielleicht nicht akzeptieren oder glauben kann. In diesem Punkt liegt Chang meines Erachtens richtig. So kann es nur schwerlich annehmbar sein, wegen des FC Bayerns das Hockeyspielen zu beenden. In dieser Hinsicht, so könnte man sagen, muss man sich überlisten und, anstatt beliebig zu wählen, vielmehr eine Geschichte erzählen, weshalb man sich so und nicht anders entscheiden will. Das ist jedoch kein normatives Argument. Es scheint sich vielmehr um eine psychologische Strategie beziehungsweise eine Rationalisierung zu handeln.

Aber existieren tatsächlich keine rationalen Argumente, die Changs Position stützen? Man könnte argumentieren, dass ihr Ansatz kompatibel mit der Idee von Autonomie ist.

Spricht das Konzept der Autonomie dafür, die existenzielle Festlegung der beliebigen Wahl vorzuziehen? Der Verweis auf Autonomie ist oft Rhetorik. Aber das heisst nicht, dass man die Konzeption nicht explizieren kann. Und wenn wir das machen, scheint es eine offene Frage zu sein, in welchem Verhältnis Autonomie und beliebiges Wählen zueinander stehen. Es bieten sich verschiedene Möglichkeiten an, dieser Frage nachzugehen. Einerseits kann sie auf der Ebene verschiedener Autonomiekonzeptionen diskutiert werden: Mit welcher Konzeption von Autonomie ist beliebiges Wählen kompatibel und mit welcher nicht? Anderseits kann sie auf der Ebene verschiedener Intuitionen diskutiert werden, die der Verwendung des Begriffs der Autonomie unterliegen: Ist das beliebige Wählen mit diesen Intuitionen kompatibel oder nicht?

Bleiben wir bei den Intuitionen. Gemäss Betzler sind es zwei zentrale Intuitionen, die der Verwendung des Begriffs der Autonomie 
zugrunde liegen: Kontrolle und Treue zu sich selbst (Betzler 2013: 12). Ist das beliebige Wählen damit kompatibel oder nicht? Es kommt darauf an, was darunter verstanden wird. Wenn Kontrolle meint, dass man den Ausgang der Entscheidung kontrolliert, dann ist es nicht kompatibel mit dem beliebigen Wählen. Wenn Kontrolle meint, dass man die Mittel kontrolliert, wie die Entscheidung zustande kommt, dann ist es kompatibel mit dem beliebigen Wählen. Wenn Treue zu sich selbst inhaltlich neutral verstanden wird, dann ist es kompatibel mit dem beliebigen Wählen. Wenn Treue zu sich selbst nicht inhaltsneutral verstanden wird, dann ist es unter Umständen nicht kompatibel mit dem beliebigen Wählen. In diesem Fall könnte man argumentieren, dass man gewisse Dinge wie das beliebige Wählen schlicht nicht authentisch wollen darf. Weil es allerdings nicht darum gehen kann, dass das authentische Würfeln per se unmöglich ist - man frage einen gambler - sollte hierbei eher argumentiert werden, dass das beliebige Wählen in gewissen Lebensbereichen oder Entscheidungssituationen nicht authentisch gewollt werden darf.

Es ist klar, dass ein Münzwurf im Falle von Vergleichbarkeit keine geeignete Strategie ist, um eine Entscheidungssituation aufzulösen. Wenn $x$ besser als $y$ ist, sollte nicht aufgrund des FC Bayern $y$ gewählt werden (ausser, einem ist alles egal). Wenn aber die existenzielle Stellungnahme nicht auf einer Evaluation basiert - es ist besser das Abenteuer zu suchen -, dann ist unklar, weshalb nicht auch beliebig gewählt werden darf.

\section{Einwand 5 - Suche weiter}

Gemäss einem weiteren potenziellen Einwand sollten wir nicht in uns selbst hineinblicken und uns zu etwas bekennen, sondern 'in der Welt' nach neuen Gründen suchen, um eine begründete Entscheidung treffen zu können. Diesem Argument zufolge soll Unvergleichbarkeit deshalb nicht zufällig aufgelöst werden, weil Gründe existieren, die wir bislang nicht in Betracht gezogen haben und die möglicherweise den Ausschlag für die eine oder die andere Option geben könnten. Gemäss diesem Ansatz haben wir nur einige, aber niemals alle für die Entscheidungssituation relevanten Gründe gesehen. Dieser Ansatz würde denn auch eine Erklärung dafür liefern, warum es im Falle von 
Unvergleichbarkeit nicht abwegig scheint, mit der Deliberation fortzufahren und zu überlegen, was man tun will. In solchen Fällen ist dies sinnvoll, weil man unter Umständen noch nicht alle für die Entscheidungssituation relevanten Gründe und Werte erfasst hat.

Ich denke, dass dieser Ansatz die alltägliche Phänomenologie im folgenden Sinne relativ gut abbildet: Wir würfeln de facto beinahe nie. Dies könnte damit zu tun haben, dass Unvergleichbarkeit ein absolutes Randphänomen darstellt. Oder es könnte damit zu tun haben, dass Unvergleichbarkeit in dieser Arbeit zu idealisiert dargestellt worden ist. Ich denke jedoch, dass es vielmehr damit zusammenhängt, dass wir im Falle unvergleichbarer Handlungsoptionen weitersuchen und dann oft aufgrund irgendeiner neuen Gegebenheit entscheiden.

Aber es geht nicht darum, was wir im Falle von Unvergleichbarkeit tun, sondern, was wir gerechtfertigterweise tun. Das Argument der neuen Gründe steht und fällt mit der Annahme, dass Gründe nie ausgehen. Die Erwiderung auf dieses Argument ist dieselbe, wie wenn jemand behauptet, dass Unvergleichbarkeit stets faktische Unsicherheit beinhaltet (vgl. Kapitel 3). Dagegen spricht, dass man manchmal genügend sicher ist, alle für den Entscheid relevanten Werte erfasst zu haben, und man die Handlungsoptionen aufgrund von bereits in Betracht gezogenen Faktoren dennoch nicht als besser/schlechter/ gleich gut einstufen kann. Das Weitersuchen oder das Wählen aufgrund einer neuen Gegebenheit wie etwa des Ratschlags eines Freundes ist dann nicht weniger zufällig respektive mehr gerechtfertigt als das beliebige Wählen. Und handelt es sich bei der neuen Gegebenheit tatsächlich um einen normativen Grund, der den Ausschlag geben kann - der Freund einem zum Beispiel 'die Augen öffnet' -, dann war die Unvergleichbarkeit nur scheinbar.

\subsection{Zusammenfassung}

Erstens wurde das Konzept einer beliebigen Wahl eingeführt. Zweitens wurde das Argument erläutert, weshalb beliebig gewählt werden darf. Drittens wurde gezeigt, wann beliebig gewählt werden darf. Viertens wurden Einwände zurückgewiesen, weshalb nicht beliebig gewählt werden darf. 
Wir können deshalb Folgendes festhalten: Im letzten Teil des Buches wurde ein Argument dafür genannt, dass der Art und Weise, wie man wählt, keine entscheidende Bedeutung zukommt. Deshalb nicht, weil es Erfahrung braucht, die Güte unvergleichbarer Optionen beurteilen zu können. Dementsprechend ist weniger die Rede davon, dass ein Akteur dazu aufgefordert ist, in bestimmter Weise zu wählen, nach neuen Gründen zu suchen oder sich zu bekennen, als vielmehr im Nachhinein in bestimmter Weise zu reagieren. Die Erfahrung selbst erfolgt ja erst nach der Entscheidung. Dies legt den Schluss nahe, dass in der Tendenz - ich will nicht behaupten, dass keine Ausnahmen existieren - die Entscheidung zwischen unvergleichbaren Optionen nicht zu lange hinausgezögert werden sollte. Man sagt manchmal, dass Ambivalenzen in der eigenen Persönlichkeit ausgehalten werden sollen respektive müssen. Im Falle unvergleichbarer Optionen, so meine ich, ist die adäquate Strategie eher, in die 'Offensive' zu gehen, Erfahrung zu sammeln, Dinge gegebenenfalls wieder zu korrigieren und, falls dies nicht möglich ist, sich zu arrangieren. Hierbei kann es sicherlich helfen, wenn man ex post die Dinge ausblendet, die für die nicht-gewählte Option sprachen, und versucht, die gewählte Option durch neue Entscheidungen zu verbessern. 
Rückblick 
Als Erstes stand die Frage im Mittelpunkt, was Unvergleichbarkeit ist. Ich habe eine Präferenzversion von Unvergleichbarkeit eingeführt, verschiedene Typen von Unvergleichbarkeit unterschieden, die auf einen Wertkonflikt (evaluatives Unwissen) zurückgeführt werden können, und Unvergleichbarkeit von benachbarten Phänomenen wie Gleichgültigkeit abgegrenzt. Die grundlegende Konsequenz, die aus den ersten fünf Kapiteln gefolgert werden kann, ist, dass Unvergleichbarkeit ein reales Phänomen ist.

Danach wurde der Frage nachgegangen, worin das Problem für die Rationalität besteht. Ich habe argumentiert, dass Vergleichbarkeit sehr problematische Komponenten besitzt, aber unter bestimmten Bedingungen als Voraussetzung einer rationalen Wahl angesehen werden kann. Optionen sind genau dann vergleichbar, wenn sie entweder besser oder schlechter als oder gleich gut wie die jeweilig andere Option sind, wobei im Falle von Unvergleichbarkeit die 'Lücke' zwischen den möglichen und gewählten Alternativen nicht mit einer Präferenz respektive Werten geschlossen werden kann. Weil diese Art der Evaluation versagt, lassen sich die Optionen in keine begründete Ordnung bringen. Dabei habe ich belegt, dass bisherige Versuche zur Lösung des Problems der Unvergleichbarkeit entweder nicht angemessen beschreiben oder nicht hinreichend zeigen, wie harte Fälle aufgelöst werden können. Die grundlegende Konsequenz, die aus dem sechsten, siebten, achten und neunten Kapitel gefolgert werden kann, ist, dass eine Akteurin zwischen unvergleichbaren Optionen oft nicht rational entscheiden kann.

Im letzten Teil bin ich zum Schluss gelangt - ausgehend von einem bestimmten Konzept von Wohlergehen -, dass eine Akteurin beliebig wählen darf und es unter bestimmten Bedingungen - faktische Unwissenheit ist vernachlässigbar, Kohärenz spielt keine Rolle u. a. - keine Rolle spielt, was man tut. Diese These ist nicht unbedingt intuitiv, aber ich denke, dass ich sie gut begründet habe. Die Ideen, wie mit Unvergleichbarkeit umgegangen werden kann, stellen einen Versuch dar, einen Aspekt von Unvergleichbarkeit zu erschliessen, der bislang nicht oder beinahe nicht untersucht worden ist.

Insgesamt bringt dieses Buch nicht nur die akademische Debatte in verschiedener Hinsicht voran, sondern eröffnet auch eine für unseren Alltag relevante Perspektive, wie über Unvergleichbarkeit nachgedacht werden kann. 


\section{Literaturverzeichnis}

A Adler, Matthew (1998): 'Law and Incommensurability', in: University of Pennsylvania Law Review 146, 1169-1184

Aldred, Jonathan (2013): 'Justifying precautionary policies: Incommensurability and uncertainty', in Ecological Economics 96, 132-140

Anderson, Elizabeth (1997): 'Practical Reason and Incommensurable Goods', in: Incommensurability, Incomparability, and Practical Reason, R. Chang (Hg.), Cambridge: Harvard University Press

Andreou, Chrisoula (2005): 'Incommensurable Alternatives and Rational Choice', in: Ratio XVIII, 249-361

Andreou, Chrisoula (2011): 'Choosing Well: Value Pluralism and Patterns of Choice', in: New Waves in Ethics, T. Brooks (Hg.), Basingstoke: Palgrave Macmillan

Andreou, Chrisoula (2015): 'Parity, Comparability, and Choice', in: The Journal of Philosophy CXII, 5-22

Anscombe, G. E. M. (1978): Absicht, Berlin: Suhrkamp

Aristoteles (2008): Nikomachische Ethik, Ursula Wolf(Hg.), Reinbek bei Hamburg: Rowohlt Taschenbuch

Aristotle (1984): The complete work of Aristotle, Princeton: Princeton University Press

Arpaly, Nomy (2000): 'On Acting Rationally Against One’s Own Best Judgement', in: Ethics 110, 448-513

Audi, Robert (1990): 'Weakness of Will and Rational Action', in: Australian Journal of Philosophy 68, 270-281

Aumann, Robert (1962): 'Utility Theory without the Completeness Axiom', in: Econometrica 30, 445-462

B Badhwar, Neera (2014): Well-Being, New York: Oxford University Press Baggini, Julian und Fosl, Peter (2010): The Philosopher's Toolkit, Oxford: Blackwell Publishing Baumann, Peter und Betzler, Monika (2004): Practical Conflicts, Cambridge: Cambridge University Press

Beisbart, Claus (2004): Handeln begründen, Berlin: Lit Verlag Berlin, Isahiah (1969): Four Essays on Liberty, Oxford: Oxford University Press 
Betzler, Monika (1999): 'Praktische Überlegung und Wertinkommensurabilität', in: Die Zukunft des Wissens. Proceedings of the 18th Congress of the German Philosophical Association, Jürgen Mittelstrass (Hg.), Konstanz: Universitätsverlag

Betzler, Monika (2013): 'Einleitung: Begriff, Konzeption und Kontexte der Autonomie', in: Autonomie der Person, M. Betzler (Hg.), Münster: mentis

Boot, Martjin (2007): Incommensurability, Incomplete Comparability and the Scales of Justice, Dissertation (Universität Oxford)

Boot, Martijn (2009): 'Parity, Incomparability and Rationally Justified Choice', in: Philosophical Studies 146, 75-92

Bratman, Michael (1987): Intention, Plans, and Practical Reason, Cambridge: Harvard University Press

Breyer, Friedrich (2007): Mikroökonomik, Berlin: Springer

Broome, John (1991): Weighing goods: equality, uncertainty and time, Oxford: Basil Blackwell

Broome, John (1997): 'Is Incommensurability Vagueness?', in: Incommensurability, Incomparability, and Practical Reason, R. Chang (Hg.), Cambridge: Harvard University Press

Broome, John (2004): Weighing Lives, Oxford: Oxford University Press Broome, John (2013): Rationality Trough Reasoning, Oxford: Wiley Blackwell

Brun, Georg und Hirsch Hadorn, Gertrude (2009): Textanalyse in den Wissenschaften, Zürich: vdf Hochschulverlag an der ETH

Buchanan, Allen (1988): Efficiency, and the market, Totowa (N.J.): Rowman Littlefield

Byron, Michael (2000): Satisficing and Maximizing, Cambridge: Cambridge University Press

C Carlson, Erik (2004): 'Broome’s Argument against Value Incomparability', in: Utilitas 16, 220-224

Carlson, Erik (2010): 'Parity Demystified', in: Theoria 76, 119-128

Carlson, Erik (2011): 'The Small-Improvement Argument Rescued', in: The Philosophical Quarterly 61, 171-174

Carnap, Rudolf (1959): Induktive Logik und Wahrscheinlichkeit, bearbeitet von W. Stegmüller, Wien: Springer 
Chang, Ruth (1997): 'Introduction', in: Incommensurability, Incompara-bility, and Practical Reason, R. Chang (Hg.), Cambridge: Harvard Uni-versity Press Chang, Ruth (2001): 'Against Constitutive Incommensurability or Buying and Selling Friends', in: Noûs 35, 33-60

Chang, Ruth (2002a): 'The Possibility of Parity', in: Ethics 112, 659-688 Chang Ruth (2002b): Making Comparisons Count, New York: Routledge Chang, Ruth (2004): 'All Things Considered', in: Philosophical Perspec-tive 18, 1-22 Chang, Ruth (2005): 'Parity, Internal Value, and Choice', in: Ethics 115, 331-350

Chang, Ruth (2009): 'Voluntarist Reasons and the Sources of Normativity', in: Reasons for Action, D. Sobel und S. Wall (Hg.), New York: Cambridge University Press Chang, Ruth (2012): 'Are Hard Choices Cases of Incomparability?', in: Philosophical Issues 22, 106-126

Chang, Ruth (2013a):

$c s$, H. LaFollette (Hg.), Oxford: Blackwell

Chang, Ruth (2013b): 'Commitment, Reasons, and the Will', in: Oxford Studies in Metaethics 8, 74-113

Chang, Ruth (2014): 'Value Incomparability and Incommensurability' in: Oxford Handbook of Value Theory, I. Hirose und J. Olson (Hg.), Ox-ford: Oxford University Press Chang, Ruth (2015a): 'Comparativism: The Ground of Rational Choice', in: Weighing Reasons, E. Lord und B. McGuire (Hg.), Oxford: Oxford University Press

Chang, Ruth (2015b): 'Value Pluralism', in: International Encyclopedia of the Social \& Behavioral Sciences 25, J. Wright (Hg.), Oxford: Elsevier Chang, Ruth: 'The existentialist of hard choices' (Interview von Richard Marshall mit Ruth Chang) http://www.3ammagazine.com/3am/theex-istentialist-of-hard-choices/ (Erstveröffentlichung 4. April 2014) Constantinescu, Christian (2012): 'Value incomparability and indeterminacy', in: Ethical Theory and Moral Practice 15, 57-70

Cubitt, Robin und Sugden, Robert (2001): 'On Money Pumps', in: Games and Economic Behaviour 37, 121-160 
D D’Agostino, Fred (2003): Incommensurability and Commensuration, Hampshire: Ashgate

D’Agostino, Fred (2014): ‘Verballed? Incommensurability 50 years on', in: Synthese 191, 517-538

Daly, Chris (2010): An Introduction to Philosophical Methods, Toronto: Broadview Press

Davidson, Donald (1963): 'Actions, reasons and causes', in: Journal of Philosophy 60, 685-700

De Jonge, Jan (2012): Rethinking Rational Choice Theory, Basingstoke: Palgrave Macmillan

De Sousa, Ronald (1974): ‘The good and the True', in: Mind 83, 534-551

Dworkin, Ronald (1977): Taking Rights Seriously, Cambridge: Harvard University Press

E Elson, Luke (2014): 'Heaps and Chains: Is the Chaining Argument for Parity a Sorites?', in: Ethics 124, 557-571

Espinoza, Nicolas (2008): 'The Small Improvement Argument', in: Synthese 165, 127-139

- $\quad$ Elster, John (1984): Ulysses and the Sirens. Studies in Rationality and Irrationality, Cambridge: Cambridge University Press

F Feyerabend, Paul (1997): Philosophy, science and society, Cambridge: Polity Press

Fröding, Barbro und Peterson, Martin (2012): 'Virtuous Choice and Parity', in: Ethical Theory and Moral Practice 15, 71-82

G Gert, Joshua (2004): 'Value and Parity', in: Ethics 114, 492-510 Glock, Hans-Johann (2008): What is analytic philosophy?, Cambridge: Cambridge University Press

Greimann, Dirk (2007): 'Regeln für das korrekte Explizieren von Begriffen', in: Zeitschrift für philosophische Forschung 61, 261-282 Griffin, James (1996): Value judgement, Oxford: Clarendon Press Griffin, James (1997): 'Incommensurability: What's the Problem?', in: Incommensurability, Incomparability, and Practical Reason, R. Chang (Hg.), Cambridge: Harvard University Press

Grimm, Stephen (2007): 'Easy Cases and Value Incommensurability', in: Ratio XX, 26-44

Guckes, Barbara (2004): 'Do Conflicts make us free?', in: Practical Conflicts, P. Baumann und M. Betzler (Hg.), Cambridge: Cambridge University Press 
Gustafsson, Johan (2013): 'Indeterminacy and the Small-Improvement Argument', in: Utilitas 25, 433-445

Gustafsson, Johan und Espinoza, Nicolas (2010): 'Conflicting Reasons in the Small-Improvement Argument', in: The Philosophical Quarterly $60,754-763$

H Hahn, Susanne (2013): Rationalität, Münster: mentis Hare, Caspar (2010): 'Take the Sugar', in: Analysis 70, 237-247 Hare, Richard (1972): The Language of Morals, London: Oxford University Press

Hausman, Daniel (2012): Preference, value, choice, and welfare, Cambridge: Cambridge University Press

Henning, Tim (2015): 'From Choice to Chance - Saving People, Fairness, and Lotteries', in: Philosophical Review 124, 169-206

Hsieh, Nien-hê (2005): 'Equality, Clumpiness and Incomparability', in: Utilitas 17, 180-204

Hsieh, Nien-hê (2007): ‘Is Incomparability a Problem for Anyone?', in: Economics and Philosophy 23, 65-80

Hume, David (1986): A treatise of human nature, L. Selby-Digge (Hg.), Oxford: Oxford University Press

K Kavka, Gregory (1991): 'Is Individual Choice Less Problematic than Collective Choice?', in: Economics and Philosophy 7, 143-165

Keefe, Rosanna (2007): Theories of Vagueness, Cambridge: Cambridge University Press

Kierkegaard, Søren (1885): Entweder-Oder, Leipzig: Richter

Klocksiem, Justin (2010): 'In Defense of the Trichotomy Thesis', in: Acta Analytica 25, 317-327

Kornhauser, Levis (1998): 'No Best Answer?', in: University of Pennsylvania Law Review 146, 1599-1637

Korsgaard, Christine (1996): The Sources of Normativity, Cambridge: Cambridge University Press

Kuhn, Thomas (2012): The structure of scientific revolutions, 4. Ausgabe, Chicago: The University of Chicago Press

L Levi, Isaac (1986): Hard Choices, Cambridge: Cambridge University Press

Lichtenstein, Sarah und Slovic, Paul (1971): 'Reversal of Preferences between Bids and Choices in Gambling Decisions', in: Journal of Experimental Psychology 89, 46-55 
Lichtenstein, Sarah und Slovic, Paul (2006): 'The Construction of Preference: An Overview', in: The Construction of Preference, S. Lichtenstein und P. Slovic (Hg.), Cambridge: Cambridge University Press Lindman, Harold (1971): 'Inconsistent Preferences Among Gambles', in: Journal of Experimental Psychology 89, 390-397

- $\quad$ Lockhart, Ted (2000): Moral Uncertainty and its Consequences, Oxford: Oxford University Press

- $\quad$ Lukes, Steven (1997): 'Comparing the incomparable: Trade-offs and sacrifices', in: Incommensurability, Incomparability, and Practical Reason, R. Chang (Hg.), Cambridge: Harvard University Press

M MacAskill, William (2014): Normative Uncertainty, Dissertation (Universität Oxford)

Mas-Colell, Andreu, Whinston, Michael und Green, Jerry (1995): Microeconomic Theory, New York: Oxford University Press

Messerli, Michael und Reuter, Kevin (2017): 'Hard Cases of Comparison', in: Philosophical Studies 174, 2227-2250

Messerli, Michael und Reuter, Kevin (2019), 'Decisions Against Preferences', in: Proceedings of the 41st Annual Conference of the Cognitive Science Society, A. K. Goel, C. M Seifert und C. Freska (Hg.), Montreal, QB Cognitive Science Society, 2345-2350

- Messerli, Michael und Brunnabend, Daniel (Manuskript): 'The Chaining Argument'

- Messerli, Michael (Manuskript): 'Is it appropriate to randomly choose in hard choice scenarios?'

Messerli, Michael (Manuskript): 'Will I always imagine?'

Millgram, Elijah (1997): Practical Induction, Cambridge: Harvard University Press

Millgram, Elijah (2010): Praktische Induktion, Übersetzung von J. Schulte, Paderborn: mentis

- Morton, Adam (1991): Disasters and dilemmas, Cambridge: Basil Blackwell

N Nagel, Thomas (1979): 'The fragmentation of value', in: Mortal Questions, Cambridge: Cambridge University Press

Nida-Rümelin, Julian (1999): 'Was ist ein praktischer Grund?', in: Die Zukunft des Wissens. Proceedings of the 18th Congress of the German Philosophical Association, Jürgen Mittelstrass (Hg.), Konstanz: Universitätsverlag 
P Parfit, Derek (1986): Reasons and Persons, Oxford: Clarendon Press Pauer-Studer, Herlinde (2007): 'Instrumental Rationality versus Practical Reason: Desires, Ends, and Commitment', in: Rationality and Commitment, F. Peter und B. Schmid (Hg.), Oxford: Oxford University Press

Paul, Laurie Ann (2014): Transformative experience, Oxford: Oxford University Press

Paul, Laurie Ann (2015): 'What you can't expect when you're expecting', in: Res Philosophica 92, 1-23

Peter, Fabienne und Schmid, Bernhard (2007): 'Rational Fools, Rational Commitments', in: Rationality and Commitment, F. Peter und B. Schmid (Hg.), Oxford: Oxford University Press

Peterson, Martin (2007): 'Parity, Clumpiness and Rational Choice', in: Utilitas 19, 505-513

Pfister, Jonas (2013): Werkzeuge des Philosophierens, Stuttgart: Reclam Pinkowski, David (2013): 'Practically Equal: An Analysis of the Practical Nature of Equality and Incomparability', in: Acta Analytica 28, 457-470 Platon (1994): Sämtliche Werke, Band 3, Reinbek bei Hamburg: Rowohlt Verlag

R Rabinowicz, Wlodek (2005): 'Modeling Parity and Incomparability', in: Patterns of Value: Essays on Formal Axiology and Value Analysis 2, 201-228

Rabinowicz, Wlodek (2008): 'Value Relations', in: Theoria 74, 18-49

Rabinowicz, Wlodek (2009): 'Incommensurability and Vagueness', in: The Aristotelian Society (Supplementary Volume), 71-94

Rauschmayer, Felix (2001): 'Philosophical aspects of incommensurability and incomparability', in: Informatica 12, 119-132

Raz, Joseph (1978): Practical Reasoning, Oxford: Oxford University Press Raz, Joseph (1986): The Morality of Freedom, Oxford: Clarendon Raz, Joseph (1997): 'Incommensurability and Agency', in: Incommensurability, Incomparability, and Practical Reason, R. Chang (Hg.), Cambridge: Harvard University Press

Raz, Joseph (1999): Practical Reason and Norms, Oxford: Oxford University Press

Regan, Donald (1997): 'Value Comparability', in: Incommensurability, Incomparability, and Practical Reason, R. Chang (Hg.), Cambridge: Harvard University Press 
Resnik, Michael (1990): Choices: An Introduction to Decision Theory, Minneapolis: University of Minnesota Press

Reuter, Kevin und Messerli, Michael (2017): 'How Not to Characterise a Hard Choice', in: Ratio 30, 494-521

Reuter, Kevin und Messerli, Michael (2018): ‘Transformative Decisions', in: Journal of Philosophy 115, 313-335

Richardson, Henry (1994): Practical Reasoning about Final Ends, Cambridge: Cambridge University Press

Robinson, Richard (1950): Definitions, Oxford: Clarendon Press

Rott, Hans (2001): Change, choice and inference, Oxford: Clarendon Press

S Sartre, Jean-Paul (1957): 'Existentialism is a Humanism', in: Existentialism from Dostoevsky to Sartre, W. Kaufmann (Hg.), New York: Meridian Savage, Leonard (1954): The Foundations of Statistics, New York: John Wiley \& Sons Inc.

Scanlon, Thomas (1998): What we owe to each other, Cambridge: Harvard University Press

Scanlon, Thomas (2008): Moral Dimensions: Permissibility, Meaning, Blame, Cambridge: Belknap Press

Schaber, Peter (1994): 'Sind alle Werte vergleichbar? Kosten-NutzenAnalyse und das Inkommensurabilitätsproblem', in: Analyse und Kritik 2, 153-165

Schauer, Frederick (1998): 'Instrumental Commensurability', in: University of Pennsylvania Law Review 146, 1215-1233

Schick, Frederic (1984): Having Reasons, Princeton: Princeton University Press

Schmidtz, David (2000): 'Satisficing as a Humanly Rational Strategy', in: Satisficing and Maximizing, M. Byron (Hg.), Cambridge: Cambridge University Press

Seel, Gerhard (1977): 'Struktur und Geltung des wirtschaftlichen Handelns', in: Lehrstücke der praktischen Philosophie und der Ästhetik, K. Bärthlein und G. Wolandt (Hg.), Basel/Stuttgart: Schwabe Sellmaier, Stephan (2007): Langfristiges Entscheiden, Berlin: Lit Verlag Sen, Amartya (1986): 'Behaviour and the Concept of Preference', in: Rational Choice, J. Elster (Hg.), Oxford: Basil Blackwell

Sen, Amartya (1997): 'Maximization and the Act of Choice', in: Econometrica $65,745-779$ 
Sen, Amartya (2000): 'Consequential Evaluation and Practical Reasoning', in: The Journal of Philosophy 97, 477-502

Sen, Amartya (2004): 'Incompleteness and Reasoned Choice', in: Synthese 140, 43-59

Sepielli, Andrew (2009): 'What to Do When You Don't Know What to Do', in: Oxford Studies in Metaethics IV, Oxford: Oxford University Press, 5-28

Sepielli, Andrew (2012): 'Normative uncertainty for non-cognitivists', in: Philosophical Studies 160, 191-207

Shafir, Eldar, Simonson, Itamar und Tversky, Amos (2006): 'ReasonBased Choice' in: The Construction of Preference, S. Lichtenstein und P. Slovic (Hg.), Cambridge: Cambridge University Press

Simon, Herbert (1953): A behavioral model of rational choice, Santa Monica: Rand

Sinnott-Armstrong, Walter (1985): 'Moral dilemmas and Incomparability', in: American Philosophical Quarterly 22, 321-329

Sinnott-Armstrong, Walter (1988): Moral Dilemmas, Oxford: Basil Blackwell

Steglich-Petersen, Asbjørn (2012): 'Clumps and Pumps: Clumpiness, Resolution and Rational Choice', in: Utilitas 24, 118-125

Stocker, Michael (1990): Plural and Conflicting Values, Oxford: Clarendon Press

Stocker, Michael (1997): 'Abstract and Concrete Value: Plurality, Conflict, and Maximization', in: Incommensurability, Incomparability, and Practical Reason, R. Chang (Hg.), Cambridge: Harvard University Press Sunstein, Cass (1997): 'Incommensurability and Kinds of Valuation: Some Applications in Law', in: Incommensurability, Incomparability, and Practical Reason, R. Chang (Hg.), Cambridge: Harvard University Press Suzuki, Makoto (2011): 'Coping with Apparently Incomparable Alternatives - Pluralism, Parity and Justified Choice’, in: Dialogue and Universalism 2, 55-74

T Tennenbaum, Sergio (2015): 'Acting and Satisficing', in: Reasons and Intentions in Law and Practical Agency, G. Pavlakos und V. RodriguezBlanco (Hg.), Cambridge: Cambridge University Press

Tennenbaum, Sergio und Rafman, Diana (2012): 'Vague Projects and the Puzzle of the Self-Torturer', in: Ethics 123, 86-112 
Tugendhat, Ernst (1979): Selbstbewusstsein und Selbstbestimmung, Frankfurt am Main: Suhrkamp

Tugendhat, Ernst (2003): Egozentrizität und Mystik - eine anthropologische Studie, München: Verlag C. H. Beck

Tversky, Amos (1969): 'Intransitivity of preferences', in: Psychological Review 76, 31-48

U Ulmann-Margalit, Edna und Morgenbesser, Sidney (1977): 'Picking and Choosing', in: Social Research 44, 757-785

V Van Fraassen, Baastian (1966): 'Singular Terms, Truth-value Gaps and Free Logic', in: Journal of Philosophy 63, 481-495

Verbeek, Bruno (2008): 'Rational Self-Commitment', in: Rationality and Commitment, F. Peter und H. Schmid (Hg.), Oxford: Oxford University Press

- Von Neumann, John und Morgenstern, Oskar (1944): Theory of games and economic behavior, Princeton: Princeton University Press

W Warren, Caleb, McGraw, Peter und Van Boven, Leaf (2011): 'Values and preferences: defining preference construction', in: Wiley Interdisciplinary Reviews: Cognitive Science, 193-205

Waschkies, Hans-Joachim (1989): Anfänge der Arithmetik im Alten Orient und bei den Griechen, Amsterdam: B. R. Grüner

Wasserman, Ryan (2004): 'Indeterminacy, Ignorance and the Possibility of Parity' in: Philosophical Perspectives 18, 391-403

Weber, Michael (2000): 'A New Defense of Satisficing', in: Satisficing and Maximizing, M. Byron (Hg.), Cambridge: Cambridge University Press Wilson, Timothy (2002): Strangers to Ourselves: Discovering the adaptive Unconscious, Cambridge: Harvard University Press

Wittgenstein, Ludwig (1984): Philosophische Untersuchungen, Frankfurt am Main: Suhrkamp

Wolf, Jean-Claude (2005): 'Wertkonflikte', in: Wertkonflikte und Wertewandel, C. Giordano, J.-L. Patry (Hg.), Münster: Lit Verlag

Y Yitzhak, Benbaji (2009): 'Parity, Intransitivity, and a Context-Sensitive Degree Analysis of Gradability', in: Australasian Journal of Philosophy $87,313-335$ 


\section{Personenregister}

A Adler, M. 23, 34

Aldred, J. 27

Anderson, E. 31, 32, 34

Andreou, C. 20, 25, 92, 99,

111, 178, 193, 196

Anscombe, G. E. M. 52

Aristoteles 61, 212

Arpaly, N. 139

Audi, R. 139

Aumann, R. 158

B Badhwar, N. 223

Beisbart, C. 142, 143

Berlin, I. 32

Betzler, M. 32, 82, 241, 242

Boot, M. 13, 14, 23, 29, 103,

129, 162, 164, 166, 167

Bratman, M. 52

Breyer, F. 114

Broome, J. 14, 26, 33, 69, 147,

240

Brun, G. 42

Buchanan, A. 164

Byron, M. 134-136

C Carlson, E. 12, 26, 111, 119,

163,180

Carnap, R. 43

Chang, R. 12, 13, 19, 20, 27, 29, $32,33,35,37,46,55,63,72,78$,

79, 82, 89-91, 102, 103, 108,

111, 117, 123, 128-132, 134, 137,

$152,156-161,163,164,166,167$,

170-172, 174-176, 178-181,

183-189, 192, 193, 204, 211-

214, 216, 223, 237-241

Constantinescu, C. 25, 69, 166

Cubitt, R. 118
D D’Agostino, F. 24, 30, 31, 34

Daly, C. 40,72

Davidson, D. 59

De Jonge, J. 53, 150, 151

De Sousa, R. 104, 117

Dworkin, R. 31, 34

E Elson, L. 162, 163, 166

Espinoza, N. 12, 26, 109,

111, 115, 118-120

Elster, J. 103

F Feyerabend, P. 24

Fröding, B. 23

G Gert, J. 33, 111, 181-189

Glock, H.J. 42

Green, J. 158

Greimann, D. 43

Griffin, J. 25, 54, 74, 111,

156, 192

Grimm, S. 29

Guckes, B. 237

Gustafsson, J. 12, 26, 109 ,

111, 115, 117-120

H Hahn, S. 139

Hare, C. 119, 120

Hare, R. 100

Hausman, D. 50, 54, 56,

100, 139

Henning, T. 219, 220

Hirsch Hadorn, G. 42, 43

Hsieh, N. 14, 46, 108, 111,

137, 178, 192-211

Hume, D. 60

K Kavka, G. 25

Keefe, R. 69, 165

Klocksiem, J. 111

Kierkegaard, S. 146 
Kornhauser, L. 26, 27

Kuhn, T. 24

L Levi, I. 75, 151

Lichtenstein, S. 52, 56

Lindman, H. 52

Lockhart, T. 84

Lukes, S. 25, 31, 32

M MacAskill, W. 84

Mas-Colell, A. 13

McGraw, P. 56, 57, 98

Millgram, E. 222-224

Morgenbesser, S. 218

Morgenstern, O. 53

Morton, A. 26

N Nagel, T. 79

Nida-Rümelin, J. 141, 143

P Parfit, D. 111, 156, 192

Pauer-Studer, H. 238

Paul, L.A. 85, 86, 233

Peter, F. 238

Peterson, M. 23, 193

Pfister, J. 37

Pinkowski, D. 193, 204, 216

Platon, 36, 37

R Rabinowicz, W. 12, 25, 26,

111, 178, 181, 183-186

Rauschmayer, F. 124

Raz, J. 14, 25, 31-33, 63, 105,

108-113, 123, 143, 200, 237

Regan, D. 18, 123

Resnik, M. 13, 74, 75, 97, 98, 115

Reuter, K. 86, 103, 121, 123,

139, 217

Richardson, H. 34, 59, 60

Robinson, R. 41, 45

Rott, H. 136

S Sartre, J.-P. 74

Savage, L. 53, 115
Scanlon, T. 52, 143

Schaber, P. 12, 32, 35, 54,

62, 63, 90, 91, 216, 217

Schauer, F. 62

Schick, F. 139

Schmidtz, D. 136

Seel, G. 65

Sellmaier, S. 100

Sen, A. 100, 137, 138

Sepielli, S. 84

Shafir, E. 57

Simon, H. 135

Simonson, I. 57

Sinnott-Armstrong, W. 12, 23, 32, 34, 111

Slovic, P. 52, 56

Steglich-Petersen, A. 193, 194

Stocker, M. 27, 80

Sugden, R. 118

Sunstein, C. 23

Suzuki, M. 111, 129

T Tennenbaum, S. 232

Tugendhat, E. 65, 140, 149

Tversky, A. 57, 120, 121

U Ulmann-Margalit, E. 218

V Van Boven, L. 56, 57, 98

Van Fraassen, B. 69

V Verbeek, B. 240

Von Neumann, J. 53

W Warren, C. 56, 57, 98

Waschkies, H.J. 23

Weber, M. 135, 136

Whinston, M. 158

Wilson, T. 219

Wingert, L. 234-236

Wittgenstein, L. 39, 40

Wolf, J.-C. 81, 82, 147

Y Yitzhak, B. 178 


\section{Sachregister}

A Argument der kleinen Verbesserung 104-105, 107125, 160, 175, 184, 200

Autonomie (und

Würfeln) 241

Axiomatik der Entschei-

dungstheorie 97-99,159

B Bekenntnis 237-242

Begriffsanalyse 26-41

Beliebige Wahl (Konzept) 218-222

D Deckwert (Changs

Deutung) 211-214

Deckwert (Hsiehs

Deutung) 192-200

Definitionen von Un-

vergleichbarkeit und In-

kommensurabilität 22-35

Dynamischer Entschei-

dungskontext 229-231

E Evaluative Unsicher-

heit $83-87,225$

Empirische Evidenz 103,

121, 123, 217

Explikation 41-45

F Faktische Unsicherheit 75-

78, 226-227

G Gleichheit (gleich gut) 121-

124, 204, 217

Grenzen der Vergleich-

barkeit 145-149

H Harte Fälle 72-73, 78-93

I (In)Transitivität 108-109, 120-124, 150
K Ketten-Argument 160-176

Kohärenz 91, 132-134, 139,

231

P Parität (Changs

Deutung) 179-180

Parität (Gerts

Deutung) 180-184

Präferenzen 51-62

R Rationalität und (Un)Vergleichbarkeit 127-145

$\mathrm{T}$ Theorie aufgedeckter Präferenzen 30, 57-58, 110 Trichotomie-These 26, 29, 33, 35, 156-160

V Vages Entscheidungskriterium 171-176 Vagheit 38, 68-69, 120, 165-166, 232

W Wertkonflikt 78-83

Wertpluralismus 78-81

Wichtigkeit der Entscheidung 87-88

Wohlergehen 223-225, 227, 233-235 


\section{$\mathrm{Zu}$ Autor und Buch}

Handlungsentscheidungen sind ein Bestandteil unseres Lebens. Wir fällen sie täglich, oft unbewusst und routiniert. Diese Routine wird durchbrochen, wenn andere unsere Entscheidungen in Frage stellen oder wenn wir vor besonders gewichtigen Entscheidungen stehen, wie etwa der Berufswahl. In solchen Fällen versuchen wir die zur Wahl stehenden Optionen zu vergleichen. Dass wir das tun, sogar sagen können, welche Wahl die bessere oder schlechtere ist, scheint eine notwendige Bedingung für eine rationale Entscheidung zu sein. Die seit ca. dreissig Jahren etablierte Diskussion um Vergleichbarkeit und Inkommensurabilität von Werten beschäftigt sich mit sogenannten 'harten Fällen', in denen kein Vergleich möglich ist. Wer vor der Wahl steht, Anwältin oder Klarinettistin zu werden, hat gegebenenfalls grosse Schwierigkeiten, einen Beruf als besser oder schlechter als oder gleich gut wie den anderen auszuweisen. Doch wie lassen sich Unvergleichbarkeit und 'harte Fälle' genau charakterisieren, in welche Beziehung müssen Optionen gebracht werden, um Rationalität zu garantieren, und wie können wir mit solchen Konflikten umgehen? Das vorliegende Buch liefert neue Antworten auf diese Fragen.

Beleuchtet werden der Zusammenhang von Vergleichbarkeit und Rationalität, Transitivität, vage Entscheidungskriterien oder Werte. So wird dafür plädiert, dass bislang von einer irreführenden Auffassung von Werten ausgegangen wurde, die diese quasi-objektivistisch als etwas 'in der Welt' konzeptualisiert. Dabei wird verkannt, dass es gerade bei harten Fällen darum geht, dass wir selbst in ein wertendes Verhältnis zu dem gelangen, wofür wir uns zu entscheiden gedenken. Manchmal sind wir mit unvergleichbaren Optionen konfrontiert, können dabei oft nicht rational entscheiden und dürfen eine Münze werfen.

Michael Messerli hat an der Universität Bern Philosophie und Volkswirtschaftslehre studiert und 2017 an der LMU München in diesen Fächern promoviert (VWL jeweils im Nebenfach). Danach folgte ein Postdoc am philosophischen Institut der Universität Sheffield (UK) und eine Adam-Smith-Gastprofessur in Bayreuth. Zurzeit leitet er ein Ambizione-Projekt des Schweizerischen Nationalfonds am Ethik-Zentrum der Universität Zürich ('Transformative Experiences'). Seine Forschung handelt u.a. davon, wie wir Entscheidungen treffen und wie wir sie treffen sollen. 
'You want

to flip a coin

to see the

leads, Harvey?’ 